\title{
2,2-Bifunctionalization of Norbornene in Palladium-Catalyzed Domino Annulation
}

\author{
Si-Yi Yang, Wen-Yong Han, * Chen He, Bao-Dong Cui, Nan-Wei Wan, and \\ Yong-Zheng Chen* \\ Key Laboratory of Biocatalysis \& Chiral Drug Synthesis of Guizhou Province, Generic Drug \\ Research Center of Guizhou Province, School of Pharmacy, Zunyi Medical University, Zunyi \\ 563006, P. R. China \\ E-mail: hanwy@zmu.edu.cn; yzchen@zmu.edu.cn
}

\section{Supporting Information}

\section{Table of Contents}

1. General experimental information

2. Optimization of the reaction conditions for the construction of $\mathbf{4 a}$........................S1

3. Representative procedure for the synthesis of compound 4 a. ..............................S3

4. Characterization data of compounds 4a-ae, and 5-7 ............................................ 3

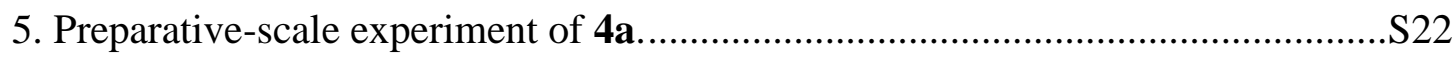

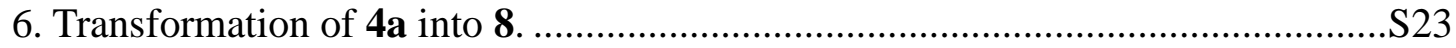

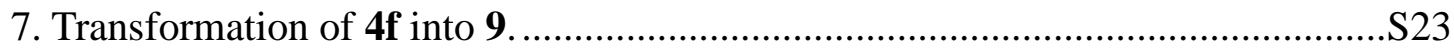

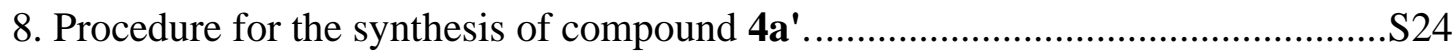

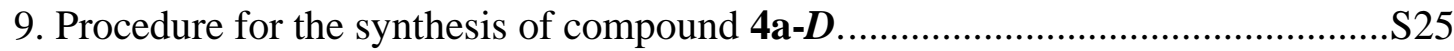

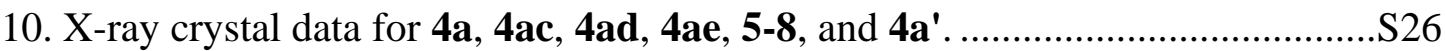

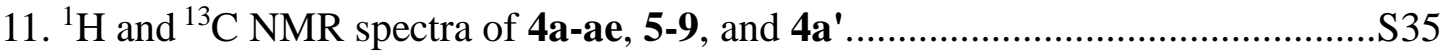

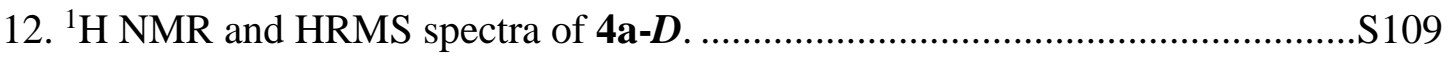




\section{General experimental information}

Unless otherwise noted, all commercially available reagents were used without further purification. All of the solvents were treated according to known methods. Column chromatography was performed on silica gel (200-400 mesh). ${ }^{1} \mathrm{H}$ NMR (400 MHz) chemical shifts were reported in ppm $(\delta)$ relative to tetramethylsilane (TMS) with the solvent resonance employed as the internal standard. ${ }^{13} \mathrm{C}$ NMR $(100 \mathrm{MHz})$ chemical shifts were reported in ppm $(\delta)$ from tetramethylsilane (TMS) with the solvent resonance as the internal standard. Data were reported as follows: chemical shift, multiplicity $(\mathrm{s}=$ singlet, $\mathrm{d}=$ doublet, $\mathrm{t}=$ triplet, $\mathrm{q}=$ quartet, $\mathrm{dd}=$ doublet of doublets, $\mathrm{td}=$ triplet of doublets, $\mathrm{m}=$ multiplet $)$, coupling constants $(\mathrm{Hz})$ and integration . HRMS measurements were obtained on a TOF analyzer. Melting points were uncorrected.

3-Iodochromones (1) was prepared according to the reported procedures. ${ }^{1}$ a-Bromoacetophenones (2), norbornene (NBE, 3a), and 1,4-dihydro-1,4-epoxynaphthalene (3b) were purchased from commercial suppliers. 1,4-Dihydro-1,4-methanonaphthalene (3c $)^{2}$ and 1,4-dihydro-1,4-ethanonaphthalene $(\mathbf{3 d})^{3}$ were prepared according to the reported procedures.

1. Vasselin, D. A.; Westwell, A. D.; Matthews, C. S.; Bradshaw, T. D.; Stevens, M. F. G. J. Med. Chem. 2006, 49, 3973-3981.

2. Medina, J. M.; Ko, J. H.; Maynard, H. D.; Garg, N. K. Macromolecules 2017, 50, 580-586.

3. Ishii, Y.; Ito, S.; Saito, Y.; Uno, D.; Oba, T. Tetrahedron 2015, 71, 8892-8898.

\section{Optimization of the reaction conditions for the construction of $4 a^{a}$}

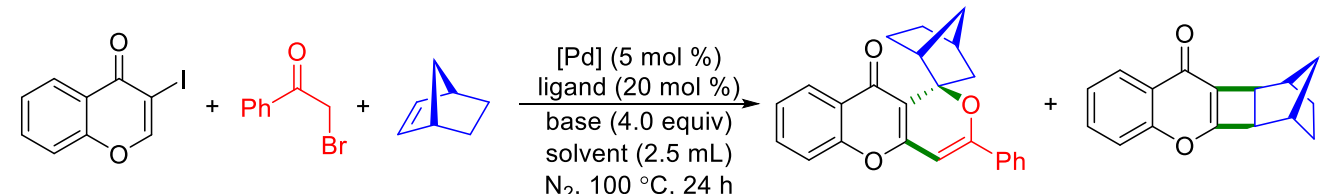

$1 \mathrm{a}$

$2 a$

$3 a$

$4 a$

$4 a^{\prime}$

\begin{tabular}{|c|c|c|c|c|c|c|c|}
\hline \multirow{2}{*}{ entry } & \multirow{2}{*}[\mathrm{Pd}]{} & \multirow{2}{*}{ ligand } & \multirow{2}{*}{ solvent } & \multirow{2}{*}{ base } & \multicolumn{2}{|c|}{ yield $(\%)^{b}$} & \multirow{2}{*}{$\mathrm{dr}^{c}$} \\
\hline & & & & & $4 a$ & $4 a^{\prime}$ & \\
\hline 1 & $\mathrm{Pd}\left(\mathrm{PPh}_{3}\right)_{4}$ & $\mathrm{PPh}_{3}$ & $\mathrm{PhMe}$ & $\mathrm{Cs}_{2} \mathrm{CO}_{3}$ & 18 & - & $83: 17$ \\
\hline 2 & $\mathrm{Pd}_{2}(\mathrm{dba})_{3}$ & $\mathrm{PPh}_{3}$ & $\mathrm{PhMe}$ & $\mathrm{Cs}_{2} \mathrm{CO}_{3}$ & 35 & - & $85: 14$ \\
\hline 3 & $\operatorname{Pd}(\mathrm{TFA})_{2}$ & $\mathrm{PPh}_{3}$ & $\mathrm{PhMe}$ & $\mathrm{Cs}_{2} \mathrm{CO}_{3}$ & 34 & - & $84: 16$ \\
\hline 4 & $\mathrm{Pd}(\mathrm{OAc})_{2}$ & $\mathrm{PPh}_{3}$ & $\mathrm{PhMe}$ & $\mathrm{Cs}_{2} \mathrm{CO}_{3}$ & 47 & - & $87: 13$ \\
\hline 5 & $\mathrm{Pd}(\mathrm{OPiv})_{2}$ & $\mathrm{PPh}_{3}$ & $\mathrm{PhMe}$ & $\mathrm{Cs}_{2} \mathrm{CO}_{3}$ & 55 & - & $86: 14$ \\
\hline 6 & {$[(\pi \text {-cinnamyl }) \mathrm{PdCl}]_{2}$} & $\mathrm{PPh}_{3}$ & $\mathrm{PhMe}$ & $\mathrm{Cs}_{2} \mathrm{CO}_{3}$ & 41 & - & $86: 14$ \\
\hline 7 & $\mathrm{Pd}(\mathrm{OPiv})_{2}$ & TFP & $\mathrm{PhMe}$ & $\mathrm{Cs}_{2} \mathrm{CO}_{3}$ & 52 & - & $85: 15$ \\
\hline
\end{tabular}




\begin{tabular}{|c|c|c|c|c|c|c|c|}
\hline 8 & $\mathrm{Pd}(\mathrm{OPiv})_{2}$ & $\mathrm{P}\left(4-\mathrm{CH}_{3}-\mathrm{C}_{6} \mathrm{H}_{4}\right)_{3}$ & $\mathrm{PhMe}$ & $\mathrm{Cs}_{2} \mathrm{CO}_{3}$ & 47 & - & $86: 14$ \\
\hline 9 & $\mathrm{Pd}(\mathrm{OPiv})_{2}$ & $\mathrm{P}\left(4-\mathrm{CF}_{3}-\mathrm{C}_{6} \mathrm{H}_{4}\right)_{3}$ & $\mathrm{PhMe}$ & $\mathrm{Cs}_{2} \mathrm{CO}_{3}$ & 58 & - & $85: 15$ \\
\hline 10 & $\mathrm{Pd}(\mathrm{OPiv})_{2}$ & $\mathrm{P}\left(4-\mathrm{F}-\mathrm{C}_{6} \mathrm{H}_{4}\right)_{3}$ & $\mathrm{PhMe}$ & $\mathrm{Cs}_{2} \mathrm{CO}_{3}$ & 60 & - & $86: 14$ \\
\hline 11 & $\mathrm{Pd}(\mathrm{OPiv})_{2}$ & $\mathrm{MePPh}_{2}$ & $\mathrm{PhMe}$ & $\mathrm{Cs}_{2} \mathrm{CO}_{3}$ & 20 & - & $85: 15$ \\
\hline 12 & $\mathrm{Pd}(\mathrm{OPiv})_{2}$ & 2-DPP-BP & $\mathrm{PhMe}$ & $\mathrm{Cs}_{2} \mathrm{CO}_{3}$ & 33 & - & $86: 14$ \\
\hline 13 & $\mathrm{Pd}(\mathrm{OPiv})_{2}$ & Xantphos & $\mathrm{PhMe}$ & $\mathrm{Cs}_{2} \mathrm{CO}_{3}$ & 8 & 15 & - \\
\hline 14 & $\mathrm{Pd}(\mathrm{OPiv})_{2}$ & XPhos & $\mathrm{PhMe}$ & $\mathrm{Cs}_{2} \mathrm{CO}_{3}$ & 5 & 12 & - \\
\hline 15 & $\mathrm{Pd}(\mathrm{OPiv})_{2}$ & $r a c$-BINAP & $\mathrm{PhMe}$ & $\mathrm{Cs}_{2} \mathrm{CO}_{3}$ & 7 & 17 & - \\
\hline 16 & $\mathrm{Pd}(\mathrm{OPiv})_{2}$ & $\mathrm{P}\left(4-\mathrm{F}-\mathrm{C}_{6} \mathrm{H}_{4}\right)_{3}$ & $o$-xylene & $\mathrm{Cs}_{2} \mathrm{CO}_{3}$ & 57 & - & $83: 17$ \\
\hline 17 & $\mathrm{Pd}(\mathrm{OPiv})_{2}$ & $\mathrm{P}\left(4-\mathrm{F}-\mathrm{C}_{6} \mathrm{H}_{4}\right)_{3}$ & $m$-xylene & $\mathrm{Cs}_{2} \mathrm{CO}_{3}$ & 49 & - & $85: 15$ \\
\hline 18 & $\mathrm{Pd}(\mathrm{OPiv})_{2}$ & $\mathrm{P}\left(4-\mathrm{F}-\mathrm{C}_{6} \mathrm{H}_{4}\right)_{3}$ & $p$-xylene & $\mathrm{Cs}_{2} \mathrm{CO}_{3}$ & 64 & - & $81: 19$ \\
\hline 19 & $\mathrm{Pd}(\mathrm{OPiv})_{2}$ & $\mathrm{P}\left(4-\mathrm{F}-\mathrm{C}_{6} \mathrm{H}_{4}\right)_{3}$ & mesitylene & $\mathrm{Cs}_{2} \mathrm{CO}_{3}$ & 67 & - & $86: 14$ \\
\hline 20 & $\mathrm{Pd}(\mathrm{OPiv})_{2}$ & $\mathrm{P}\left(4-\mathrm{F}-\mathrm{C}_{6} \mathrm{H}_{4}\right)_{3}$ & $\mathrm{PhCF}_{3}$ & $\mathrm{Cs}_{2} \mathrm{CO}_{3}$ & 21 & - & $88: 12$ \\
\hline 21 & $\mathrm{Pd}(\mathrm{OPiv})_{2}$ & $\mathrm{P}\left(4-\mathrm{F}-\mathrm{C}_{6} \mathrm{H}_{4}\right)_{3}$ & 1,4-dioxane & $\mathrm{Cs}_{2} \mathrm{CO}_{3}$ & 39 & - & $89: 11$ \\
\hline 22 & $\mathrm{Pd}(\mathrm{OPiv})_{2}$ & $\mathrm{P}\left(4-\mathrm{F}-\mathrm{C}_{6} \mathrm{H}_{4}\right)_{3}$ & DCE & $\mathrm{Cs}_{2} \mathrm{CO}_{3}$ & 43 & - & $83: 17$ \\
\hline 23 & $\mathrm{Pd}(\mathrm{OPiv})_{2}$ & $\mathrm{P}\left(4-\mathrm{F}-\mathrm{C}_{6} \mathrm{H}_{4}\right)_{3}$ & Glyme & $\mathrm{Cs}_{2} \mathrm{CO}_{3}$ & 25 & - & $86: 14$ \\
\hline 24 & $\mathrm{Pd}(\mathrm{OPiv})_{2}$ & $\mathrm{P}\left(4-\mathrm{F}-\mathrm{C}_{6} \mathrm{H}_{4}\right)_{3}$ & DMSO & $\mathrm{Cs}_{2} \mathrm{CO}_{3}$ & trace & - & - \\
\hline 25 & $\mathrm{Pd}(\mathrm{OPiv})_{2}$ & $\mathrm{P}\left(4-\mathrm{F}-\mathrm{C}_{6} \mathrm{H}_{4}\right)_{3}$ & DMF & $\mathrm{Cs}_{2} \mathrm{CO}_{3}$ & trace & - & - \\
\hline 26 & $\mathrm{Pd}(\mathrm{OPiv})_{2}$ & $\mathrm{P}\left(4-\mathrm{F}-\mathrm{C}_{6} \mathrm{H}_{4}\right)_{3}$ & mesitylene & $\mathrm{Na}_{2} \mathrm{CO}_{3}$ & 41 & - & $85: 15$ \\
\hline 27 & $\mathrm{Pd}(\mathrm{OPiv})_{2}$ & $\mathrm{P}\left(4-\mathrm{F}-\mathrm{C}_{6} \mathrm{H}_{4}\right)_{3}$ & mesitylene & $\mathrm{K}_{2} \mathrm{CO}_{3}$ & 56 & - & $85: 15$ \\
\hline 28 & $\mathrm{Pd}(\mathrm{OPiv})_{2}$ & $\mathrm{P}\left(4-\mathrm{F}-\mathrm{C}_{6} \mathrm{H}_{4}\right)_{3}$ & mesitylene & $\mathrm{K}_{3} \mathrm{PO}_{4}$ & 70 & - & $86: 14$ \\
\hline 29 & $\mathrm{Pd}(\mathrm{OPiv})_{2}$ & $\mathrm{P}\left(4-\mathrm{F}-\mathrm{C}_{6} \mathrm{H}_{4}\right)_{3}$ & mesitylene & $\mathrm{NaOAc}$ & 29 & - & $85: 15$ \\
\hline 30 & $\mathrm{Pd}(\mathrm{OPiv})_{2}$ & $\mathrm{P}\left(4-\mathrm{F}-\mathrm{C}_{6} \mathrm{H}_{4}\right)_{3}$ & mesitylene & $\mathrm{NaOPiv}$ & 41 & - & $84: 16$ \\
\hline 31 & $\mathrm{Pd}(\mathrm{OPiv})_{2}$ & $\mathrm{P}\left(4-\mathrm{F}-\mathrm{C}_{6} \mathrm{H}_{4}\right)_{3}$ & mesitylene & KOAc & 47 & - & $83: 17$ \\
\hline $32^{d}$ & $\mathrm{Pd}(\mathrm{OPiv})_{2}$ & $\mathrm{P}\left(4-\mathrm{F}-\mathrm{C}_{6} \mathrm{H}_{4}\right)_{3}$ & mesitylene & $\mathrm{K}_{3} \mathrm{PO}_{4}$ & 63 & - & $85: 15$ \\
\hline $33^{e}$ & $\mathrm{Pd}(\mathrm{OPiv})_{2}$ & $\mathrm{P}\left(4-\mathrm{F}-\mathrm{C}_{6} \mathrm{H}_{4}\right)_{3}$ & mesitylene & $\mathrm{K}_{3} \mathrm{PO}_{4}$ & 66 & - & $86: 14$ \\
\hline $34^{f}$ & $\mathrm{Pd}(\mathrm{OPiv})_{2}$ & $\mathrm{P}\left(4-\mathrm{F}-\mathrm{C}_{6} \mathrm{H}_{4}\right)_{3}$ & mesitylene & $\mathrm{K}_{3} \mathrm{PO}_{4}$ & 53 & - & $85: 15$ \\
\hline $35^{g}$ & $\mathrm{Pd}(\mathrm{OPiv})_{2}$ & $\mathrm{P}\left(4-\mathrm{F}-\mathrm{C}_{6} \mathrm{H}_{4}\right)_{3}$ & mesitylene & $\mathrm{K}_{3} \mathrm{PO}_{4}$ & 79 & - & $86: 14$ \\
\hline $36^{g, h}$ & $\mathrm{Pd}(\mathrm{OPiv})_{2}$ & $\mathrm{P}\left(4-\mathrm{F}-\mathrm{C}_{6} \mathrm{H}_{4}\right)_{3}$ & mesitylene & $\mathrm{K}_{3} \mathrm{PO}_{4}$ & 63 & - & $85: 15$ \\
\hline $37^{g, i}$ & $\mathrm{Pd}(\mathrm{OPiv})_{2}$ & $\mathrm{P}\left(4-\mathrm{F}-\mathrm{C}_{6} \mathrm{H}_{4}\right)_{3}$ & mesitylene & $\mathrm{K}_{3} \mathrm{PO}_{4}$ & 62 & - & $86: 14$ \\
\hline $38^{g, j}$ & $\mathrm{Pd}(\mathrm{OPiv})_{2}$ & $\mathrm{P}\left(4-\mathrm{F}-\mathrm{C}_{6} \mathrm{H}_{4}\right)_{3}$ & mesitylene & $\mathrm{K}_{3} \mathrm{PO}_{4}$ & 55 & - & $86: 14$ \\
\hline $39^{g, k}$ & $\mathrm{Pd}(\mathrm{OPiv})_{2}$ & $\mathrm{P}\left(4-\mathrm{F}-\mathrm{C}_{6} \mathrm{H}_{4}\right)_{3}$ & mesitylene & $\mathrm{K}_{3} \mathrm{PO}_{4}$ & 83 & - & $88: 12$ \\
\hline $40^{g, l}$ & $\mathrm{Pd}(\mathrm{OPiv})_{2}$ & $\mathrm{P}\left(4-\mathrm{F}-\mathrm{C}_{6} \mathrm{H}_{4}\right)_{3}$ & mesitylene & $\mathrm{K}_{3} \mathrm{PO}_{4}$ & 81 & - & $86: 14$ \\
\hline $41^{g, k, m}$ & $\mathrm{Pd}(\mathrm{OPiv})_{2}$ & $\mathrm{P}\left(4-\mathrm{F}-\mathrm{C}_{6} \mathrm{H}_{4}\right)_{3}$ & mesitylene & $\mathrm{K}_{3} \mathrm{PO}_{4}$ & 77 & - & $86: 14$ \\
\hline $42^{g, k, n}$ & $\mathrm{Pd}(\mathrm{OPiv})_{2}$ & $\mathrm{P}\left(4-\mathrm{F}-\mathrm{C}_{6} \mathrm{H}_{4}\right)_{3}$ & mesitylene & $\mathrm{K}_{3} \mathrm{PO}_{4}$ & 78 & - & $87: 13$ \\
\hline
\end{tabular}

${ }^{a}$ Unless otherwise noted, all reactions were performed with 1a $(0.4 \mathrm{mmol}, 1.0$ equiv), 2a (0.4 mmol, 1.0 equiv), 3a (1.6 mmol, 4.0 equiv), Pd-catalyst ( $5 \mathrm{~mol} \%)$, ligand (20 mol \%), base (1.6 mmol, 4.0 equiv) in $2.5 \mathrm{~mL}$ of solvent under $\mathrm{N}_{2}$ atmosphere at $100{ }^{\circ} \mathrm{C}$ for $24 \mathrm{~h} .{ }^{b}$ Isolated yield based on 1a. ${ }^{c}$ Determined by ${ }^{1} \mathrm{H}$ NMR analysis. ${ }^{d} 3.0$ equiv 3a was used. ${ }^{e} 5.0$ equiv 3a was used. ${ }^{f} 3 \mathrm{~mol} \% \mathrm{Pd}(\mathrm{OPiv})_{2}$ was used. ${ }^{g} 10 \mathrm{~mol} \% \mathrm{Pd}(\mathrm{OPiv})_{2}$ was used. ${ }^{h} 10 \mathrm{~mol} \% \mathrm{P}\left(4-\mathrm{F}-\mathrm{C}_{6} \mathrm{H}_{4}\right)_{3}$ was used. ${ }^{i} 30$ mol $\% \mathrm{P}\left(4-\mathrm{F}-\mathrm{C}_{6} \mathrm{H}_{4}\right)_{3}$ was used. ${ }^{j} 1.0$ equiv $\mathrm{K}_{3} \mathrm{PO}_{4}$ was used. ${ }^{k} 2.0$ equiv $\mathrm{K}_{3} \mathrm{PO}_{4}$ was used. 
${ }^{l} 3.0$ equiv $\mathrm{K}_{3} \mathrm{PO}_{4}$ was used. ${ }^{m}$ Run at $80{ }^{\circ} \mathrm{C}$. ${ }^{n}$ Run at $120{ }^{\circ} \mathrm{C}$. TFP $=\operatorname{tri}(2$-furyl)phosphine, 2-DPP-BP = 2-(diphenylphosphino)-biphenyl, Xantphos $=4,5$-bis(diphenyl-phosphino)-9,9dimethylxanthene, XPhos= 2-(dicyclohexylphosphino)-2,4,6-triisopropylbiphenyl, BINAP = 2,2'-bis(diphenyl phosphino)-1,1'-binaphthalene.

\section{Representative procedure for the synthesis of compound 4a (Scheme 2).}

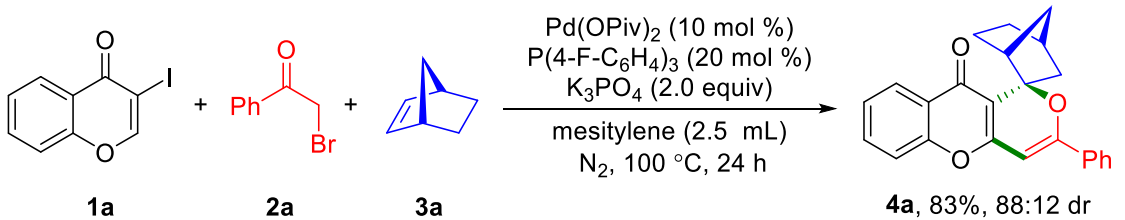

A $4 \mathrm{~mL}$ flame-dried vial with a stir bar was charged with 3-iodochromone (1a, $108.8 \mathrm{mg}, 0.4$ mmol), $\alpha$-bromoacetophenone (2a, $79.6 \mathrm{mg}, 0.4 \mathrm{mmol}$ ), NBE (3a, $150.6 \mathrm{mg}, 1.6 \mathrm{mmol}$ ), $\mathrm{Pd}(\mathrm{OPiv})_{2}(12.3 \mathrm{mg}, 0.04 \mathrm{mmol}), \mathrm{P}-\left(4-\mathrm{F}-\mathrm{C}_{6} \mathrm{H}_{4}\right)_{3}(25.3 \mathrm{mg}, 0.08 \mathrm{mmol})$, and $\mathrm{K}_{3} \mathrm{PO}_{4}(169.8 \mathrm{mg}, 0.8$ mmol) in $2.5 \mathrm{~mL}$ of dry mesitylene under nitrogen atmosphere at $100{ }^{\circ} \mathrm{C}$ for $24 \mathrm{~h}$. After the completion of the reaction detected by thin layer chromatography (TLC), the mixture was cooled to room temperature and purified by flash column chromatography on silica gel (petroleum ether/ethyl acetate/dichloromethane $=20: 1: 0.1-3: 1: 0.1)$ to afford the desired product $4 \mathbf{a}$ as a yellow solid (118.5 mg, 83\%, 88:12 dr).

\section{Characterization data of compounds 4a-ae, and 5-7}

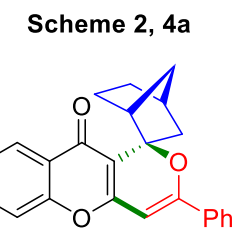

Compound 4a: purification by flash column chromatography on silica gel (eluent: petroleum ether/ethyl acetate/dichloromethane $=20: 1: 0.1-3: 1: 0.1)$; yellow solid, $118.5 \mathrm{mg}, 83 \%$ yield, $\mathrm{mp}$ $163.2-165.0{ }^{\circ} \mathrm{C} ; 88: 12 \mathrm{dr} ;{ }^{1} \mathrm{H}$ NMR $\left(400 \mathrm{MHz}, \mathrm{CDCl}_{3}\right) \delta$ (major + minor) $8.20(\mathrm{~d}, J=8.0 \mathrm{~Hz}$, $0.88 \mathrm{H}), 7.96(\mathrm{~d}, J=7.8 \mathrm{~Hz}, 0.12 \mathrm{H}), 7.91(\mathrm{~d}, J=7.8 \mathrm{~Hz}, 0.12 \mathrm{H}), 7.85-7.83(\mathrm{~m}, 0.24 \mathrm{H}), 7.80-$ $7.79(\mathrm{~m}, 1.76 \mathrm{H}), 7.59$ (t, $J=7.8 \mathrm{~Hz}, 0.88 \mathrm{H}), 7.47-7.43(\mathrm{~m}, 3 \mathrm{H}), 7.39-7.32(\mathrm{~m}, 2 \mathrm{H}), 6.35(\mathrm{~s}$, $0.12 \mathrm{H}), 6.29(\mathrm{~s}, 0.88 \mathrm{H}), 3.92(\mathrm{ddd}, J=13.4,5.0,2.8 \mathrm{~Hz}, 0.12 \mathrm{H}), 3.82(\mathrm{dd}, J=13.4,2.5 \mathrm{~Hz}$, $0.88 \mathrm{H}), 2.86(\mathrm{~s}, 0.88 \mathrm{H}), 2.78(\mathrm{~s}, 0.12 \mathrm{H}), 2.61(\mathrm{~s}, 0.12 \mathrm{H}), 2.51(\mathrm{~s}, 0.88 \mathrm{H}), 2.17(\mathrm{~d}, J=10.0 \mathrm{~Hz}$ $0.12 \mathrm{H}), 1.98(\mathrm{~d}, J=10.0 \mathrm{~Hz}, 0.88 \mathrm{H}), 1.86-1.78(\mathrm{~m}, 1.76 \mathrm{H}), 1.69-1.75(\mathrm{~m}, 0.12 \mathrm{H}), 1.59(\mathrm{dd}, J=$ 13.2, $3.6 \mathrm{~Hz}, 0.24 \mathrm{H}), 1.51-1.46(\mathrm{~m}, 0.88 \mathrm{H}), 1.27-1.18(\mathrm{~m}, 3 \mathrm{H}) ;{ }^{13} \mathrm{C} \mathrm{NMR}\left(100 \mathrm{MHz}, \mathrm{CDCl}_{3}\right) \delta$ 
(major + minor) 175.7, 162.2, 161.8, 154.8, 132.9, 132.8, 131.2, 131.1, 128.9, 128.8, 126.3, 126.1, 126.0, 125.1, 124.7, 117.4, 106.8, 95.0, 94.5, 90.7, 90.1, 48.5, 44.4, 41.1, 39.4, 37.8, 36.8, 36.6, 36.0, 29.8, 29.3, 28.0, 27.3, 23.2, 22.3. HRMS (ESI-TOF): calcd. for $\mathrm{C}_{24} \mathrm{H}_{21} \mathrm{O}_{3}[\mathrm{M}+\mathrm{H}]^{+} 357.1485$; found 357.1491 .

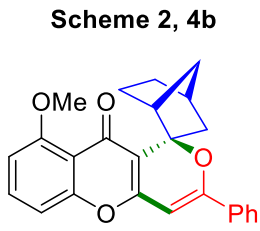

Compound 4b: purification by flash column chromatography on silica gel (eluent: petroleum ether/ethyl acetate/dichloromethane $=20: 1: 0.1-3: 1: 0.1) ;$ yellow solid, $80.4 \mathrm{mg}, 52 \%$ yield, $\mathrm{mp}$ $170.8-171.2{ }^{\circ} \mathrm{C} ; 90: 10 \mathrm{dr} ;{ }^{1} \mathrm{H}$ NMR $\left(400 \mathrm{MHz}, \mathrm{CDCl}_{3}\right) \delta$ (major + minor) $7.83-7.81(\mathrm{~m}, 0.20 \mathrm{H})$, $7.78-7.76(\mathrm{~m}, 1.80 \mathrm{H}), 7.48-7.47(\mathrm{~m}, 0.4 \mathrm{H}), 7.46-7.42(\mathrm{~m}, 3.60 \mathrm{H}), 6.94(\mathrm{dd}, J=8.4,0.92 \mathrm{~Hz}$, 1H), $6.75(\mathrm{~d}, J=8.0 \mathrm{~Hz}, 1 \mathrm{H}), 6.28(\mathrm{~s}, 0.10 \mathrm{H}), 6.23(\mathrm{~s}, 0.90 \mathrm{H}), 3.96(\mathrm{~s}, 3 \mathrm{H}), 3.77$ (dd, $J=13.4,2.0$ $\mathrm{Hz}, 1 \mathrm{H}), 2.86(\mathrm{~d}, J=4.4 \mathrm{~Hz}, 0.90 \mathrm{H}), 2.75(\mathrm{~d}, J=3.8 \mathrm{~Hz}, 0.10 \mathrm{H}), 2.48-2.44(\mathrm{~m}, 1 \mathrm{H}), 2.29(\mathrm{~d}, J=$ $10.8 \mathrm{~Hz}, 0.20 \mathrm{H}), 1.95-1.90(\mathrm{t}, J=11.4 \mathrm{~Hz}, 1.80 \mathrm{H}), 1.76(\mathrm{~d}, J=13.2 \mathrm{~Hz}, 1 \mathrm{H}), 1.51-1.42(\mathrm{~m}$, $1 \mathrm{H}), 1.29-1.24(\mathrm{~m}, 2 \mathrm{H}), 1.14(\mathrm{~d}, J=9.8 \mathrm{~Hz}, 1 \mathrm{H}) ;{ }^{13} \mathrm{C} \mathrm{NMR}\left(100 \mathrm{MHz}, \mathrm{CDCl}_{3}\right) \delta$ (major + minor) $176.0,161.5,160.1,159.9,156.9,133.0,132.8,131.0,130.8,128.8,128.7,126.2,125.8,115.6$, 109.7, 107.9, 106.2, 94.6, 94.2, 90.9, 56.6, 56.5, 48.3, 44.1, 40.8, 38.9, 37.9, 36.7, 36.6, 35.9, 29.3, 28.0, 23.5, 22.4. HRMS (ESI-TOF): calcd. for $\mathrm{C}_{25} \mathrm{H}_{23} \mathrm{O}_{4}[\mathrm{M}+\mathrm{H}]^{+}$387.1591; found 387.1597 .

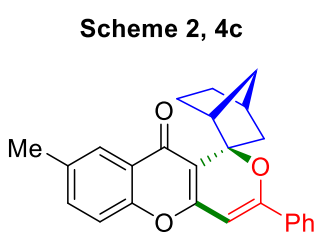

Compound 4c: purification by flash column chromatography on silica gel (eluent: petroleum ether/ethyl acetate $=20: 1-4: 1$ ); yellow solid, $110.4 \mathrm{mg}, 75 \%$ yield, mp $170.6-171.8{ }^{\circ} \mathrm{C} ; 88: 12$ $\mathrm{dr} ;{ }^{1} \mathrm{H} \mathrm{NMR}\left(400 \mathrm{MHz}, \mathrm{CDCl}_{3}\right) \delta$ (major + minor) $8.00(\mathrm{~s}, 1 \mathrm{H}), 7.84(\mathrm{dd}, J=6.4,2.8 \mathrm{~Hz}, 0.24 \mathrm{H})$, $7.81-7.77(\mathrm{~m}, 1.76 \mathrm{H}), 7.45-7.43(\mathrm{~m}, 3 \mathrm{H}), 7.39(\mathrm{dd}, J=8.4,1.8 \mathrm{~Hz}, 1 \mathrm{H}), 7.29-7.26(\mathrm{~m}, 1 \mathrm{H})$, $6.34(\mathrm{~s}, 0.12 \mathrm{H}), 6.28(\mathrm{~s}, 0.88 \mathrm{H}), 3.92(\mathrm{dd}, J=13.2,2.0 \mathrm{~Hz}, 0.12 \mathrm{H}), 3.83(\mathrm{dd}, J=13.4,2.0 \mathrm{~Hz}$, $0.88 \mathrm{H}), 2.85(\mathrm{~d}, J=4.0 \mathrm{~Hz}, 0.88 \mathrm{H}), 2.78(\mathrm{~d}, J=3.8 \mathrm{~Hz}, 0.12 \mathrm{H}), 2.51(\mathrm{~s}, 1 \mathrm{H}), 2.43(\mathrm{~s}, 3 \mathrm{H}), 2.17(\mathrm{~d}$, 
$J=10.0 \mathrm{~Hz}, 0.12 \mathrm{H}), 1.98(\mathrm{~d}, J=9.6 \mathrm{~Hz}, 0.88 \mathrm{H}), 1.85-1.79(\mathrm{~m}, 2 \mathrm{H}), 1.51-1.45(\mathrm{~m}, 1 \mathrm{H}), 1.29-$ $1.17(\mathrm{~m}, 3 \mathrm{H}) ;{ }^{13} \mathrm{C} \mathrm{NMR}\left(100 \mathrm{MHz}, \mathrm{CDCl}_{3}\right) \delta$ (major + minor) 175.8, 162.1, 161.7, 153.0, 134.5, 134.0, 133.9, 133.0, 132.1, 131.1, 131.0, 128.8, 128.7, 126.3, 125.9, 125.6, 124.7, 117.2, 106.7, 95.1, 94.6, 90.7, 48.5, 44.4, 41.1, 39.4, 37.7, 36.8, 36.6, 36.0, 29.3, 27.9, 23.2, 22.3, 21.1, 21.1. HRMS (ESI-TOF): calcd. for $\mathrm{C}_{25} \mathrm{H}_{23} \mathrm{O}_{3}[\mathrm{M}+\mathrm{H}]^{+}$371.1642; found 371.1646.

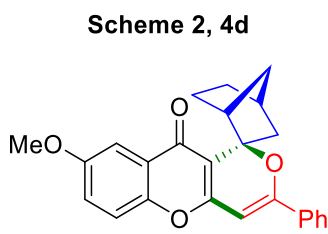

Compound 4d: purification by flash column chromatography on silica gel (eluent: petroleum ether/ethyl acetate $=20: 1-4: 1$ ); yellow solid, $105.1 \mathrm{mg}$, 68\% yield, mp $170.8-172.4{ }^{\circ} \mathrm{C} ; 90: 10$ $\mathrm{dr} ;{ }^{1} \mathrm{H}$ NMR $\left(400 \mathrm{MHz}, \mathrm{CDCl}_{3}\right) \delta$ (major + minor) $7.85-7.82(\mathrm{~m}, 0.20 \mathrm{H}), 7.81-7.77(\mathrm{~m}, 1.80 \mathrm{H})$, $7.60(\mathrm{~d}, J=2.8 \mathrm{~Hz}, 1 \mathrm{H}), 7.45-7.44(\mathrm{~m}, 3 \mathrm{H}), 7.31(\mathrm{~d}, J=9.0 \mathrm{~Hz}, 1 \mathrm{H}), 7.18(\mathrm{dd}, J=9.0,2.8 \mathrm{~Hz}$, 1H), $6.34(\mathrm{~s}, 0.10 \mathrm{H}), 6.28(\mathrm{~s}, 0.90 \mathrm{H}), 3.88(\mathrm{~s}, 3 \mathrm{H}), 3.82(\mathrm{~d}, J=13.4 \mathrm{~Hz}, 1 \mathrm{H}), 2.86(\mathrm{~d}, J=4.0 \mathrm{~Hz}$, $0.90 \mathrm{H}), 2.78(\mathrm{~d}, J=3.8 \mathrm{~Hz}, 0.10 \mathrm{H}), 2.52-2.50(\mathrm{~m}, 0.90 \mathrm{H}), 2.48-2.47(\mathrm{~m}, 0.10 \mathrm{H}), 2.18(\mathrm{~d}, J=$ $10.2 \mathrm{~Hz}, 0.10 \mathrm{H}), 1.98(\mathrm{~d}, J=9.8 \mathrm{~Hz}, 0.90 \mathrm{H}), 1.87-1.78(\mathrm{~m}, 2 \mathrm{H}), 1.54-1.45(\mathrm{~m}, 1 \mathrm{H}), 1.26-$ $1.18(\mathrm{~m}, 3 \mathrm{H}) ;{ }^{13} \mathrm{C}$ NMR $\left(100 \mathrm{MHz}, \mathrm{CDCl}_{3}\right) \delta$ (major + minor) 175.4, 162.0, 161.7, 156.7, 149.5, 132.9, 131.0, 128.8, 128.8, 126.3, 125.9, 125.7, 122.8, 122.6, 118.8, 106.3, 105.5, 95.0, 94.6, 90.8, 56.0, 48.5, 44.4, 41.1, 39.4, 37.7, 36.8, 36.6, 36.0, 29.4, 28.0, 23.2, 22.3. HRMS (ESI-TOF): calcd. for $\mathrm{C}_{25} \mathrm{H}_{23} \mathrm{O}_{4}[\mathrm{M}+\mathrm{H}]^{+}$387.1591; found 387.1597.

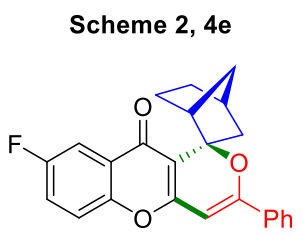

Compound 4e: purification by flash column chromatography on silica gel (eluent: petroleum ether/ethyl acetate $=20: 1-4: 1)$; yellow solid, $106.6 \mathrm{mg}, 71 \%$ yield, $\mathrm{mp} 181.6-183.4{ }^{\circ} \mathrm{C} ; 89: 11$ $\mathrm{dr} ;{ }^{1} \mathrm{H}$ NMR $\left(400 \mathrm{MHz}, \mathrm{CDCl}_{3}\right) \delta$ (major + minor) $7.86-7.78(\mathrm{~m}, 2.67 \mathrm{H}), 7.66-7.63(\mathrm{~m}, 0.33 \mathrm{H})$, $7.50-7.43(\mathrm{~m}, 2.67 \mathrm{H}), 7.40-7.37(\mathrm{~m}, 1 \mathrm{H}), 7.33-7.30(\mathrm{~m}, 1 \mathrm{H}), 7.20-7.16(\mathrm{~m}, 0.33 \mathrm{H}), 6.34(\mathrm{~s}$, $0.11 \mathrm{H}), 6.28(\mathrm{~s}, 0.89 \mathrm{H}), 3.90-3.85(\mathrm{~m}, 0.11 \mathrm{H}), 3.79(\mathrm{dd}, J=13.2,2.4 \mathrm{~Hz}, 0.89 \mathrm{H}), 2.86-2.85(\mathrm{~m}$, 
$0.89 \mathrm{H}), 2.78-2.77(\mathrm{~m}, 0.11 \mathrm{H}), 2.52-2.47(\mathrm{~m}, 1 \mathrm{H}), 2.13(\mathrm{~d}, J=10.2 \mathrm{~Hz}, 0.11 \mathrm{H}), 1.97(\mathrm{~d}, J=9.8$ $\mathrm{Hz}, 0.89 \mathrm{H}), 1.86-1.74(\mathrm{~m}, 1.78 \mathrm{H}), 1.62-1.58(\mathrm{~m}, 0.22 \mathrm{H}), 1.52-1.47(\mathrm{~m}, 1 \mathrm{H}), 1.30-1.18(\mathrm{~m}$, $2.67 \mathrm{H}), 0.88-0.85(\mathrm{~m}, 0.33 \mathrm{H}) ;{ }^{13} \mathrm{C} \mathrm{NMR}\left(100 \mathrm{MHz}, \mathrm{CDCl}_{3}\right) \delta$ (major + minor) $174.7,162.4(\mathrm{~d}, J$ $=52.7 \mathrm{~Hz}), 159.5(\mathrm{~d}, J=244.0 \mathrm{~Hz}), 151.0,132.8,131.2,128.9,128.8,126.4,126.0,120.8(\mathrm{~d}, J=$ $25.3 \mathrm{~Hz}), 119.4(\mathrm{~d}, J=8.0 \mathrm{~Hz}), 111.2(\mathrm{~d}, J=23.9 \mathrm{~Hz}), 106.2,94.3,90.8,48.5,44.4,41.1,39.4$, 37.8, 36.8, 36.6, 36.0, 29.3, 27.9, 27.0, 23.2, 22.3. HRMS (ESI-TOF): calcd. for $\mathrm{C}_{24} \mathrm{H}_{20} \mathrm{FO}_{3}[\mathrm{M}+$ $\mathrm{H}]^{+}$375.1391; found 375.1399 .

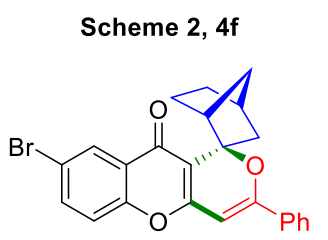

Compound 4f: purification by flash column chromatography on silica gel (eluent: petroleum ether/ethyl acetate/dichloromethane $=20: 1: 0.1-3: 1: 0.1)$; light yellow solid, $104.5 \mathrm{mg}, 60 \%$ yield, mp 159.5 - $161.0{ }^{\circ} \mathrm{C} ; 90: 10 \mathrm{dr} ;{ }^{1} \mathrm{H}$ NMR $\left(400 \mathrm{MHz}, \mathrm{CDCl}_{3}\right) \delta$ (major + minor) $8.31-8.28(\mathrm{~m}$, 1H), $7.82-7.77(\mathrm{~m}, 2 \mathrm{H}), 7.64(\mathrm{dd}, J=8.8,2.6 \mathrm{~Hz}, 1 \mathrm{H}), 7.46-7.42(\mathrm{~m}, 3 \mathrm{H}), 7.26-7.23(\mathrm{~m}, 1 \mathrm{H})$, $6.31(\mathrm{~s}, 0.10 \mathrm{H}), 6.25(\mathrm{~s}, 0.90 \mathrm{H}), 3.87-3.76(\mathrm{~m}, 1 \mathrm{H}), 2.85-2.84(\mathrm{~m}, 0.90 \mathrm{H}), 2.78-2.77(\mathrm{~m}$, $0.10 \mathrm{H}), 2.52-2.47(\mathrm{~m}, 1 \mathrm{H}), 2.12(\mathrm{~d}, J=10.2 \mathrm{~Hz}, 0.10 \mathrm{H}), 1.96(\mathrm{~d}, J=9.8 \mathrm{~Hz}, 0.90 \mathrm{H}), 1.86-1.77$ $(\mathrm{m}, 2 \mathrm{H}), 1.60-1.45(\mathrm{~m}, 1 \mathrm{H}), 1.29-1.18(\mathrm{~m}, 2.70 \mathrm{H}), 0.90-0.83(\mathrm{~m}, 0.30 \mathrm{H}) ;{ }^{13} \mathrm{C}$ NMR $(100$ $\left.\mathrm{MHz}, \mathrm{CDCl}_{3}\right) \delta$ (major + minor) 174.1, 162.8, 162.0, 153.5, 135.7, 135.6, 132.7, 131.4, 131.2, 128.9, 128.8, 128.7, 126.5, 126.4, 126.0, 119.4, 119.3, 118.1, 109.9, 106.7, 106.6, 94.7, 94.2, 90.7, 90.1, 48.4, 44.4, 41.1, 39.4, 37.8, 36.7, 36.5, 36.0, 29.2, 27.8, 23.2 22.2. HRMS (ESI-TOF): calcd. for $\mathrm{C}_{24} \mathrm{H}_{19} \mathrm{BrNaO}_{3}[\mathrm{M}+\mathrm{Na}]^{+} 457.0410$; found 457.0411 .

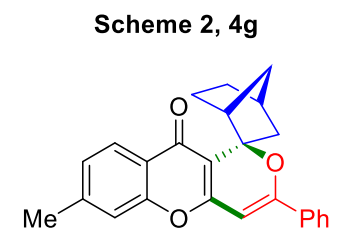

Compound 4g: purification by flash column chromatography on silica gel (eluent: petroleum ether/ethyl acetate $=20: 1-4: 1$; yellow solid, $118.5 \mathrm{mg}, 80 \%$ yield, mp $168.7-170.3{ }^{\circ} \mathrm{C} ; 90: 10$ $\mathrm{dr} ;{ }^{1} \mathrm{H}$ NMR $\left(400 \mathrm{MHz}, \mathrm{CDCl}_{3}\right) \delta$ (major + minor) $8.08(\mathrm{~d}, J=8.0 \mathrm{~Hz}, 1 \mathrm{H}), 7.85-7.83(\mathrm{~m}$, 
$0.20 \mathrm{H}), 7.80-7.78(\mathrm{~m}, 1.80 \mathrm{H}), 7.46-7.41(\mathrm{~m}, 3 \mathrm{H}), 7.17-7.14(\mathrm{~m}, 2 \mathrm{H}), 6.33(\mathrm{~s}, 0.10 \mathrm{H}), 6.27(\mathrm{~s}$, $0.90 \mathrm{H}), 3.95-3.90(\mathrm{~m}, 0.10 \mathrm{H}), 3.83(\mathrm{dd}, J=13.4,1.2 \mathrm{~Hz}, 0.90 \mathrm{H}), 2.86(\mathrm{~d}, J=4.0 \mathrm{~Hz}, 0.90 \mathrm{H})$, $2.77(\mathrm{~d}, J=3.8 \mathrm{~Hz}, 0.10 \mathrm{H}), 2.52-2.49(\mathrm{~m}, 1 \mathrm{H}), 2.46(\mathrm{~s}, 3 \mathrm{H}), 2.18(\mathrm{~d}, J=11.2 \mathrm{~Hz}, 0.10 \mathrm{H}), 1.97$ $(\mathrm{d}, J=9.6 \mathrm{~Hz}, 0.90 \mathrm{H}), 1.85-1.79(\mathrm{~m}, 1.80 \mathrm{H}), 1.70-1.65(\mathrm{~m}, 0.10 \mathrm{H}), 1.58(\mathrm{dd}, J=13.2,3.6 \mathrm{~Hz}$, $0.20 \mathrm{H}), 1.52-1.45(\mathrm{~m}, 0.90 \mathrm{H}), 1.30-1.17(\mathrm{~m}, 3 \mathrm{H}) ;{ }^{13} \mathrm{C} \mathrm{NMR}\left(100 \mathrm{MHz}, \mathrm{CDCl}_{3}\right) \delta$ (major + minor) $175.7,162.0,161.6,154.9,144.0,133.0,131.1,131.0,128.8,128.7,126.3,126.1,125.9$, $122.8,117.2,106.6,95.1,94.6,90.7,90.1,48.4,44.3,41.1,39.4,37.7,36.8,36.6,36.0,29.3,28.0$, 23.2, 22.3, 21.8. HRMS (ESI-TOF): calcd. for $\mathrm{C}_{25} \mathrm{H}_{23} \mathrm{O}_{3}[\mathrm{M}+\mathrm{H}]^{+}$371.1642; found 371.1645.

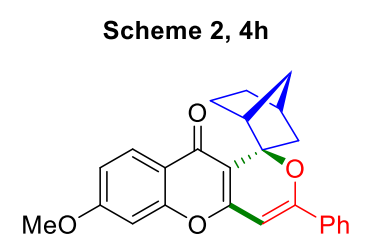

Compound 4h: purification by flash column chromatography on silica gel (eluent: petroleum ether/ethyl acetate/dichloromethane $=20: 1: 0.1-3: 1: 0.1) ;$ yellow solid, $126.7 \mathrm{mg}, 82 \%$ yield, $\mathrm{mp}$ $163.7-165.2{ }^{\circ} \mathrm{C} ; 88: 12 \mathrm{dr} ;{ }^{1} \mathrm{H}$ NMR $\left(400 \mathrm{MHz}, \mathrm{CDCl}_{3}\right) \delta$ (major + minor) $8.10(\mathrm{~d}, J=8.8 \mathrm{~Hz}$, $1 \mathrm{H}), 7.85-7.82(\mathrm{~m}, 0.24 \mathrm{H}), 7.80-7.77(\mathrm{~m}, 1.76 \mathrm{H}), 7.46-7.44(\mathrm{~m}, 3 \mathrm{H}), 6.91(\mathrm{dd}, J=8.8,2.4$ $\mathrm{Hz}, 1 \mathrm{H}), 6.79(\mathrm{~d}, J=2.4 \mathrm{~Hz}, 1 \mathrm{H}), 6.31(\mathrm{~s}, 0.12 \mathrm{H}), 6.25(\mathrm{~s}, 0.88 \mathrm{H}), 3.94-3.92(\mathrm{~m}, 0.12 \mathrm{H}), 3.89(\mathrm{~s}$, $3 \mathrm{H}), 3.81(\mathrm{dd}, J=13.2,2.4 \mathrm{~Hz}, 0.88 \mathrm{H}), 2.85(\mathrm{~s}, 0.88 \mathrm{H}), 2.76(\mathrm{~s}, 0.12 \mathrm{H}), 2.50(\mathrm{~s}, 1 \mathrm{H}), 2.17(\mathrm{~d}, J=$ $10.4 \mathrm{~Hz}, 0.12 \mathrm{H}), 1.97(\mathrm{~d}, J=9.6 \mathrm{~Hz}, 0.88 \mathrm{H}), 1.84-1.75(\mathrm{~m}, 1.76 \mathrm{H}), 1.68-1.64(\mathrm{~m}, 0.12 \mathrm{H}), 1.57$ $(\mathrm{dd}, J=13.2,3.2 \mathrm{~Hz}, 0.24 \mathrm{H}), 1.51-1.45(\mathrm{~m}, 0.88 \mathrm{H}), 1.30-1.16(\mathrm{~m}, 3 \mathrm{H}) ;{ }^{13} \mathrm{C}$ NMR $(100 \mathrm{MHz}$, $\left.\mathrm{CDCl}_{3}\right) \delta$ (major + minor) $175.4,163.4,161.7,161.4,156.4,133.0,131.0,130.9,128.8,128.8$, $127.5,126.3,125.9,119.0,113.5,110.1,106.5,100.0,95.0,94.5,90.7,90.1,55.9,48.4,44.3,41.1$, 39.4, 37.7, 36.7, 36.6, 36.0, 29.3, 28.0, 23.2, 22.3. HRMS (ESI-TOF): calcd. for $\mathrm{C}_{25} \mathrm{H}_{23} \mathrm{O}_{4}[\mathrm{M}+$ $\mathrm{H}]^{+}$387.1591; found 387.1595 .

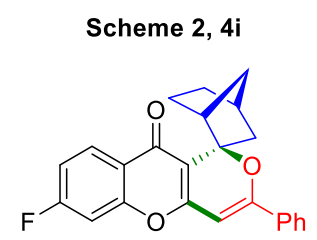

Compound 4i: purification by flash column chromatography on silica gel (eluent: petroleum 
ether/ethyl acetate/dichloromethane $=20: 1: 0.1-3: 1: 0.1)$; yellow solid, $124.9 \mathrm{mg}, 83 \%$ yield, $\mathrm{mp}$ 180.9 - $182.7^{\circ} \mathrm{C} ; 90: 10 \mathrm{dr} ;{ }^{1} \mathrm{H}$ NMR $\left(400 \mathrm{MHz}, \mathrm{CDCl}_{3}\right) \delta$ (major + minor) $8.22-8.16(\mathrm{~m}, 1 \mathrm{H})$, $7.84-7.82(\mathrm{~m}, 0.20 \mathrm{H}), 7.81-7.76(\mathrm{~m}, 1.80 \mathrm{H}), 7.48-7.42(\mathrm{~m}, 3 \mathrm{H}), 7.08-7.04(\mathrm{~m}, 2 \mathrm{H}), 6.32(\mathrm{~s}$, $0.10 \mathrm{H}), 6.26(\mathrm{~s}, 0.90 \mathrm{H}), 3.89-3.85(\mathrm{~m}, 0.10 \mathrm{H}), 3.78(\mathrm{dd}, J=13.6,2.2 \mathrm{~Hz}, 0.90 \mathrm{H}), 2.85(\mathrm{~d}, J=$ $4.4 \mathrm{~Hz}, 0.90 \mathrm{H}), 2.77$ (d, $J=3.6 \mathrm{~Hz}, 0.10 \mathrm{H}), 2.52-2.46(\mathrm{~m}, 1 \mathrm{H}), 2.14(\mathrm{~d}, J=10.2 \mathrm{~Hz}, 0.10 \mathrm{H})$, $1.97(\mathrm{~d}, J=9.6 \mathrm{~Hz}, 0.90 \mathrm{H}), 1.85-1.75(\mathrm{~m}, 1.80 \mathrm{H}), 1.69-1.66(\mathrm{~m}, 0.10 \mathrm{H}), 1.58(\mathrm{dd}, J=13.4,2.6$ $\mathrm{Hz}, 0.20 \mathrm{H}), 1.51-1.45(\mathrm{~m}, 0.90 \mathrm{H}), 1.30-1.17(\mathrm{~m}, 3 \mathrm{H}) ;{ }^{13} \mathrm{C} \mathrm{NMR}\left(100 \mathrm{MHz}, \mathrm{CDCl}_{3}\right) \delta($ major + minor) $174.8,165.2(\mathrm{~d}, J=252.1 \mathrm{~Hz}), 162.4,162.1,155.7(\mathrm{~d}, J=13.2 \mathrm{~Hz}), 132.8,131.9,131.3$, 131.2, 128.9, 128.8, 128.6 (d, $J=10.4 \mathrm{~Hz}), 126.3,126.0,121.9$ (d, $J=2.5 \mathrm{~Hz}), 113.2$ (d, $J=22.4$ Hz), 106.5, 104.1 (d, $J=25.1 \mathrm{~Hz}), 94.7,94.2,90.7,90.1,48.4,44.3,41.1,39.4,37.7,36.7,36.5$, 36.0, 29.3, 27.9, 23.1, 22.2. HRMS (ESI-TOF): calcd. for $\mathrm{C}_{24} \mathrm{H}_{20} \mathrm{FO}_{3}[\mathrm{M}+\mathrm{H}]^{+} 375.1391$; found 375.1401.

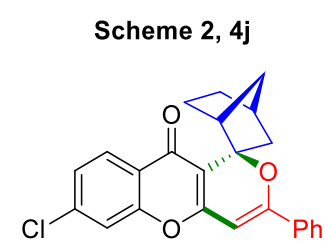

Compound $\mathbf{4 j}$ : purification by flash column chromatography on silica gel (eluent: petroleum ether/ethyl acetate/dichloromethane $=20: 1: 0.1-3: 1: 0.1)$; yellow solid, $130.9 \mathrm{mg}, 84 \%$ yield, $\mathrm{mp}$ 182.9 - $184.7{ }^{\circ} \mathrm{C} ; 90: 10 \mathrm{dr} ;{ }^{1} \mathrm{H}$ NMR $\left(400 \mathrm{MHz}, \mathrm{CDCl}_{3}\right) \delta$ (major + minor) $8.10(\mathrm{~d}, J=8.4 \mathrm{~Hz}$, 1H), $7.81(\mathrm{~d}, J=7.4 \mathrm{~Hz}, 0.20 \mathrm{H}), 7.77(\mathrm{~d}, J=7.8 \mathrm{~Hz}, 1.80 \mathrm{H}), 7.47-7.42(\mathrm{~m}, 3 \mathrm{H}), 7.36-7.35(\mathrm{~m}$, 1H), $7.29-7.26(\mathrm{~m}, 1 \mathrm{H}), 6.29(\mathrm{~s}, 0.10 \mathrm{H}), 6.23(\mathrm{~s}, 0.90 \mathrm{H}), 3.88-3.83(\mathrm{~m}, 0.10 \mathrm{H}), 3.77(\mathrm{dd}, J=$ 13.2, $2.4 \mathrm{~Hz}, 0.90 \mathrm{H}), 2.84(\mathrm{~d}, J=4.4 \mathrm{~Hz}, 0.90 \mathrm{H}), 2.76(\mathrm{~d}, J=4.4 \mathrm{~Hz}, 0.10 \mathrm{H}), 2.50-2.47(\mathrm{~m}, 1 \mathrm{H})$, $2.13(\mathrm{~d}, J=9.6 \mathrm{~Hz}, 0.10 \mathrm{H}), 1.96(\mathrm{~d}, J=9.6 \mathrm{~Hz}, 0.90 \mathrm{H}), 1.85-1.77(\mathrm{~m}, 1.80 \mathrm{H}), 1.68-1.65(\mathrm{~m}$, $0.10 \mathrm{H}), 1.58(\mathrm{dd}, J=13.6,3.2 \mathrm{~Hz}, 0.20 \mathrm{H}), 1.51-1.46(\mathrm{~m}, 0.90 \mathrm{H}), 1.30-1.17(\mathrm{~m}, 3 \mathrm{H}) ;{ }^{13} \mathrm{C} \mathrm{NMR}$ $\left(100 \mathrm{MHz}, \mathrm{CDCl}_{3}\right) \delta$ (major + minor) $174.7,174.1,162.8,162.6,161.9,161.8,154.8,154.8$, 138.6, 138.5, 132.7, 131.8, 131.3, 131.2, 128.8, 128.7, 127.5, 126.3, 125.9, 125.4, 125.3, 123.6, 117.4, 109.9, 106.7, 94.6, 94.1, 90.6, 90.1, 48.4, 44.3, 41.0, 39.4, 37.7, 36.7, 36.5, 35.9, 29.2, 27.9, 23.1, 22.2. HRMS (ESI-TOF): calcd. for $\mathrm{C}_{24} \mathrm{H}_{20} \mathrm{ClO}_{3}[\mathrm{M}+\mathrm{H}]^{+}$391.1095; found 391.1105. 


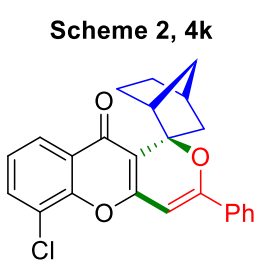

Compound 4k: purification by flash column chromatography on silica gel (eluent: petroleum ether/ethyl acetate/dichloromethane $=$ 20:1:0.1 $-3: 1: 0.1)$; yellow solid, $109.4 \mathrm{mg}, 70 \%$ yield, $\mathrm{mp}$ 171.3 - $172.0{ }^{\circ} \mathrm{C} ; 86: 14 \mathrm{dr} ;{ }^{1} \mathrm{H}$ NMR $\left(400 \mathrm{MHz}, \mathrm{CDCl}_{3}\right) \delta$ (major + minor) $8.10(\mathrm{~d}, J=8.0 \mathrm{~Hz}$, 1H), $7.86(\mathrm{~d}, J=8.0 \mathrm{~Hz}, 0.28 \mathrm{H}), 7.81(\mathrm{~d}, J=7.8 \mathrm{~Hz}, 1.72 \mathrm{H}), 7.64(\mathrm{~d}, J=7.8 \mathrm{~Hz}, 1 \mathrm{H}), 7.47-7.43$ (m, 3H), $7.29-7.25(\mathrm{~m}, 1 \mathrm{H}), 6.44$ (s, 0.14H), 6.38 (s, 0.86H), $3.86(\mathrm{dd}, J=13.2,2.4 \mathrm{~Hz}, 0.14 \mathrm{H})$, $3.78(\mathrm{~d}, J=13.2 \mathrm{~Hz}, 0.86 \mathrm{H}), 2.87(\mathrm{~d}, J=4.4 \mathrm{~Hz}, 0.86 \mathrm{H}), 2.80(\mathrm{~d}, J=3.6 \mathrm{~Hz}, 0.14 \mathrm{H}), 2.52-2.47$ (m, 1H), $2.13(\mathrm{~d}, \mathrm{~J}=10.2 \mathrm{~Hz}, 0.14 \mathrm{H}), 1.98(\mathrm{~d}, J=9.6 \mathrm{~Hz}, 0.86 \mathrm{H}), 1.85(\mathrm{~d}, J=14.0 \mathrm{~Hz}, 0.86 \mathrm{H})$, $1.80-1.75(\mathrm{~m}, 0.86 \mathrm{H}), 1.70-1.66(\mathrm{~m}, 0.14 \mathrm{H}), 1.60(\mathrm{~d}, \mathrm{~J}=13.6 \mathrm{~Hz}, 0.14 \mathrm{H}), 1.53-1.43(\mathrm{~m}, 1 \mathrm{H})$, $1.31-1.18(\mathrm{~m}, 3 \mathrm{H}) ;{ }^{13} \mathrm{C} \mathrm{NMR}\left(100 \mathrm{MHz}, \mathrm{CDCl}_{3}\right) \delta$ (major + minor) 174.8, 174.3, 163.1, 162.8, $162.0,161.9,150.5,150.4,133.0,133.0,132.7,131.9,131.4,131.3,128.9,128.8,127.3,126.6$, 126.4, 126.1, 124.8, 124.7, 122.3, 109.8, 106.6, 94.8, 94.3, 90.7, 90.2, 48.4, 44.3, 41.1, 39.4, 37.8, 36.7, 36.6, 36.0, 29.3, 27.9, 23.2, 22.2. HRMS (ESI-TOF): calcd. for $\mathrm{C}_{24} \mathrm{H}_{20} \mathrm{ClO}_{3}[\mathrm{M}+\mathrm{H}]^{+}$ 391.1095; found 391.1093.

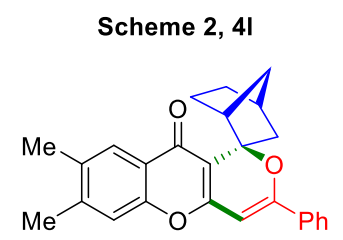

Compound 4l: purification by flash column chromatography on silica gel (eluent: petroleum ether/ethyl acetate/dichloromethane $=20: 1: 0.1-3: 1: 0.1$ ); yellow solid, $123.0 \mathrm{mg}, 80 \%$ yield, $\mathrm{mp}$ 179.2 - $180.6{ }^{\circ} \mathrm{C} ; 90: 10 \mathrm{dr} ;{ }^{1} \mathrm{H}$ NMR $\left(400 \mathrm{MHz}, \mathrm{CDCl}_{3}\right) \delta$ (major + minor) 7.92 (s, 0.90H), 7.91 (s, 0.10), $7.85-7.81(\mathrm{~m}, 0.20 \mathrm{H}), 7.79-7.77(\mathrm{~m}, 1.80 \mathrm{H}), 7.66-7.58(\mathrm{~m}, 0.30 \mathrm{H}), 7.44-7.40(\mathrm{~m}$, 2.70H), 7.13 (s, 0.90H), $7.12(\mathrm{~s}, 0.10 \mathrm{H}), 6.31$ (s, 0.10H), 6.25 (s, 0.90H), 3.93 (ddd, $J=13.2,4.8$, $2.8 \mathrm{~Hz}, 0.10 \mathrm{H}), 3.84(\mathrm{dd}, J=13.2,2.8 \mathrm{~Hz}, 0.90 \mathrm{H}), 2.85-2.84(\mathrm{~m}, 0.90 \mathrm{H}), 2.76-2.75(\mathrm{~m}, 0.10 \mathrm{H})$, $2.51-2.47(\mathrm{~m}, 1 \mathrm{H}), 2.35(\mathrm{~s}, 3 \mathrm{H}), 2.32(\mathrm{~s}, 3 \mathrm{H}), 2.18(\mathrm{~d}, J=9.2 \mathrm{~Hz}, 0.10 \mathrm{H}), 1.97(\mathrm{~d}, J=9.2 \mathrm{~Hz}$, $0.90 \mathrm{H}), 1.85-1.78(\mathrm{~m}, 1.80 \mathrm{H}), 1.69-1.64(\mathrm{~m}, 0.10 \mathrm{H}), 1.57(\mathrm{dd}, J=13.2,3.2 \mathrm{~Hz}, 0.20 \mathrm{H}), 1.52-$ $1.43(\mathrm{~m}, 0.90 \mathrm{H}), 1.29-1.16(\mathrm{~m}, 3 \mathrm{H}) ;{ }^{13} \mathrm{C} \mathrm{NMR}\left(100 \mathrm{MHz}, \mathrm{CDCl}_{3}\right) \delta$ (major + minor) 175.8, 
175.2, 161.7, 161.4, 153.3, 143.0, 133.7, 133.0, 131.0, 130.9, 128.8, 128.7, 126.2, 125.9, 122.9, 117.6, 106.6, 95.1, 94.7, 90.7, 90.1, 48.5, 44.4, 41.1, 39.4, 37.7, 36.8, 36.57, 36.0, 29.3, 27.9, 23.2, 22.2, 20.4, 19.5, 19.4. HRMS (ESI-TOF): calcd. for $\mathrm{C}_{26} \mathrm{H}_{25} \mathrm{O}_{3}[\mathrm{M}+\mathrm{H}]^{+}$385.1798; found 385.1804.

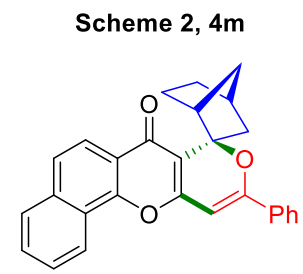

Compound $\mathbf{4 m}$ : purification by flash column chromatography on silica gel (eluent: petroleum ether/ethyl acetate $=20: 1-4: 1$ ); yellow solid, $99.2 \mathrm{mg}, 61 \%$ yield, $\mathrm{mp} 215.2-216.7^{\circ} \mathrm{C} ; 95: 5 \mathrm{dr}$; ${ }^{1} \mathrm{H}$ NMR $\left(400 \mathrm{MHz}, \mathrm{CDCl}_{3}\right) \delta$ (major + minor) $8.50-8.46(\mathrm{~m}, 1 \mathrm{H}), 8.17(\mathrm{dd}, J=8.8,4.8 \mathrm{~Hz}, 1 \mathrm{H})$, $7.90-7.83(\mathrm{~m}, 3 \mathrm{H}), 7.70(\mathrm{dd}, J=8.8,4.8 \mathrm{~Hz}, 1 \mathrm{H}), 7.66-7.63(\mathrm{~m}, 2 \mathrm{H}), 7.48-7.46(\mathrm{~m}, 3 \mathrm{H}), 6.50$ $(\mathrm{d}, J=4.8 \mathrm{~Hz}, 0.05 \mathrm{H}), 6.44(\mathrm{~d}, J=4.8 \mathrm{~Hz}, 0.95 \mathrm{H}), 3.87(\mathrm{~d}, J=13.2 \mathrm{~Hz}, 1 \mathrm{H}), 2.91(\mathrm{~s}, 1 \mathrm{H}), 2.54(\mathrm{~s}$, 1H), $2.01(\mathrm{~d}, J=9.6 \mathrm{~Hz}, 1 \mathrm{H}), 1.89-1.85(\mathrm{~m}, 2 \mathrm{H}), 1.52-1.50(\mathrm{~m}, 1 \mathrm{H}), 1.28-1.19(\mathrm{~m}, 3 \mathrm{H}) ;{ }^{13} \mathrm{C}$ NMR $\left(100 \mathrm{MHz}, \mathrm{CDCl}_{3}\right) \delta$ (major + minor) 175.6, 161.8, 161.1, 151.8, 135.6, 133.0, 131.0, 128.9, 128.8, 128.1, 126.9, 126.3, 124.6, 123.9, 122.1, 121.5, 121.3, 107.9, 94.4, 90.7, 44.3, 39.4, 36.8, 36.0, 28.0, 22.3. HRMS (ESI-TOF): calcd. for $\mathrm{C}_{28} \mathrm{H}_{23} \mathrm{O}_{3}[\mathrm{M}+\mathrm{H}]^{+} 407.1642$; found 407.1645.

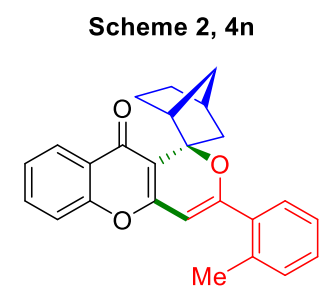

Compound 4n: purification by flash column chromatography on silica gel (eluent: petroleum ether/ethyl acetate/dichloromethane $=20: 1: 0.1-3: 1: 0.1) ;$ yellow solid, $115.3 \mathrm{mg}, 78 \%$ yield, $\mathrm{mp}$ $117.8-119.2{ }^{\circ} \mathrm{C} ; 89: 11 \mathrm{dr} ;{ }^{1} \mathrm{H}$ NMR $\left(400 \mathrm{MHz}, \mathrm{CDCl}_{3}\right) \delta$ (major + minor) $8.21(\mathrm{~d}, J=8.0 \mathrm{~Hz}$, 1H), $7.60-7.55(\mathrm{~m}, 1 \mathrm{H}), 7.52(\mathrm{~d}, J=7.6 \mathrm{~Hz}, 1 \mathrm{H}), 7.38-7.31(\mathrm{~m}, 3 \mathrm{H}), 7.28-7.24(\mathrm{~m}, 1.78 \mathrm{H})$, $7.12-7.08(\mathrm{~m}, 0.22 \mathrm{H}), 5.96(\mathrm{~d}, J=1.8 \mathrm{~Hz}, 0.11 \mathrm{H}), 5.89(\mathrm{~d}, J=1.8 \mathrm{~Hz}, 0.89 \mathrm{H}), 3.88-3.85(\mathrm{~m}$, $0.11 \mathrm{H}), 3.81(\mathrm{~d}, J=13.2 \mathrm{~Hz}, 0.89 \mathrm{H}), 2.99(\mathrm{~s}, 0.89 \mathrm{H}), 2.87(\mathrm{~s}, 0.11 \mathrm{H}), 2.52(\mathrm{~s}, 2.67 \mathrm{H}), 2.50(\mathrm{~s}$, $0.33 \mathrm{H}), 2.46$ (s, 0.89H), $2.43(\mathrm{~s}, 0.11 \mathrm{H}), 2.17$ (d, $J=10.4 \mathrm{~Hz}, 0.11 \mathrm{H}), 1.93(\mathrm{~d}, J=9.6 \mathrm{~Hz}, 0.89 \mathrm{H})$, 
$1.85-1.74(\mathrm{~m}, 2 \mathrm{H}), 1.52-1.43(\mathrm{~m}, 1 \mathrm{H}), 1.29-1.26(\mathrm{~m}, 2 \mathrm{H}), 1.20(\mathrm{~d}, J=9.6 \mathrm{~Hz}, 1 \mathrm{H}) ;{ }^{13} \mathrm{C}$ NMR $\left(100 \mathrm{MHz}, \mathrm{CDCl}_{3}\right) \delta$ (major + minor) $175.7,175.2,165.6,164.3,161.4,154.7,136.5,136.1$, 133.9, 133.6, 132.8, 132.7, 132.1, 131.9, 131.5, 131.3, 130.0, 129.8, 128.9, 128.1, 127.9, 126.1, 126.0, 125.2, 124.6, 117.4, 109.2, 105.8, 99.7, 99.0, 91.0, 90.3, 67.4, 48.3, 44.7, 41.0, 39.5, 37.5, 36.8, 36.5, 36.2, 29.3, 27.9, 27.2, 23.5, 22.2, 21.5, 21.3. HRMS (ESI-TOF): calcd. for $\mathrm{C}_{25} \mathrm{H}_{23} \mathrm{O}_{3}$ $[\mathrm{M}+\mathrm{H}]^{+} 371.1642$; found 371.1653 .

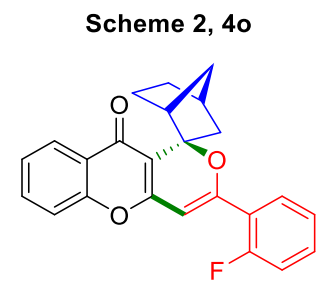

Compound 4o: purification by flash column chromatography on silica gel (eluent: petroleum ether/ethyl acetate/dichloromethane $=20: 1: 0.1-3: 1: 0.1)$; yellow solid, $107.6 \mathrm{mg}, 72 \%$ yield, $\mathrm{mp}$ $149.1-150.6{ }^{\circ} \mathrm{C} ; 88: 12 \mathrm{dr} ;{ }^{1} \mathrm{H}$ NMR $\left(400 \mathrm{MHz}, \mathrm{CDCl}_{3}\right) \delta$ (major + minor) $8.19(\mathrm{~d}, J=7.8 \mathrm{~Hz}$, 1H), $7.95-7.91(\mathrm{~m}, 0.12 \mathrm{H}), 7.85(\mathrm{td}, J=8.0,2.0 \mathrm{~Hz}, 0.88 \mathrm{H}), 7.59(\mathrm{t}, J=7.0 \mathrm{~Hz}, 1 \mathrm{H}), 7.43-7.32$ (m, 3H), $7.26-7.21(\mathrm{~m}, 1 \mathrm{H}), 7.14(\mathrm{dd}, J=12.0,8.0 \mathrm{~Hz}, 1 \mathrm{H}), 6.64(\mathrm{~s}, 0.12 \mathrm{H}), 6.54(\mathrm{~s}, 0.88 \mathrm{H})$, $3.91-3.86(\mathrm{~m}, 0.12 \mathrm{H}), 3.80(\mathrm{dd}, J=13.6,2.4 \mathrm{~Hz}, 0.88 \mathrm{H}), 2.90(\mathrm{~d}, J=4.4 \mathrm{~Hz}, 0.88 \mathrm{H}), 2.80(\mathrm{~d}, J$ $=4.0 \mathrm{~Hz}, 0.12 \mathrm{H}), 2.50(\mathrm{~s}, 1 \mathrm{H}), 2.18(\mathrm{~d}, J=10.0 \mathrm{~Hz}, 0.12 \mathrm{H}), 1.92(\mathrm{~d}, J=10.0 \mathrm{~Hz}, 0.88 \mathrm{H}), 1.83-$ $1.78(\mathrm{~m}, 1.76 \mathrm{H}), 1.67-1.64(\mathrm{~m}, 0.12 \mathrm{H}), 1.58-1.54(\mathrm{~m}, 0.24 \mathrm{H}), 1.51-1.45(\mathrm{~m}, 0.88 \mathrm{H}), 1.30-$ $1.19(\mathrm{~m}, 3 \mathrm{H}) ;{ }^{13} \mathrm{C} \mathrm{NMR}\left(100 \mathrm{MHz}, \mathrm{CDCl}_{3}\right) \delta$ (major + minor) 175.8, 175.2, 161.3, 161.2, $161.1(\mathrm{~d}$, $J=253.5 \mathrm{~Hz}), 156.5(\mathrm{~d}, J=4.3 \mathrm{~Hz}), 154.9,154.8,132.9,132.2,132.07(\mathrm{~d}, J=9.1 \mathrm{~Hz}), 128.9(\mathrm{~d}, J$ = $1.9 \mathrm{~Hz}), 128.1(\mathrm{~d}, J=1.9 \mathrm{~Hz}), 126.1,125.9,125.1,124.7,124.6,124.5(\mathrm{~d}, J=3.6 \mathrm{~Hz}), 121.2(\mathrm{~d}$, $J=9.4 \mathrm{~Hz}), 117.6,116.6(\mathrm{~d}, J=23.1 \mathrm{~Hz}), 116.5(\mathrm{~d}, J=22.9 \mathrm{~Hz}), 107.4,101.2(\mathrm{~d}, J=18.6 \mathrm{~Hz})$, $100.4(\mathrm{~d}, J=16.4 \mathrm{~Hz}), 90.4,89.8,48.3,44.3,41.0,39.4,37.7,36.8,36.6,36.0,29.3,27.9,23.4$, 22.2. HRMS (ESI-TOF): calcd. for $\mathrm{C}_{24} \mathrm{H}_{20} \mathrm{FO}_{3}[\mathrm{M}+\mathrm{H}]^{+}$375.1391; found 375.1385 . 


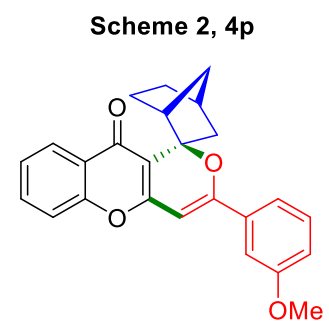

Compound 4p: purification by flash column chromatography on silica gel (eluent: petroleum ether/ethyl acetate/dichloromethane $=20: 1: 0.1-3: 1: 0.1)$, yellow solid, $117.5 \mathrm{mg}, 76 \%$ yield, $\mathrm{mp}$ $163.7-165.6{ }^{\circ} \mathrm{C} ; 88: 12 \mathrm{dr} ;{ }^{1} \mathrm{H}$ NMR $\left(400 \mathrm{MHz}, \mathrm{CDCl}_{3}\right) \delta$ (major + minor) $8.19(\mathrm{~d}, J=8.0 \mathrm{~Hz}$, 1H), $7.61-7.54(\mathrm{~m}, 1 \mathrm{H}), 7.41-7.29(\mathrm{~m}, 5 \mathrm{H}), 6.99(\mathrm{dt}, J=7.6,2.0 \mathrm{~Hz}, 1 \mathrm{H}), 6.33(\mathrm{~s}, 0.12 \mathrm{H}), 6.27$ (s, $0.88 \mathrm{H}), 3.86(\mathrm{~s}, 3 \mathrm{H}), 3.82(\mathrm{~d}, J=14.4 \mathrm{~Hz}, 1 \mathrm{H}), 2.85(\mathrm{~s}, 0.88 \mathrm{H}), 2.78(\mathrm{~s}, 0.12 \mathrm{H}), 2.50(\mathrm{~s}, 1 \mathrm{H})$, $2.17(\mathrm{~d}, J=10.2 \mathrm{~Hz}, 0.12 \mathrm{H}), 1.96(\mathrm{~d}, J=10.2 \mathrm{~Hz}, 0.88 \mathrm{H}), 1.85-1.77(\mathrm{~m}, 2 \mathrm{H}), 1.53-1.42(\mathrm{~m}$, $1 \mathrm{H}), 1.30-1.17(\mathrm{~m}, 3 \mathrm{H}) ;{ }^{13} \mathrm{C} \mathrm{NMR}\left(100 \mathrm{MHz}, \mathrm{CDCl}_{3}\right) \delta$ (major + minor) 175.6, 161.9, 161.7, $159.8,154.7,134.3,132.8,129.8,129.7,126.1,125.1,124.6,118.7,118.4,117.4,116.7,116.5$, $111.8,111.3,106.8,95.3,94.8,90.7,55.4,48.4,44.3,41.1,39.3,37.8,36.7,36.5,36.0,29.2,27.9$, 23.2, 22.2. HRMS (ESI-TOF): calcd. for $\mathrm{C}_{25} \mathrm{H}_{23} \mathrm{O}_{4}[\mathrm{M}+\mathrm{H}]^{+}$387.1591; found 387.1596.

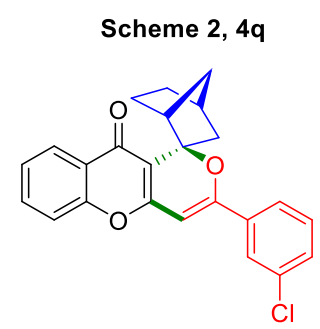

Compound 4q: purification by flash column chromatography on silica gel (eluent: petroleum ether/ethyl acetate/dichloromethane $=20: 1: 0.1-3: 1: 0.1) ;$ yellow solid, $127.5 \mathrm{mg}, 82 \%$ yield, $\mathrm{mp}$ $126.7-128.5{ }^{\circ} \mathrm{C} ; 89: 11 \mathrm{dr} ;{ }^{1} \mathrm{H}$ NMR $\left(400 \mathrm{MHz}, \mathrm{CDCl}_{3}\right) \delta$ (major + minor) $8.18(\mathrm{dd}, J=8.0,2.0$ Hz, 1H), $7.77(\mathrm{~s}, 0.11 \mathrm{H}), 7.73(\mathrm{~s}, 0.89 \mathrm{H}), 7.64(\mathrm{~d}, J=7.6 \mathrm{~Hz}, 1 \mathrm{H}), 7.61-7.54(\mathrm{~m}, 1 \mathrm{H}), 7.47-$ $7.31(\mathrm{~m}, 4 \mathrm{H}), 6.31(\mathrm{~s}, 0.11 \mathrm{H}), 6.26(\mathrm{~s}, 0.89 \mathrm{H}), 3.89(\mathrm{~d}, J=13.6 \mathrm{~Hz}, 0.11 \mathrm{H}), 3.80(\mathrm{~d}, J=13.6 \mathrm{~Hz}$, $0.89 \mathrm{H}), 2.82(\mathrm{~s}, 0.89 \mathrm{H}), 2.75(\mathrm{~s}, 0.11 \mathrm{H}), 2.58(\mathrm{~s}, 0.11 \mathrm{H}), 2.50(\mathrm{~s}, 0.89 \mathrm{H}), 2.15(\mathrm{~d}, J=10.0 \mathrm{~Hz}$ $0.11 \mathrm{H}), 1.92(\mathrm{~d}, J=10.0 \mathrm{~Hz}, 0.89 \mathrm{H}), 1.85-1.75(\mathrm{~m}, 2 \mathrm{H}), 1.51-1.44(\mathrm{~m}, 1 \mathrm{H}), 1.28-1.16(\mathrm{~m}$, $3 \mathrm{H}) ;{ }^{13} \mathrm{C} \mathrm{NMR}\left(100 \mathrm{MHz}, \mathrm{CDCl}_{3}\right) \delta$ (major + minor) 175.6, 161.2, 160.5, 154.8, 134.9, 134.7, $132.9,130.8,130.0,126.2,126.1,125.1,124.7,124.3,123.9,117.4,107.2,96.0,95.4,91.0,65.9$, 48.4, 44.4, 41.0, 39.4, 37.7, 36.7, 36.6, 36.0, 29.3, 27.8, 27.3, 23.3. HRMS (ESI-TOF): calcd. for $\mathrm{C}_{24} \mathrm{H}_{20} \mathrm{ClO}_{3}[\mathrm{M}+\mathrm{H}]^{+}$391.1095; found 391.1103. 


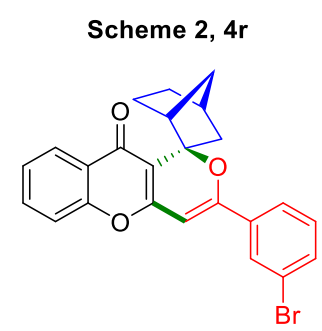

Compound 4r: purification by flash column chromatography on silica gel (eluent: petroleum ether/ethyl acetate/dichloromethane $=20: 1: 0.1-3: 1: 0.1) ;$ yellow solid, $125.4 \mathrm{mg}, 72 \%$ yield, $\mathrm{mp}$ $79.9-81.3{ }^{\circ} \mathrm{C} ; 88: 12 \mathrm{dr} ;{ }^{1} \mathrm{H} \mathrm{NMR}\left(400 \mathrm{MHz}, \mathrm{CDCl}_{3}\right) \delta$ (major + minor) $8.16(\mathrm{~d}, J=8.0 \mathrm{~Hz}, 1 \mathrm{H})$, $7.90(\mathrm{~s}, 0.12 \mathrm{H}), 7.86(\mathrm{~s}, 0.88 \mathrm{H}), 7.72(\mathrm{~d}, J=8.0 \mathrm{~Hz}, 0.12 \mathrm{H}), 7.67$ (d, $J=8.0 \mathrm{~Hz}, 0.88 \mathrm{H}), 7.60-$ 7.49 (m, 2H), $7.35-7.25$ (m, 3H), 6.29 (s, 0.12H), 6.23 (s, 0.88H), 3.87 (d, J = 12.2 Hz, 0.12H), $3.78(\mathrm{~d}, J=13.4 \mathrm{~Hz}, 0.88 \mathrm{H}), 2.80(\mathrm{~s}, 0.88 \mathrm{H}), 2.73(\mathrm{~s}, 0.12 \mathrm{H}), 2.49(\mathrm{~s}, 1 \mathrm{H}), 2.14(\mathrm{~d}, J=10.0 \mathrm{~Hz}$, $0.12 \mathrm{H}), 1.90(\mathrm{~d}, J=10.0 \mathrm{~Hz}, 0.88 \mathrm{H}), 1.84-1.71(\mathrm{~m}, 2 \mathrm{H}), 1.53-1.42(\mathrm{~m}, 1 \mathrm{H}), 1.24-1.16(\mathrm{~m}$, $3 \mathrm{H}) ;{ }^{13} \mathrm{C}$ NMR $\left(100 \mathrm{MHz}, \mathrm{CDCl}_{3}\right) \delta$ (major + minor) 175.6, 175.0, 161.1, 161.0, 160.5, 160.3, $154.7,154.6,134.9,133.8,133.7,132.9,132.9,130.3,130.2,129.1,128.7,126.1,125.0,124.7$, 124.7, 124.3, 123.0, 122.9, 117.4, 110.3, 107.1, 96.0, 95.4, 90.9, 90.3, 48.4, 44.4, 41.0, 39.3, 37.7, 36.7, 36.5, 36.0, 29.2, 27.8, 27.3, 23.3, 22.2. HRMS (ESI-TOF): calcd. for $\mathrm{C}_{24} \mathrm{H}_{19} \mathrm{BrNaO}_{3}[\mathrm{M}+$ $\mathrm{Na}]^{+}$457.0410; found 457.0411.

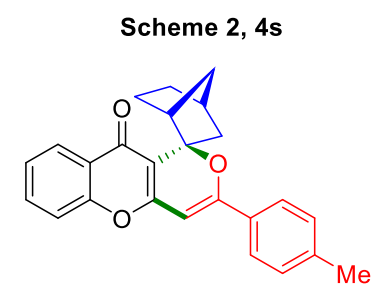

Compound 4s: purification by flash column chromatography on silica gel (eluent: petroleum ether/ethyl acetate $=20: 1-3: 1)$; yellow solid, $120.3 \mathrm{mg}, 81 \%$ yield, $\mathrm{mp} 156.7-158.2{ }^{\circ} \mathrm{C} ; 87: 13$ $\mathrm{dr} ;{ }^{1} \mathrm{H}$ NMR $\left(400 \mathrm{MHz}, \mathrm{CDCl}_{3}\right) \delta$ (major + minor) $8.18(\mathrm{~d}, J=8.8 \mathrm{~Hz}, 1 \mathrm{H}), 7.73(\mathrm{~d}, J=8.0 \mathrm{~Hz}$, $0.26 \mathrm{H}), 7.68(\mathrm{~d}, J=8.0 \mathrm{~Hz}, 1.74 \mathrm{H}), 7.60-7.54(\mathrm{~m}, 1 \mathrm{H}), 7.38-7.31(\mathrm{~m}, 2 \mathrm{H}), 7.25-7.22(\mathrm{~m}, 2 \mathrm{H})$, $6.30(\mathrm{~s}, 0.13 \mathrm{H}), 6.24(\mathrm{~s}, 0.87 \mathrm{H}), 3.94-3.89(\mathrm{~m}, 0.13 \mathrm{H}), 3.81(\mathrm{dd}, J=13.4,2.5 \mathrm{~Hz}, 0.87 \mathrm{H}), 2.85$ $(\mathrm{d}, J=4.0 \mathrm{~Hz}, 0.87 \mathrm{H}), 2.76(\mathrm{~d}, J=4.0 \mathrm{~Hz}, 0.13 \mathrm{H}), 2.49$ (s, $1 \mathrm{H}), 2.40$ (s, 3H), 2.15 (d, $J=10.0 \mathrm{~Hz}$, $0.13 \mathrm{H}), 1.96(\mathrm{~d}, J=10.0 \mathrm{~Hz}, 0.87 \mathrm{H}), 1.86-1.75(\mathrm{~m}, 2 \mathrm{H}), 1.57(\mathrm{dd}, J=13.4,3.5 \mathrm{~Hz}, 0.13 \mathrm{H}), 1.51$ - $1.43(\mathrm{~m}, 0.87 \mathrm{H}), 1.28-1.14(\mathrm{~m}, 3 \mathrm{H}) ;{ }^{13} \mathrm{C} \mathrm{NMR}\left(100 \mathrm{MHz}, \mathrm{CDCl}_{3}\right) \delta$ (major + minor) 175.5, $162.4,162.0,154.8,141.6,132.7,132.6,130.1,129.6,129.5,126.3,126.1,125.9,125.1,124.6$, 
117.4, 106.5, 94.2, 93.8, 90.6, 48.4, 44.3, 41.0, 39.4, 37.7, 36.7, 36.6, 36.0, 29.3, 28.0, 23.1, 22.3, 21.6; HRMS (ESI-TOF): calcd. for $\mathrm{C}_{25} \mathrm{H}_{23} \mathrm{O}_{3}[\mathrm{M}+\mathrm{H}]^{+}$371.1642; found 371.1649.

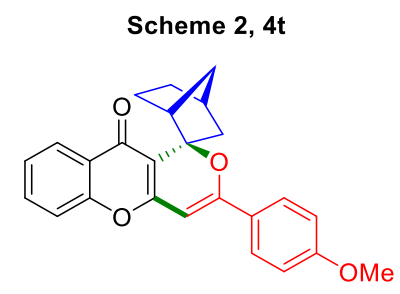

Compound 4t: purification by flash column chromatography on silica gel (eluent: petroleum ether/ethyl acetate $=20: 1-3: 1)$; yellow solid, $109.7 \mathrm{mg}, 71 \%$ yield, mp $171.9-173.4{ }^{\circ} \mathrm{C} ; 88: 12$ $\mathrm{dr} ;{ }^{1} \mathrm{H}$ NMR $\left(400 \mathrm{MHz}, \mathrm{CDCl}_{3}\right) \delta$ (major + minor) $8.19(\mathrm{~d}, J=8.0 \mathrm{~Hz}, 0.88 \mathrm{H}), 7.95-7.21(\mathrm{~m}$, $0.12 \mathrm{H}), 7.81-7.78(\mathrm{~m}, 0.24 \mathrm{H}), 7.74(\mathrm{dd}, J=8.8,2.0 \mathrm{~Hz}, 1.76 \mathrm{H}), 7.60-7.55(\mathrm{~m}, 1 \mathrm{H}), 7.38-7.31$ (m, 2H), $6.96-6.92(\mathrm{~m}, 2 \mathrm{H}), 6.24(\mathrm{~s}, 0.12 \mathrm{H}), 6.18(\mathrm{~s}, 0.88 \mathrm{H}), 3.95-3.91(\mathrm{~m}, 0.12 \mathrm{H}), 3.87-3.85$ (m, 3H), $3.85-3.81(\mathrm{~m}, 0.88 \mathrm{H}), 2.86(\mathrm{~s}, 0.88 \mathrm{H}), 2.77(\mathrm{~s}, 0.12 \mathrm{H}), 2.56(\mathrm{~s}, 0.12 \mathrm{H}), 2.50(\mathrm{~s}, 0.88 \mathrm{H})$ $2.17(\mathrm{~d}, J=10.2 \mathrm{~Hz}, 0.12 \mathrm{H}), 1.96(\mathrm{~d}, J=9.6 \mathrm{~Hz}, 0.88 \mathrm{H}), 1.84-1.78(\mathrm{~m}, 1.76 \mathrm{H}), 1.69-1.68(\mathrm{~m}$, $0.12 \mathrm{H}), 1.59-1.56(\mathrm{~m}, 0.24 \mathrm{H}), 1.49-1.45(\mathrm{~m}, 0.88 \mathrm{H}), 1.28-1.17(\mathrm{~m}, 3 \mathrm{H}) ;{ }^{13} \mathrm{C} \mathrm{NMR}(100 \mathrm{MHz}$ $\left.\mathrm{CDCl}_{3}\right) \delta$ (major + minor) 175.5, 162.3, 162.2, 162.1, 154.8, 132.7, 130.7, 128.1, 127.9, 126.1, 125.5, 125.2, 124.6, 117.4, 114.3, 114.2, 113.8, 106.2, 93.3, 92.9, 90.7, 90.1, 55.6, 48.4, 44.2, 41.1, 39.4, 37.8, 36.8, 36.6, 36.0, 29.8, 29.4, 28.0, 26.5, 23.1, 22.3. HRMS (ESI-TOF): calcd. for $\mathrm{C}_{25} \mathrm{H}_{23} \mathrm{O}_{4}[\mathrm{M}+\mathrm{H}]^{+}$387.1591; found 387.1605.

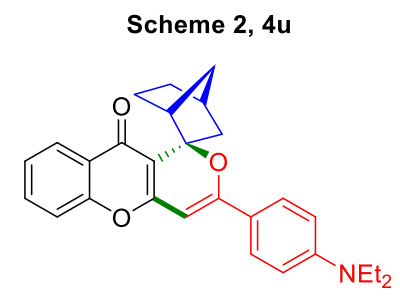

Compound 4u: purification by flash column chromatography on silica gel (eluent: petroleum ether/ethyl acetate $=18: 1-3: 1$ ); yellow solid, $119.7 \mathrm{mg}, 70 \%$ yield, $\mathrm{mp} 96.9-98.6{ }^{\circ} \mathrm{C} ; 88: 12 \mathrm{dr}$; ${ }^{1} \mathrm{H}$ NMR $\left(400 \mathrm{MHz}, \mathrm{CDCl}_{3}\right) \delta$ (major + minor) $8.19(\mathrm{dd}, J=8.0,1.8 \mathrm{~Hz}, 1 \mathrm{H}), 7.73-7.63(\mathrm{~m}, 2 \mathrm{H})$, $7.57-7.53(\mathrm{~m}, 1 \mathrm{H}), 7.36-7.31(\mathrm{~m}, 2 \mathrm{H}), 6.67(\mathrm{~d}, J=8.4 \mathrm{~Hz}, 2 \mathrm{H}), 6.14(\mathrm{~s}, 0.12 \mathrm{H}), 6.10(\mathrm{~s}, 0.88 \mathrm{H})$, $3.98-3.94(\mathrm{~m}, 0.12 \mathrm{H}), 3.85(\mathrm{dd}, J=13.2,2.6 \mathrm{~Hz}, 0.88 \mathrm{H}), 3.42(\mathrm{qd}, J=7.2,2.4 \mathrm{~Hz}, 4 \mathrm{H}), 2.85(\mathrm{~s}$, $0.88 \mathrm{H}), 2.76(\mathrm{~s}, 0.12 \mathrm{H}), 2.49$ (s, 1H), $2.18(\mathrm{~d}, J=9.6 \mathrm{~Hz}, 0.12 \mathrm{H}), 1.97$ (d, $J=9.6 \mathrm{~Hz}, 0.88 \mathrm{H}), 1.85$ 
$-1.72(\mathrm{~m}, 2 \mathrm{H}), 1.51-1.47(\mathrm{~m}, 1 \mathrm{H}), 1.28-1.14(\mathrm{~m}, 9 \mathrm{H}) ;{ }^{13} \mathrm{C} \mathrm{NMR}\left(100 \mathrm{MHz}, \mathrm{CDCl}_{3}\right) \delta($ major + minor) 175.1, 163.5, 163.0, 154.8, 149.9, 132.4, 128.3, 128.1, 126.0, 125.3, 124.3, 119.1, 117.2, 111.0, 105.3, 90.5, 90.3, 48.4, 44.6, 44.0, 39.4, 37.8, 36.8, 36.6, 36.0, 29.4, 28.1, 27.0, 23.0, 22.4, 12.7. HRMS (ESI-TOF): calcd. for $\mathrm{C}_{28} \mathrm{H}_{30} \mathrm{NO}_{3}[\mathrm{M}+\mathrm{H}]^{+} 428.2220$; found 428.2219.

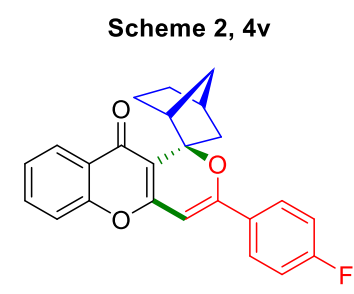

Compound4v: purification by flash column chromatography on silica gel (eluent: petroleum ether/ethyl acetate/dichloromethane $=20: 1: 0.1-3: 1: 0.1)$; yellow solid, $119.8 \mathrm{mg}, 80 \%$ yield, $\mathrm{mp}$ $138.8-140.1{ }^{\circ} \mathrm{C} ; 90: 10 \mathrm{dr} ;{ }^{1} \mathrm{H}$ NMR $\left(400 \mathrm{MHz}, \mathrm{CDCl}_{3}\right) \delta$ (major + minor) $8.19(\mathrm{~d}, J=8.0 \mathrm{~Hz}$, 1H), $7.84-7.76(\mathrm{~m}, 2 \mathrm{H}), 7.58(\mathrm{t}, J=7.6 \mathrm{~Hz}, 1 \mathrm{H}), 7.39-7.31(\mathrm{~m}, 2 \mathrm{H}), 7.16-7.10(\mathrm{~m}, 2 \mathrm{H}), 6.27$ (s, 0.10H), $6.21(\mathrm{~s}, 0.90 \mathrm{H}), 3.91(\mathrm{~d}, J=13.4 \mathrm{~Hz}, 0.10 \mathrm{H}), 3.82(\mathrm{dd}, J=13.4,2.4 \mathrm{~Hz}, 0.90 \mathrm{H}), 2.84$ $(\mathrm{d}, J=4.0 \mathrm{~Hz}, 0.90 \mathrm{H}), 2.77(\mathrm{~d}, J=3.8 \mathrm{~Hz}, 0.10 \mathrm{H}), 2.51-2.47(\mathrm{~m}, 1 \mathrm{H}), 2.17(\mathrm{~d}, J=10.2 \mathrm{~Hz}$, $0.10 \mathrm{H}), 1.93(\mathrm{~d}, J=9.8 \mathrm{~Hz}, 0.90 \mathrm{H}), 1.82-1.80(\mathrm{~m}, 1.80 \mathrm{H}), 1.69-1.67(\mathrm{~m}, 0.10 \mathrm{H}), 1.58-1.55$ $(\mathrm{m}, 0.20 \mathrm{H}), 1.51-1.48(\mathrm{~m}, 0.90 \mathrm{H}), 1.28-1.18(\mathrm{~m}, 3 \mathrm{H}) ;{ }^{13} \mathrm{C} \mathrm{NMR}\left(100 \mathrm{MHz}, \mathrm{CDCl}_{3}\right) \delta($ major + minor) 175.6, 175.0, $164.5(\mathrm{~d}, J=250.9 \mathrm{~Hz}), 161.7,161.6,161.4,161.2,154.8,154.7,132.9$, 132.8, $129.2(\mathrm{~d}, J=3.1 \mathrm{~Hz}), 128.4(\mathrm{~d}, J=8.7 \mathrm{~Hz}), 128.1(\mathrm{~d}, J=8.7 \mathrm{~Hz}), 126.2,125.9,125.1$, 124.7, 117.4, $116.0(\mathrm{~d}, J=21.9 \mathrm{~Hz}), 115.9(\mathrm{~d}, J=21.9 \mathrm{~Hz}), 109.9,106.7,94.8(\mathrm{~d}, J=1.7 \mathrm{~Hz})$, 94.3 (d, $J=1.6 \mathrm{~Hz}), 90.9,90.3,48.4,44.4,41.1,39.4,37.8,36.8,36.6,36.0,29.3$, 27.9, 27.3, 23.2, 22.3. HRMS (ESI-TOF): calcd. for $\mathrm{C}_{24} \mathrm{H}_{20} \mathrm{FO}_{3}[\mathrm{M}+\mathrm{H}]^{+} 375.1391$; found 375.1392 .

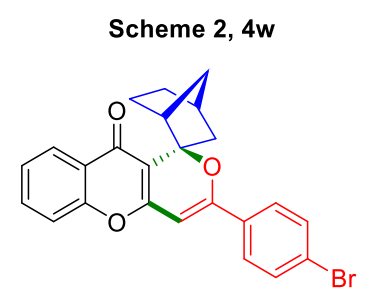

Compound $\mathbf{4 w}$ : purification by flash column chromatography on silica gel (eluent: petroleum ether/ethyl acetate/dichloromethane $=20: 1: 0.1-3: 1: 0.1)$; yellow solid, $142.8 \mathrm{mg}, 82 \%$ yield, $\mathrm{mp}$ $163.9-165.7{ }^{\circ} \mathrm{C} ; 88: 12 \mathrm{dr} ;{ }^{1} \mathrm{H}$ NMR $\left(400 \mathrm{MHz}, \mathrm{CDCl}_{3}\right) \delta$ (major + minor) $8.19(\mathrm{~d}, J=8.0 \mathrm{~Hz}$, 
$0.88 \mathrm{H}), 7.79(\mathrm{dd}, J=19.0,8.0 \mathrm{~Hz}, 0.12 \mathrm{H}), 7.69(\mathrm{~d}, J=8.4 \mathrm{~Hz}, 0.24 \mathrm{H}), 7.64(\mathrm{~d}, J=8.4 \mathrm{~Hz}, 1.76 \mathrm{H})$, $7.61-7.55(\mathrm{~m}, 3 \mathrm{H}), 7.38-7.33(\mathrm{~m}, 2 \mathrm{H}), 6.32(\mathrm{~s}, 0.12 \mathrm{H}), 6.27(\mathrm{~s}, 0.88 \mathrm{H}), 3.90(\mathrm{~d}, J=12.6 \mathrm{~Hz}$, $0.12 \mathrm{H}), 3.81(\mathrm{~d}, J=13.4 \mathrm{~Hz}, 0.88 \mathrm{H}), 2.83(\mathrm{~d}, J=4.4 \mathrm{~Hz}, 0.88 \mathrm{H}), 2.76(\mathrm{~d}, J=3.8 \mathrm{~Hz}, 0.12 \mathrm{H}), 2.51$ $-2.46(\mathrm{~m}, 1 \mathrm{H}), 2.16(\mathrm{~d}, J=10.2 \mathrm{~Hz}, 0.12 \mathrm{H}), 1.91(\mathrm{~d}, J=9.8 \mathrm{~Hz}, 0.88 \mathrm{H}), 1.83-1.77(\mathrm{~m}, 1.76 \mathrm{H})$, $1.68-1.65(\mathrm{~m}, 0.12 \mathrm{H}), 1.58-1.54(\mathrm{~m} \mathrm{~Hz}, 0.24 \mathrm{H}), 1.51-1.45(\mathrm{~m}, 0.88 \mathrm{H}), 1.28-1.17(\mathrm{~m}, 3 \mathrm{H})$; ${ }^{13} \mathrm{C}$ NMR (100 MHz, $\left.\mathrm{CDCl}_{3}\right) \delta$ (major + minor) 175.6, 161.4, 161.0, 154.8, 132.9, 132.1, 132.0, 131.9, 127.7, 127.3, 126.2, 125.5, 125.1, 124.8, 117.4, 107.1, 95.5, 94.9, 90.9, 48.5, 44.4, 41.1, 39.4, 37.7, 36.8, 36.6, 36.0, 29.3, 27.9, 27.3, 23.2, 22.2. HRMS (ESI-TOF): calcd. for $\mathrm{C}_{24} \mathrm{H}_{19} \mathrm{BrNaO}_{3}[\mathrm{M}+\mathrm{Na}]^{+} 457.0410$; found 457.0411.

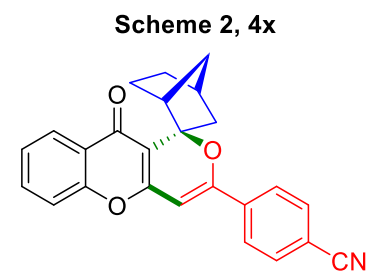

Compound 4x: purification by flash column chromatography on silica gel (eluent: petroleum ether/ethyl acetate/dichloromethane $=18: 1: 0.1-3: 1: 0.1) ;$ yellow solid, $91.5 \mathrm{mg}, 60 \%$ yield, $\mathrm{mp}$ $212.5-214.3{ }^{\circ} \mathrm{C} ; 88: 12 \mathrm{dr} ;{ }^{1} \mathrm{H}$ NMR $\left(400 \mathrm{MHz}, \mathrm{CDCl}_{3}\right) \delta$ (major + minor) $8.20-8.17(\mathrm{~m}, 1 \mathrm{H})$, $7.92-7.86(\mathrm{~m}, 2 \mathrm{H}), 7.73-7.71(\mathrm{~m}, 2 \mathrm{H}), 7.63-7.59(\mathrm{~m}, 1 \mathrm{H}), 7.40-7.33(\mathrm{~m}, 2 \mathrm{H}), 6.43(\mathrm{~d}, J=$ $3.8 \mathrm{~Hz}, 0.12 \mathrm{H}), 6.38(\mathrm{~d}, J=3.8 \mathrm{~Hz}, 0.88 \mathrm{H}), 3.90(\mathrm{~d}, \mathrm{~J}=14.0 \mathrm{~Hz}, 0.12 \mathrm{H}), 3.79(\mathrm{~d}, J=13.6 \mathrm{~Hz}$ 0.88H), 2.82 (s, 0.88H), 2.75 (s, 0.12H), $2.51(\mathrm{~s}, 1 \mathrm{H}), 2.15(\mathrm{~d}, J=10.2 \mathrm{~Hz}, 0.12 \mathrm{H}), 1.89$ (d, $J=9.8$ $\mathrm{Hz}, 0.88 \mathrm{H}), 1.84-1.80(\mathrm{~m}, 2 \mathrm{H}), 1.69-1.67(\mathrm{~m}, 0.12 \mathrm{H}), 1.51-1.45(\mathrm{~m}, 0.88 \mathrm{H}), 1.30-1.20(\mathrm{~m}$, $3 \mathrm{H}) ;{ }^{13} \mathrm{C} \mathrm{NMR}\left(100 \mathrm{MHz}, \mathrm{CDCl}_{3}\right) \delta$ (major + minor) 175.7, 160.6, 160.5, 159.4, 154.8, 154.7, 137.0, 136.2, 133.2, 132.6, 132.5, 126.5, 126.2, 126.1, 125.0, 124.9, 118.4, 117.5, 114.0, 107.8, 97.8, 97.1, 91.1, 90.6, 48.5, 44.6, 41.0, 39.4, 37.7, 36.7, 36.6, 36.0, 29.2, 27.8, 23.4, 22.1, 14.3. HRMS (ESI-TOF): calcd. for $\mathrm{C}_{25} \mathrm{H}_{20} \mathrm{NO}_{3}[\mathrm{M}+\mathrm{H}]^{+} 382.1438$; found 382.1447 . 


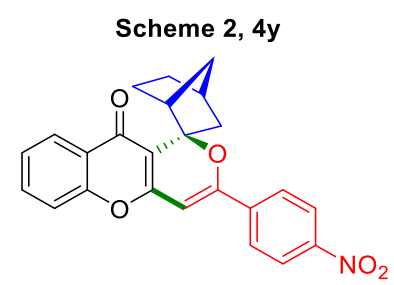

Compound $\mathbf{4 y}$ : purification by flash column chromatography on silica gel (eluent: petroleum ether/ethyl acetate/dichloromethane $=$ 18:1:0.1 $-3: 1: 0.1)$; yellow solid, $99.5 \mathrm{mg}, 62 \%$ yield, $\mathrm{mp}$ $172.0-173.7^{\circ} \mathrm{C} ; 91: 9 \mathrm{dr} ;{ }^{1} \mathrm{H} \mathrm{NMR}\left(400 \mathrm{MHz}, \mathrm{CDCl}_{3}\right) \delta$ (major + minor) $8.27(\mathrm{~d}, J=8.4 \mathrm{~Hz}, 2 \mathrm{H})$, $8.18(\mathrm{~d}, J=8.0 \mathrm{~Hz}, 0.91 \mathrm{H}), 8.06(\mathrm{~d}, J=8.4 \mathrm{~Hz}, 0.09 \mathrm{H}), 7.96(\mathrm{~d}, J=8.4 \mathrm{~Hz}, 0.18 \mathrm{H}), 7.92(\mathrm{~d}, J=$ $8.4 \mathrm{~Hz}, 1.82 \mathrm{H}), 7.61(\mathrm{t}, J=7.6 \mathrm{~Hz}, 1 \mathrm{H}), 7.40-7.33(\mathrm{~m}, 1.82 \mathrm{H}), 7.16-7.12(\mathrm{~m}, 0.18 \mathrm{H}), 6.47(\mathrm{~s}$, 0.09H), $6.42(\mathrm{~s}, 0.91 \mathrm{H}), 3.90(\mathrm{~d}, J=12.4 \mathrm{~Hz}, 0.09 \mathrm{H}), 3.79(\mathrm{dd}, J=13.6,2.4 \mathrm{~Hz}, 0.91 \mathrm{H}), 2.82(\mathrm{~d}, J$ $=4.4 \mathrm{~Hz}, 0.91 \mathrm{H}), 2.75(\mathrm{~d}, J=3.6 \mathrm{~Hz}, 0.09 \mathrm{H}), 2.53-2.48(\mathrm{~m}, 1 \mathrm{H}), 2.15(\mathrm{~d}, J=10.4 \mathrm{~Hz}, 0.09 \mathrm{H})$, $1.89(\mathrm{~d}, J=9.6 \mathrm{~Hz}, 0.91 \mathrm{H}), 1.84-1.75(\mathrm{~m}, 1.82 \mathrm{H}), 1.66-1.64(\mathrm{~m}, 0.09 \mathrm{H}), 1.58-1.54(\mathrm{~m}$, $0.18 \mathrm{H}), 1.52-1.46(\mathrm{~m}, 0.91 \mathrm{H}), 1.26-1.18(\mathrm{~m}, 3 \mathrm{H}) ;{ }^{13} \mathrm{C} \mathrm{NMR}\left(100 \mathrm{MHz}, \mathrm{CDCl}_{3}\right) \delta$ (major + minor) $175.7,175.2,160.5,159.0,154.8,148.8,138.7,138.0,137.3,133.2,126.8,126.4,126.2$, 125.0, 124.9, 124.0, 117.5, 107.9, 98.4, 97.7, 91.2, 90.7, 48.5, 44.6, 41.0, 39.4, 37.6, 36.7, 36.6, 36.0, 29.2, 27.7, 27.2, 23.4, 22.1. HRMS (ESI-TOF): calcd. for $\mathrm{C}_{24} \mathrm{H}_{20} \mathrm{NO}_{5}[\mathrm{M}+\mathrm{H}]^{+} 402.1336$; found 402.1346 .

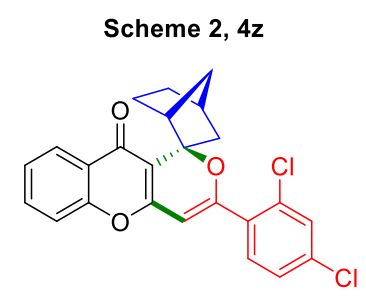

Compound 4z: purification by flash column chromatography on silica gel (eluent: petroleum ether/ethyl acetate/dichloromethane $=$ 20:1:0.1 - 3:1:0.1); light yellow solid, $139.5 \mathrm{mg}, 82 \%$ yield, mp $160.8-162.5^{\circ} \mathrm{C} ; 90: 10 \mathrm{dr} ;{ }^{1} \mathrm{H}$ NMR $\left(400 \mathrm{MHz}, \mathrm{CDCl}_{3}\right) \delta$ (major + minor) $8.18(\mathrm{~d}, J=7.6 \mathrm{~Hz}$, 1H), $7.71(\mathrm{~d}, J=8.4 \mathrm{~Hz}, 0.10 \mathrm{H}), 7.63-7.56(\mathrm{~m}, 2 \mathrm{H}), 7.46(\mathrm{~s}, 1 \mathrm{H}), 7.38-7.30(\mathrm{~m}, 3 \mathrm{H}), 6.54(\mathrm{~s}$, 0.10H), 6.37 (s, 0.90H), $3.77(\mathrm{~d}, J=13.4 \mathrm{~Hz}, 1 \mathrm{H}), 2.95$ (s, 0.90H), $2.86(\mathrm{~s}, 0.10 \mathrm{H}), 2.46(\mathrm{~s}, 1 \mathrm{H})$, $2.15(\mathrm{~d}, J=10.4 \mathrm{~Hz}, 0.10 \mathrm{H}), 1.88(\mathrm{~d}, J=9.6 \mathrm{~Hz}, 0.90 \mathrm{H}), 1.82-1.70(\mathrm{~m}, 2 \mathrm{H}), 1.51-1.43(\mathrm{~m}, 1 \mathrm{H})$, $1.30-1.19(\mathrm{~m}, 3 \mathrm{H}) ;{ }^{13} \mathrm{C} \mathrm{NMR}\left(100 \mathrm{MHz}, \mathrm{CDCl}_{3}\right) \delta$ (major + minor) $175.8,160.7,158.8,154.8$, 136.4, 133.3, 133.0, 132.9, 131.2, 131.0, 130.7, 129.9, 127.6, 127.4, 126.1, 125.1, 124.8, 117.5, 
102.0, 101.0, 91.2, 48.2, 44.7, 41.1, 39.4, 37.6, 36.8, 36.5, 36.2, 29.2, 27.8, 23.6, 22.2. HRMS (ESI-TOF): calcd. for $\mathrm{C}_{24} \mathrm{H}_{19} \mathrm{Cl}_{2} \mathrm{O}_{3}[\mathrm{M}+\mathrm{H}]^{+} 425.0706$; found 425.0705 .

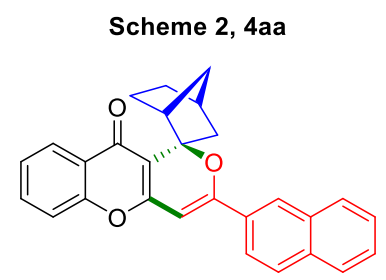

Compound 4aa: purification by flash column chromatography on silica gel (eluent: petroleum ether/ethyl acetate/dichloromethane $=20: 1: 0.1-3: 1: 0.1)$; yellow solid, $130.1 \mathrm{mg}, 80 \%$ yield, $\mathrm{mp}$ $185.7-187.0{ }^{\circ} \mathrm{C} ; 87: 13 \mathrm{dr} ;{ }^{1} \mathrm{H}$ NMR $\left(400 \mathrm{MHz}, \mathrm{CDCl}_{3}\right) \delta$ (major + minor) 8.37 (s, 0.13H), 8.31 (s, $0.87 \mathrm{H}), 8.22(\mathrm{~d}, J=8.0 \mathrm{~Hz}, 1 \mathrm{H}), 7.96-7.92(\mathrm{~m}, 1 \mathrm{H}), 7.89-7.81(\mathrm{~m}, 3 \mathrm{H}), 7.62-7.54(\mathrm{~m}, 3 \mathrm{H})$, $7.41-7.34(\mathrm{~m}, 2 \mathrm{H}), 6.50-6.47(\mathrm{~m}, 0.13 \mathrm{H}), 6.44-6.42(\mathrm{~m}, 0.87 \mathrm{H}), 3.98(\mathrm{~d}, J=13.6 \mathrm{~Hz}, 0.13 \mathrm{H})$, $3.87(\mathrm{dd}, J=13.6,2.6 \mathrm{~Hz}, 0.87 \mathrm{H}), 2.90(\mathrm{~s}, 0.87 \mathrm{H}), 2.81(\mathrm{~s}, 0.13 \mathrm{H}), 2.56(\mathrm{~s}, 0.87 \mathrm{H}), 2.52(\mathrm{~s}$, $0.13 \mathrm{H}), 2.22-2.19(\mathrm{~m}, 0.13 \mathrm{H}), 2.09-2.04(\mathrm{~m}, 0.87 \mathrm{H}), 1.95-1.90(\mathrm{~m}, 0.87 \mathrm{H}), 1.87-1.81(\mathrm{~m}$, $0.87 \mathrm{H}), 1.69-1.63(\mathrm{~m}, 0.39 \mathrm{H}), 1.55-1.48(\mathrm{~m}, 0.87 \mathrm{H}), 1.32-1.21(\mathrm{~m}, 3 \mathrm{H}) ;{ }^{13} \mathrm{C} \mathrm{NMR}(100 \mathrm{MHz}$, $\left.\mathrm{CDCl}_{3}\right) \delta$ (major + minor) 175.7, 162.1, 161.8, 154.9, 134.6, 133.1, 132.9, 130.1, 129.2, 128.6, $128.5,127.9,127.7,126.9,126.7,126.3,126.2,125.2,124.7,122.9,122.6,117.5,107.0,95.7$, 95.1, 90.8, 48.5, 44.4, 39.4, 37.8, 36.8, 36.7, 36.1, 29.4, 28.0, 23.2, 22.3. HRMS (ESI-TOF): calcd. for $\mathrm{C}_{28} \mathrm{H}_{23} \mathrm{O}_{3}[\mathrm{M}+\mathrm{H}]^{+}$407.1642; found 407.1642.

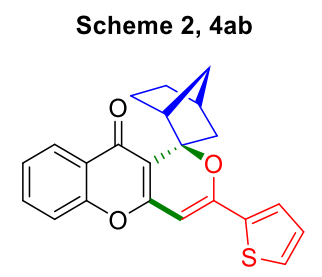

Compound 4ab: purification by flash column chromatography on silica gel (eluent: petroleum ether/ethyl acetate $=20: 1-3: 1)$; yellow solid, $97.1 \mathrm{mg}, 67 \%$ yield, $\mathrm{mp} 151.0-152.6{ }^{\circ} \mathrm{C} ; 80: 20 \mathrm{dr}$; ${ }^{1} \mathrm{H} \mathrm{NMR}\left(400 \mathrm{MHz}, \mathrm{CDCl}_{3}\right) \delta$ (major + minor) $8.19(\mathrm{~d}, J=7.6 \mathrm{~Hz}, 1 \mathrm{H}), 7.59-7.53(\mathrm{~m}, 2 \mathrm{H}), 7.49$ $-7.46(\mathrm{~m}, 1 \mathrm{H}), 7.39-7.32(\mathrm{~m}, 2 \mathrm{H}), 7.14-7.10(\mathrm{~m}, 1 \mathrm{H}), 6.17$ (s, 0.20H), $6.16(\mathrm{~s}, 0.80 \mathrm{H}), 3.92-$ $3.87(\mathrm{~m}, 0.20 \mathrm{H}), 3.80(\mathrm{~d}, J=13.2 \mathrm{~Hz}, 0.80 \mathrm{H}), 2.88(\mathrm{~s}, 0.80 \mathrm{H}), 2.79(\mathrm{~s}, 0.20 \mathrm{H}), 2.58-2.57(\mathrm{~m}$, $0.20 \mathrm{H}), 2.51-2.48(\mathrm{~m}, 0.80 \mathrm{H}), 2.16(\mathrm{~d}, \mathrm{~J}=9.8 \mathrm{~Hz}, 0.20 \mathrm{H}), 1.97(\mathrm{~d}, J=9.8 \mathrm{~Hz}, 0.80 \mathrm{H}), 1.81-$ 
$1.77(\mathrm{~m}, 2 \mathrm{H}), 1.57-1.48(\mathrm{~m}, 1 \mathrm{H}), 1.30-1.16(\mathrm{~m}, 3 \mathrm{H}) ;{ }^{13} \mathrm{C} \mathrm{NMR}\left(100 \mathrm{MHz}, \mathrm{CDCl}_{3}\right) \delta$ (major + minor) $175.4,161.6,157.3,154.8,137.4,132.8,129.5,129.4,128.3,127.8,127.7,126.1,125.1$, 124.7, 117.4, 110.1, 107.0, 94.1, 93.6, 91.1, 90.5, 48.5, 44.3, 41.0, 39.3, 37.8, 36.7, 36.6, 35.8, 29.3, 28.0, 22.9, 22.3. HRMS (ESI-TOF): calcd. for $\mathrm{C}_{22} \mathrm{H}_{18} \mathrm{NaO}_{3} \mathrm{~S}[\mathrm{M}+\mathrm{Na}]^{+}$385.0869; found 385.0872.

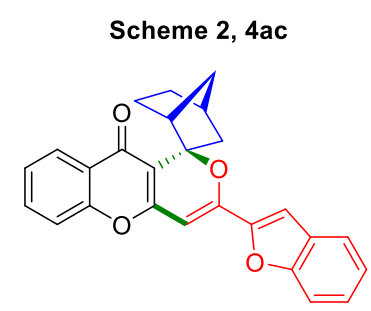

Compound 4ac: purification by flash column chromatography on silica gel (eluent: petroleum ether/ethyl acetate/dichloromethane $=20: 1: 0.1-3: 1: 0.1)$; yellow solid, $120.5 \mathrm{mg}, 76 \%$ yield, $\mathrm{mp}$ 196.0 - $197.3{ }^{\circ} \mathrm{C} ; 88: 12 \mathrm{dr} ;{ }^{1} \mathrm{H} \mathrm{NMR}\left(400 \mathrm{MHz}, \mathrm{CDCl}_{3}\right) \delta$ (major + minor) $8.19(\mathrm{~d}, J=8.0 \mathrm{~Hz}$, 1H), $7.74-7.68(\mathrm{~m}, 0.24 \mathrm{H}), 7.66-7.58(\mathrm{~m}, 1.76 \mathrm{H}), 7.51(\mathrm{dd}, J=8.4,1.0 \mathrm{~Hz}, 0.88 \mathrm{H}), 7.48-7.46$ (m, 0.12H), $7.40-7.33(\mathrm{~m}, 3 \mathrm{H}), 7.29-7.25(\mathrm{~m}, 1 \mathrm{H}), 7.20(\mathrm{~s}, 1 \mathrm{H}), 6.49(\mathrm{~s}, 0.12 \mathrm{H}), 6.47$ (s, $0.88 \mathrm{H}), 3.98-3.93(\mathrm{~m}, 0.12 \mathrm{H}), 3.80(\mathrm{dd}, J=13.6,2.4 \mathrm{~Hz}, 0.88 \mathrm{H}), 2.89(\mathrm{~d}, J=4.4 \mathrm{~Hz}, 0.88 \mathrm{H})$, $2.78(\mathrm{~d}, J=3.8 \mathrm{~Hz}, 0.12 \mathrm{H}), 2.62-2.61(\mathrm{~m}, 0.12 \mathrm{H}), 2.52-2.50(\mathrm{~m}, 0.88 \mathrm{H}), 2.17(\mathrm{~d}, J=10.2 \mathrm{~Hz}$, $0.12 \mathrm{H}), 1.94(\mathrm{~d}, J=9.2 \mathrm{~Hz}, 0.88 \mathrm{H}), 1.82-1.76(\mathrm{~m}, 1.76 \mathrm{H}), 1.70-1.68(\mathrm{~m}, 0.12 \mathrm{H}), 1.56-1.52$ $(\mathrm{m}, 0.24 \mathrm{H}), 1.50-1.46(\mathrm{~m}, 0.88 \mathrm{H}), 1.31-1.16(\mathrm{~m}, 3 \mathrm{H}) ;{ }^{13} \mathrm{C} \mathrm{NMR}\left(100 \mathrm{MHz}, \mathrm{CDCl}_{3}\right) \delta$ (major + minor) $175.7,160.9,160.7,155.7,154.9,153.8,153.2,149.8,132.97,133.0,128.1,126.7,126.6$, 126.1, 125.1, 124.8, 123.7, 122.3, 122.2, 117.5, 111.6, 110.1, 108.4, 108.3, 108.1, 97.0, 96.3, 90.8, 90.2, 48.9, 44.3, 40.7, 39.3, 37.6, 36.7, 35.7, 29.4, 28.0, 22.6, 22.2. HRMS (ESI-TOF): calcd. for $\mathrm{C}_{26} \mathrm{H}_{21} \mathrm{O}_{4}[\mathrm{M}+\mathrm{H}]^{+}$397.1434; found 397.1436.

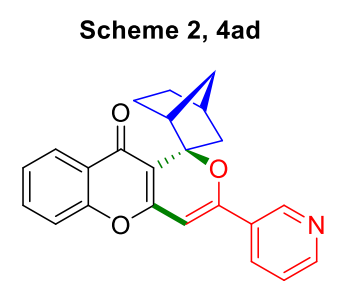

Compound 4ad: purification by flash column chromatography on silica gel (eluent: petroleum ether/ethyl acetate/dichloromethane $=18: 1: 0.1-3: 1: 0.1) ;$ yellow solid, $37.7 \mathrm{mg}, 26 \%$ yield, $\mathrm{mp}$ S19 
$182.9-184.1{ }^{\circ} \mathrm{C} ; 87: 13 \mathrm{dr} ;{ }^{1} \mathrm{H}$ NMR $\left(400 \mathrm{MHz}, \mathrm{CDCl}_{3}\right) \delta$ (major + minor) 9.07 (s, $0.13 \mathrm{H}$ ), 9.02 $(\mathrm{s}, 0.87 \mathrm{H}), 8.67(\mathrm{~d}, J=4.8 \mathrm{~Hz}, 1 \mathrm{H}), 8.18(\mathrm{dd}, J=8.0,1.8 \mathrm{~Hz}, 1 \mathrm{H}), 8.08(\mathrm{~d}, J=8.4 \mathrm{~Hz}, 0.13 \mathrm{H})$, $8.04(\mathrm{~d}, J=8.0 \mathrm{~Hz}, 0.87 \mathrm{H}), 7.61-7.56(\mathrm{~m}, 1 \mathrm{H}), 7.40-7.31(\mathrm{~m}, 3 \mathrm{H}), 6.38(\mathrm{~s}, 0.13 \mathrm{H}), 6.33(\mathrm{~s}$, 0.87H), $3.91-3.86(\mathrm{~m}, 0.13 \mathrm{H}), 3.80(\mathrm{dd}, J=13.4,2.4 \mathrm{~Hz}, 0.87 \mathrm{H}), 2.85(\mathrm{~s}, 0.87 \mathrm{H}), 2.76(\mathrm{~s}$, $0.13 \mathrm{H}), 2.50(\mathrm{~s}, 1 \mathrm{H}), 2.16(\mathrm{~d}, J=10.4 \mathrm{~Hz}, 0.13 \mathrm{H}), 1.91(\mathrm{~d}, J=9.2 \mathrm{~Hz}, 0.87 \mathrm{H}), 1.84-1.75(\mathrm{~m}$, $2 \mathrm{H}), 1.51-1.46(\mathrm{~m}, 1 \mathrm{H}), 1.27-1.18(\mathrm{~m}, 3 \mathrm{H}) ;{ }^{13} \mathrm{C} \mathrm{NMR}\left(100 \mathrm{MHz}, \mathrm{CDCl}_{3}\right) \delta$ (major + minor) $175.7,160.9,159.7,159.4,154.8,151.4,151.3,147.5,147.3,133.5,133.0,133.0,129.0,126.2$, 125.1, 124.8, 123.6, 117.5, 107.4, 96.3, 95.8, 91.1, 48.5, 44.5, 41.1, 39.4, 37.7, 36.7, 36.6, 36.0, 29.2, 27.8, 23.3, 22.2. HRMS (ESI-TOF): calcd. for $\mathrm{C}_{23} \mathrm{H}_{20} \mathrm{NO}_{3}[\mathrm{M}+\mathrm{H}]^{+}$358.1438; found 358.1435 .

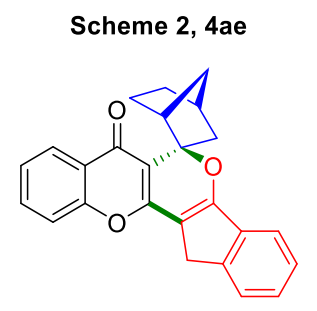

Compound 4ae: purification by flash column chromatography on silica gel (eluent: petroleum ether/ethyl acetate $=20: 1-4: 1$ ); yellow solid, $66.3 \mathrm{mg}, 45 \%$ yield, $\mathrm{mp} 182.8-184.2{ }^{\circ} \mathrm{C} ; 91: 9 \mathrm{dr}$; ${ }^{1} \mathrm{H} \mathrm{NMR}\left(400 \mathrm{MHz}, \mathrm{CDCl}_{3}\right) \delta$ (major + minor) $8.20(\mathrm{dd}, J=8.0,1.8 \mathrm{~Hz}, 1 \mathrm{H}), 7.60-7.52(\mathrm{~m}, 3 \mathrm{H})$, $7.41-7.31(\mathrm{~m}, 4 \mathrm{H}), 3.95(\mathrm{dd}, J=13.6,2.4 \mathrm{~Hz}, 1 \mathrm{H}), 3.82-3.65(\mathrm{~m}, 2 \mathrm{H}), 2.85(\mathrm{~s}, 0.91 \mathrm{H}), 2.74(\mathrm{~s}$, $0.09 \mathrm{H}), 2.55(\mathrm{~s}, 0.09 \mathrm{H}), 2.50(\mathrm{~s}, 0.91 \mathrm{H}), 2.16(\mathrm{~d}, J=10.2 \mathrm{~Hz}, 0.09 \mathrm{H}), 1.99(\mathrm{~d}, J=10.2 \mathrm{~Hz}, 0.91 \mathrm{H})$, $1.87-1.76(\mathrm{~m}, 1.82 \mathrm{H}), 1.70-1.69(\mathrm{~m}, 0.09 \mathrm{H}), 1.58-1.54(\mathrm{~m}, 0.18 \mathrm{H}), 1.52-1.44(\mathrm{~m}, 0.91 \mathrm{H})$, $1.251 .22(\mathrm{~m}, 2 \mathrm{H}), 1.13(\mathrm{dd}, J=9.6,2.2 \mathrm{~Hz}, 1 \mathrm{H}) ;{ }^{13} \mathrm{C} \mathrm{NMR}\left(100 \mathrm{MHz}, \mathrm{CDCl}_{3}\right) \delta$ (major + minor) 175.2, 165.1, 160.5, 154.4, 144.8, 136.7, 132.6, 129.1, 128.9, 127.3, 127.2, 126.2, 125.4, 125.0, 125.0, 124.7, 120.1, 117.3, 108.6, 105.1, 93.9, 49.0, 45.2, 40.7, 39.3, 37.5, 36.8, 35.7, 31.5, 29.5, 28.1, 23.2, 22.3. HRMS (ESI-TOF): calcd. for $\mathrm{C}_{25} \mathrm{H}_{21} \mathrm{O}_{3}[\mathrm{M}+\mathrm{H}]^{+}$369.1485; found 369.1482. 


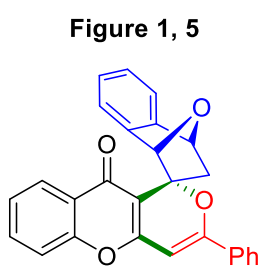

Compound 5: purification by flash column chromatography on silica gel (eluent: petroleum ether/ethyl acetate/dichloromethane $=18: 1: 0.1-3: 1: 0.1)$; yellow solid, $48.8 \mathrm{mg}, 30 \%$ yield, $\mathrm{mp}$ $221.0-222.8^{\circ} \mathrm{C} ; 99: 1 \mathrm{dr} ;{ }^{1} \mathrm{H}$ NMR $\left(400 \mathrm{MHz}, \mathrm{CDCl}_{3}\right) \delta 7.93-7.87(\mathrm{~m}, 2 \mathrm{H}), 7.76(\mathrm{~d}, J=8.0 \mathrm{~Hz}$, $1 \mathrm{H}), 7.55(\mathrm{t}, J=8.0 \mathrm{~Hz}, 1 \mathrm{H}), 7.50-7.48(\mathrm{~m}, 3 \mathrm{H}), 7.37(\mathrm{dd}, J=10.8,7.8 \mathrm{~Hz}, 2 \mathrm{H}), 7.27-7.21(\mathrm{~m}$, 2H), $6.89(\mathrm{t}, J=7.4 \mathrm{~Hz}, 1 \mathrm{H}), 6.83(\mathrm{~d}, J=7.2 \mathrm{~Hz}, 1 \mathrm{H}), 6.38(\mathrm{~s}, 1 \mathrm{H}), 5.68(\mathrm{~d}, J=5.0 \mathrm{~Hz}, 1 \mathrm{H}), 5.48$ $(\mathrm{s}, 1 \mathrm{H}), 4.11(\mathrm{~d}, J=12.2 \mathrm{~Hz}, 1 \mathrm{H}), 2.45(\mathrm{dd}, J=12.2,5.2 \mathrm{~Hz}, 1 \mathrm{H}) ;{ }^{13} \mathrm{C} \mathrm{NMR}\left(100 \mathrm{MHz}, \mathrm{CDCl}_{3}\right) \delta$ $172.9,162.8,160.9,154.7,147.5,139.9,132.8,132.5,131.5,128.9,128.0,126.8,126.5,125.9$, 124.7, 124.5, 122.0, 119.3, 117.4, 104.7, 94.8, 87.8, 82.2, 80.0, 38.3. HRMS (ESI-TOF): calcd. for $\mathrm{C}_{27} \mathrm{H}_{19} \mathrm{O}_{4}[\mathrm{M}+\mathrm{H}]^{+}$407.1278; found 407.1281.

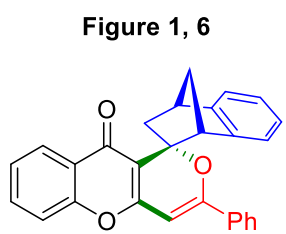

Compound 6: purification by flash column chromatography on silica gel (eluent: petroleum ether/ethyl acetate/dichloromethane $=20: 1: 0.1-3: 1: 0.1) ;$ yellow solid, $50.2 \mathrm{mg}, 31 \%$ yield, $\mathrm{mp}$ $158.9-160.6{ }^{\circ} \mathrm{C} ; 99: 1 \mathrm{dr} ;{ }^{1} \mathrm{H}$ NMR $\left(400 \mathrm{MHz}, \mathrm{CDCl}_{3}\right) \delta 8.23(\mathrm{~d}, J=8.0 \mathrm{~Hz}, 1 \mathrm{H}), 7.61(\mathrm{ddt}, J=$ 8.4, 7.2, 1.6 Hz, 1H), $7.43-7.31(\mathrm{~m}, 4 \mathrm{H}), 7.24(\mathrm{td}, J=7.8,1.6 \mathrm{~Hz}, 2 \mathrm{H}), 7.19-7.14(\mathrm{~m}, 1 \mathrm{H}), 7.12$ $(\mathrm{dd}, J=7.2,1.6 \mathrm{~Hz}, 2 \mathrm{H}), 6.92(\mathrm{tt}, J=7.4,1.2 \mathrm{~Hz}, 1 \mathrm{H}), 6.82(\mathrm{~d}, J=7.2 \mathrm{~Hz}, 1 \mathrm{H}), 6.42(\mathrm{~d}, J=1.4$ $\mathrm{Hz}, 1 \mathrm{H}), 4.42-4.37(\mathrm{~m}, 1 \mathrm{H}), 3.84(\mathrm{~s}, 1 \mathrm{H}), 3.55(\mathrm{~d}, J=3.4 \mathrm{~Hz}, 1 \mathrm{H}), 2.51(\mathrm{dd}, J=9.6,1.4 \mathrm{~Hz}, 1 \mathrm{H})$, $1.87-1.82(\mathrm{~m}, 1 \mathrm{H}), 1.57(\mathrm{ddd}, J=12.8,3.6,1.4 \mathrm{~Hz}, 1 \mathrm{H}) ;{ }^{13} \mathrm{C} \mathrm{NMR}\left(100 \mathrm{MHz}, \mathrm{CDCl}_{3}\right) \delta 174.9$, $162.5,162.3,154.8,149.5,143.6,132.9,131.4,131.2,128.2,126.8,126.4,126.2,125.7,125.1$, 124.8, 124.3, 120.6, 117.5, 109.6, 94.4, 89.0, 55.8, 48.4, 43.8, 38.5; HRMS (ESI-TOF): calcd. for $\mathrm{C}_{28} \mathrm{H}_{21} \mathrm{O}_{3}[\mathrm{M}+\mathrm{H}]^{+}$405.1485; found 405.1489. 


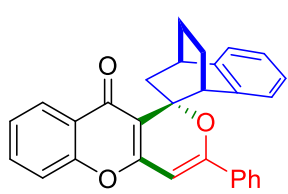

Compound 7: purification by flash column chromatography on silica gel (eluent: petroleum ether/ethyl acetate/dichloromethane $=$ 20:1:0.1 $-3: 1: 0.1$; yellow solid, $58.6 \mathrm{mg}, 35 \%$ yield, $\mathrm{mp}$ 169.0-170.8 ${ }^{\circ} \mathrm{C}$; 99:1 dr; ${ }^{1} \mathrm{H}$ NMR $\left(400 \mathrm{MHz}, \mathrm{CDCl}_{3}\right) \delta 8.25(\mathrm{~d}, J=8.0 \mathrm{~Hz}, 1 \mathrm{H}), 7.64-7.58(\mathrm{~m}$, 1H), $7.42-7.33(\mathrm{~m}, 3 \mathrm{H}), 7.31-7.27(\mathrm{~m}, 2 \mathrm{H}), 7.21(\mathrm{t}, J=7.6 \mathrm{~Hz}, 2 \mathrm{H}), 7.05(\mathrm{~d}, J=7.8 \mathrm{~Hz}, 3 \mathrm{H})$, $6.83(\mathrm{~d}, J=7.2 \mathrm{~Hz}, 1 \mathrm{H}), 6.34(\mathrm{~s}, 1 \mathrm{H}), 4.31(\mathrm{dd}, J=14.2,2.4 \mathrm{~Hz}, 1 \mathrm{H}), 3.69(\mathrm{~s}, 1 \mathrm{H}), 3.34(\mathrm{~s}, 1 \mathrm{H})$, $2.38(\mathrm{t}, J=10.8 \mathrm{~Hz}, 1 \mathrm{H}), 1.83(\mathrm{dt}, J=13.2,6.8 \mathrm{~Hz}, 1 \mathrm{H}), 1.72-1.64(\mathrm{~m}, 1 \mathrm{H}), 1.40-1.31(\mathrm{~m}, 1 \mathrm{H})$, $1.30-1.21(\mathrm{~m}, 1 \mathrm{H}) ;{ }^{13} \mathrm{C} \mathrm{NMR}\left(100 \mathrm{MHz}, \mathrm{CDCl}_{3}\right) \delta 175.6,162.2,161.3,154.8,143.9,139.6$, 133.0, 131.7, 131.1, 128.2, 126.9, 126.7, 126.3, 126.1, 125.6, 125.3, 124.8, 123.4, 117.5, 108.6, 93.7, 85.7, 42.4, 36.3, 35.5, 25.1, 20.1; HRMS (ESI-TOF): calcd. for $\mathrm{C}_{29} \mathrm{H}_{23} \mathrm{O}_{3}[\mathrm{M}+\mathrm{H}]^{+} 419.1642$; found 419.1660

\section{Preparative-scale experiment of $4 a$ (Scheme 2).}

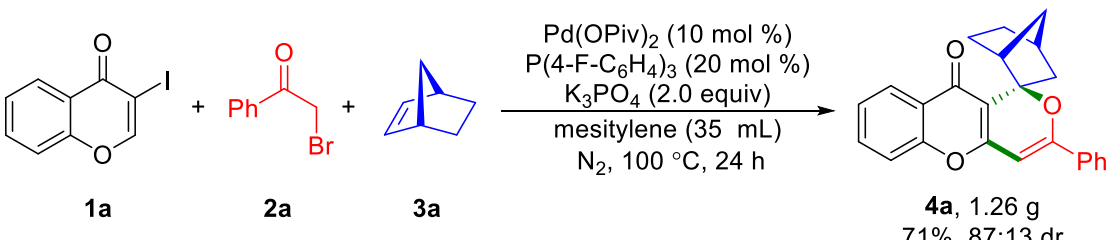

A $100 \mathrm{~mL}$ flame-dried flask with a stir bar was charged with 3-iodochromone (1a, $1.36 \mathrm{~g}, 5.0$ mmol), $\alpha$-bromoacetophenone (2a, $1.00 \mathrm{~g}, 5.0 \mathrm{mmol}$ ), NBE (3a, $1.88 \mathrm{~g}, 20.0 \mathrm{mmol}$ ), Pd(OPiv) 2 (154.3 mg, $0.5 \mathrm{mmol}), \mathrm{P}-\left(4-\mathrm{F}-\mathrm{C}_{6} \mathrm{H}_{4}\right)_{3}(316.3 \mathrm{mg}, 1.0 \mathrm{mmol})$, and $\mathrm{K}_{3} \mathrm{PO}_{4}(2.12 \mathrm{~g}, 10.0 \mathrm{mmol})$ in 32 $\mathrm{mL}$ of dry mesitylene under nitrogen atmosphere at $100{ }^{\circ} \mathrm{C}$ for $24 \mathrm{~h}$. After the completion of the reaction detected by thin layer chromatography (TLC), the mixture was cooled to room temperature and purified by flash column chromatography on silica gel (petroleum ether/ethyl acetate/dichloromethane $=20: 1: 0.1-3: 1: 0.1)$ to afford the desired product 4a as a yellow solid (1.26 g, 71\%, 87:13 dr). 


\section{Transformation of $4 a$ into 8 (Scheme 3a).}

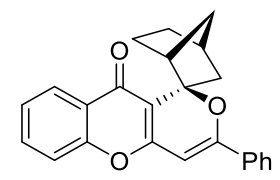

4a, $87: 13 \mathrm{dr}$

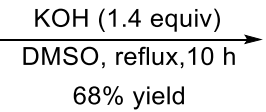

$68 \%$ yield

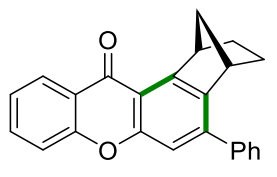

In a solution of $\mathbf{4 a}(106.9 \mathrm{mg}, 0.3 \mathrm{mmol})$ in DMSO $(3 \mathrm{~mL})$, an aqueous solution of $12 \mathrm{~N} \mathrm{KOH}$ $(35 \mu \mathrm{L})$ was added and the mixture was heated under reflux for $10 \mathrm{~h}$. After the completion of the reaction detected by thin layer chromatography (TLC), the mixture was cooled to room temperature and slowly acidified with $10 \mathrm{~N} \mathrm{HCl}$ to $\mathrm{pH}=3$ at $0{ }^{\circ} \mathrm{C}$. Afterwards, the mixture was extracted with ethyl acetate $(10 \mathrm{~mL} \times 5)$ and the combined organic extracts were washed with brine $(5 \mathrm{~mL} \times 5)$. The organic extracts were dried over anhydrous $\mathrm{Na}_{2} \mathrm{SO}_{4}$, filtered and concentrated by rotary evaporation. The residue was purified by flash column chromatography on silica gel (petroleum ether/ethyl acetate $=20: 1)$ to afford the desired product $\mathbf{8}(69.0 \mathrm{mg}, 68 \%$ yield). White solid, mp $119.0-120.7{ }^{\circ} \mathrm{C} ;{ }^{1} \mathrm{H}$ NMR $\left(400 \mathrm{MHz}, \mathrm{CDCl}_{3}\right) \delta 8.33(\mathrm{dd}, J=8.0,1.6 \mathrm{~Hz}, 1 \mathrm{H}), 7.71-$ $7.65(\mathrm{~m}, 1 \mathrm{H}), 7.55-7.49(\mathrm{~m}, 4 \mathrm{H}), 7.46-7.40(\mathrm{~m}, 2 \mathrm{H}), 7.34(\mathrm{t}, J=7.6 \mathrm{~Hz}, 1 \mathrm{H}), 7.28(\mathrm{~s}, 1 \mathrm{H}), 4.88$ $(\mathrm{d}, J=3.4 \mathrm{~Hz}, 1 \mathrm{H}), 3.59(\mathrm{~d}, J=2.8 \mathrm{~Hz}, 1 \mathrm{H}), 2.17-2.09(\mathrm{~m}, 1 \mathrm{H}), 2.06-1.99(\mathrm{~m}, 1 \mathrm{H}), 1.81(\mathrm{~d}, J=$ $9.0 \mathrm{~Hz}, 1 \mathrm{H}), 1.58(\mathrm{~d}, J=8.8 \mathrm{~Hz}, 1 \mathrm{H}), 1.34-1.26(\mathrm{~m}, 2 \mathrm{H}) ;{ }^{13} \mathrm{C} \mathrm{NMR}\left(100 \mathrm{MHz}, \mathrm{CDCl}_{3}\right) \delta 178.6$, $156.2,155.0,150.4,142.5,141.5,139.4,134.5,128.9,128.7,128.1,126.7,123.5,122.6,117.8$, 115.5, 114.5, 49.9, 43.7, 41.6, 26.7, 26.0; HRMS (ESI-TOF): calcd. for $\mathrm{C}_{24} \mathrm{H}_{19} \mathrm{O}_{2}[\mathrm{M}+\mathrm{H}]^{+}$ 339.1380; found 339.1579.

\section{Transformation of $4 \mathrm{f}$ into 9 (Scheme $3 \mathrm{~b}$ ).}

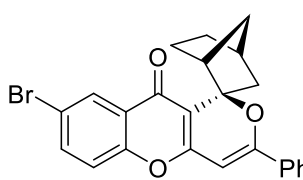

4f, $90: 10 \mathrm{dr}$

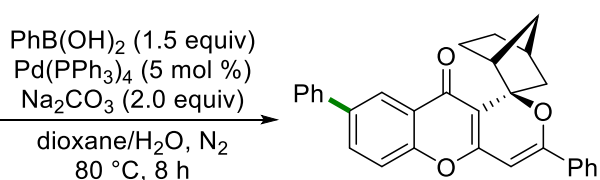

9

A $4 \mathrm{~mL}$ flame-dried vial with a stir bar was charged with $4 \mathbf{f}(87.1 \mathrm{mg}, 0.2 \mathrm{mmol})$, phenylboronic acid (36.6 mg, $0.3 \mathrm{mmol}), \mathrm{Pd}\left(\mathrm{PPh}_{3}\right)_{4}(0.01 \mathrm{mmol}, 11.6 \mathrm{mg})$, and $\mathrm{Na}_{2} \mathrm{CO}_{3}(42.4 \mathrm{mg}$, $0.4 \mathrm{mmol}$ ) in $2.0 \mathrm{~mL}$ deoxygenated 1,4-dioxane and $0.4 \mathrm{~mL}$ deoxygenated water under nitrogen atmosphere at $80{ }^{\circ} \mathrm{C}$ for $8 \mathrm{~h}$. After the completion of the reaction detected by thin layer 
chromatography (TLC), water $(10 \mathrm{~mL})$ was added to the mixture. Afterwards, the mixture was extracted with ethyl acetate $(10 \mathrm{~mL} \times 5)$, and the combined organic extracts were dried over anhydrous $\mathrm{Na}_{2} \mathrm{SO}_{4}$, filtered and concentrated by rotary evaporation. The residue was purified by flash column chromatography on silica gel (petroleum ether/ethyl acetate $=20: 1$ ) to afford the desired product 9 (62.3 mg, $72 \%$ yield). Yellow solid, mp $152.7-154.5^{\circ} \mathrm{C} ; 86: 14 \mathrm{dr} ;{ }^{1} \mathrm{H}$ NMR $\left(400 \mathrm{MHz}, \mathrm{CDCl}_{3}\right) \delta 8.46(\mathrm{~d}, J=2.4 \mathrm{~Hz}, 1 \mathrm{H}), 7.88-7.77(\mathrm{~m}, 3 \mathrm{H}), 7.71-7.64(\mathrm{~m}, 2 \mathrm{H}), 7.47-$ $7.43(\mathrm{~m}, 6 \mathrm{H}), 7.36(\mathrm{t}, J=7.2 \mathrm{~Hz}, 1 \mathrm{H}), 6.37(\mathrm{~s}, 0.14 \mathrm{H}), 6.31(\mathrm{~s}, 0.86 \mathrm{H}), 3.95(\mathrm{dt}, J=10.6,2.4 \mathrm{~Hz}$, $0.14 \mathrm{H}), 3.86(\mathrm{dd}, J=13.6,2.4 \mathrm{~Hz}, 0.86 \mathrm{H}), 2.89(\mathrm{~s}, 0.86 \mathrm{H}), 2.81(\mathrm{~d}, J=3.8 \mathrm{~Hz}, 0.14 \mathrm{H}), 2.54-$ $2.50(\mathrm{~m}, 1 \mathrm{H}), 2.21(\mathrm{~d}, J=10.4 \mathrm{~Hz}, 0.14 \mathrm{H}), 2.00(\mathrm{~d}, J=9.8 \mathrm{~Hz}, 0.86 \mathrm{H}), 1.90-1.80(\mathrm{~m}, 2 \mathrm{H}), 1.62$ $(\mathrm{dd}, J=13.2,3.4 \mathrm{~Hz}, 0.14 \mathrm{H}), 1.57-1.47(\mathrm{~m}, 0.86 \mathrm{H}), 1.33-1.19(\mathrm{~m}, 3 \mathrm{H}) ;{ }^{13} \mathrm{C} \mathrm{NMR}(100 \mathrm{MHz}$, $\left.\mathrm{CDCl}_{3}\right) \delta 175.6,162.3,161.9,154.2,139.7,137.7,132.9,131.6,131.2,131.1,129.0,128.9,128.8$, $128.7,127.7,127.2,127.1,126.3,126.0,125.2,124.1,117.9,106.8,95.0,94.5,90.8,90.2,48.5$, 44.4, 41.1, 39.5, 37.8, 36.8, 36.6, 36.0, 29.3, 28.0, 23.2, 22.3; HRMS (ESI-TOF): calcd. for $\mathrm{C}_{30} \mathrm{H}_{25} \mathrm{O}_{3}[\mathrm{M}+\mathrm{H}]^{+}$433.1798; found 433.1795.

\section{Procedure for the synthesis of compound 4a' (Scheme 4a).}

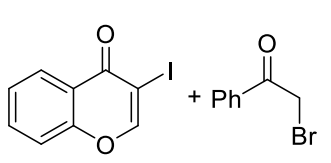

$1 \mathrm{a}$

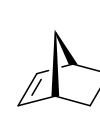

$3 \mathbf{a}$

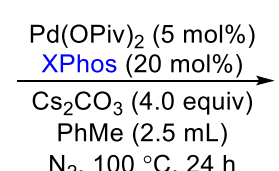

$\mathrm{N}_{2}, 100^{\circ} \mathrm{C}, 24 \mathrm{~h}$

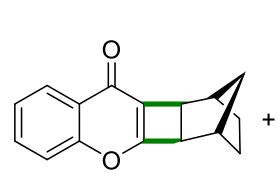

$4 a^{\prime}, 12 \%$

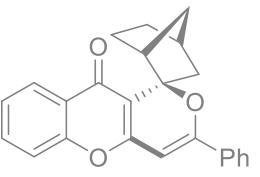

$4 a, 5 \%$

A $4 \mathrm{~mL}$ flame-dried vial with a stir bar was charged with 3-iodochromone (1a, $108.8 \mathrm{mg}, 0.4$ mmol), $\alpha$-bromoacetophenone (2a, $79.6 \mathrm{mg}, 0.4 \mathrm{mmol}$ ), norbornene (3a, $150.6 \mathrm{mg}, 1.6 \mathrm{mmol}$ ), $\mathrm{Pd}(\mathrm{OPiv})_{2}(6.2 \mathrm{mg}, 0.02 \mathrm{mmol})$, XPhos (38.1 mg, $\left.0.08 \mathrm{mmol}\right)$, and $\mathrm{Cs}_{2} \mathrm{CO}_{3}(521.3 \mathrm{mg}, 1.6 \mathrm{mmol})$ in $2.5 \mathrm{~mL}$ of dry toluene under nitrogen atmosphere at $100{ }^{\circ} \mathrm{C}$ for $24 \mathrm{~h}$. After the completion of the reaction detected by thin layer chromatography (TLC), the mixture was cooled to room temperature and purified by flash column chromatography on silica gel (petroleum ether/ethyl acetate $=20: 1-3: 1)$ to afford compound $\mathbf{4} \mathbf{a}^{\prime}$ as a white solid $(11.4 \mathrm{mg}, 12 \%)$ and $\mathbf{4} \mathbf{a}$ as a yellow solid (7.1 mg, 5\%). Compound 4a', mp $152.3-154.1{ }^{\circ} \mathrm{C} ;{ }^{1} \mathrm{H}$ NMR (400 MHz, $\left.\mathrm{CDCl}_{3}\right) \delta 8.23(\mathrm{dd}$, $J=8.0,1.7 \mathrm{~Hz}, 1 \mathrm{H}), 7.58(\mathrm{ddd}, J=8.6,7.1,1.7 \mathrm{~Hz}, 1 \mathrm{H}), 7.41(\mathrm{dd}, J=8.4,1.2 \mathrm{~Hz}, 1 \mathrm{H}), 7.36(\mathrm{ddd}, J=$ 8.2, 7.1, $1.2 \mathrm{~Hz}, 1 \mathrm{H}), 3.25$ (d, $J=3.2 \mathrm{~Hz}, 1 \mathrm{H}), 3.00(\mathrm{~d}, J=3.0 \mathrm{~Hz}, 1 \mathrm{H}), 2.50(\mathrm{~s}, 1 \mathrm{H}), 2.37$ (s, 1H), 1.66 
$-1.59(\mathrm{~m}, 2 \mathrm{H}), 1.25-1.09(\mathrm{~m}, 4 \mathrm{H}) ;{ }^{13} \mathrm{C} \mathrm{NMR}\left(100 \mathrm{MHz}, \mathrm{CDCl}_{3}\right) \delta 172.7,165.6,157.8,132.5$, 127.4, 126.5, 124.9, 122.5, 118.5, 54.3, 45.0, 35.3, 33.8, 31.5, 27.6, 27.5. HRMS (ESI-TOF): calcd. for $\mathrm{C}_{16} \mathrm{H}_{15} \mathrm{O}_{2}[\mathrm{M}+\mathrm{H}]^{+}$239.1067; found 239.1069.

\section{Procedure for the synthesis of compound $4 a-D$ (Scheme $4 b$ ).}
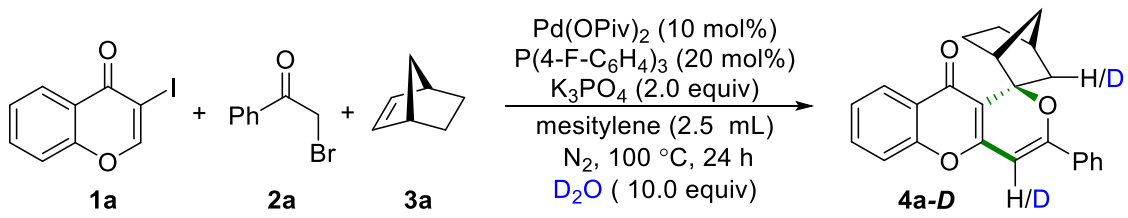

A $4 \mathrm{~mL}$ flame-dried vial with a stir bar was charged with 3-iodochromone (1a, $108.8 \mathrm{mg}, 0.4$ mmol), $\alpha$-bromoacetophenone (2a, $79.6 \mathrm{mg}, 0.4 \mathrm{mmol}$ ), norbornene (3a, $150.6 \mathrm{mg}, 1.6 \mathrm{mmol}$ ), $\mathrm{Pd}(\mathrm{OPiv})_{2}(12.3 \mathrm{mg}, 0.04 \mathrm{mmol}), \mathrm{P}-\left(4-\mathrm{F}-\mathrm{C}_{6} \mathrm{H}_{4}\right)_{3}(25.3 \mathrm{mg}, 0.08 \mathrm{mmol}), \mathrm{K}_{3} \mathrm{PO}_{4}(169.8 \mathrm{mg}, 0.8$ mmol), and $\mathrm{D}_{2} \mathrm{O}(72.5 \mu \mathrm{L}, 4.0 \mathrm{mmol})$ in $2.5 \mathrm{~mL}$ of dry mesitylene under nitrogen atmosphere at $100{ }^{\circ} \mathrm{C}$ for $24 \mathrm{~h}$. After the completion of the reaction detected by thin layer chromatography (TLC), the mixture was cooled to room temperature and purified by flash column chromatography on silica gel (petroleum ether/ethyl acetate/dichloromethane $=20: 1: 0.1-3: 1: 0.1$ ) to afford the desired product $\mathbf{4 a - D}$ as a yellow solid $(81.4 \mathrm{mg})$. 
10. X-ray crystal data for 4a, 4ac, 4ad, 4ae, 5-8, and 4a'.
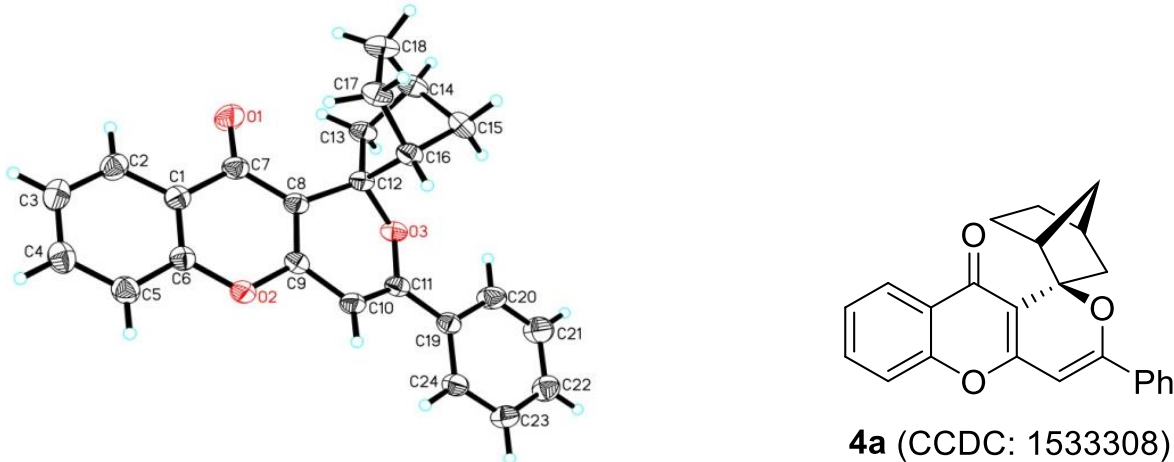

4a (CCDC: 1533308)

Identification code

$4 a$

Empirical formula

Formula weight

$\mathrm{C}_{24} \mathrm{H}_{20} \mathrm{O}_{3}$

Temperature/K

356.40

Crystal system

292(2)

Space group

monoclinic

$\mathrm{a} / \AA ̊$

$\mathrm{P} 2{ }_{1} / \mathrm{c}$

$\mathrm{b} / \AA$

18.7517(3)

c/Å

8.12380(10)

$\alpha /^{\circ}$

$12.3645(2)$

90

$\beta /{ }^{\circ}$

107.096(2)

$\gamma /{ }^{\circ}$

90

Volume/ $\AA^{3}$

1800.32(5)

Z

4

$\rho_{\text {calc }} \mathrm{g} / \mathrm{cm}^{3}$

1.315

$\mu / \mathrm{mm}^{-1}$

0.685

$\mathrm{F}(000)$

752.0

Crystal size $/ \mathrm{mm}^{3}$

$0.300 \times 0.240 \times 0.220$

Radiation

$\mathrm{CuK} \alpha(\lambda=1.54184)$

$2 \theta$ range for data collection/ ${ }^{\circ}$

9.87 to 142.49

Index ranges

$-22 \leq \mathrm{h} \leq 22,-9 \leq \mathrm{k} \leq 8,-13 \leq 1 \leq 15$

Reflections collected

6677

Independent reflections

$3405\left[R_{\text {int }}=0.0158, R_{\text {sigma }}=0.0164\right]$

$3405 / 0 / 245$

Goodness-of-fit on $\mathrm{F}^{2}$

1.036

Final R indexes $[\mathrm{I}>=2 \sigma(\mathrm{I})]$

$\mathrm{R}_{1}=0.0393, \mathrm{wR}_{2}=0.1083$

Final $R$ indexes [all data]

$\mathrm{R}_{1}=0.0428, \mathrm{wR}_{2}=0.1124$

Largest diff. peak/hole / e $\AA^{-3}$ 

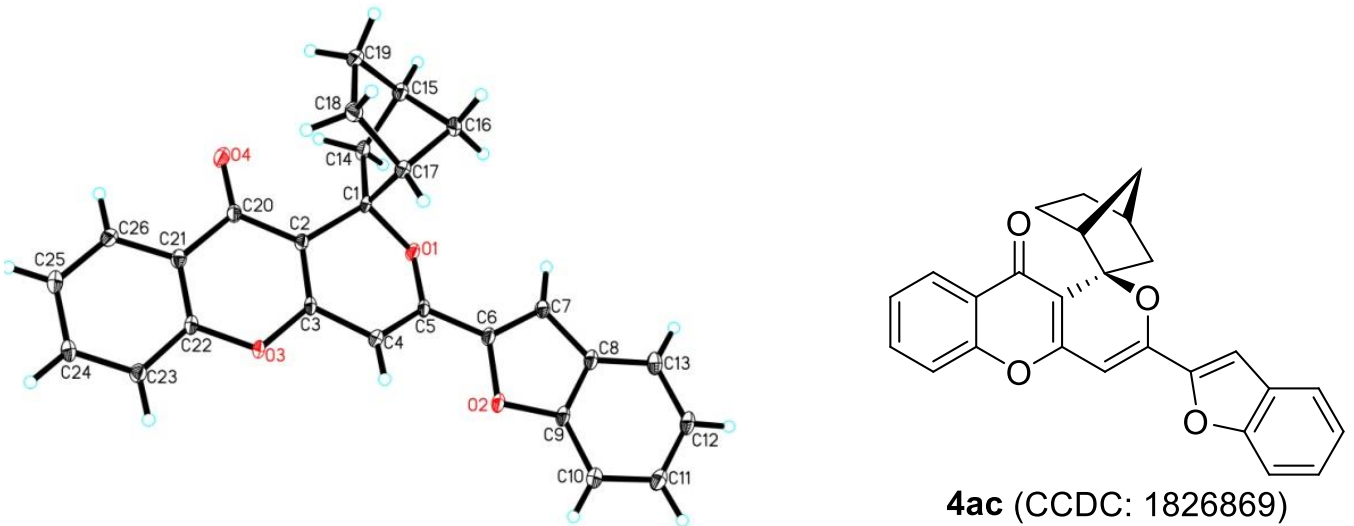

4ac (CCDC: 1826869)

\begin{tabular}{|c|c|}
\hline Identification code & $4 \mathbf{a c}$ \\
\hline Empirical formula & $\mathrm{C}_{26} \mathrm{H}_{20} \mathrm{O}_{4}$ \\
\hline Formula weight & 396.42 \\
\hline Temperature/K & $100.00(10)$ \\
\hline Crystal system & orthorhombic \\
\hline Space group & Fdd2 \\
\hline $\mathrm{a} / \AA$ & $40.148(4)$ \\
\hline $\mathrm{b} / \AA$ & $27.454(2)$ \\
\hline $\mathrm{c} / \AA$ & $6.8273(5)$ \\
\hline$\alpha /{ }^{\circ}$ & 90 \\
\hline$\beta /{ }^{\circ}$ & 90 \\
\hline$\gamma /{ }^{\circ}$ & 90 \\
\hline Volume $/ \AA^{3}$ & $7525.3(11)$ \\
\hline $\mathrm{Z}$ & 16 \\
\hline$\rho_{\text {calc }} \mathrm{g} / \mathrm{cm}^{3}$ & 1.400 \\
\hline$\mu / \mathrm{mm}^{-1}$ & 0.094 \\
\hline $\mathrm{F}(000)$ & 3328.0 \\
\hline Crystal size $/ \mathrm{mm}^{3}$ & $0.22 \times 0.18 \times 0.14$ \\
\hline Radiation & $\operatorname{MoK} \alpha(\lambda=0.71073)$ \\
\hline$\theta$ range for data collection ${ }^{\circ}$ & 3.387 to 29.502 \\
\hline Index ranges & $-51 \leq \mathrm{h} \leq 41,-36 \leq \mathrm{k} \leq 26,-8 \leq 1 \leq 9$ \\
\hline Reflections collected & 8775 \\
\hline Independent reflections & $3996\left[\mathrm{R}_{\mathrm{int}}=0.0638, \mathrm{R}_{\text {sigma }}=0.0750\right]$ \\
\hline Data/restraints/parameters & $3996 / 1 / 271$ \\
\hline Goodness-of-fit on $\mathrm{F}^{2}$ & 1.053 \\
\hline Final $R$ indexes $[\mathrm{I}>=2 \sigma(\mathrm{I})]$ & $\mathrm{R}_{1}=0.0663, \mathrm{wR}_{2}=0.1605$ \\
\hline Final $\mathrm{R}$ indexes [all data] & $\mathrm{R}_{1}=0.0778, \mathrm{wR}_{2}=0.1729$ \\
\hline Largest diff. peak/hole/e $\AA^{-3}$ & $0.42 /-0.42$ \\
\hline
\end{tabular}



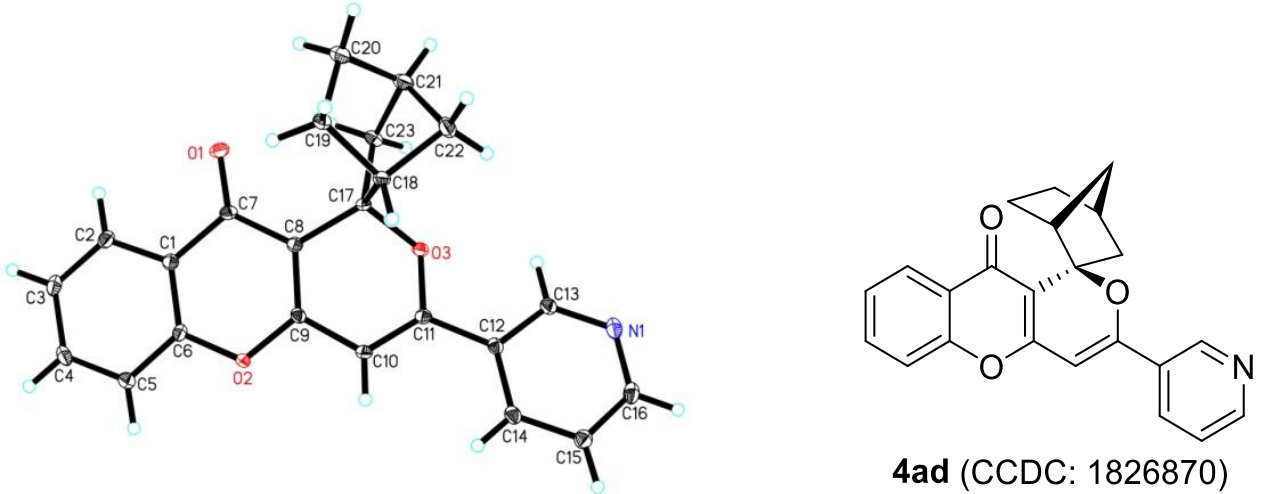

\begin{tabular}{|c|c|}
\hline Identification code & 4ad \\
\hline Empirical formula & $\mathrm{C}_{23} \mathrm{H}_{19} \mathrm{NO}_{3}$ \\
\hline Formula weight & 357.39 \\
\hline Temperature/K & $100.00(10)$ \\
\hline Crystal system & monoclinic \\
\hline Space group & $\mathrm{P} 2{ }_{1} / \mathrm{c}$ \\
\hline $\mathrm{a} / \AA ̊$ & $11.1173(7)$ \\
\hline $\mathrm{b} / \AA ̊$ & $11.3145(7)$ \\
\hline$c / \AA$ & $13.3338(8)$ \\
\hline$\alpha /{ }^{\circ}$ & 90 \\
\hline$\beta /{ }^{\circ}$ & $96.471(6)$ \\
\hline$\gamma /{ }^{\circ}$ & 90 \\
\hline Volume $/ \AA^{3}$ & $1666.53(18)$ \\
\hline $\mathrm{Z}$ & 4 \\
\hline$\rho_{\text {calc }} / \mathrm{cm}^{3}$ & 1.424 \\
\hline$\mu / \mathrm{mm}^{-1}$ & 0.094 \\
\hline $\mathrm{F}(000)$ & 752.0 \\
\hline Crystal size $/ \mathrm{mm}^{3}$ & $0.25 \times 0.2 \times 0.16$ \\
\hline Radiation & $\operatorname{MoK} \alpha(\lambda=0.71073)$ \\
\hline$\theta$ range for data collection ${ }^{\circ}$ & 3.403 to 29.518 \\
\hline Index ranges & $-15 \leq \mathrm{h} \leq 11,-15 \leq \mathrm{k} \leq 10,-18 \leq 1 \leq 16$ \\
\hline Reflections collected & 8739 \\
\hline Independent reflections & $3836\left[R_{\text {int }}=0.0540, R_{\text {sigma }}=0.0706\right]$ \\
\hline Data/restraints/parameters & $3836 / 0 / 244$ \\
\hline Goodness-of-fit on $\mathrm{F}^{2}$ & 1.037 \\
\hline Final $\mathrm{R}$ indexes $[\mathrm{I}>=2 \sigma(\mathrm{I})]$ & $\mathrm{R}_{1}=0.0622, \mathrm{wR}_{2}=0.1441$ \\
\hline Final R indexes [all data] & $\mathrm{R}_{1}=0.0857, \mathrm{wR}_{2}=0.1705$ \\
\hline Largest diff. peak/hole/e $\AA^{-3}$ & $0.65 /-0.36$ \\
\hline
\end{tabular}



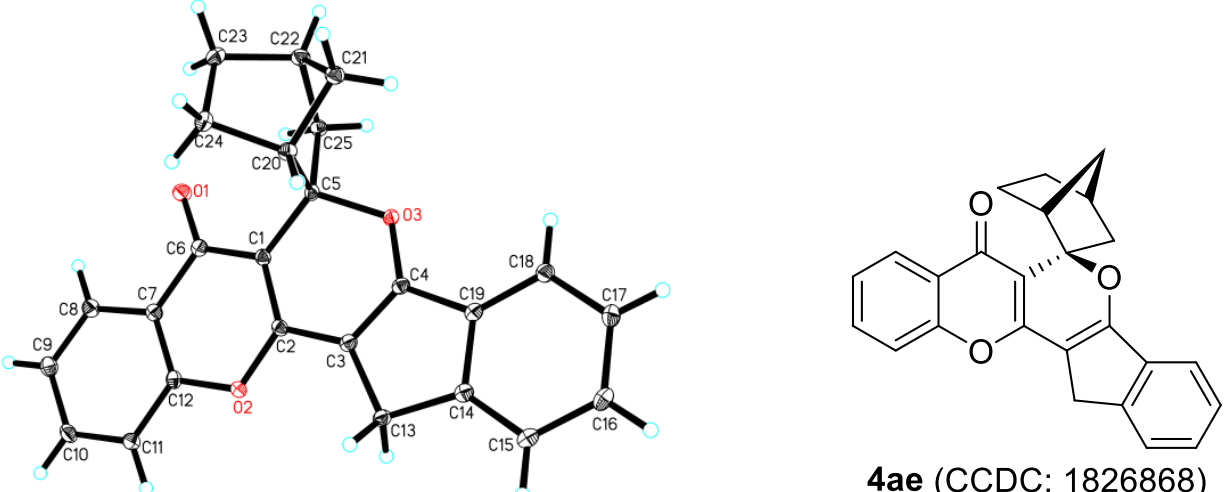

4ae (CCDC: 1826868)

\begin{tabular}{|c|c|}
\hline Identification code & 4ae \\
\hline Empirical formula & $\mathrm{C}_{25} \mathrm{H}_{20} \mathrm{O}_{3}$ \\
\hline Formula weight & 368.41 \\
\hline Temperature/K & $100.00(10)$ \\
\hline Crystal system & triclinic \\
\hline Space group & $\mathrm{P}-1$ \\
\hline $\mathrm{a} / \AA$ & $7.7518(4)$ \\
\hline $\mathrm{b} / \AA ̊$ & $9.4641(5)$ \\
\hline$c / \AA$ & $12.2238(7)$ \\
\hline$\alpha /{ }^{\circ}$ & $88.592(5)$ \\
\hline$\beta /{ }^{\circ}$ & $89.857(4)$ \\
\hline$\gamma /{ }^{\circ}$ & $77.459(5)$ \\
\hline Volume $/ \AA^{3}$ & $875.12(8)$ \\
\hline $\mathrm{Z}$ & 2 \\
\hline$\rho_{\text {calc }} \mathrm{g} / \mathrm{cm}^{3}$ & 1.398 \\
\hline$\mu / \mathrm{mm}^{-1}$ & 0.725 \\
\hline $\mathrm{F}(000)$ & 388.0 \\
\hline Crystal size $/ \mathrm{mm}^{3}$ & $0.2 \times 0.18 \times 0.14$ \\
\hline Radiation & $\mathrm{CuK} \alpha(\lambda=1.54184)$ \\
\hline$\theta$ range for data collection ${ }^{\circ}$ & 3.617 to 73.673 \\
\hline Index ranges & $-9 \leq \mathrm{h} \leq 6,-11 \leq \mathrm{k} \leq 9,-15 \leq 1 \leq 15$ \\
\hline Reflections collected & 5627 \\
\hline Independent reflections & $3411\left[R_{\text {int }}=0.0166, R_{\text {sigma }}=0.0237\right]$ \\
\hline Data/restraints/parameters & $3411 / 0 / 261$ \\
\hline Goodness-of-fit on $\mathrm{F}^{2}$ & 1.027 \\
\hline Final $R$ indexes $[\mathrm{I}>=2 \sigma(\mathrm{I})]$ & $\mathrm{R}_{1}=0.0390, \mathrm{wR}_{2}=0.1007$ \\
\hline Final $\mathrm{R}$ indexes [all data] & $\mathrm{R}_{1}=0.0421, \mathrm{wR}_{2}=0.1041$ \\
\hline Largest diff. peak/hole/e $\AA^{-3}$ & $0.30 /-0.26$ \\
\hline
\end{tabular}



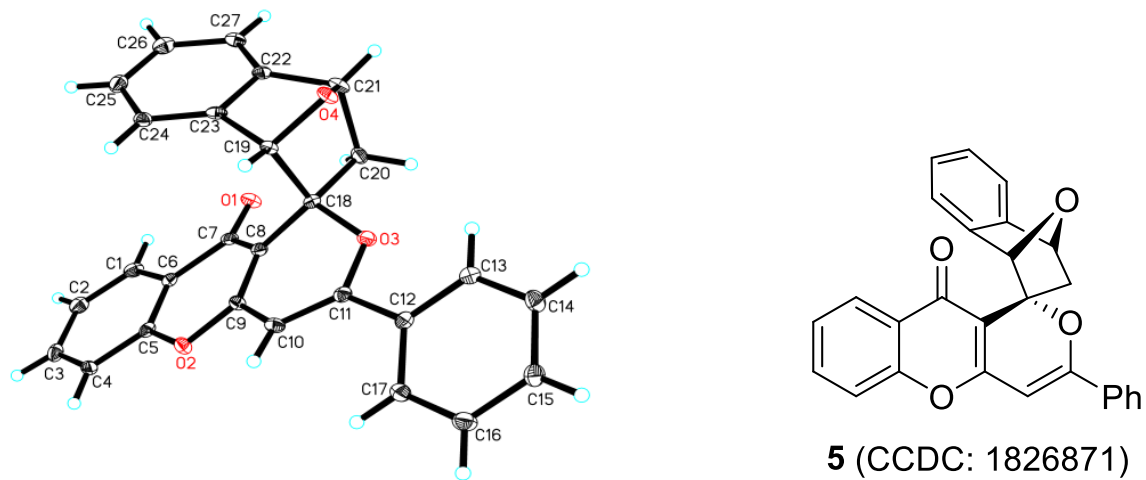

5 (CCDC: 1826871)

\begin{tabular}{|c|c|}
\hline Identification code & 5 \\
\hline Empirical formula & $\mathrm{C}_{27} \mathrm{H}_{18} \mathrm{O}_{4}$ \\
\hline Formula weight & 406.41 \\
\hline Temperature/K & $100.00(10)$ \\
\hline Crystal system & monoclinic \\
\hline Space group & $\mathrm{P} 2{ }_{1}$ \\
\hline $\mathrm{a} / \AA$ & $9.8585(5)$ \\
\hline $\mathrm{b} / \AA ̊ \AA$ & $17.8407(10)$ \\
\hline $\mathrm{c} / \AA$ & $11.1800(6)$ \\
\hline$\alpha /^{\circ}$ & 90 \\
\hline$\beta /{ }^{\circ}$ & $101.759(5)$ \\
\hline$\gamma /{ }^{\circ}$ & 90 \\
\hline Volume $/ \AA^{3}$ & $1925.10(18)$ \\
\hline $\mathrm{Z}$ & 4 \\
\hline$\rho_{\text {calc }} \mathrm{g} / \mathrm{cm}^{3}$ & 1.402 \\
\hline$\mu / \mathrm{mm}^{-1}$ & 0.094 \\
\hline $\mathrm{F}(000)$ & 848.0 \\
\hline Crystal size $/ \mathrm{mm}^{3}$ & $0.14 \times 0.12 \times 0.11$ \\
\hline Radiation & $\operatorname{MoK} \alpha(\lambda=0.71073)$ \\
\hline $2 \theta$ range for data collection $/^{\circ}$ & 4.22 to 58.876 \\
\hline Index ranges & $-12 \leq \mathrm{h} \leq 9,-16 \leq \mathrm{k} \leq 24,-15 \leq 1 \leq 14$ \\
\hline Reflections collected & 10304 \\
\hline Independent reflections & $6442\left[\mathrm{R}_{\text {int }}=0.0360, \mathrm{R}_{\text {sigma }}=0.0622\right]$ \\
\hline Data/restraints/parameters & $6442 / 1 / 559$ \\
\hline Goodness-of-fit on $\mathrm{F}^{2}$ & 1.032 \\
\hline Final $R$ indexes $[I>=2 \sigma(I)]$ & $\mathrm{R}_{1}=0.0534, \mathrm{wR}_{2}=0.1214$ \\
\hline Final R indexes [all data] & $\mathrm{R}_{1}=0.0697, \mathrm{wR}_{2}=0.1340$ \\
\hline Largest diff. peak/hole/e $\AA^{-3}$ & $0.27 /-0.30$ \\
\hline Flack parameter & $0.3(10)$ \\
\hline
\end{tabular}



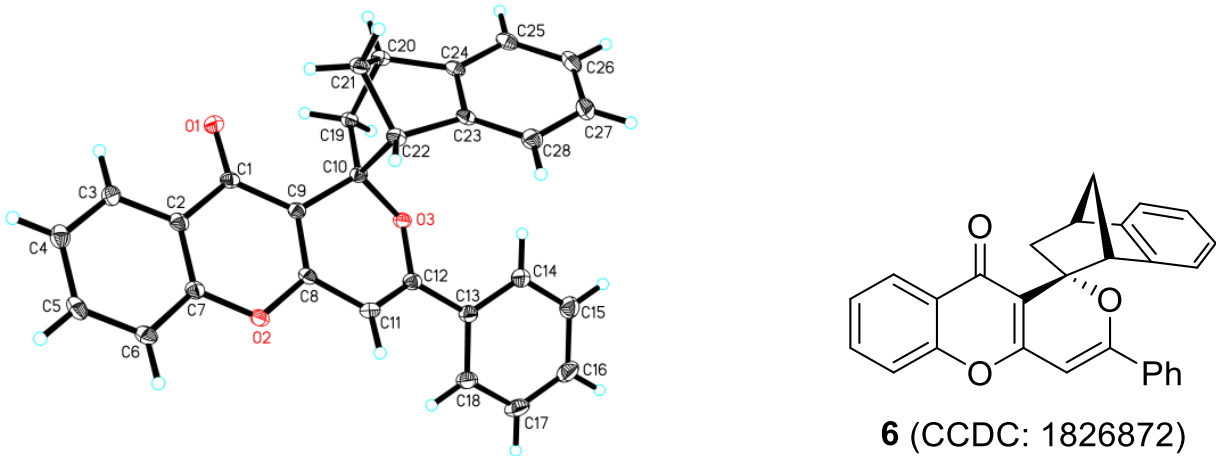

6 (CCDC: 1826872)

\begin{tabular}{|c|c|}
\hline Identification code & 6 \\
\hline Empirical formula & $\mathrm{C}_{28} \mathrm{H}_{20} \mathrm{O}_{3}$ \\
\hline Formula weight & 404.44 \\
\hline Temperature/K & $100.01(10)$ \\
\hline Crystal system & monoclinic \\
\hline Space group & Pn \\
\hline $\mathrm{a} / \AA ̊$ & $10.4846(14)$ \\
\hline $\mathrm{b} / \AA$ & $5.4604(10)$ \\
\hline$c / \AA$ & $17.182(2)$ \\
\hline$\alpha /{ }^{\circ}$ & 90 \\
\hline$\beta /{ }^{\circ}$ & $93.357(13)$ \\
\hline$\gamma /{ }^{\circ}$ & 90 \\
\hline Volume $/ \AA^{3}$ & $982.0(3)$ \\
\hline $\mathrm{Z}$ & 2 \\
\hline$\rho_{\text {calc }} \mathrm{g} / \mathrm{cm}^{3}$ & 1.368 \\
\hline$\mu / \mathrm{mm}^{-1}$ & 0.088 \\
\hline $\mathrm{F}(000)$ & 424.0 \\
\hline Crystal size $/ \mathrm{mm}^{3}$ & $0.13 \times 0.12 \times 0.11$ \\
\hline Radiation & $\operatorname{MoK} \alpha(\lambda=0.71073)$ \\
\hline $2 \theta$ range for data collection $/^{\circ}$ & 4.438 to 59.214 \\
\hline Index ranges & $-14 \leq \mathrm{h} \leq 9,-7 \leq \mathrm{k} \leq 6,-23 \leq 1 \leq 20$ \\
\hline Reflections collected & 4653 \\
\hline Independent reflections & $2947\left[R_{\text {int }}=0.0313, R_{\text {sigma }}=0.0505\right]$ \\
\hline Data/restraints/parameters & $2947 / 2 / 280$ \\
\hline Goodness-of-fit on $\mathrm{F}^{2}$ & 1.020 \\
\hline Final $R$ indexes $[\mathrm{I}>=2 \sigma(\mathrm{I})]$ & $\mathrm{R}_{1}=0.0423, \mathrm{wR}_{2}=0.0928$ \\
\hline Final $\mathrm{R}$ indexes [all data] & $\mathrm{R}_{1}=0.0490, \mathrm{wR}_{2}=0.0999$ \\
\hline Largest diff. peak/hole / e $\AA^{-3}$ & $0.19 /-0.25$ \\
\hline
\end{tabular}



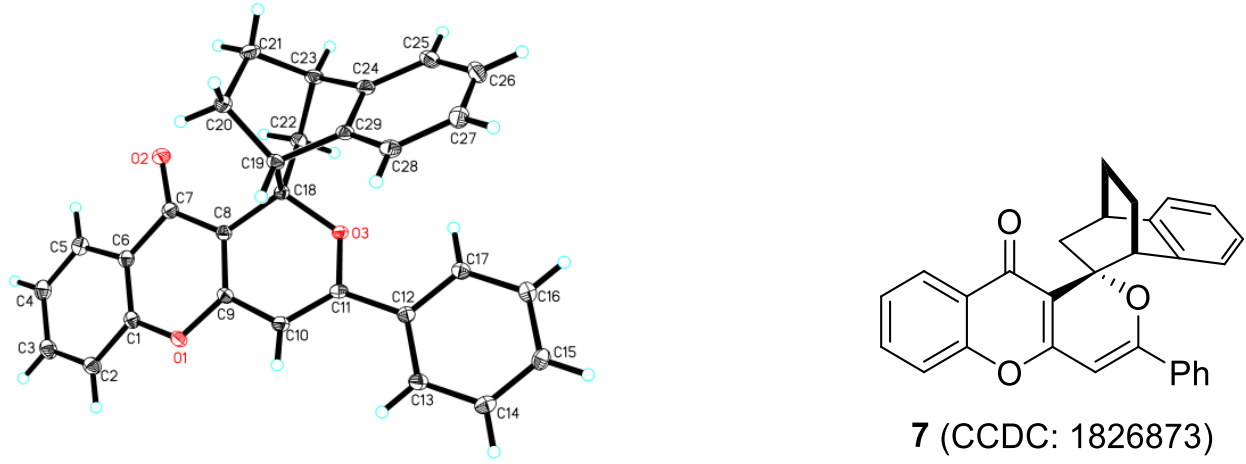

\begin{tabular}{|c|c|}
\hline Identification code & 7 \\
\hline Empirical formula & $\mathrm{C}_{29} \mathrm{H}_{22} \mathrm{O}_{3}$ \\
\hline Formula weight & 418.46 \\
\hline Temperature/K & $100.00(10)$ \\
\hline Crystal system & monoclinic \\
\hline Space group & $\mathrm{P} 2{ }_{1} / \mathrm{c}$ \\
\hline $\mathrm{a} / \AA ̊$ & $9.8879(2)$ \\
\hline $\mathrm{b} / \AA$ & $18.3761(3)$ \\
\hline$c / \AA$ & $11.4277(2)$ \\
\hline$\alpha /{ }^{\circ}$ & 90 \\
\hline$\beta /{ }^{\circ}$ & $100.069(2)$ \\
\hline$\gamma /{ }^{\circ}$ & 90 \\
\hline Volume $/ \AA^{3}$ & 2044.44(7) \\
\hline $\mathrm{Z}$ & 4 \\
\hline$\rho_{\text {calc }} \mathrm{g} / \mathrm{cm}^{3}$ & 1.360 \\
\hline$\mu / \mathrm{mm}^{-1}$ & 0.691 \\
\hline $\mathrm{F}(000)$ & 880.0 \\
\hline Crystal size $/ \mathrm{mm}^{3}$ & $0.13 \times 0.12 \times 0.11$ \\
\hline Radiation & $\mathrm{CuK} \alpha(\lambda=1.54184)$ \\
\hline $2 \theta$ range for data collection $/^{\circ}$ & 9.084 to 147.732 \\
\hline Index ranges & $-12 \leq \mathrm{h} \leq 11,-13 \leq \mathrm{k} \leq 22,-14 \leq 1 \leq 13$ \\
\hline Reflections collected & 7367 \\
\hline Independent reflections & $3985\left[R_{\text {int }}=0.0217, R_{\text {sigma }}=0.0256\right]$ \\
\hline Data/restraints/parameters & $3985 / 0 / 289$ \\
\hline Goodness-of-fit on $\mathrm{F}^{2}$ & 1.064 \\
\hline Final $\mathrm{R}$ indexes $[\mathrm{I}>=2 \sigma(\mathrm{I})]$ & $\mathrm{R}_{1}=0.0494, \mathrm{wR}_{2}=0.1295$ \\
\hline Final R indexes [all data] & $\mathrm{R}_{1}=0.0516, \mathrm{wR}_{2}=0.1314$ \\
\hline Largest diff. peak/hole/e $\AA^{-3}$ & $0.31 /-0.33$ \\
\hline
\end{tabular}



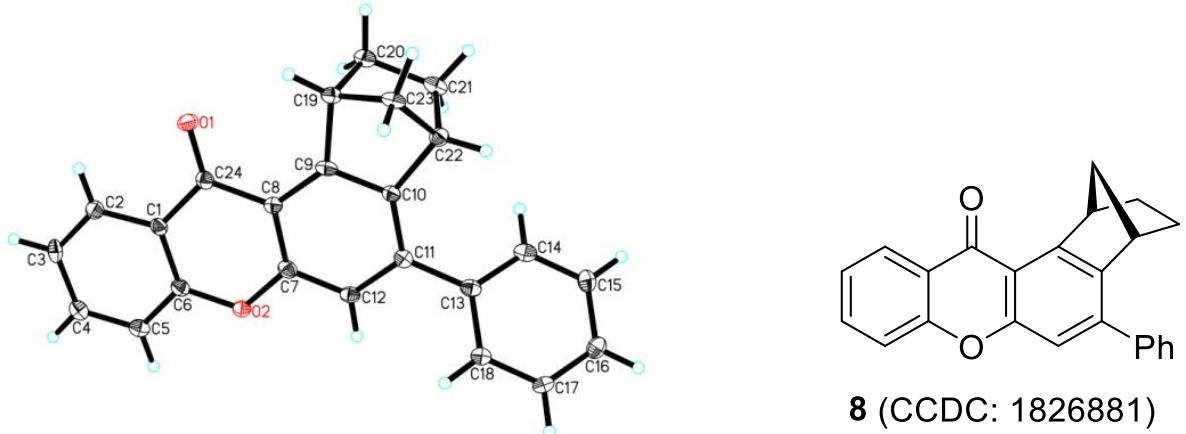

\begin{tabular}{|c|c|}
\hline Identification code & 8 \\
\hline Empirical formula & $\mathrm{C}_{24} \mathrm{H}_{18} \mathrm{O}_{2}$ \\
\hline Formula weight & 338.38 \\
\hline Temperature/K & $100.00(10)$ \\
\hline Crystal system & monoclinic \\
\hline Space group & $\mathrm{P} 2{ }_{1} / \mathrm{c}$ \\
\hline $\mathrm{a} / \AA$ & $26.879(6)$ \\
\hline $\mathrm{b} / \AA ̊ \AA$ & $7.4058(16)$ \\
\hline $\mathrm{c} / \AA$ & $16.892(3)$ \\
\hline$\alpha /^{\circ}$ & 90 \\
\hline$\beta /{ }^{\circ}$ & $90.065(19)$ \\
\hline$\gamma /{ }^{\circ}$ & 90 \\
\hline Volume $/ \AA^{3}$ & $3362.6(12)$ \\
\hline $\mathrm{Z}$ & 8 \\
\hline$\rho_{\text {calc }} \mathrm{g} / \mathrm{cm}^{3}$ & 1.337 \\
\hline$\mu / \mathrm{mm}^{-1}$ & 0.084 \\
\hline $\mathrm{F}(000)$ & 1424.0 \\
\hline Crystal size $/ \mathrm{mm}^{3}$ & $0.13 \times 0.12 \times 0.11$ \\
\hline Radiation & $\operatorname{MoK} \alpha(\lambda=0.71073)$ \\
\hline $2 \theta$ range for data collection $/^{\circ}$ & 4.546 to 59.19 \\
\hline Index ranges & $-31 \leq \mathrm{h} \leq 36,-10 \leq \mathrm{k} \leq 10,-19 \leq 1 \leq 23$ \\
\hline Reflections collected & 17401 \\
\hline Independent reflections & $7776\left[R_{\text {int }}=0.1796, R_{\text {sigma }}=0.1977\right]$ \\
\hline Data/restraints/parameters & $7776 / 0 / 469$ \\
\hline Goodness-of-fit on $\mathrm{F}^{2}$ & 1.072 \\
\hline Final $R$ indexes $[I>=2 \sigma(I)]$ & $\mathrm{R}_{1}=0.1110, \mathrm{wR}_{2}=0.2618$ \\
\hline Final R indexes [all data] & $\mathrm{R}_{1}=0.2070, \mathrm{wR}_{2}=0.3143$ \\
\hline Largest diff. peak/hole/e $\AA^{-3}$ & $0.38 /-0.36$ \\
\hline
\end{tabular}



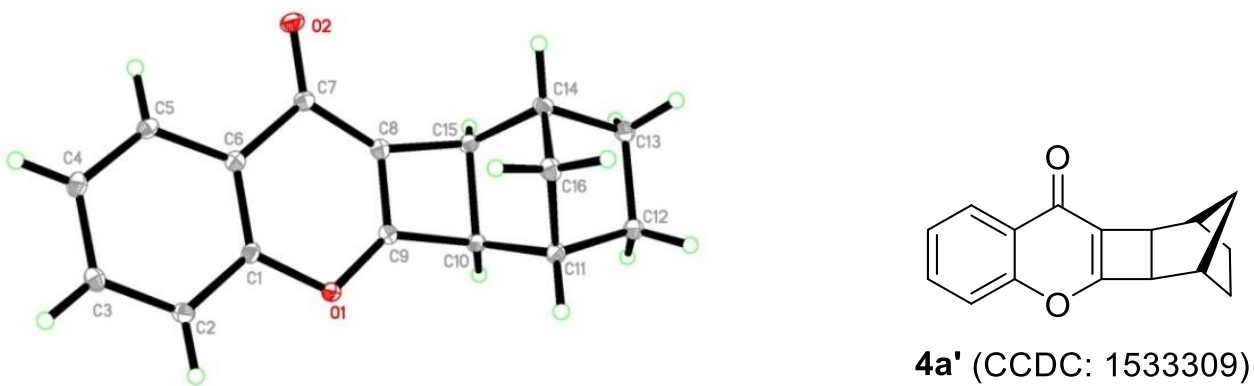

\begin{tabular}{|c|c|}
\hline Identification code & $4 \mathbf{a}^{\prime}$ \\
\hline Empirical formula & $\mathrm{C}_{16} \mathrm{H}_{14} \mathrm{O}_{2}$ \\
\hline Formula weight & 238.27 \\
\hline Temperature/K & $100.01(10)$ \\
\hline Crystal system & monoclinic \\
\hline Space group & $\mathrm{P} 2{ }_{1} / \mathrm{c}$ \\
\hline $\mathrm{a} / \AA$ & $9.3133(2)$ \\
\hline $\mathrm{b} / \AA ̊ \AA$ & $11.0394(2)$ \\
\hline $\mathrm{c} / \AA ̊$ & $11.6092(2)$ \\
\hline$\alpha /{ }^{\circ}$ & 90 \\
\hline$\beta /{ }^{\circ}$ & $106.331(2)$ \\
\hline$\gamma /{ }^{\circ}$ & 90 \\
\hline Volume $/ \AA^{3}$ & $1145.42(4)$ \\
\hline $\mathrm{Z}$ & 4 \\
\hline$\rho_{\text {calc }} \mathrm{g} / \mathrm{cm}^{3}$ & 1.382 \\
\hline$\mu / \mathrm{mm}^{-1}$ & 0.718 \\
\hline $\mathrm{F}(000)$ & 504.0 \\
\hline Crystal size $/ \mathrm{mm}^{3}$ & $0.3 \times 0.2 \times 0.2$ \\
\hline Radiation & $\mathrm{CuK} \alpha(\lambda=1.54184)$ \\
\hline$\theta$ range for data collection $/^{\circ}$ & 9.896 to 146.802 \\
\hline Index ranges & $-11 \leq \mathrm{h} \leq 9,-9 \leq \mathrm{k} \leq 13,-12 \leq 1 \leq 14$ \\
\hline Reflections collected & 4149 \\
\hline Independent reflections & $2245\left[\mathrm{R}_{\mathrm{int}}=0.0145, \mathrm{R}_{\text {sigma }}=0.0177\right]$ \\
\hline Data/restraints/parameters & $2245 / 0 / 163$ \\
\hline Goodness-of-fit on $\mathrm{F}^{2}$ & 1.032 \\
\hline Final $R$ indexes $[I>=2 \sigma(I)]$ & $\mathrm{R}_{1}=0.0395, \mathrm{wR}_{2}=0.1034$ \\
\hline Final $\mathrm{R}$ indexes [all data] & $\mathrm{R}_{1}=0.0411, \mathrm{wR}_{2}=0.1049$ \\
\hline Largest diff. peak/hole/e $\AA^{-3}$ & $0.25 /-0.25$ \\
\hline
\end{tabular}


11. ${ }^{1} \mathrm{H}$ and ${ }^{13} \mathrm{C}$ NMR spectra of $4 \mathrm{a}-\mathrm{ae}, 5-9$, and $4 \mathrm{a}^{\prime}$. ysy-135-H

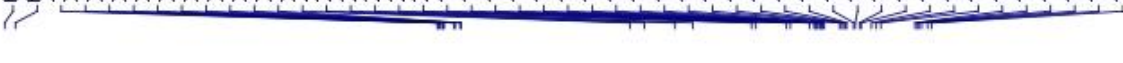

Scheme 2, 4a
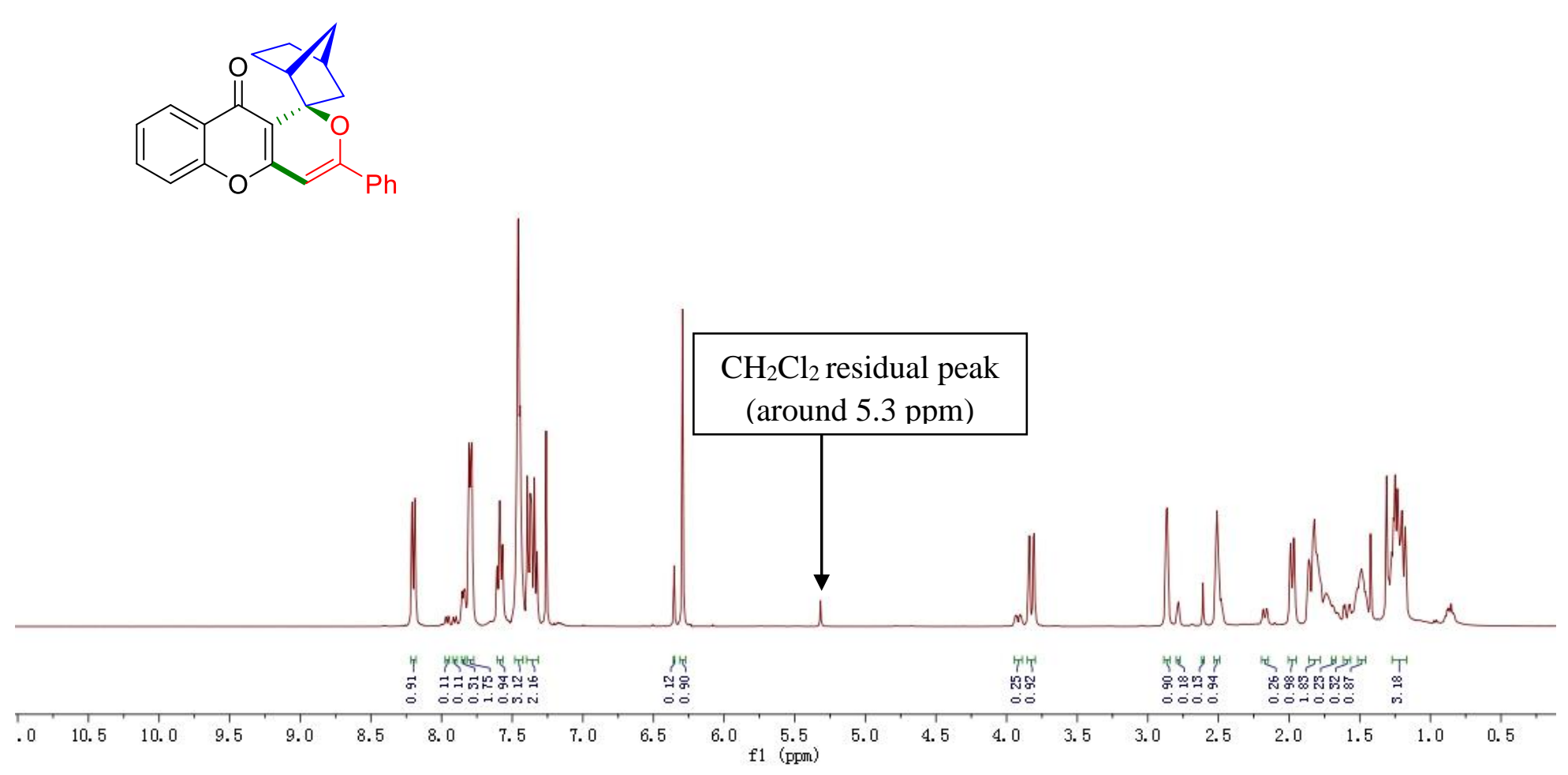
Scheme 2, 4a
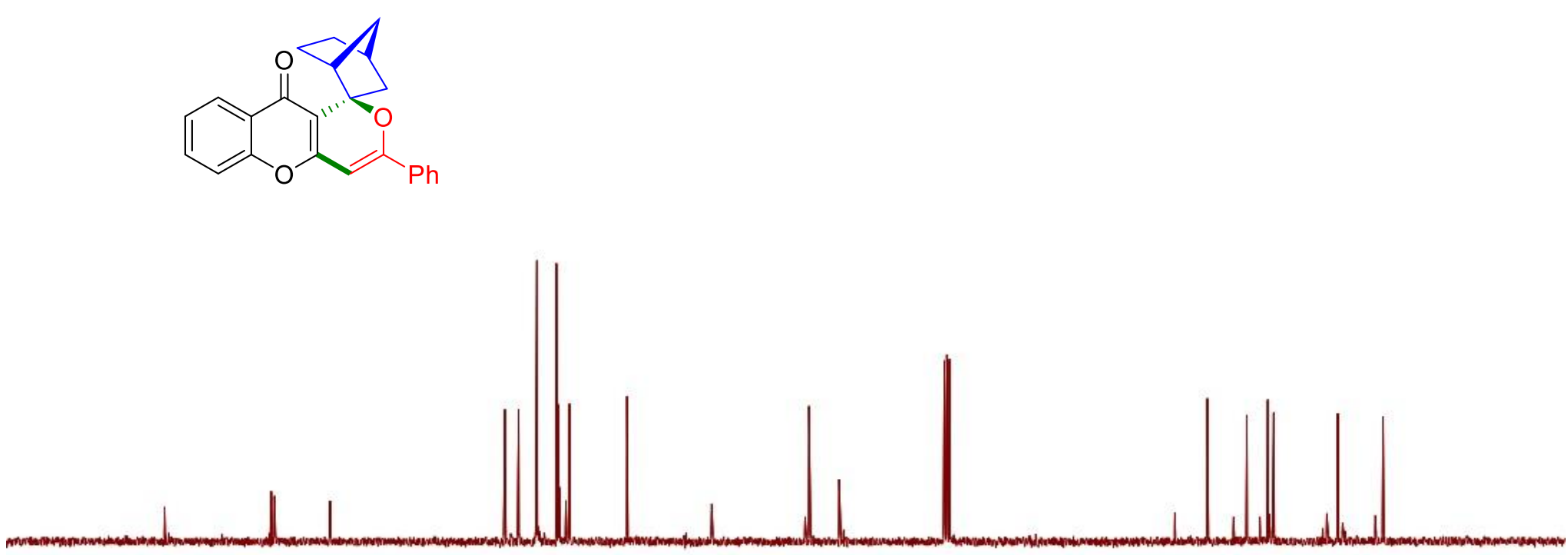

190 
Scheme 2, 4b
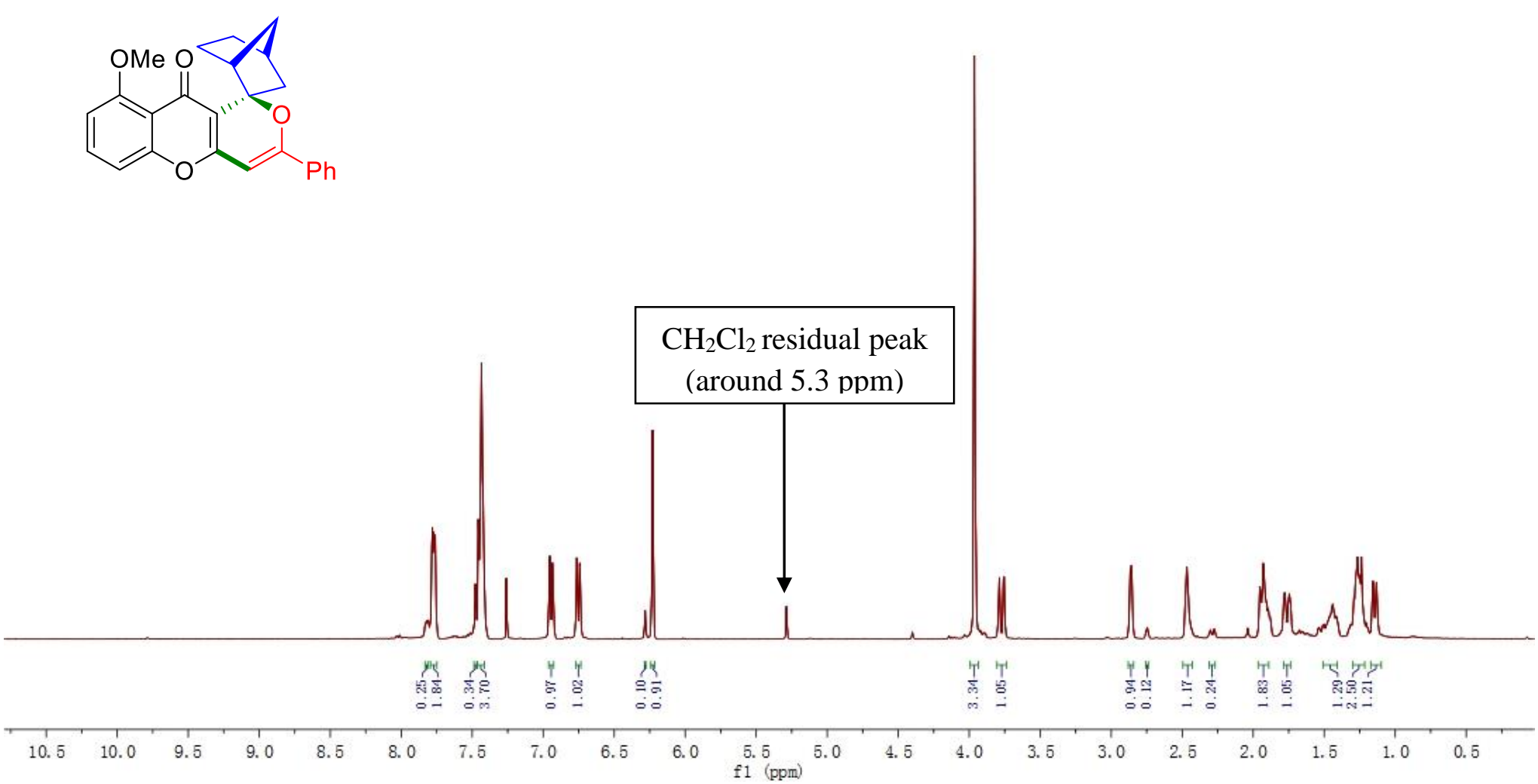


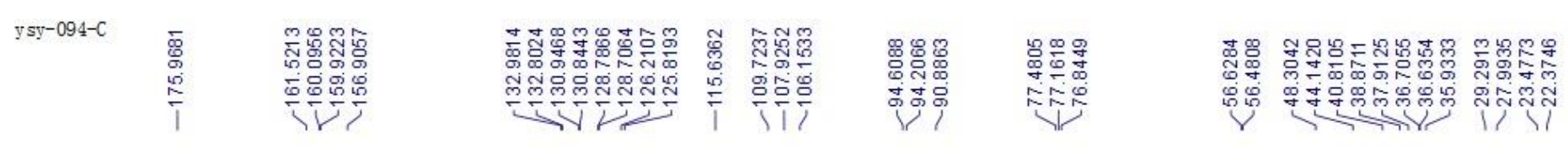

Scheme 2, 4b
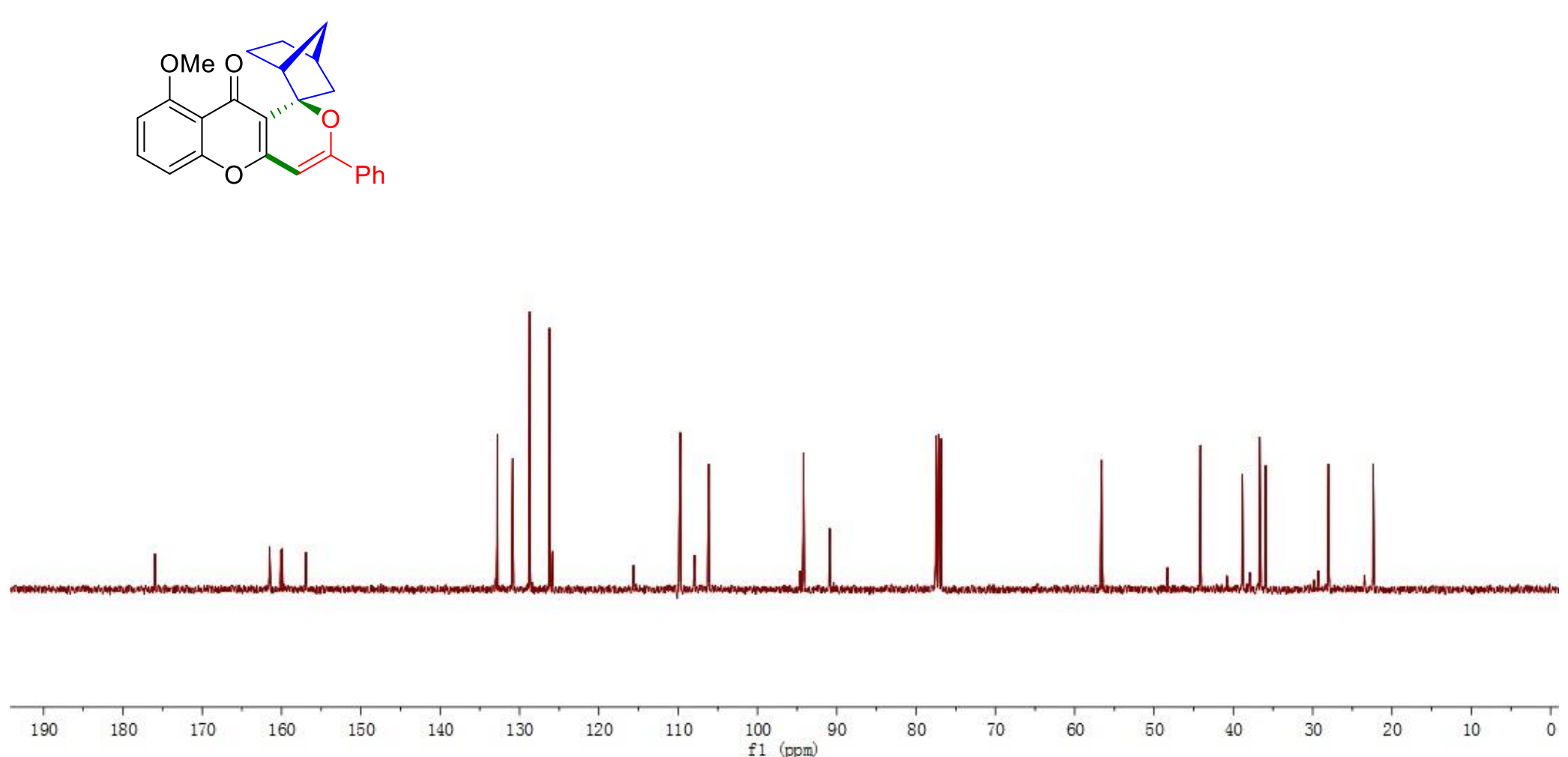
Scheme 2, 4c
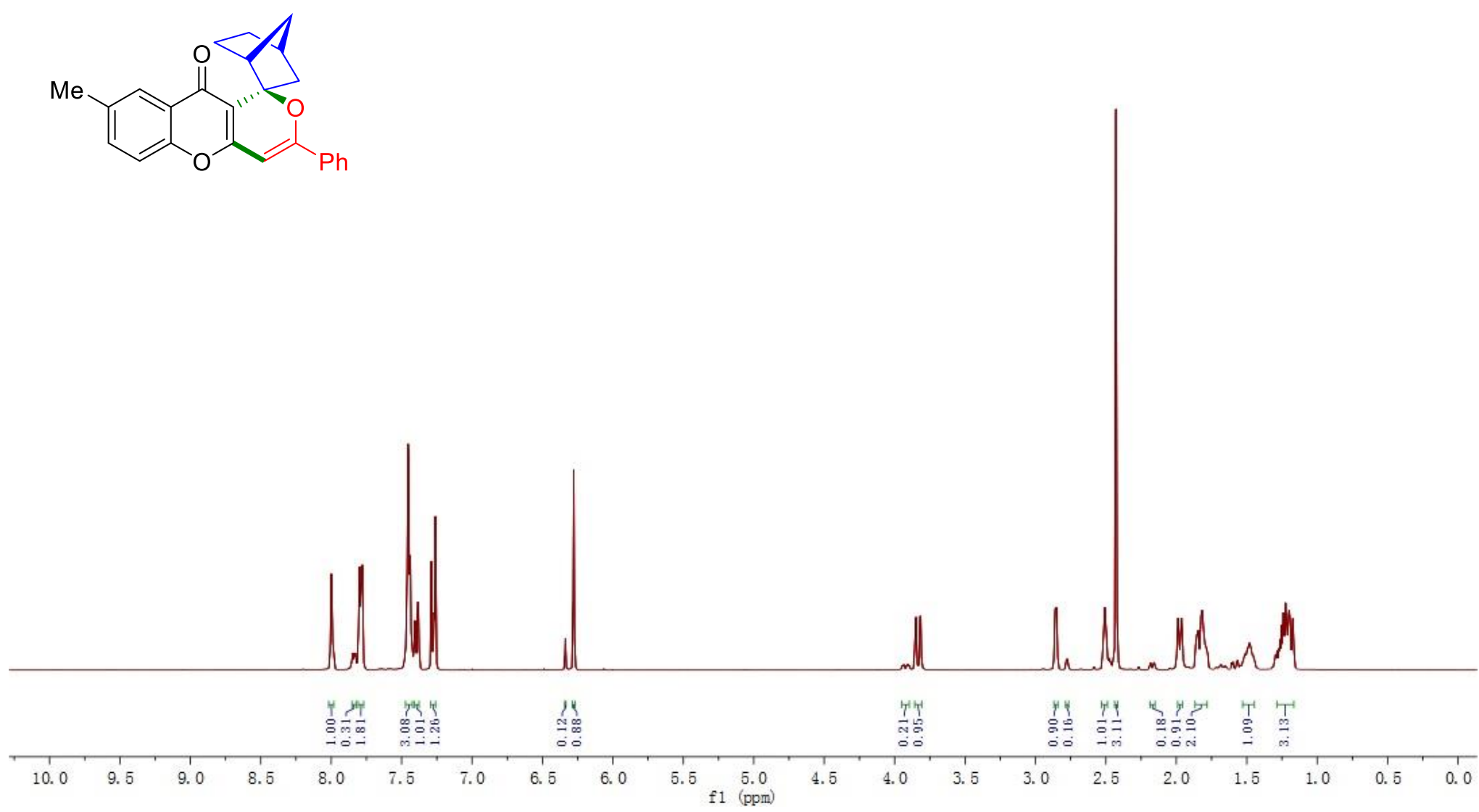
Scheme 2, 4c

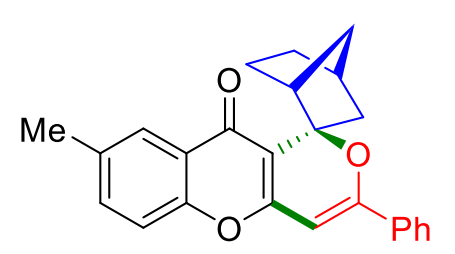

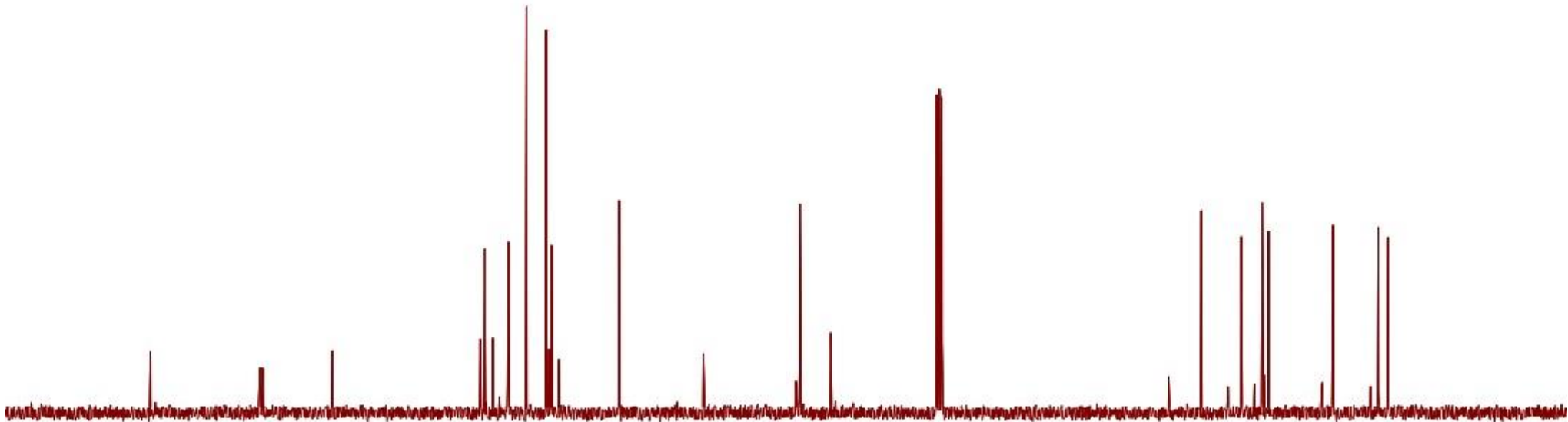


Scheme 2, 4d
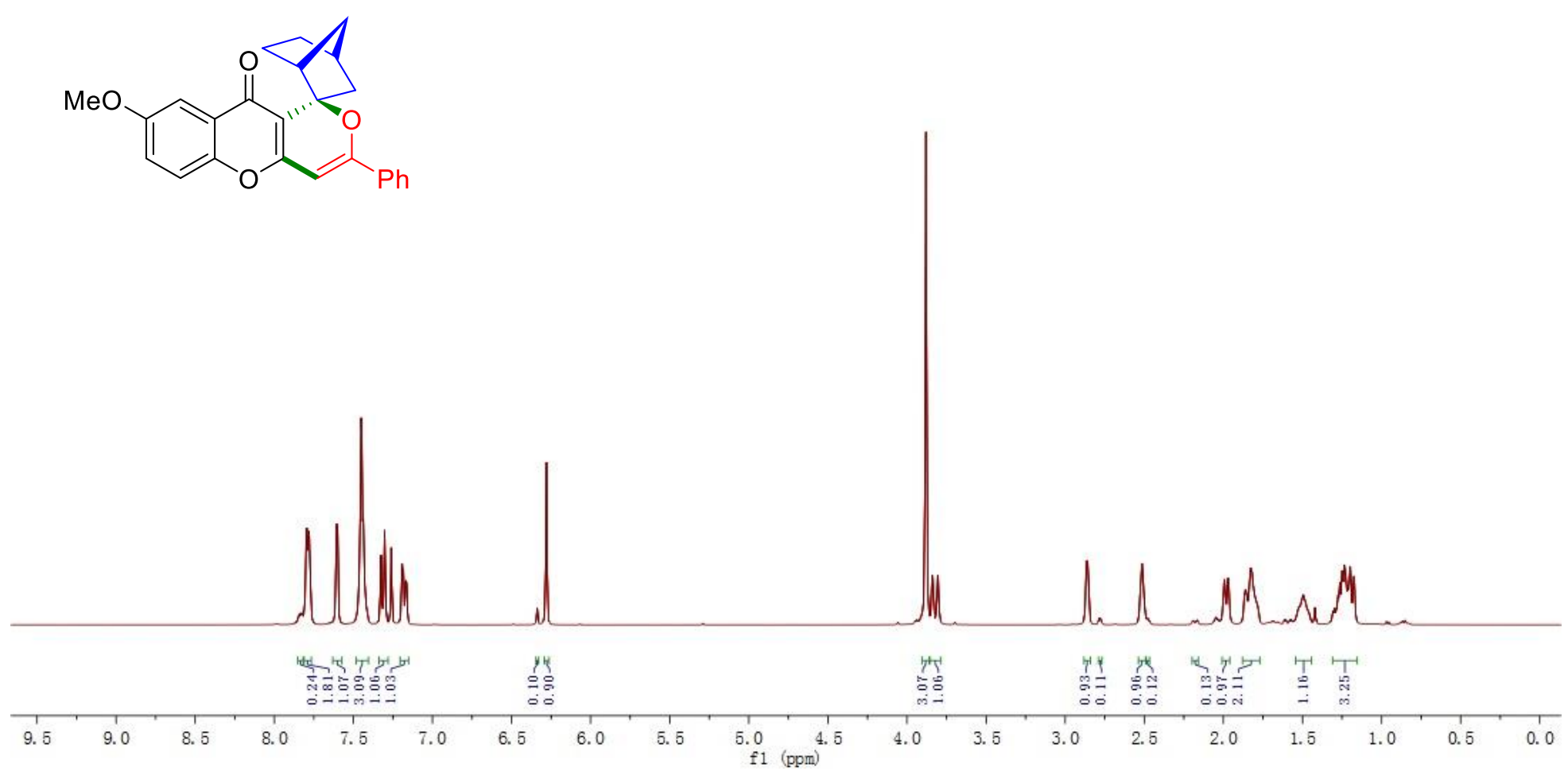
Scheme 2, 4d<smiles>COC1=CC=C2OC3=Cc4oc5ccc(cc5c(=O)c4OC(c4ccccc4)=C3)CC2C1</smiles>

|||||||| 


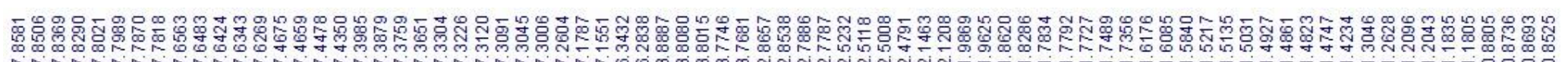

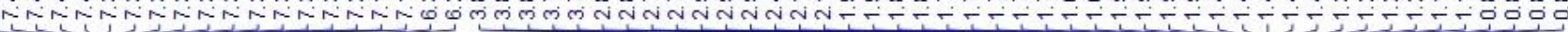

Scheme 2, 4e
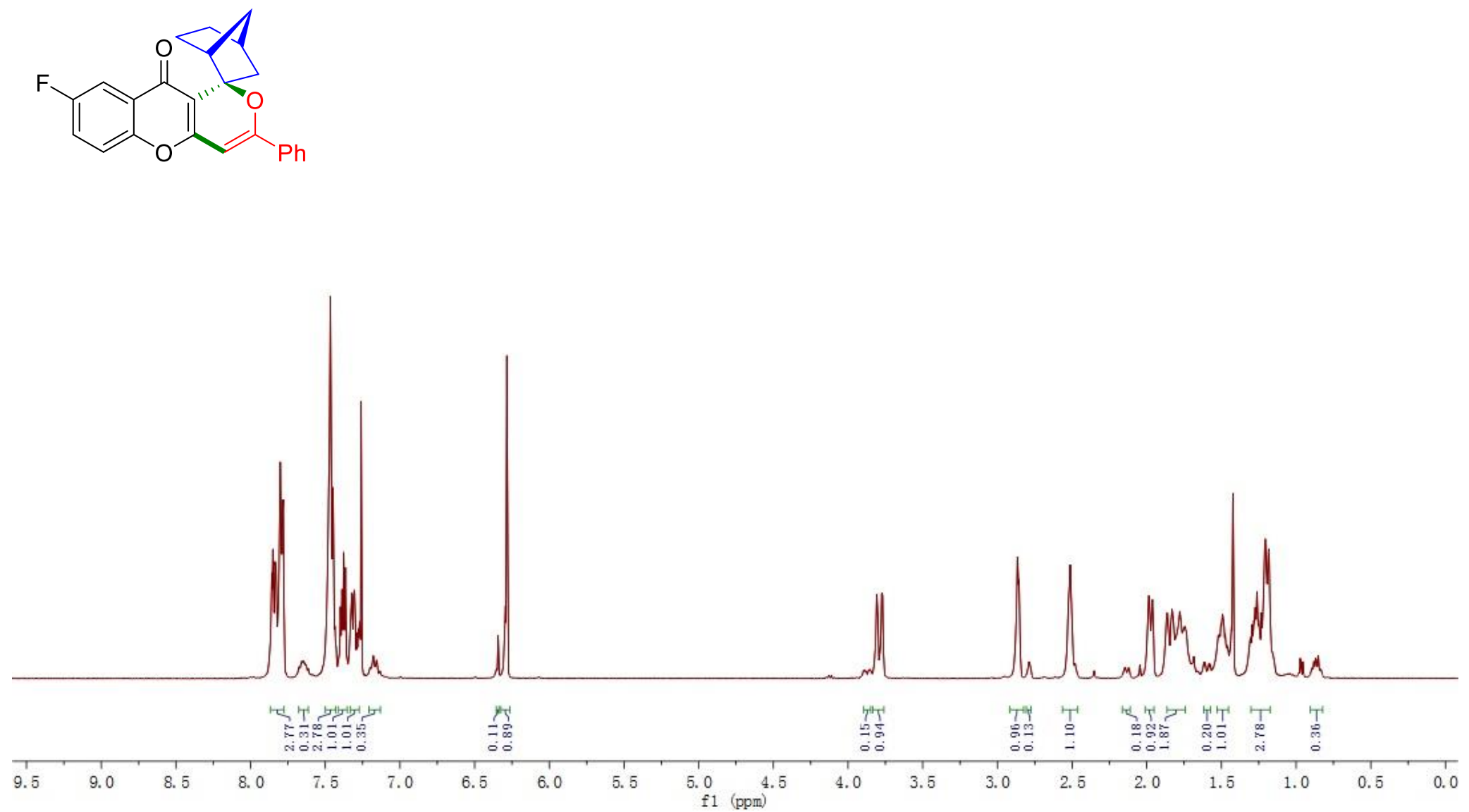


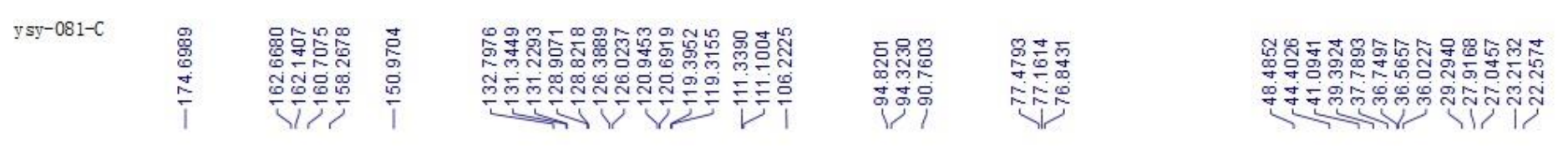

Scheme 2, 4e
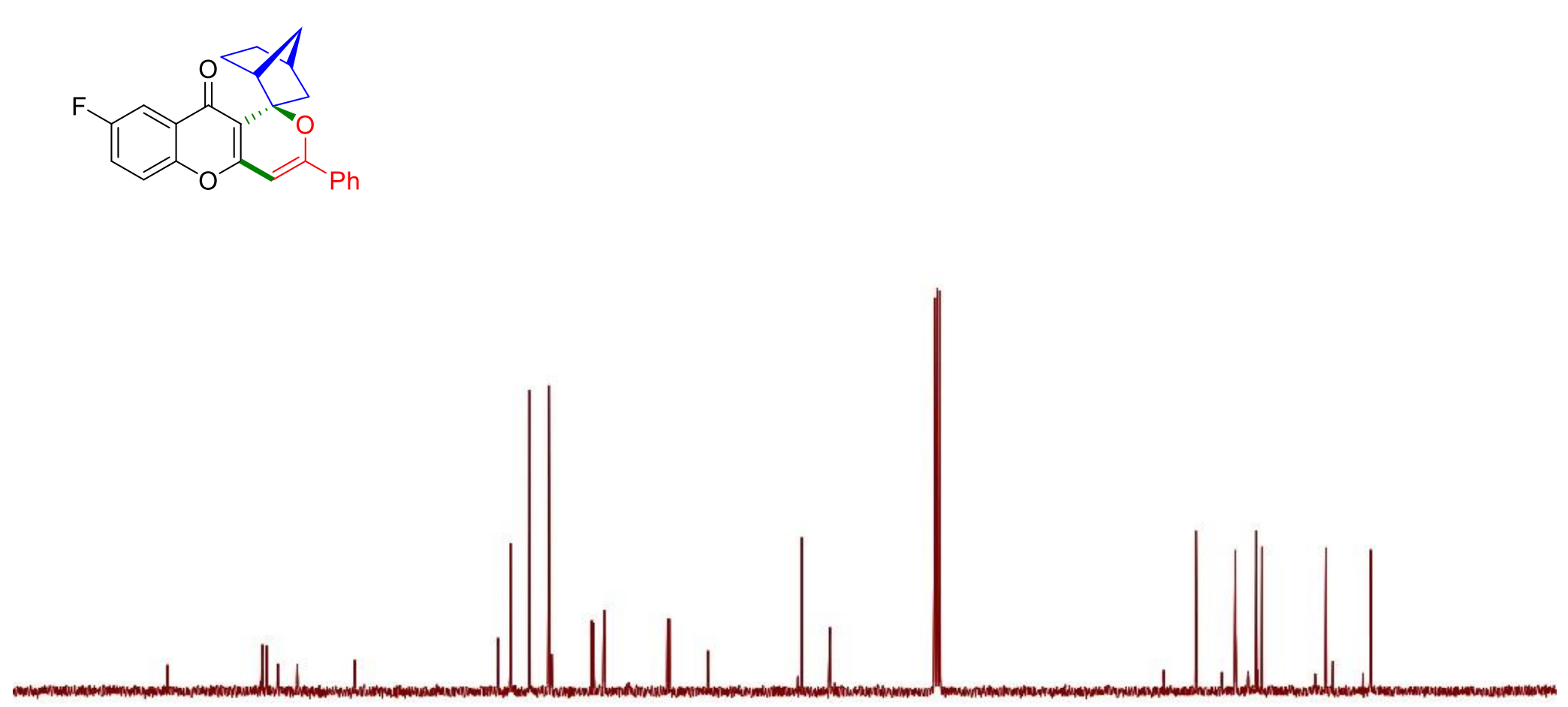

$190 \quad 180 \quad 170$

$160 \quad 150$

$140 \quad 130$

$120 \quad 110$

$100 \quad 90$

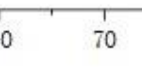

60

50

30

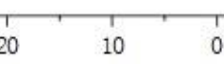


Scheme 2, $4 \mathrm{f}$
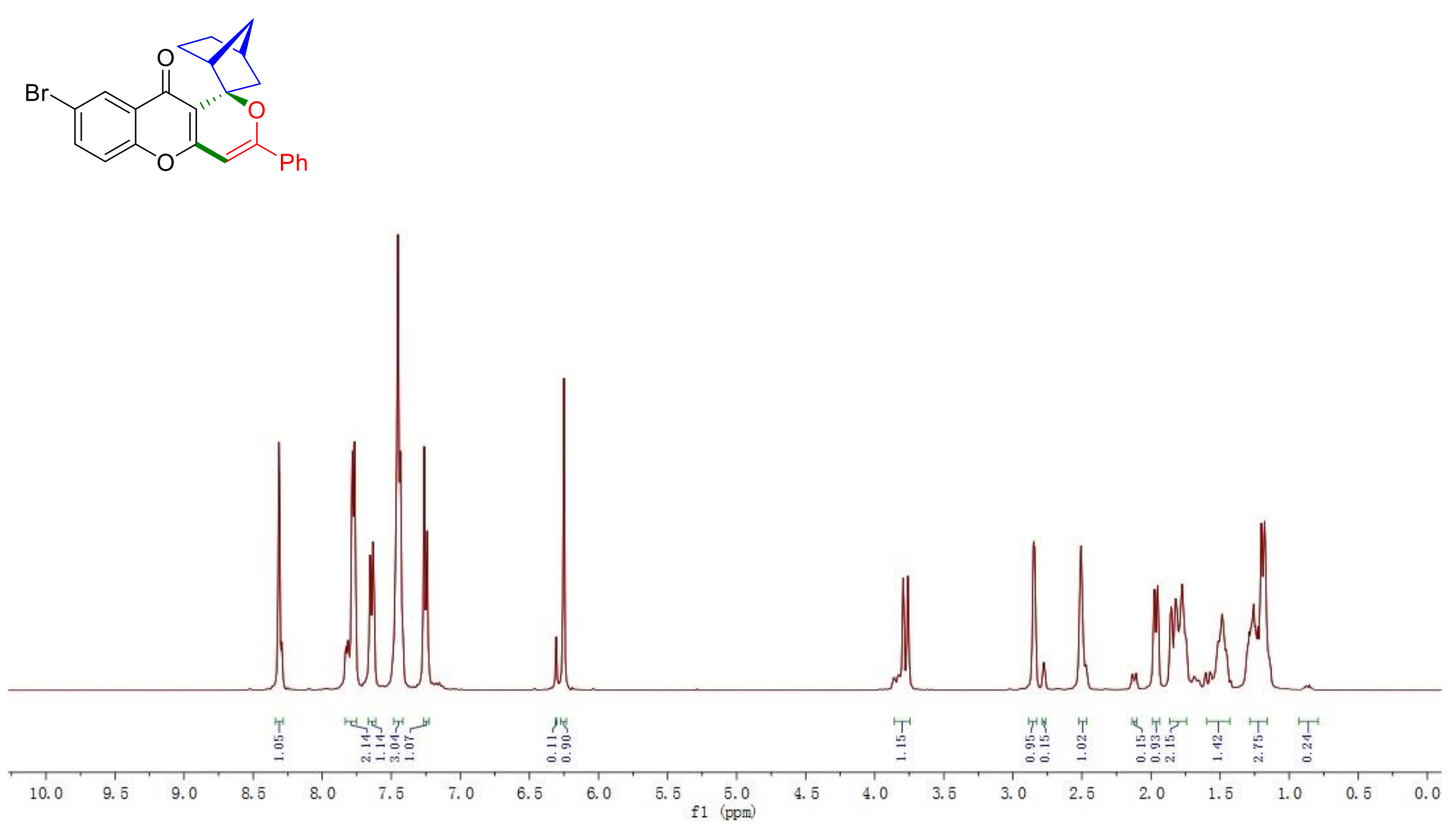


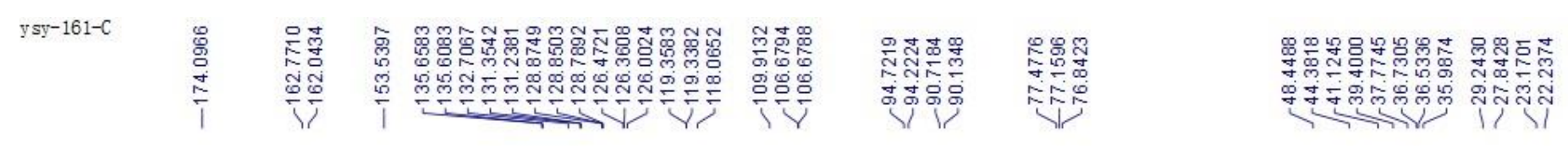

Scheme 2, $4 f$
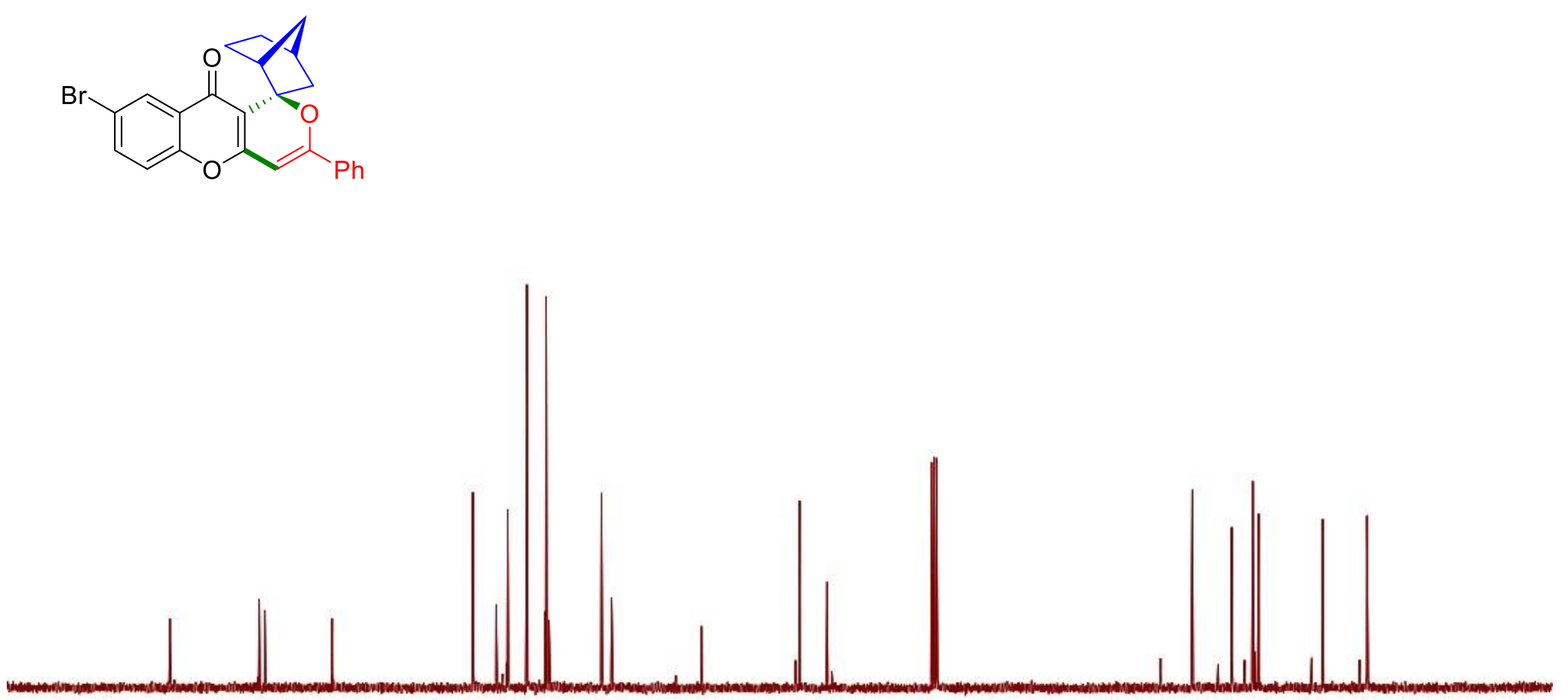


\section{Scheme $2,4 \mathrm{~g}$}
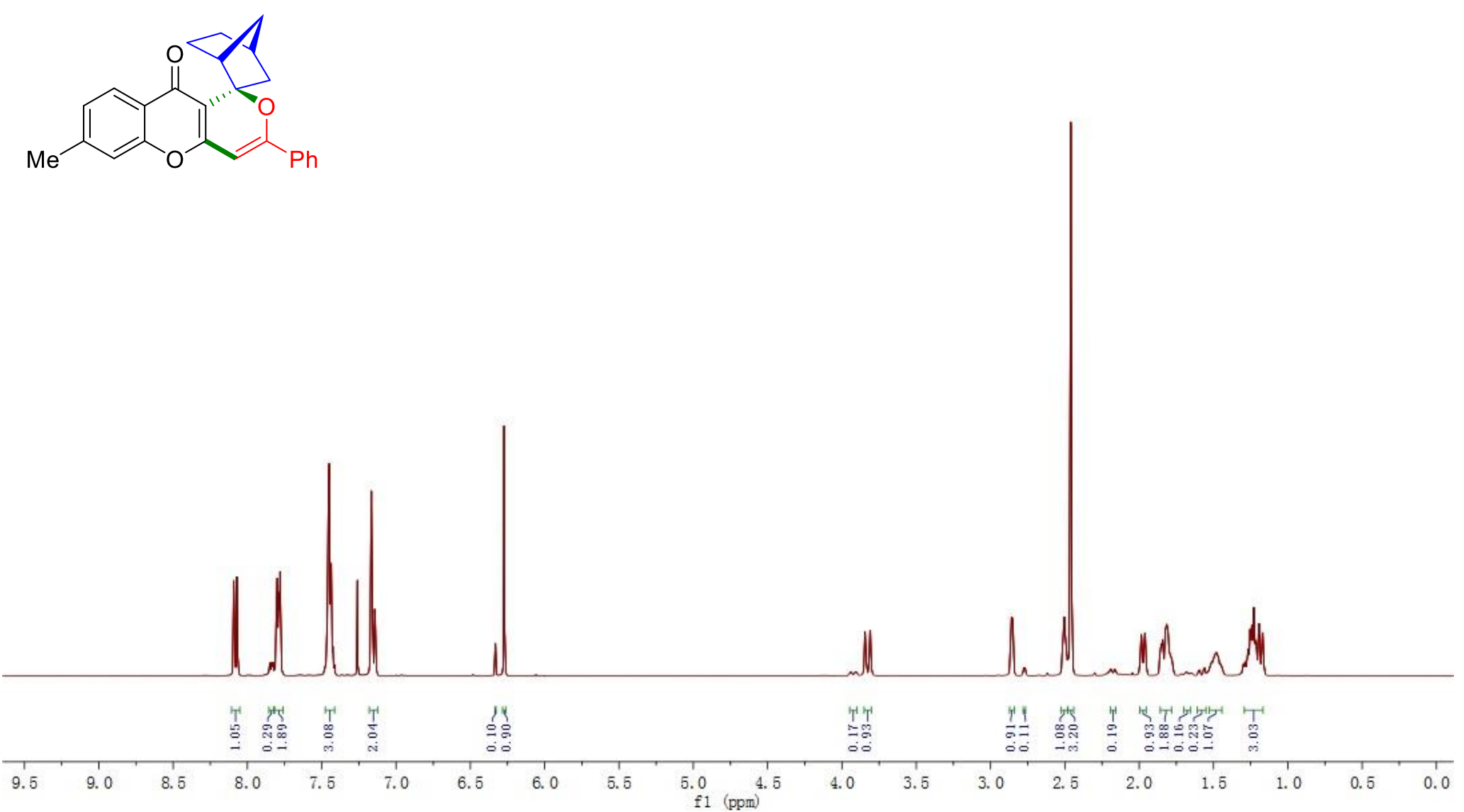
Scheme 2, 4g

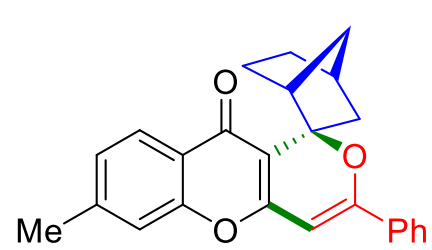

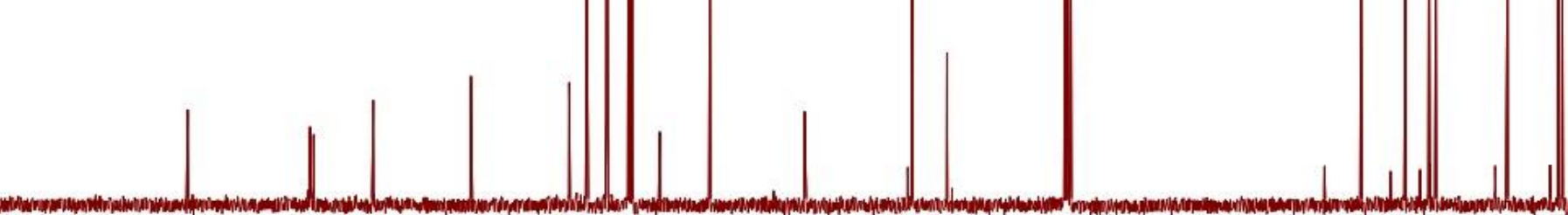

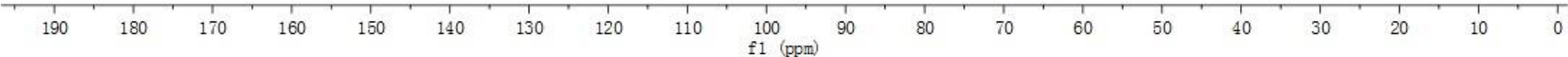


y $s y-078-H$

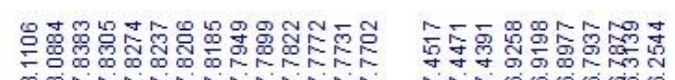

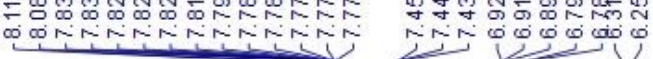

Scheme 2, 4h
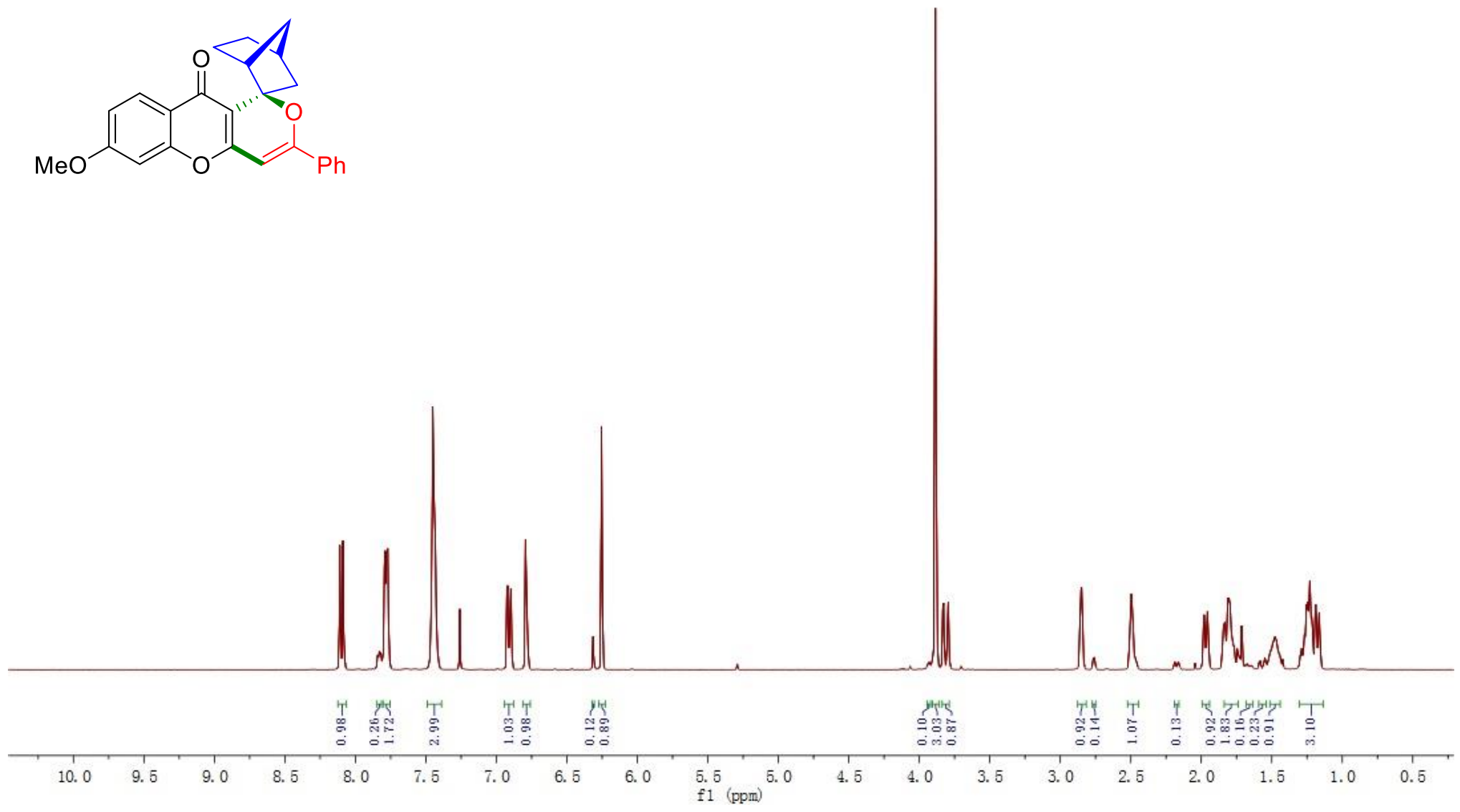

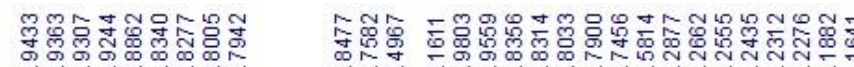

.

पु

NNN N-T-T- 


\section{Scheme 2, 4h}

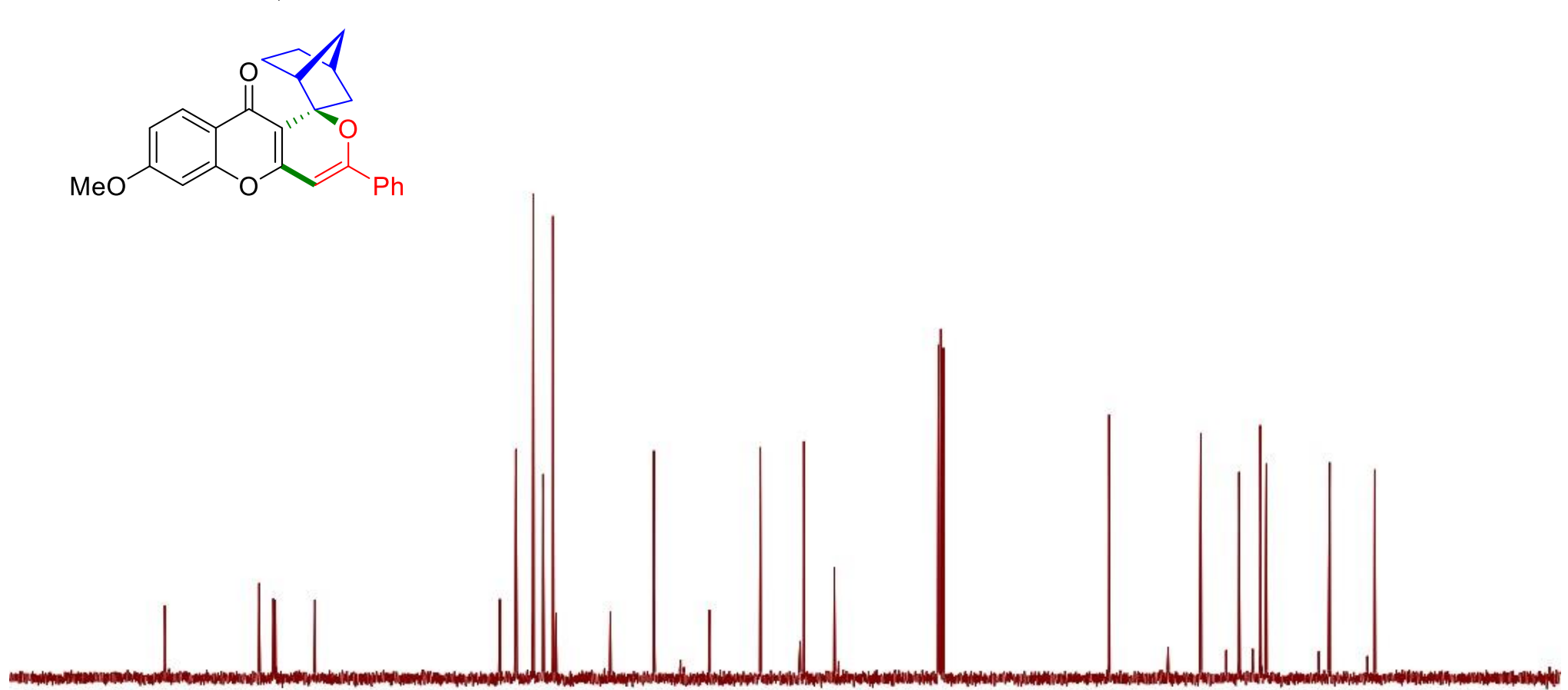




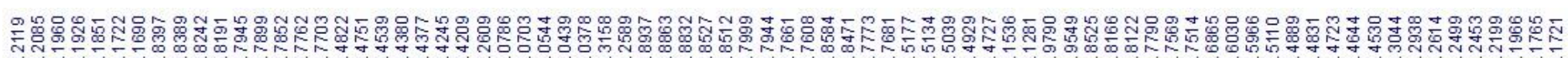

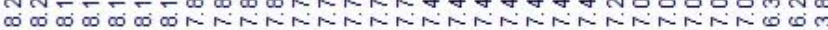

Scheme 2, 4i
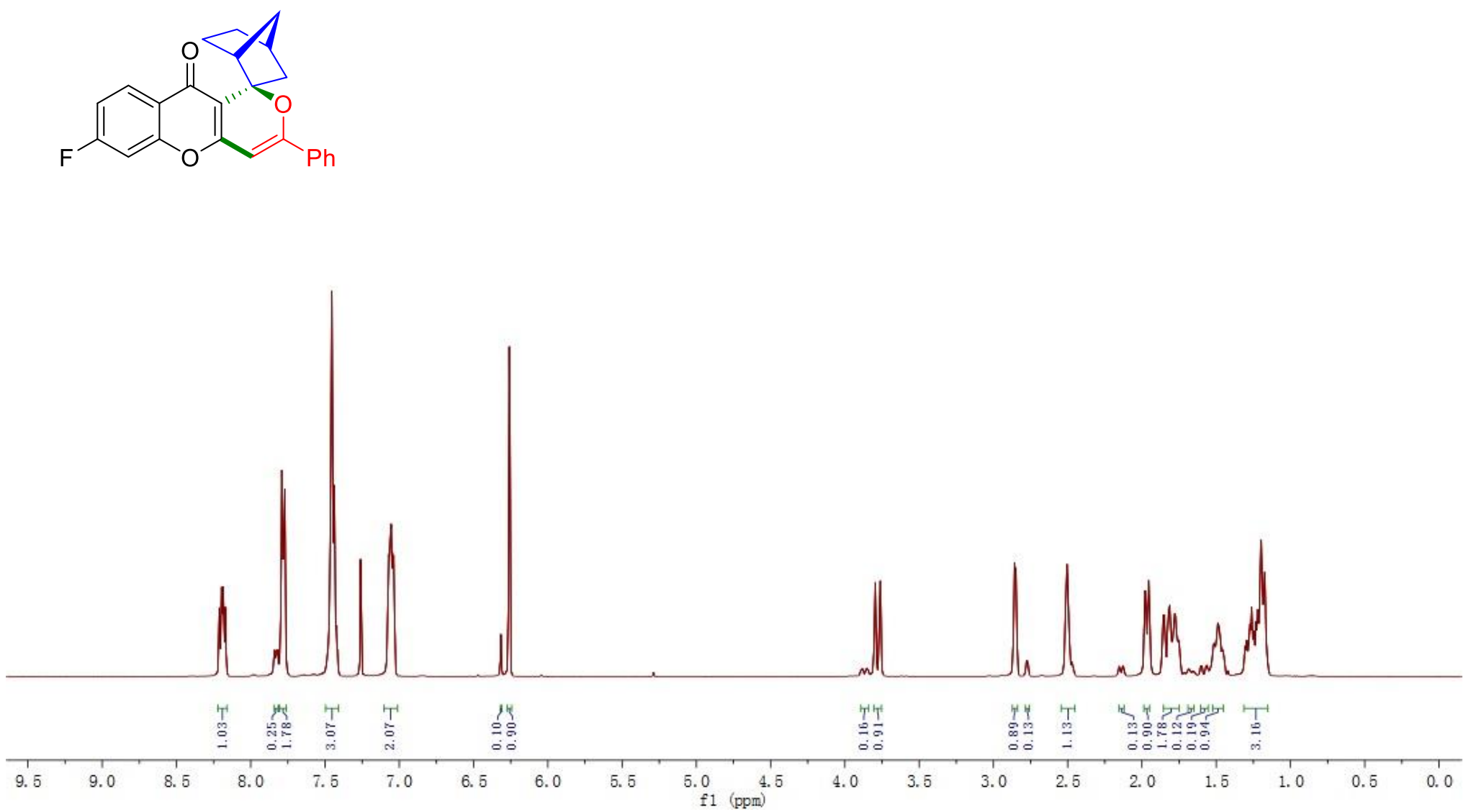
Scheme 2, 4i
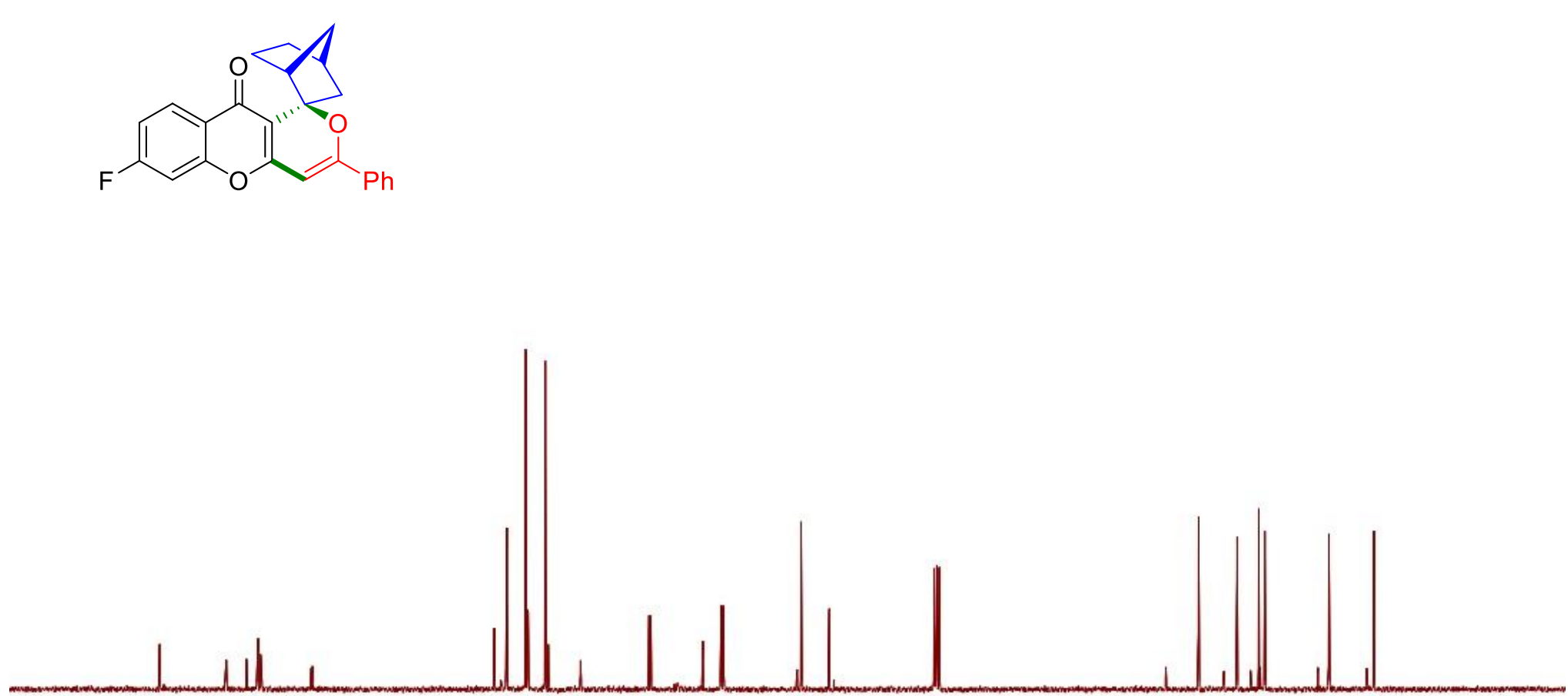

190

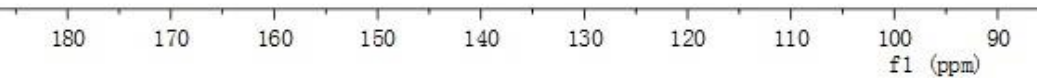


Scheme 2, 4j

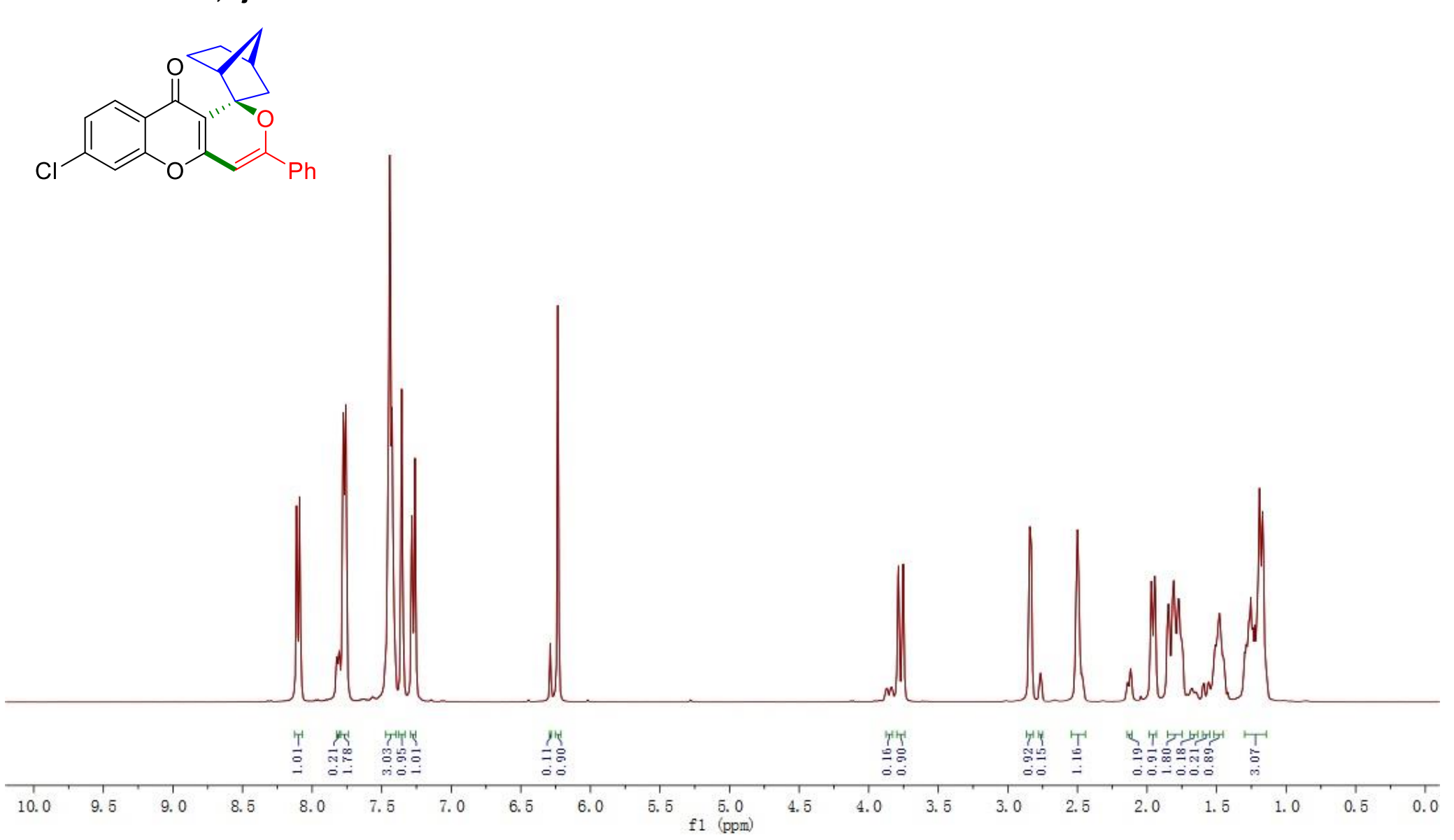




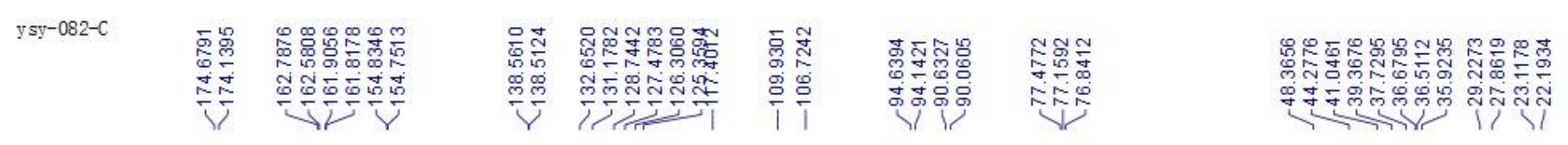

Scheme 2, 4j
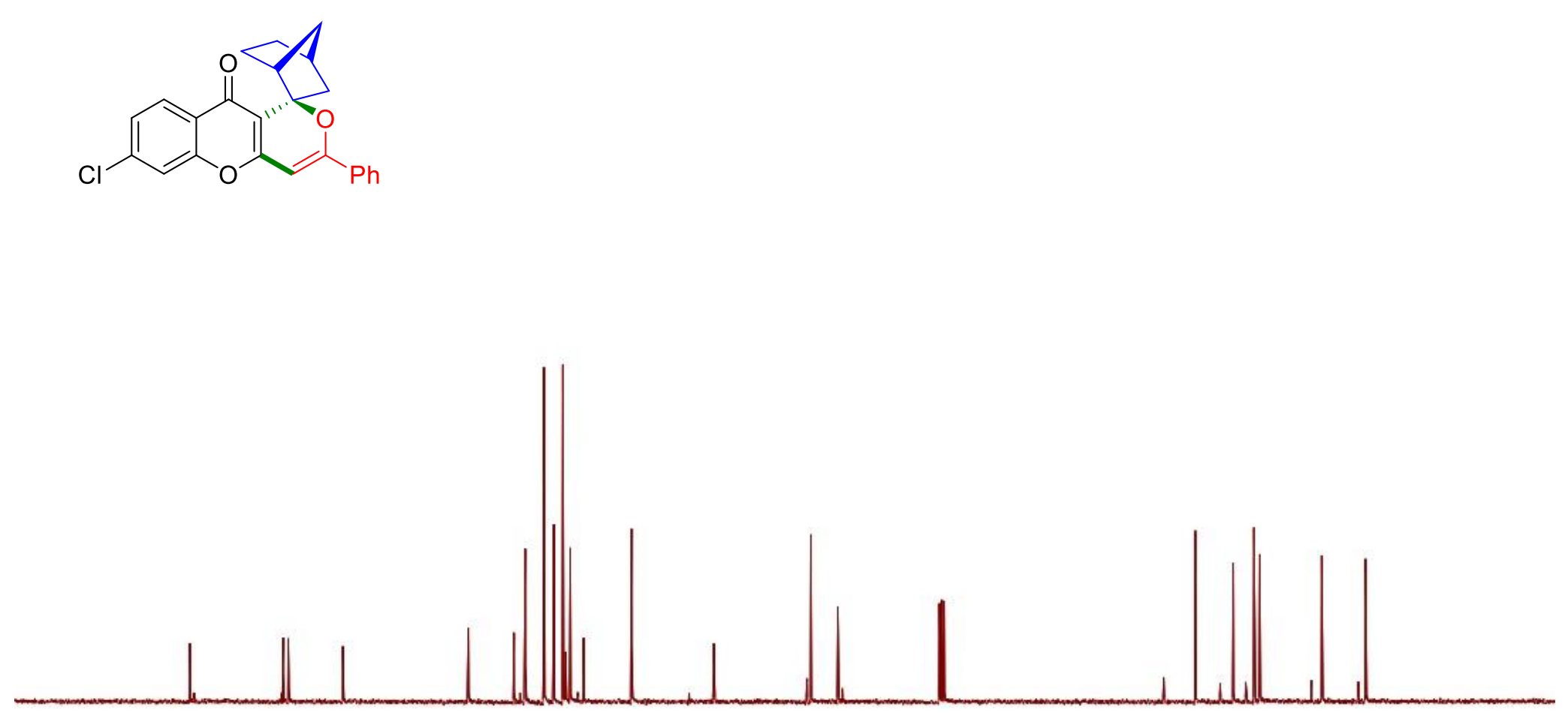
Scheme 2, 4k
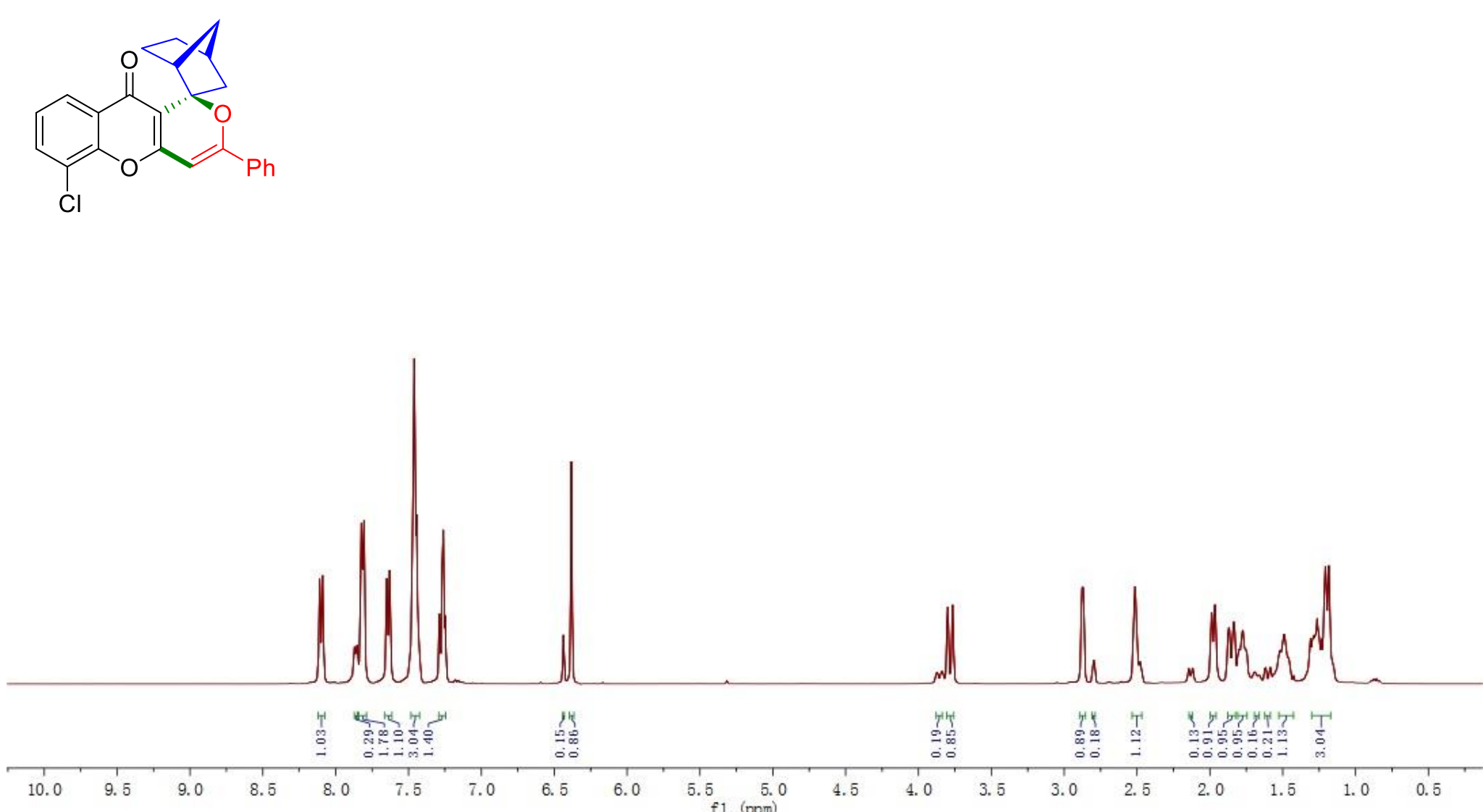
ysy-160-c

Scheme 2, 4k
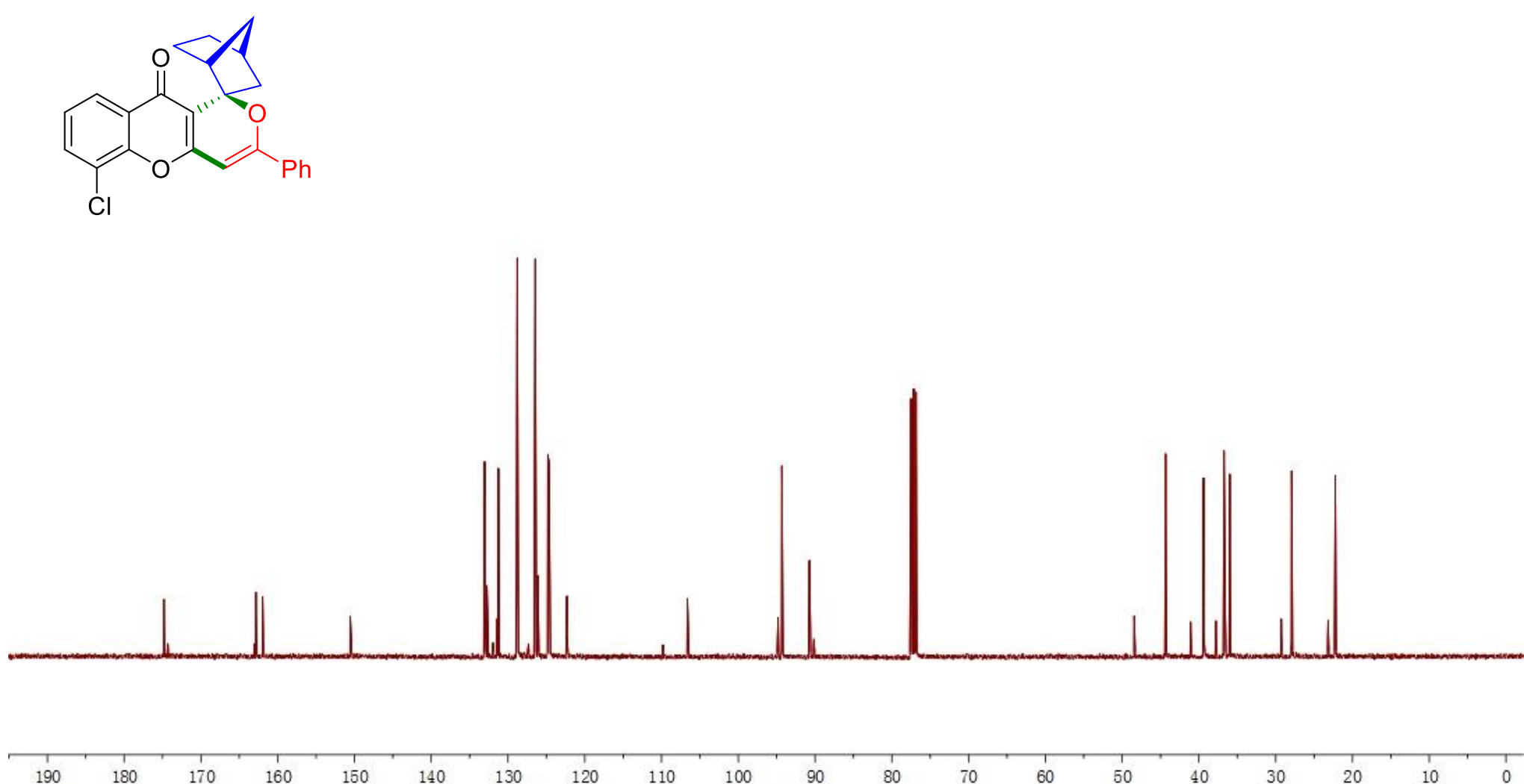


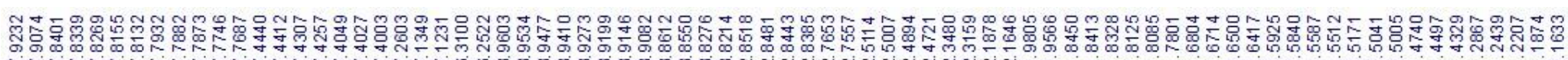
N

Scheme 2, 41
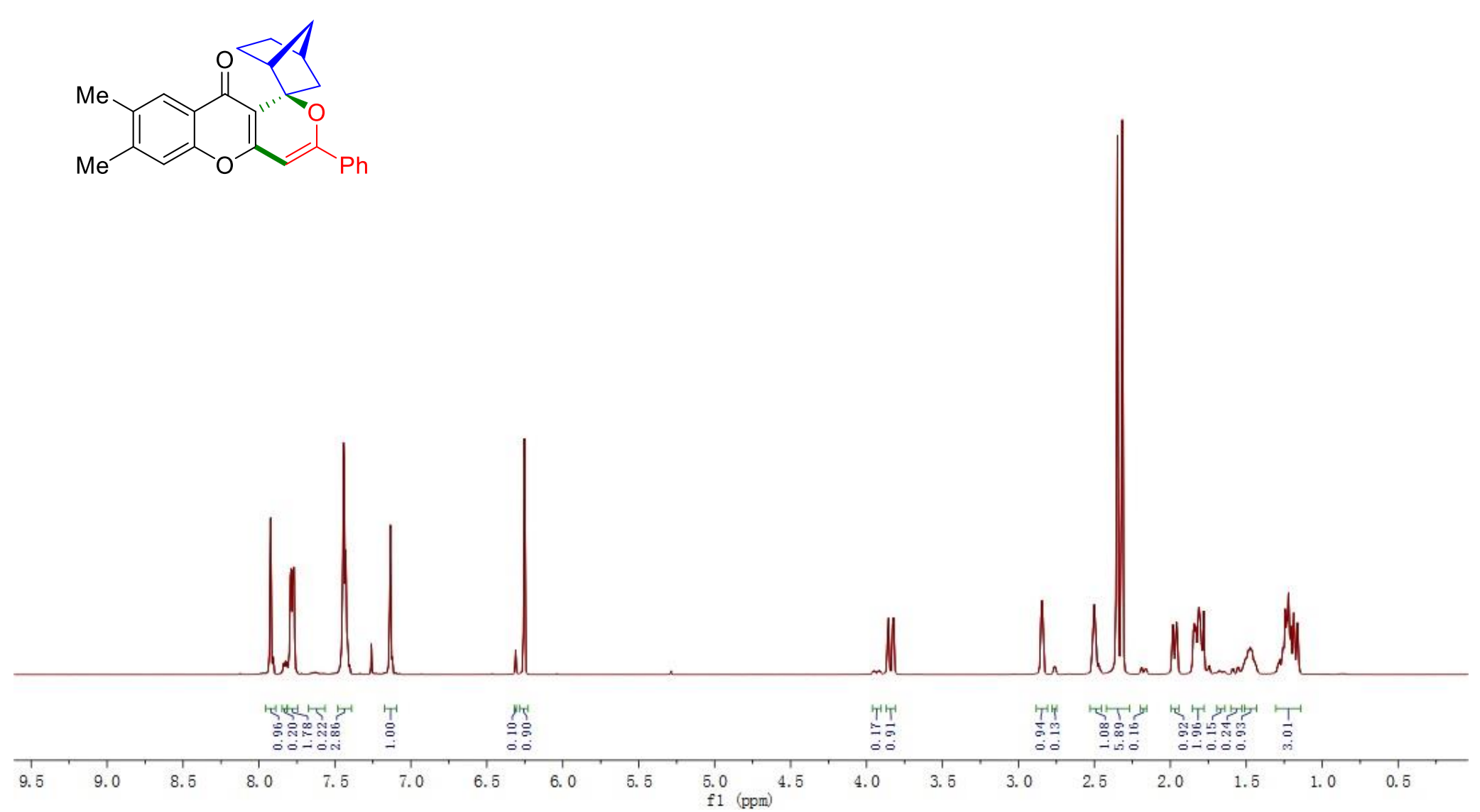


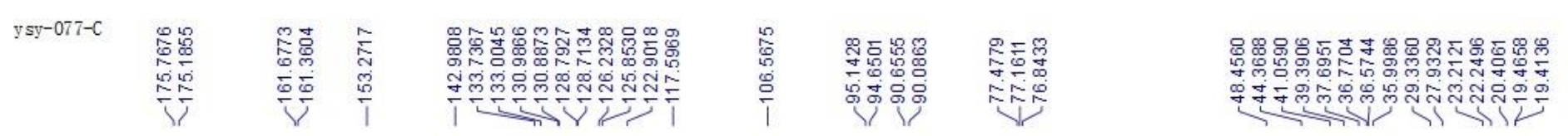

Scheme 2, 4I
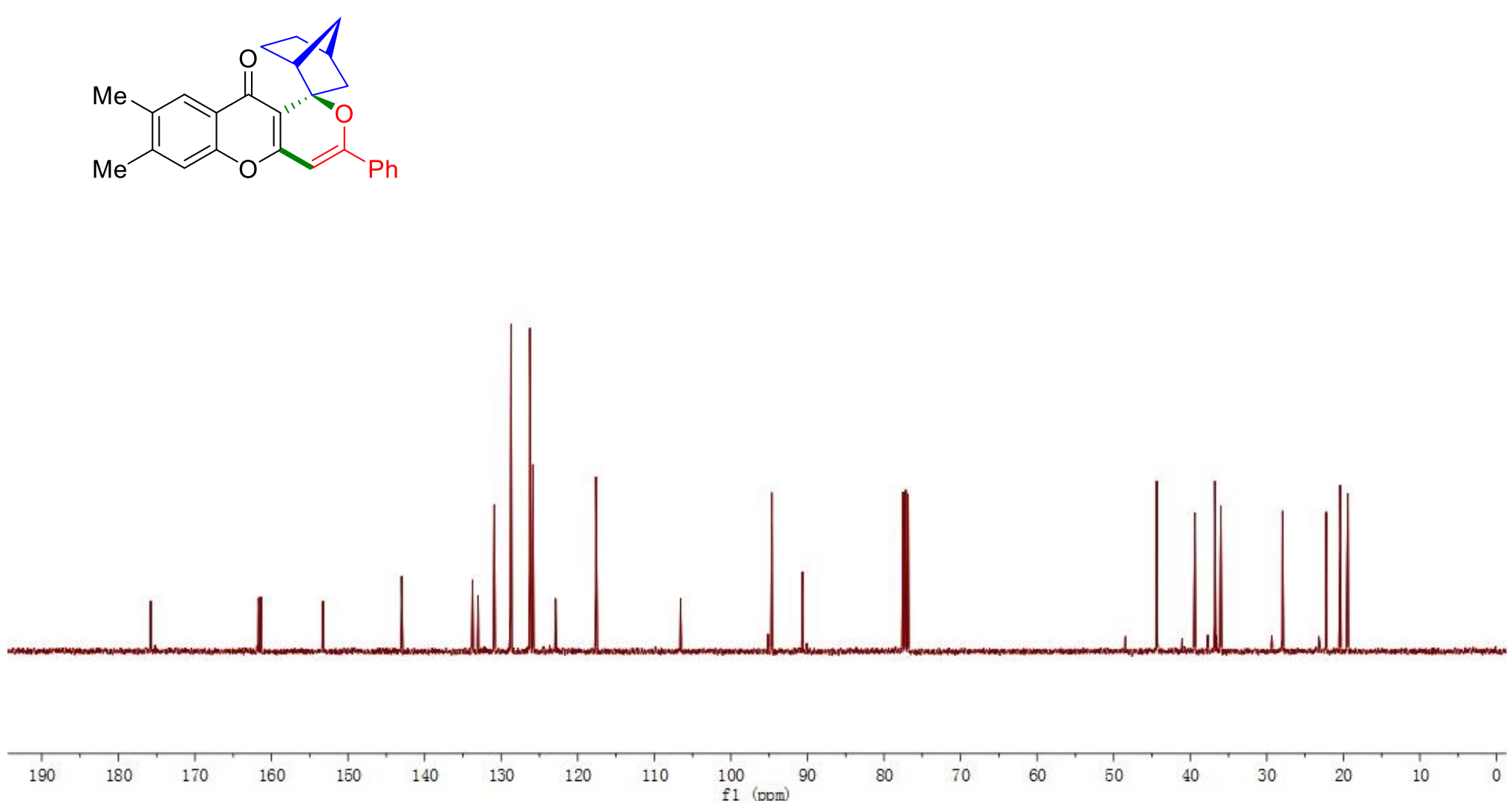
Scheme 2, 4m
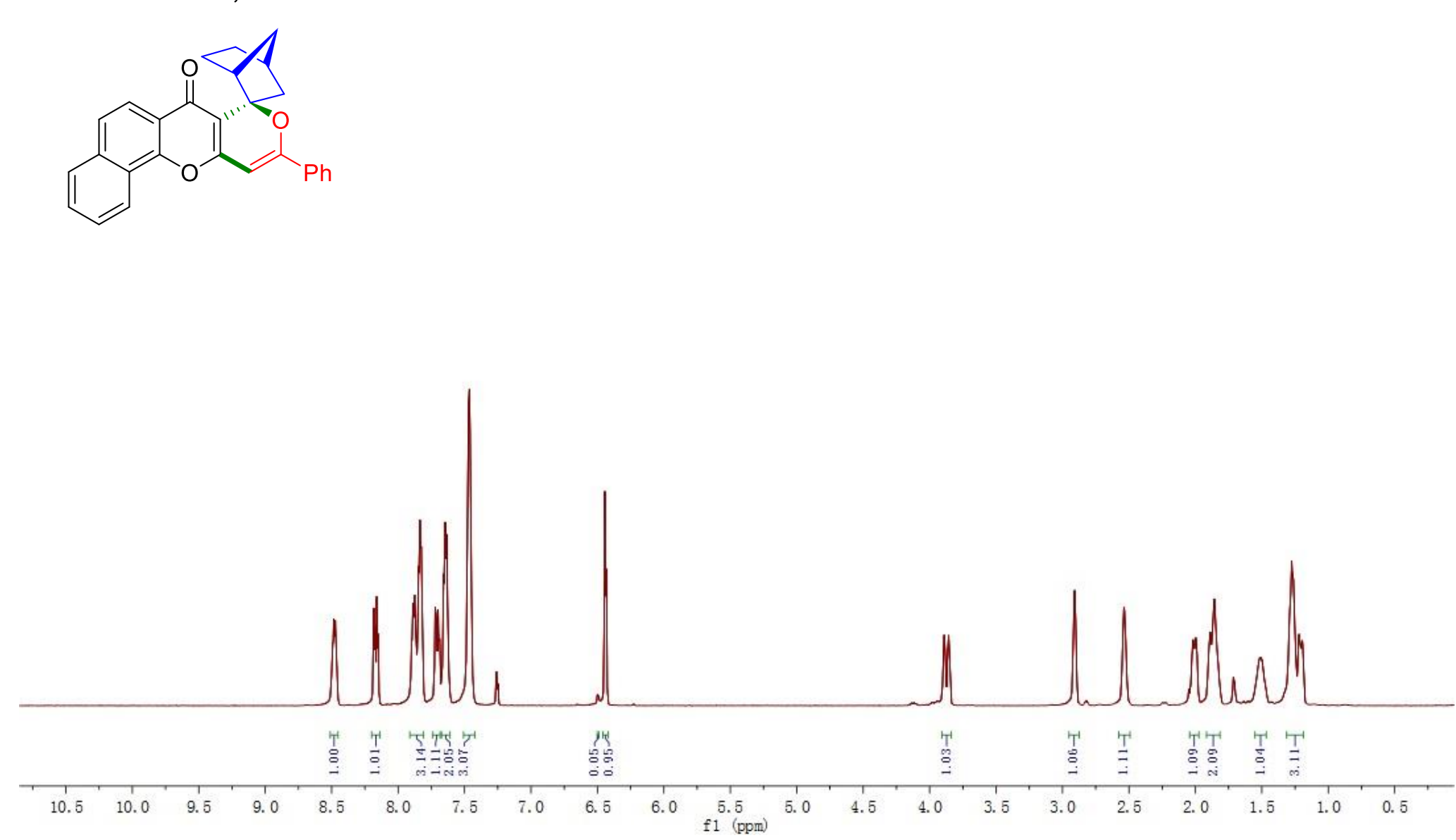

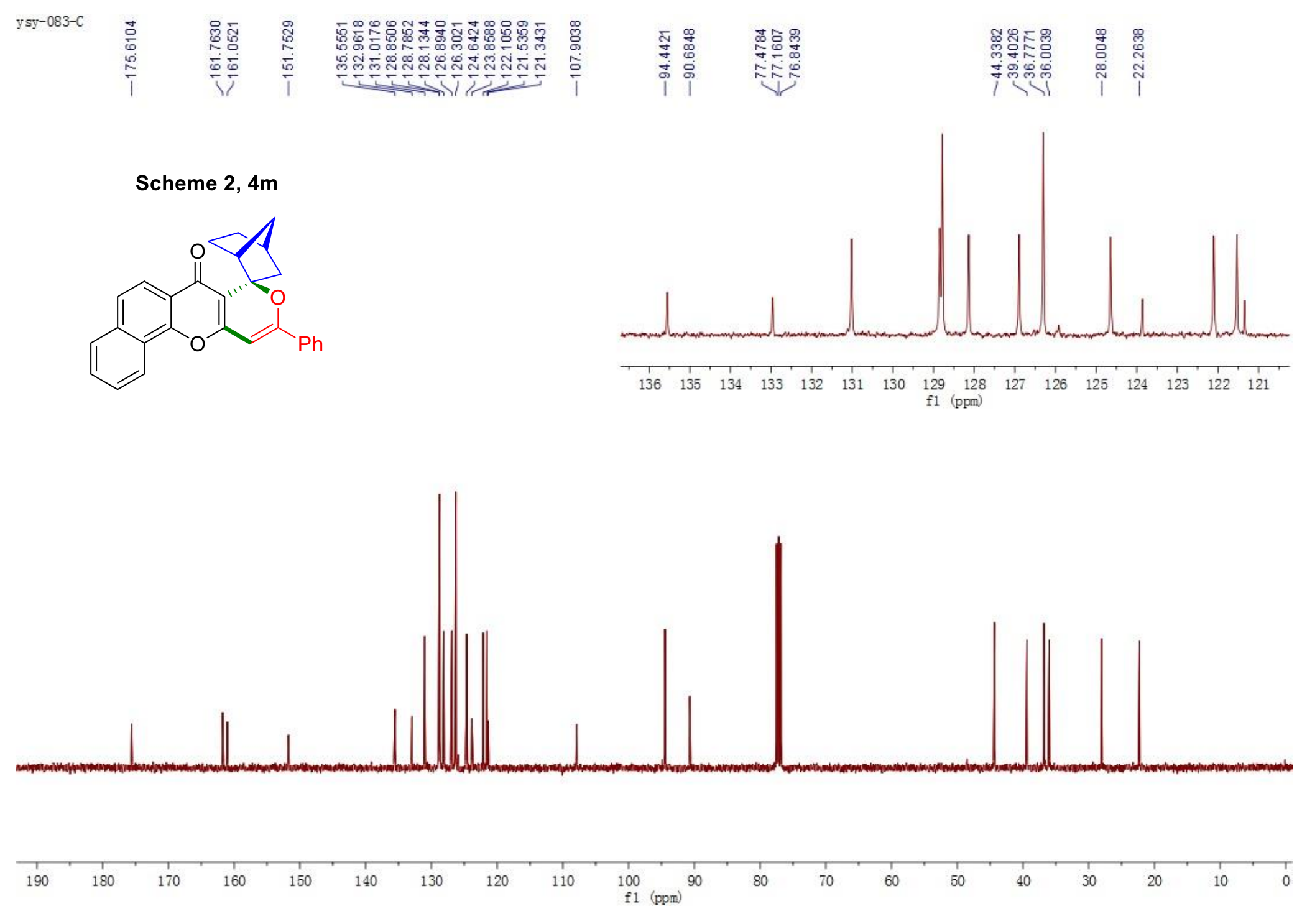
Scheme 2, 4n

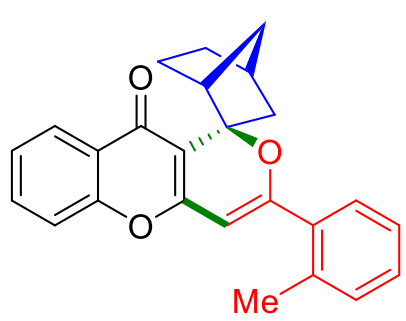

$\mathrm{Me}$

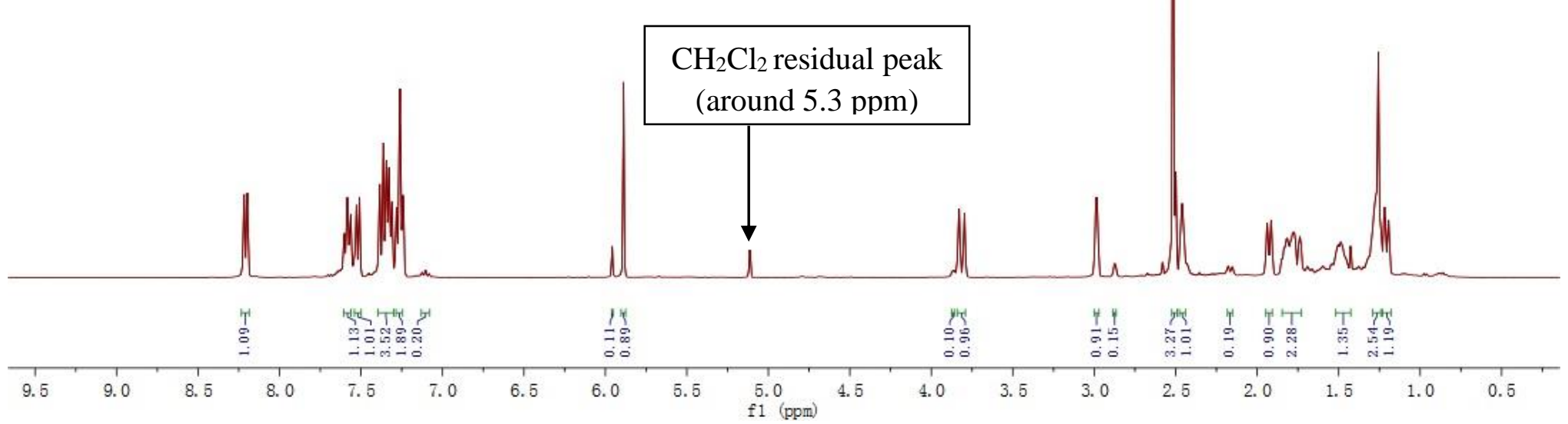




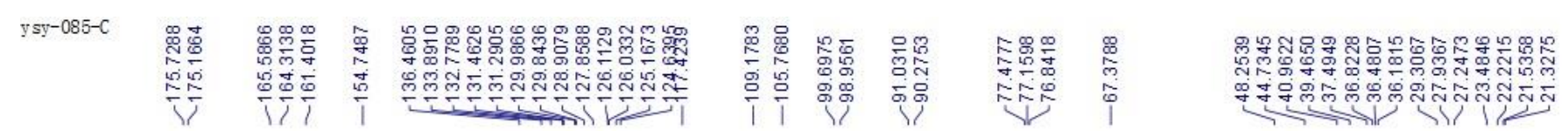

Scheme 2, 4n
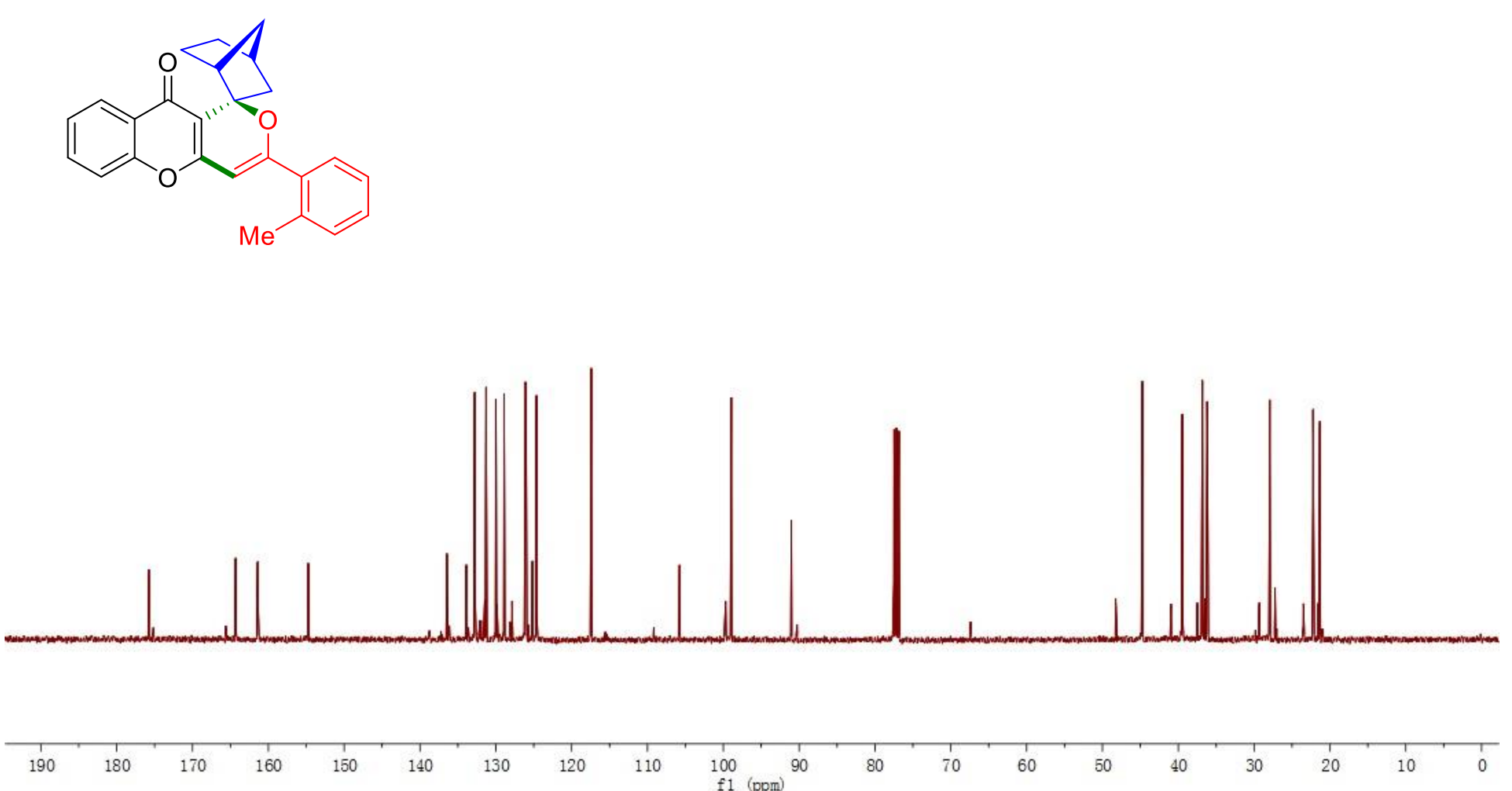
Scheme 2, 40

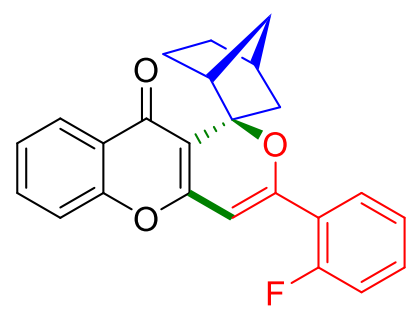

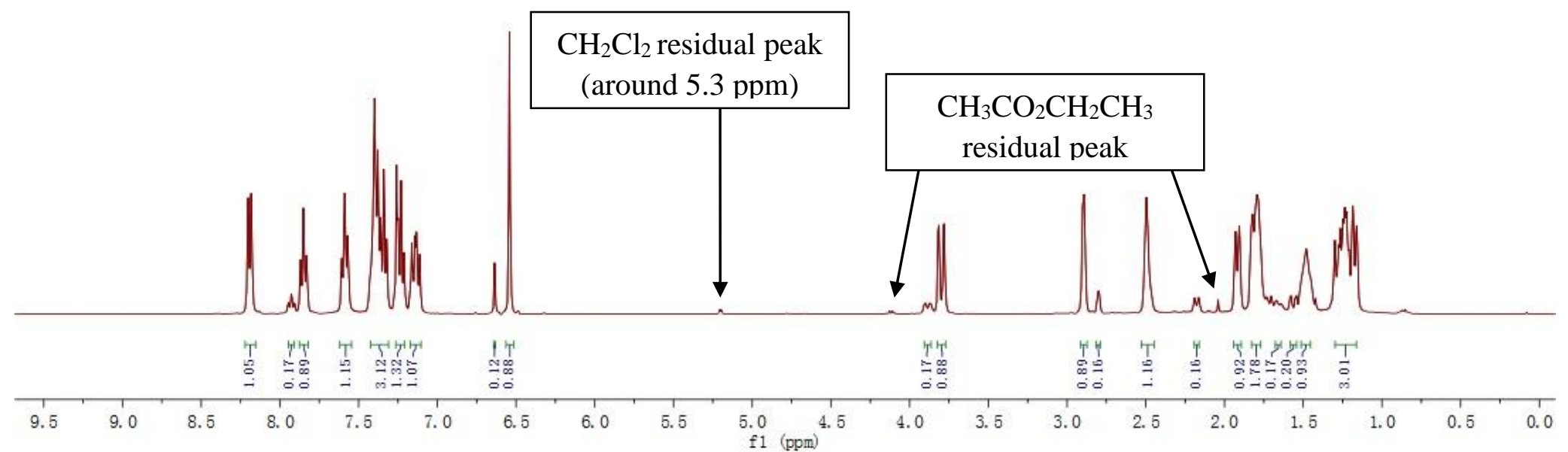



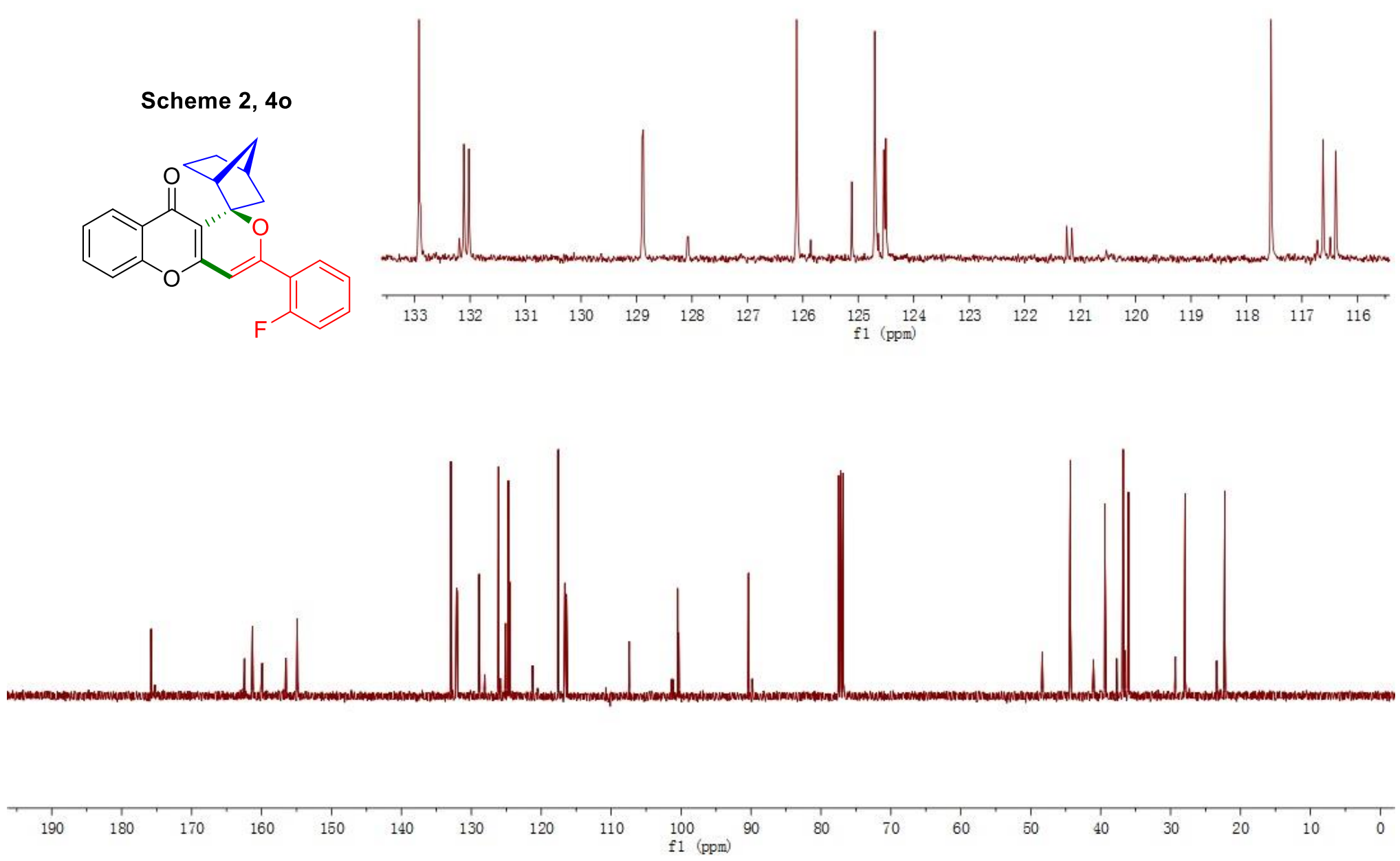
Scheme 2, 4p

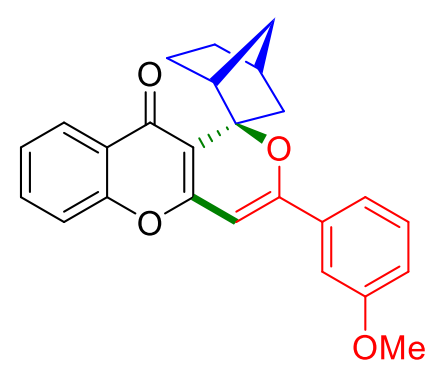

$\mathrm{CH}_{2} \mathrm{Cl}_{2}$ residual peak (around $5.3 \mathrm{ppm}$ )

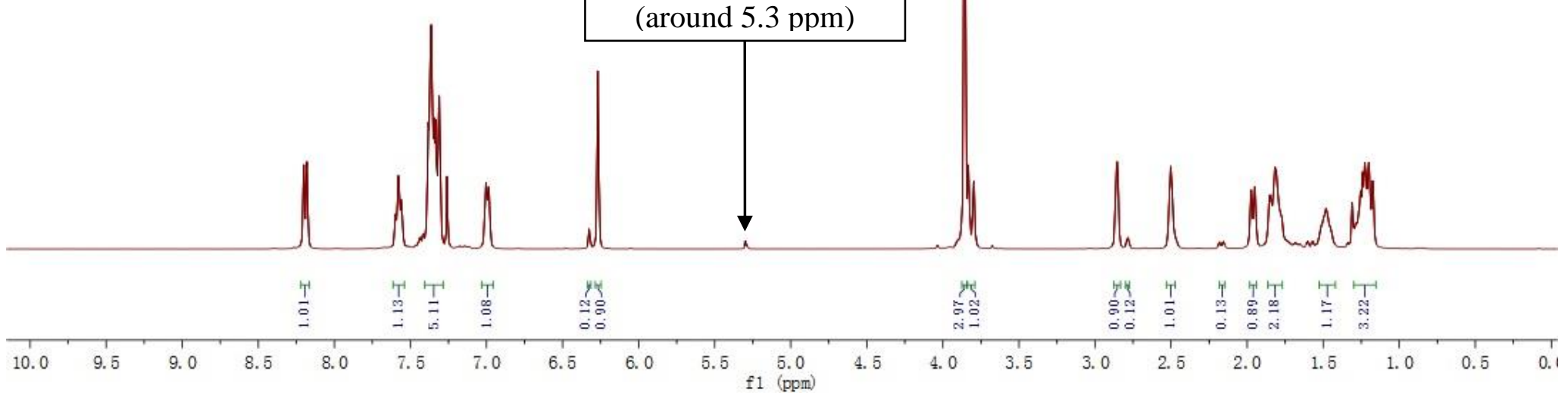


y $5 y-197-2-C$

Scheme 2, 4p
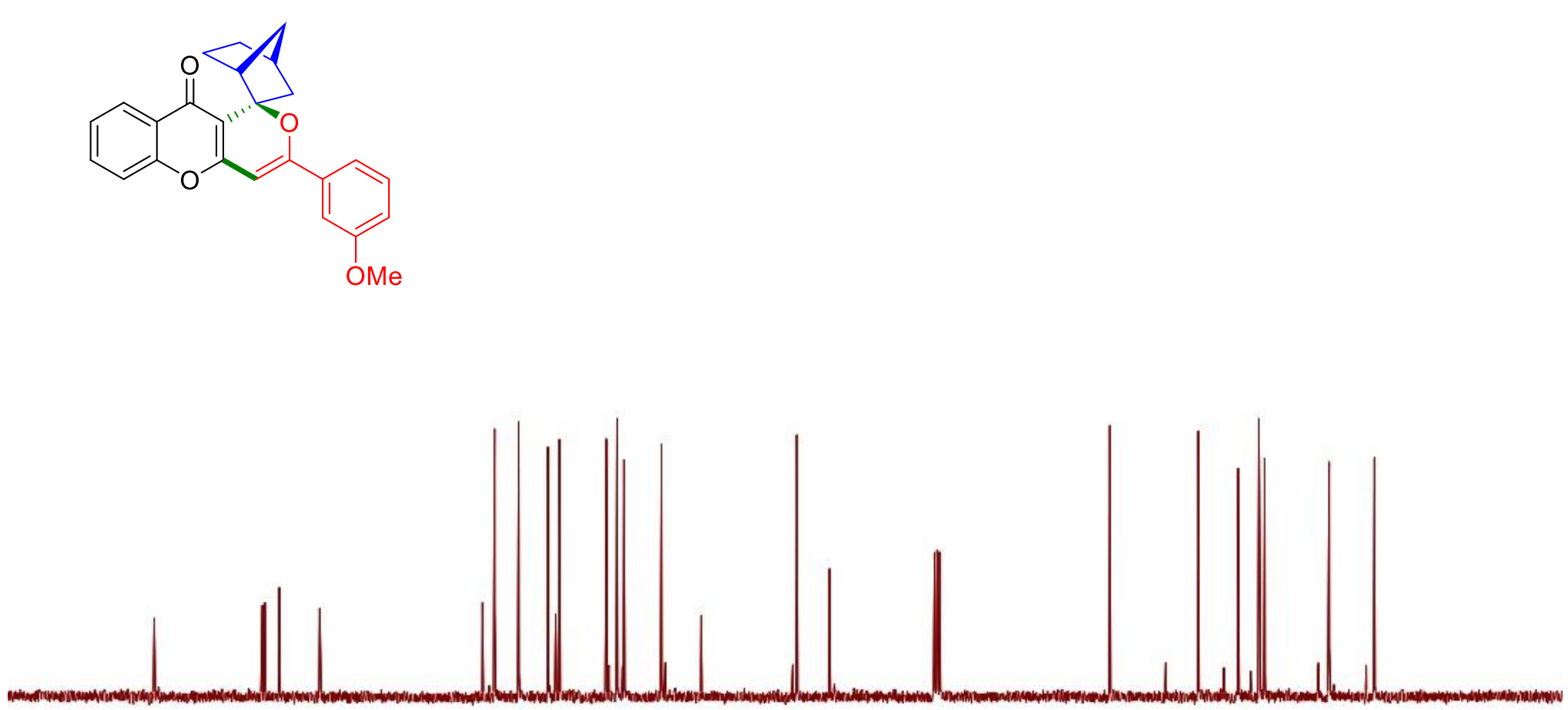


\section{Scheme 2, 4q}
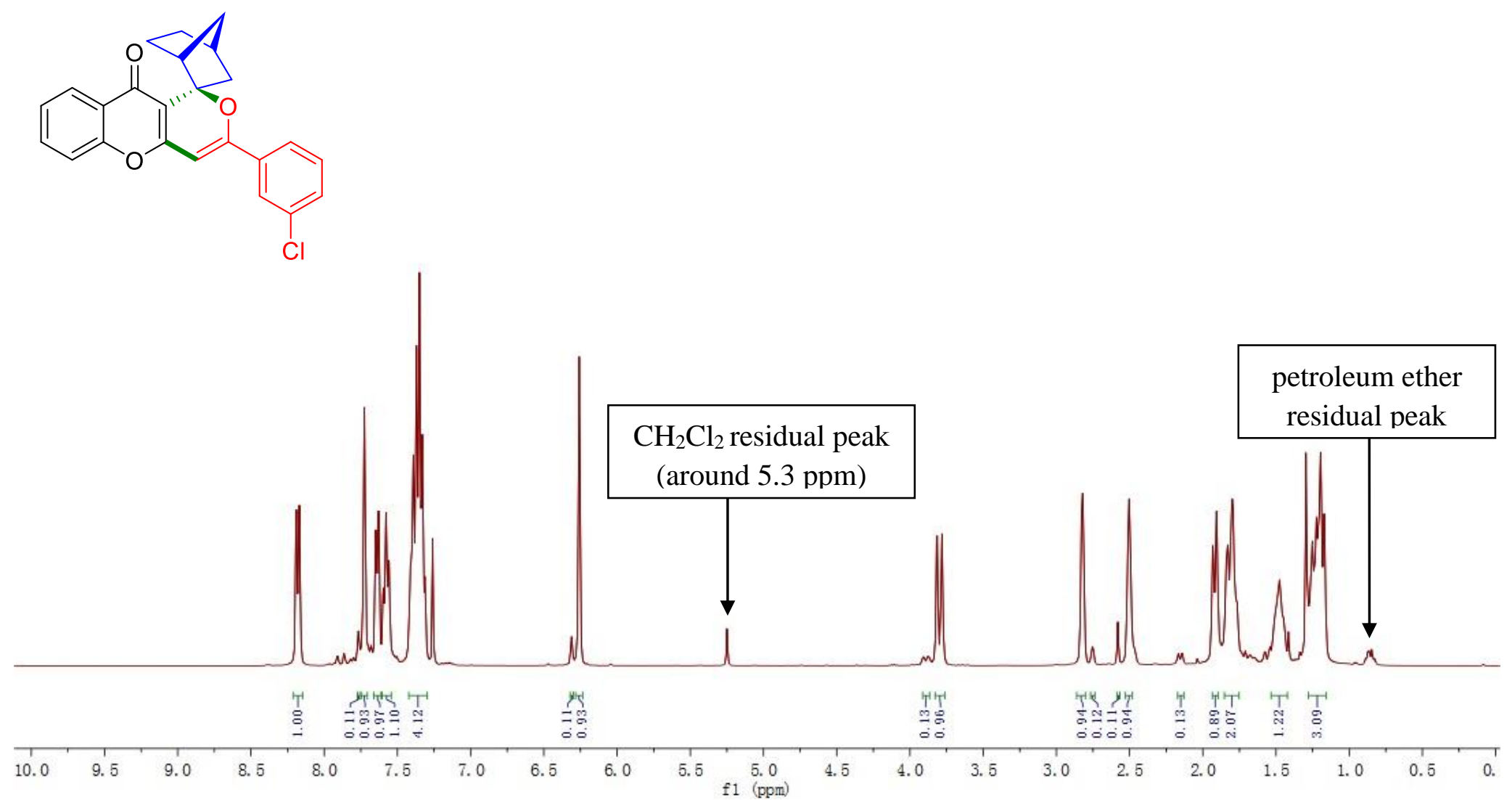
Scheme 2, 4q
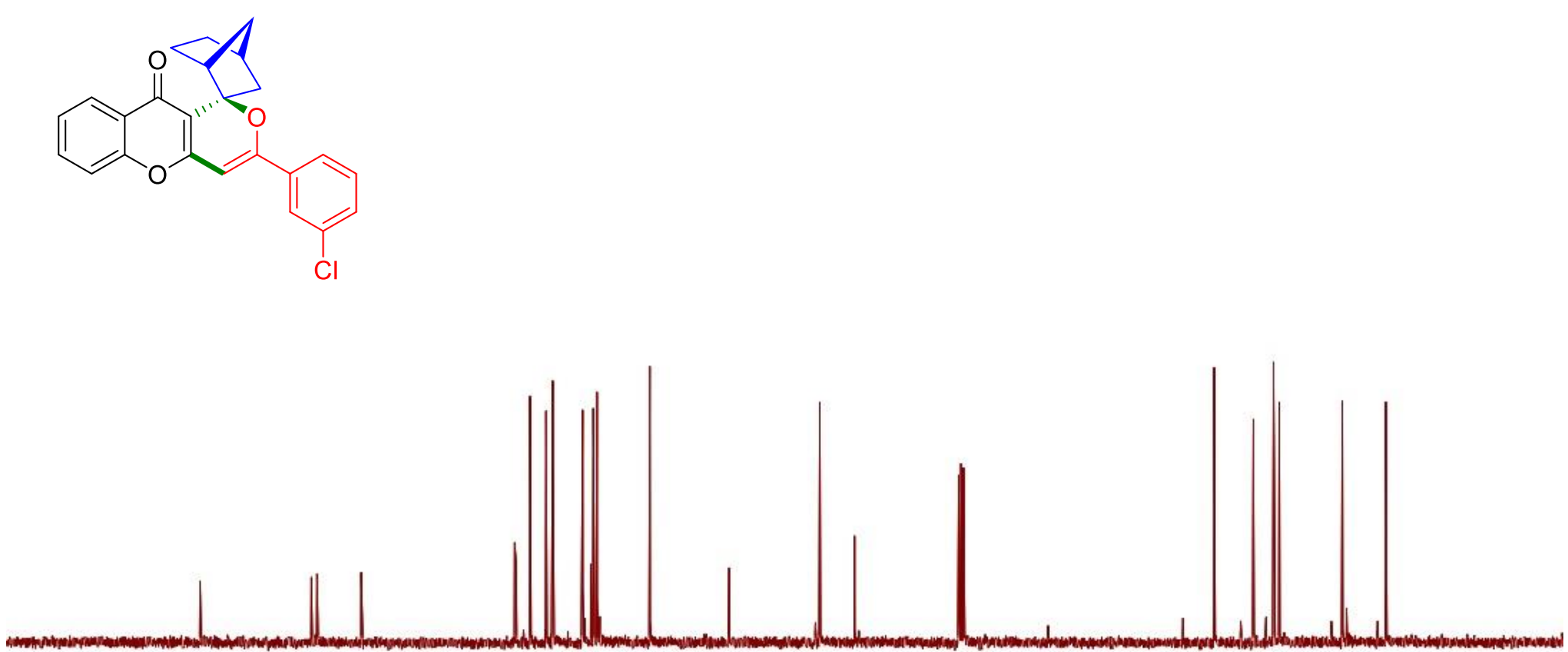

00
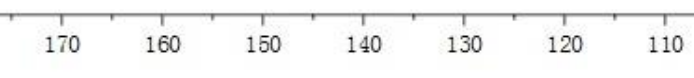

f1 100 
Scheme 2, 4r
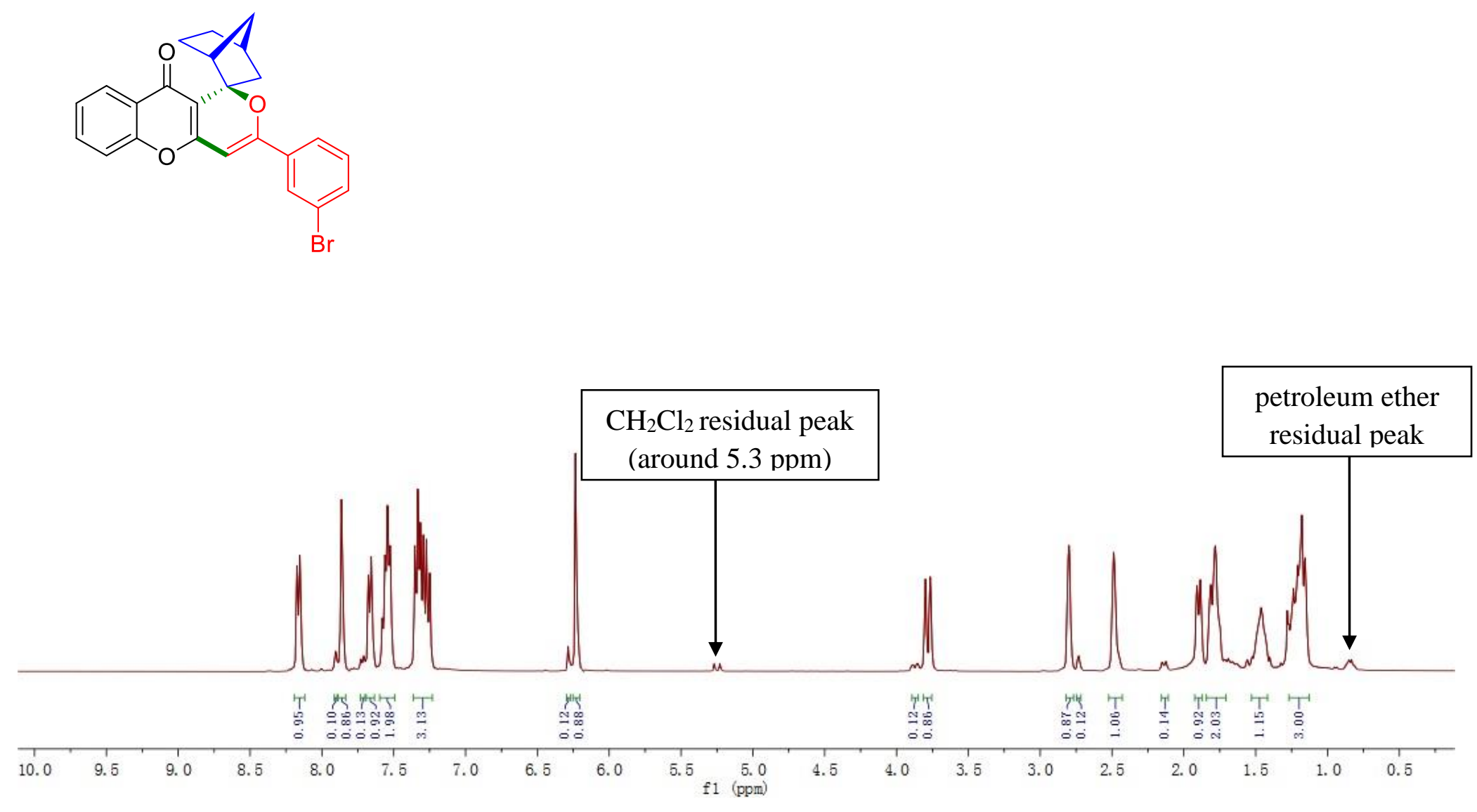
ysy-198-2-C

Scheme 2, 4r
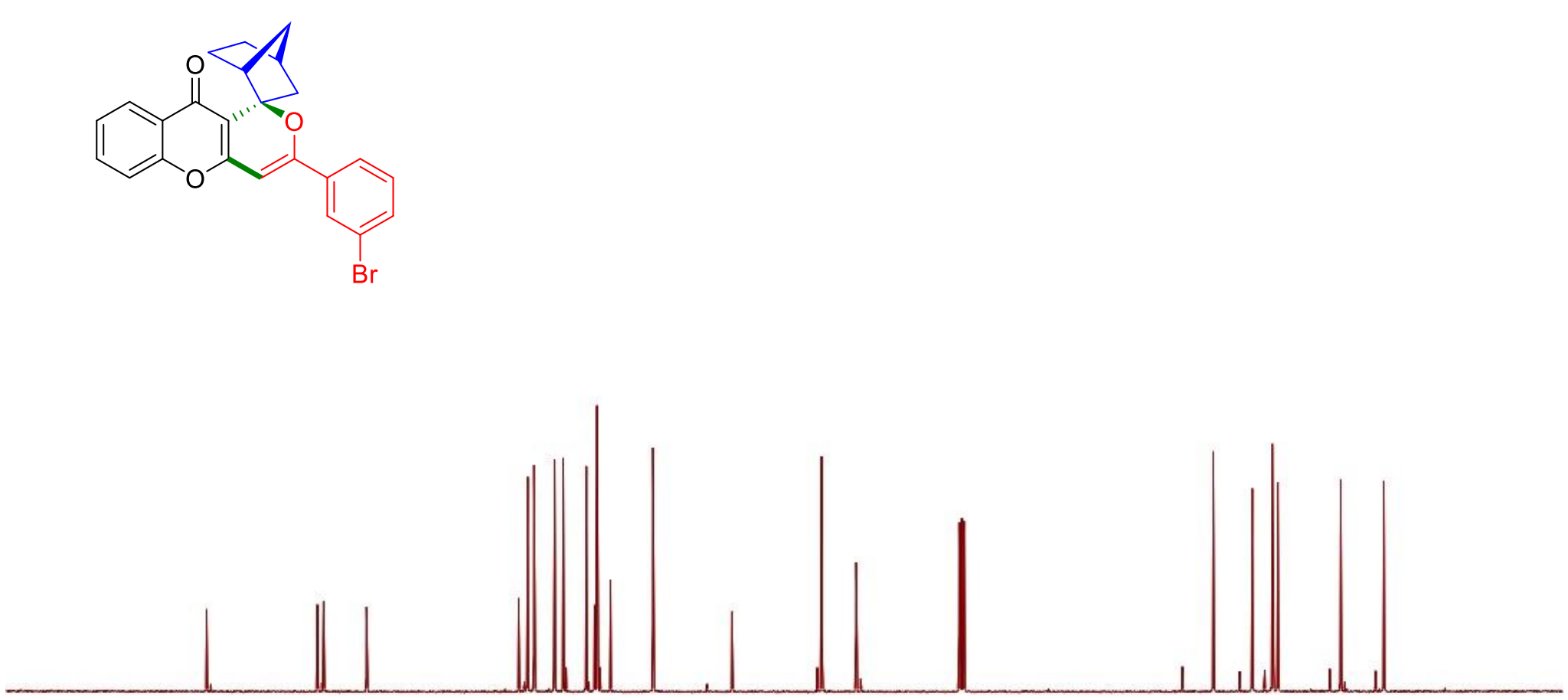
Scheme 2, 4s
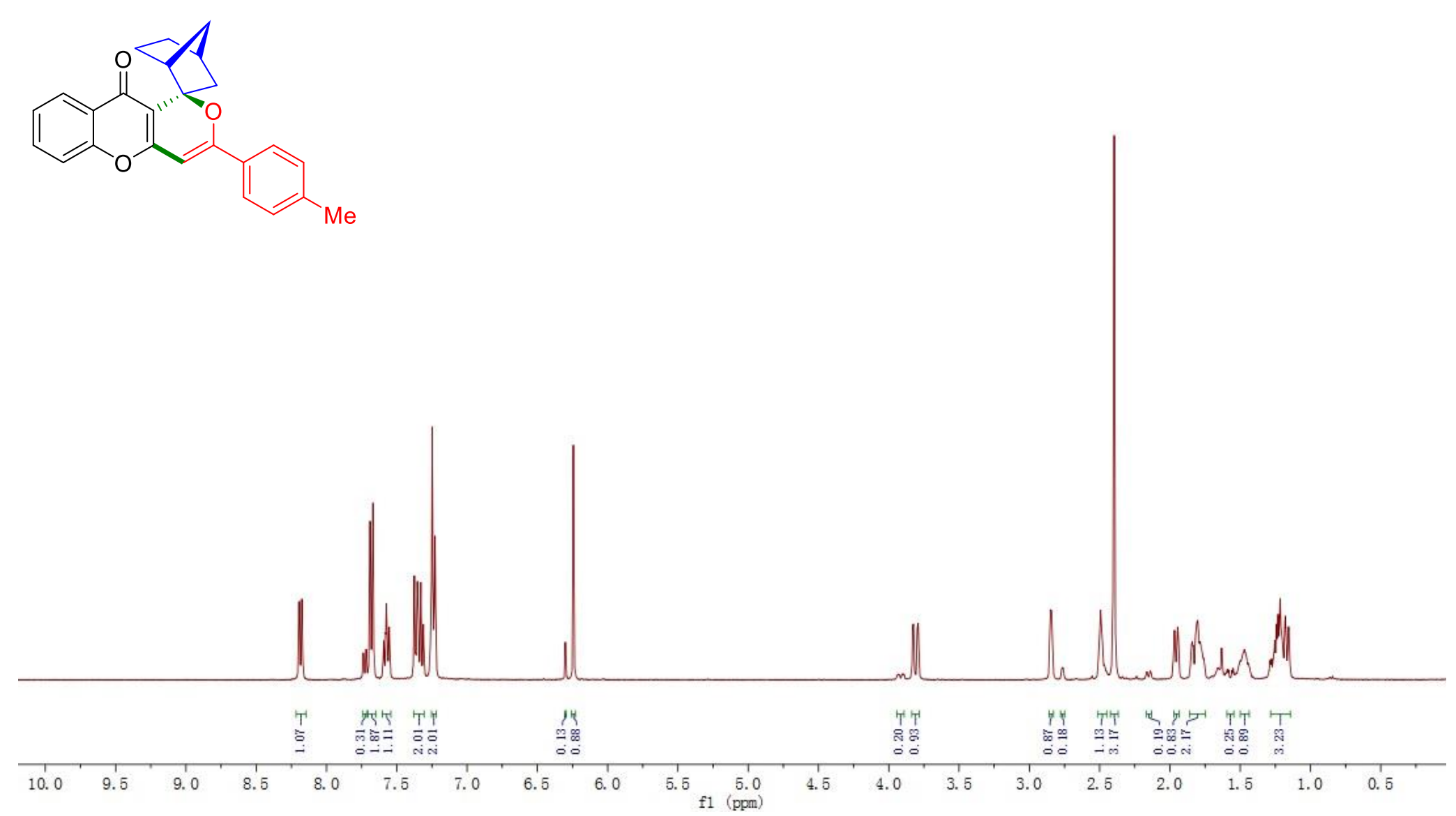
Scheme 2, 4s<smiles>Cc1ccc(C2=Cc3oc4ccccc4c(=O)c3[C@H]3CC4CC(C2)C(C4)C3)cc1</smiles>

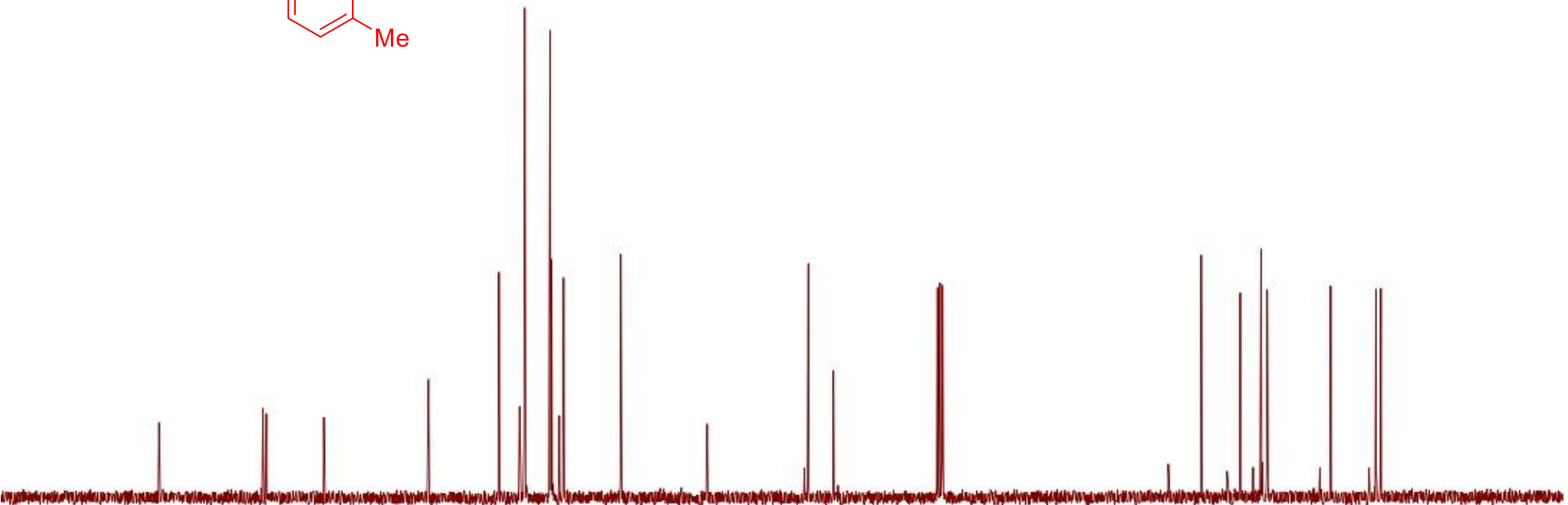


Scheme 2, 4t
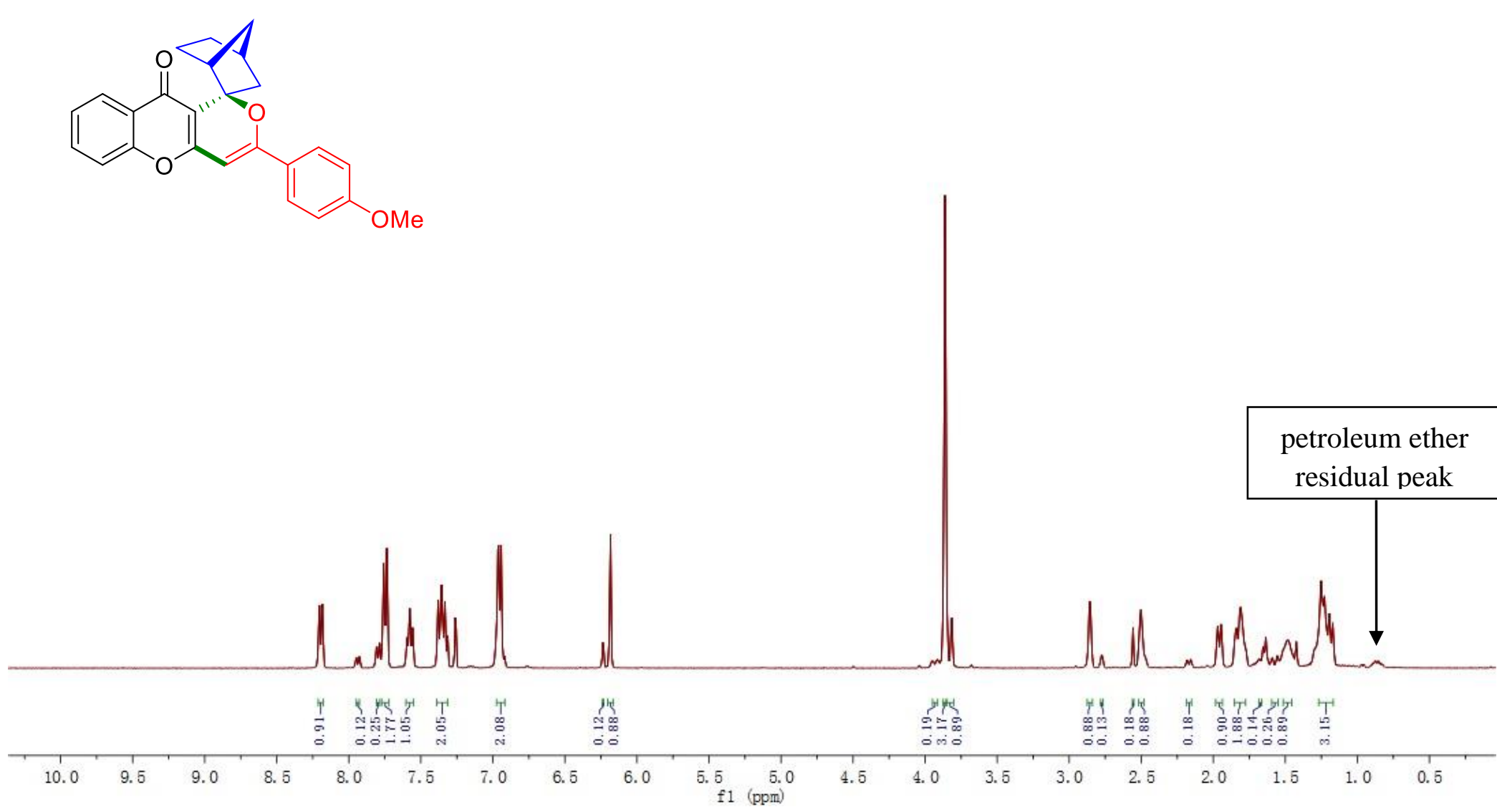


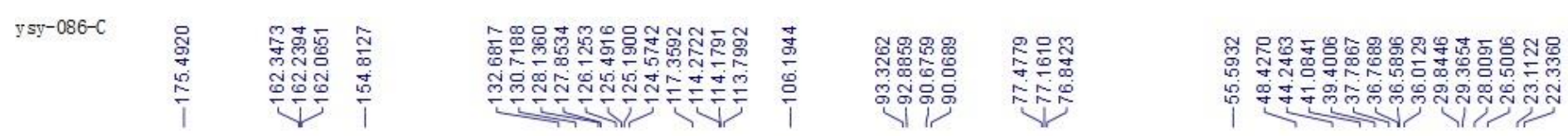

Scheme 2, 4t

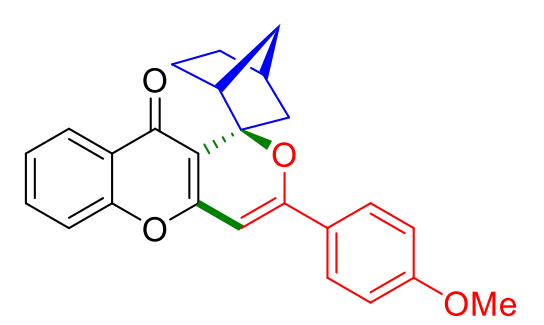

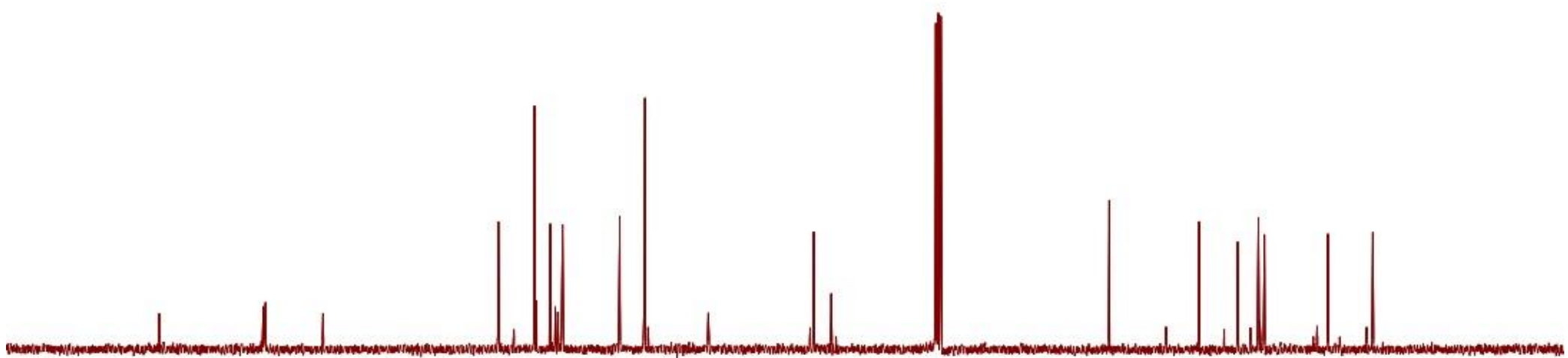

190

$180 \quad 170$

$160 \quad 150$

$140 \quad 130$

$120 \quad 110$

$100 \quad 90$

$80 \quad 70$

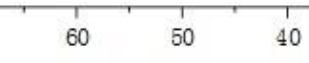

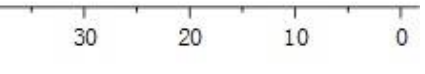


Scheme 2, 4u
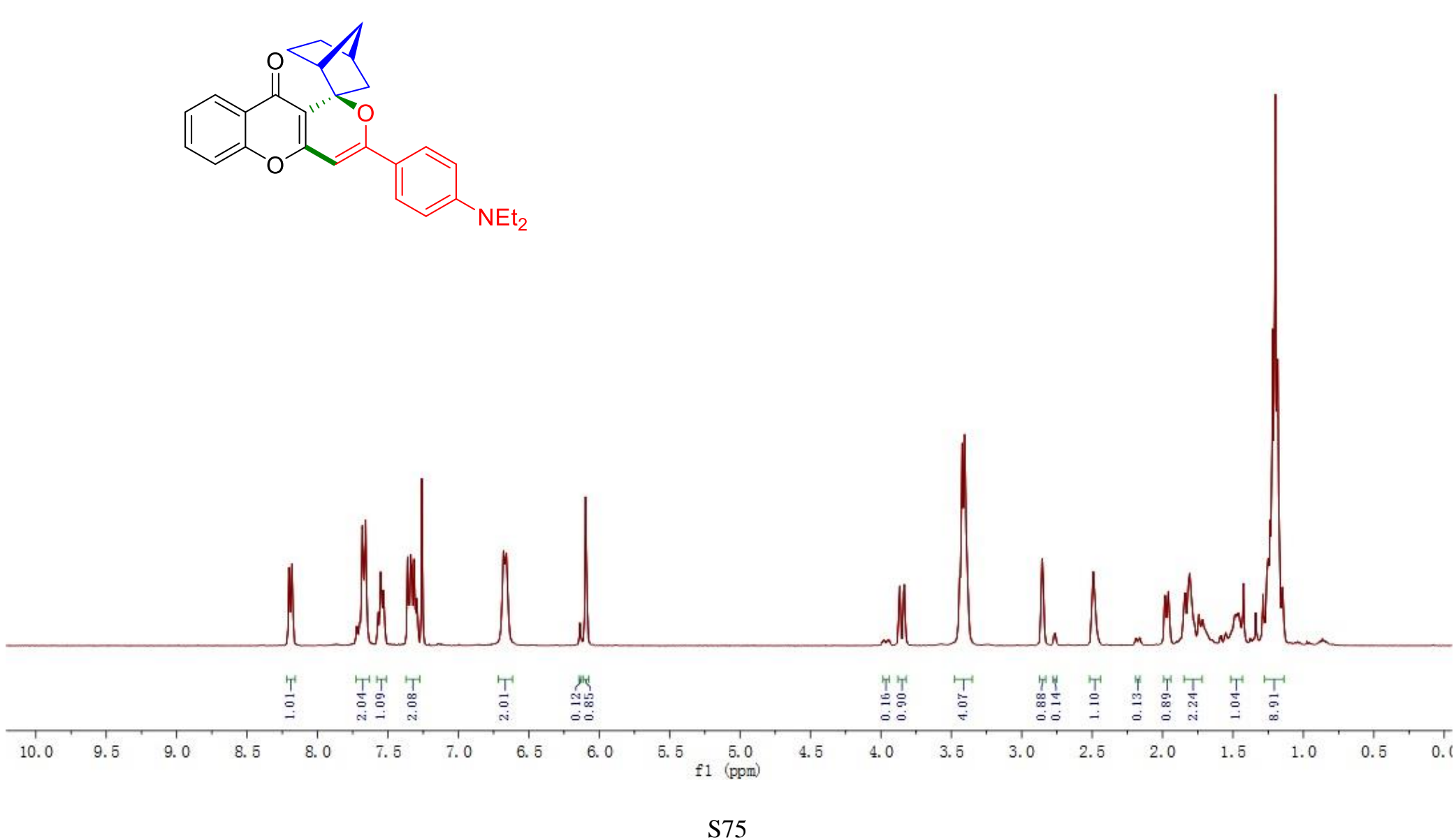


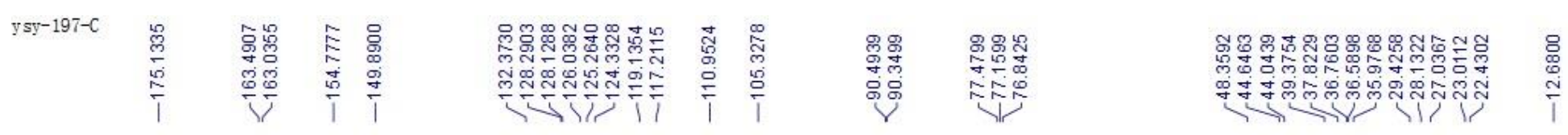

Scheme 2, 4u

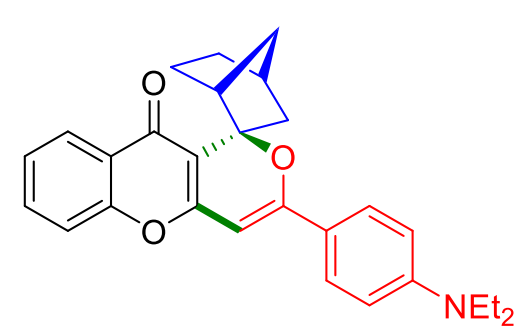

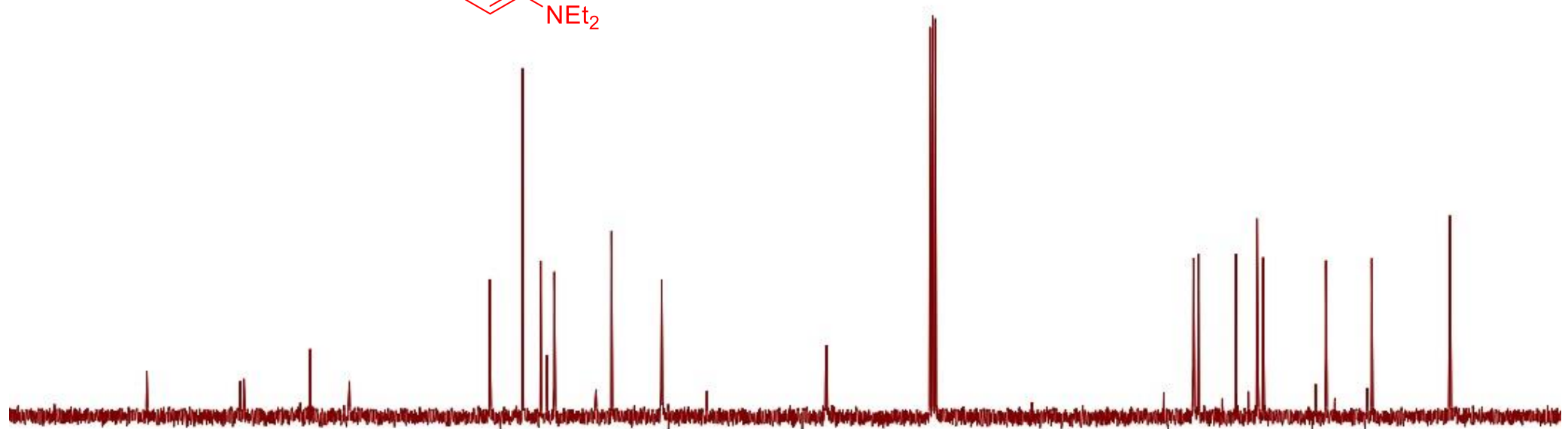

190

180

$170 \quad 160$

$150 \quad 140$

$130 \quad 120$

110

${ }^{100}{ }^{(p p m)} 90$

80

70

50

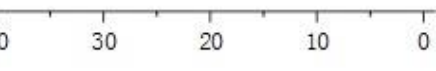




\section{Scheme 2, 4v}
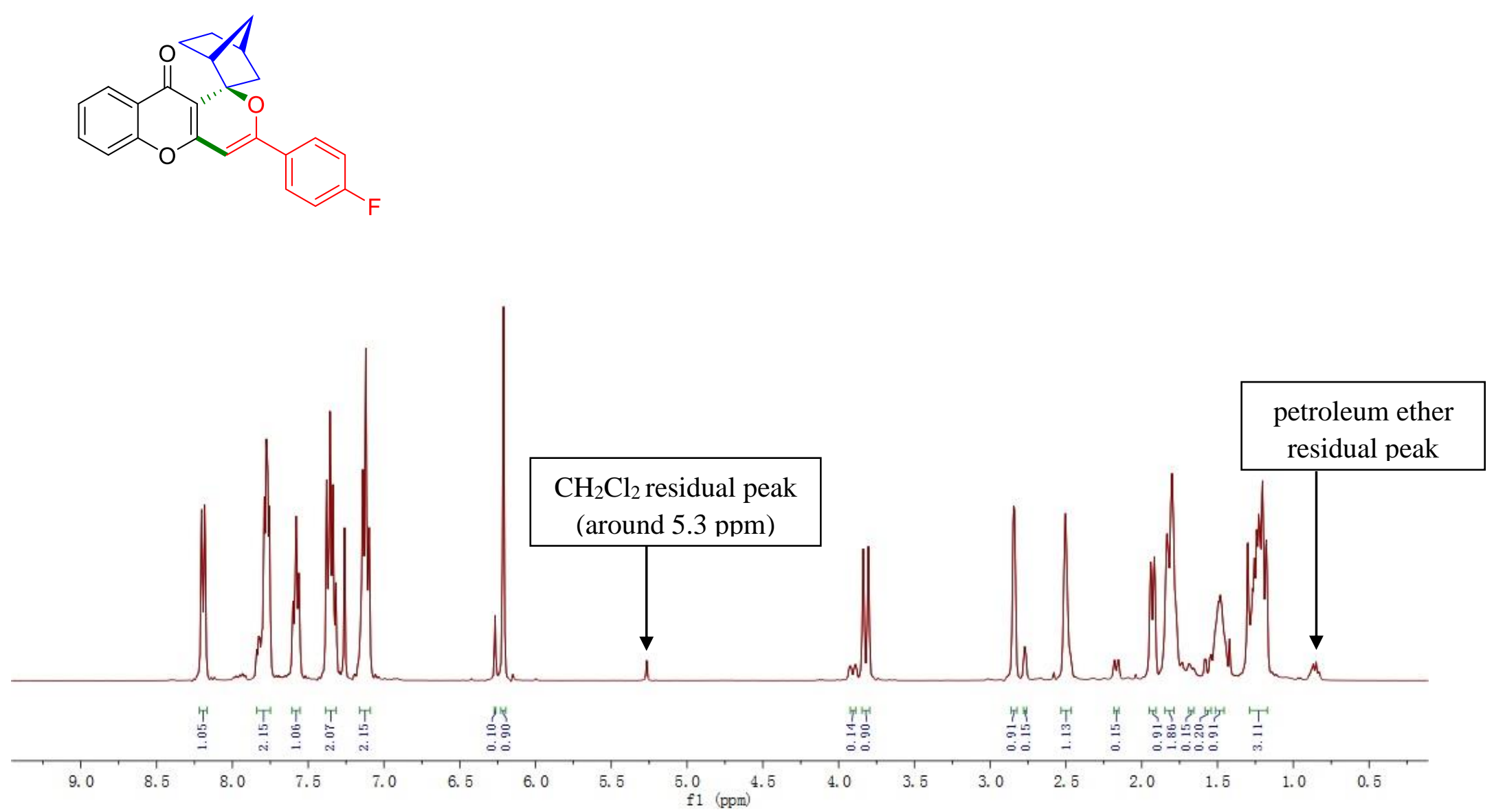

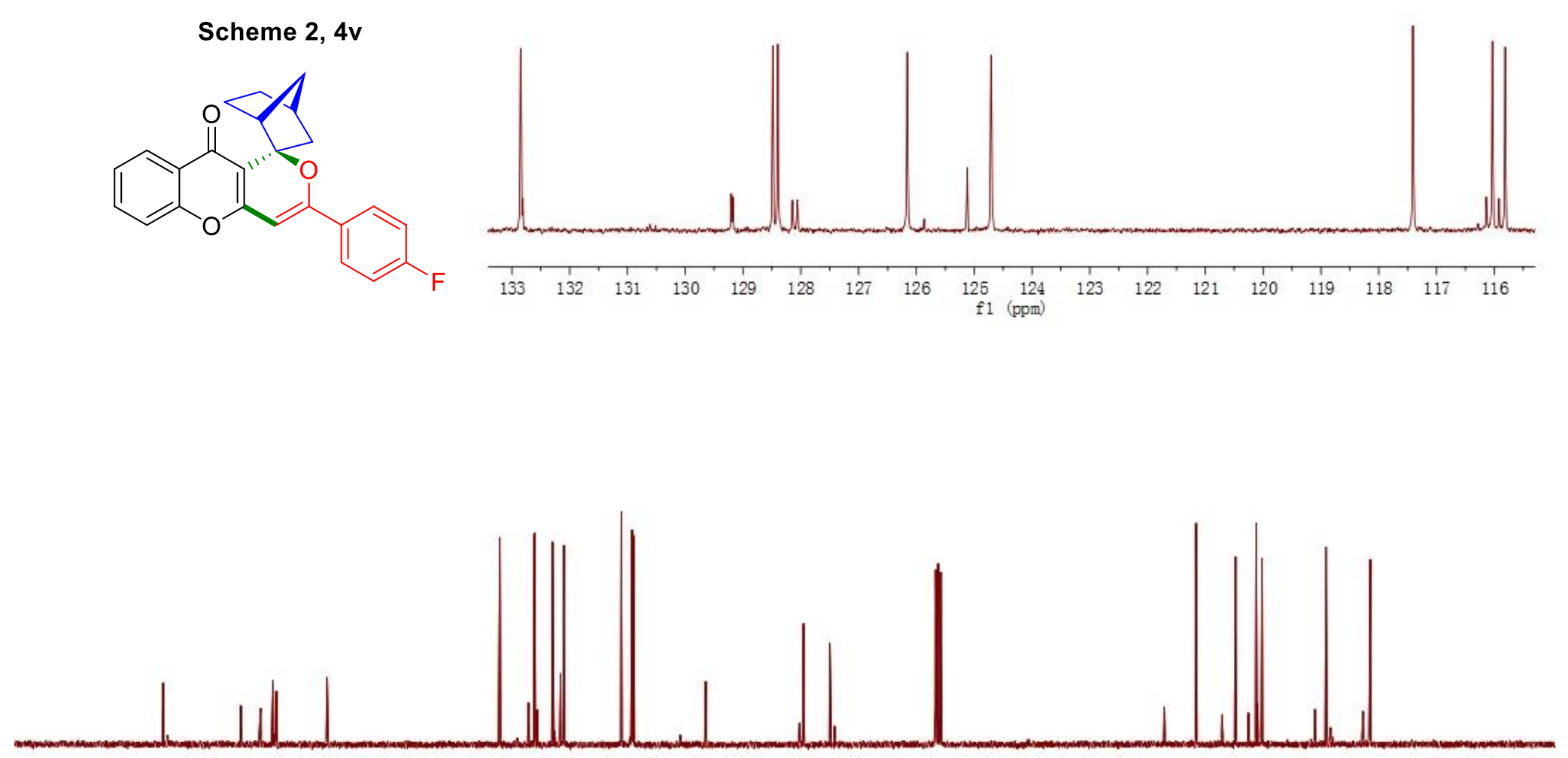
Scheme 2, 4w
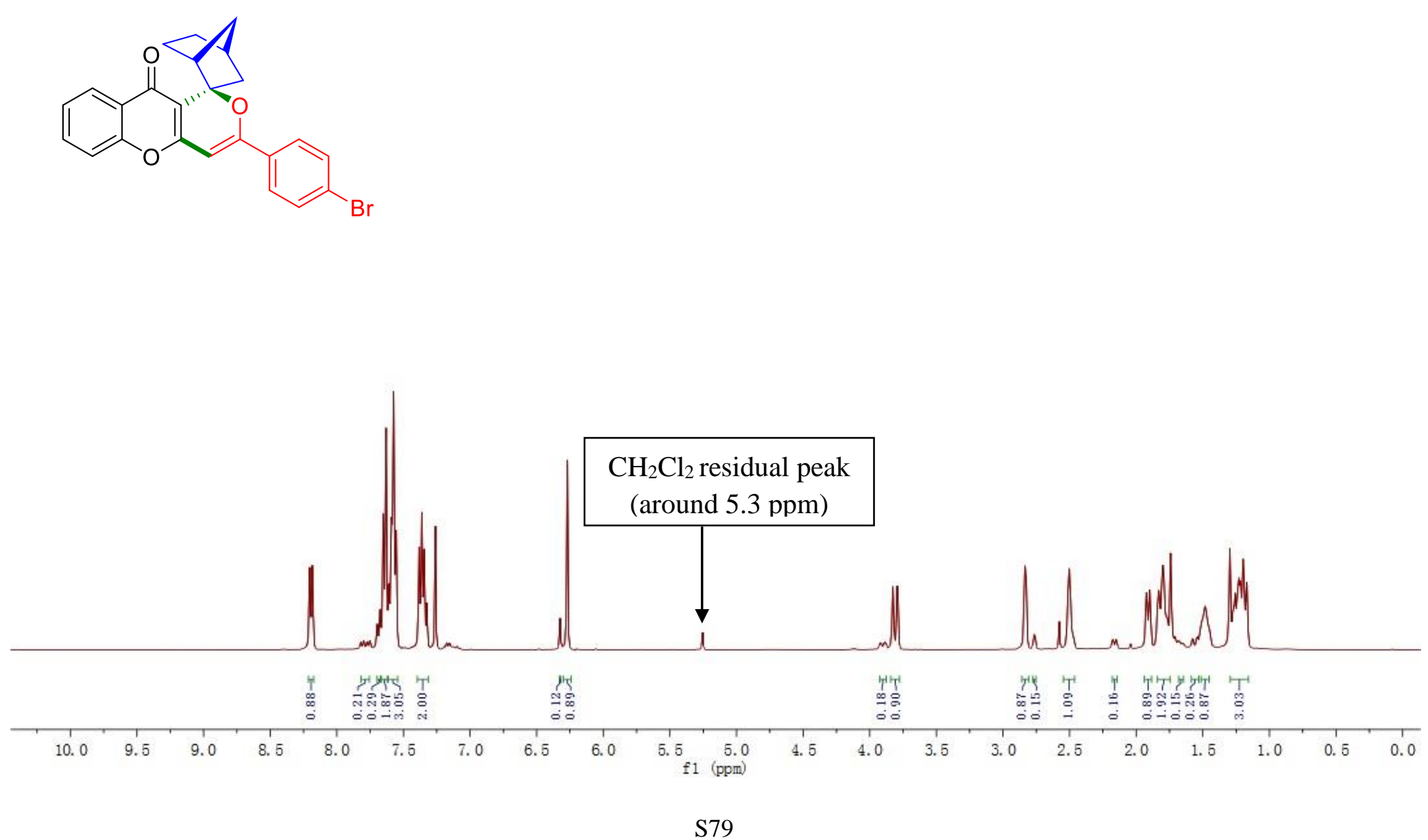


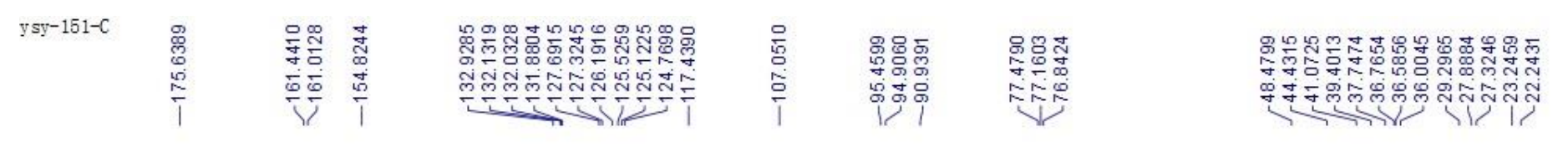

Scheme 2, 4w

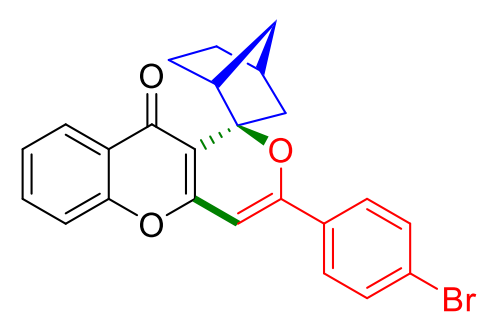

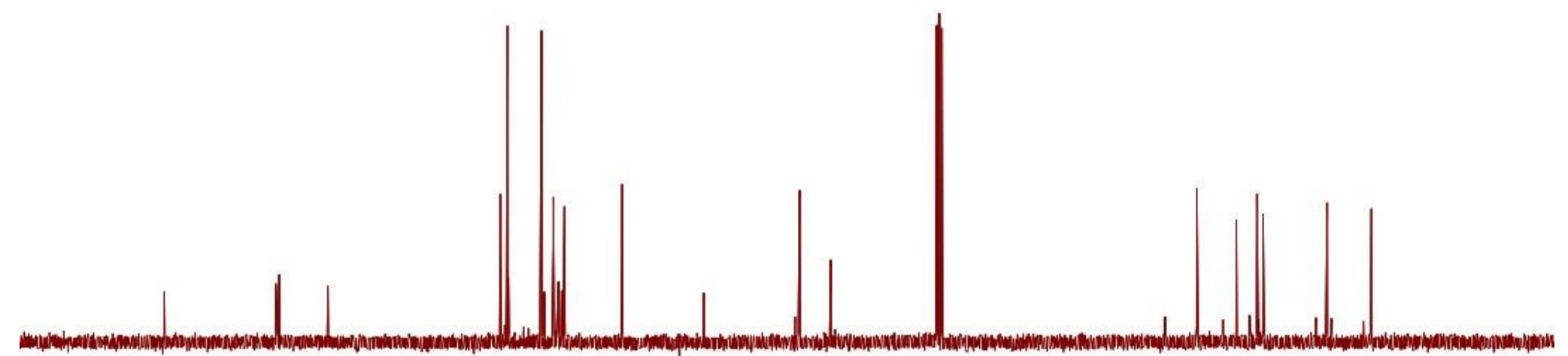

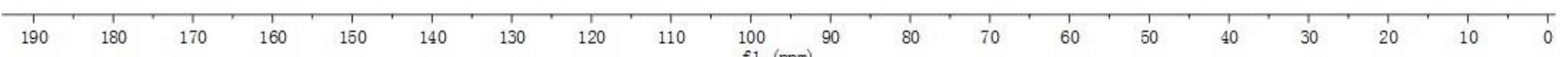


Scheme 2, 4x
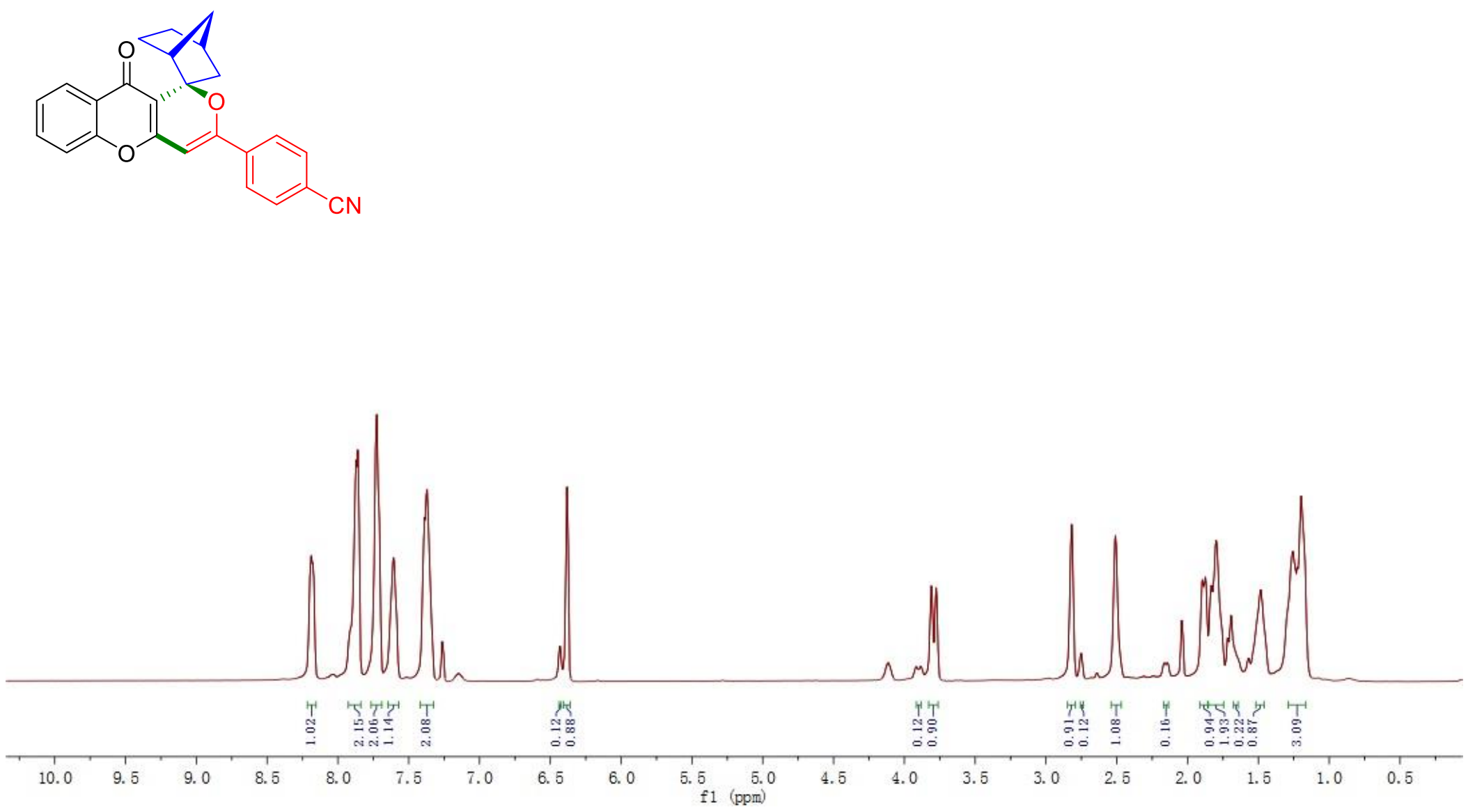
Scheme 2, 4x
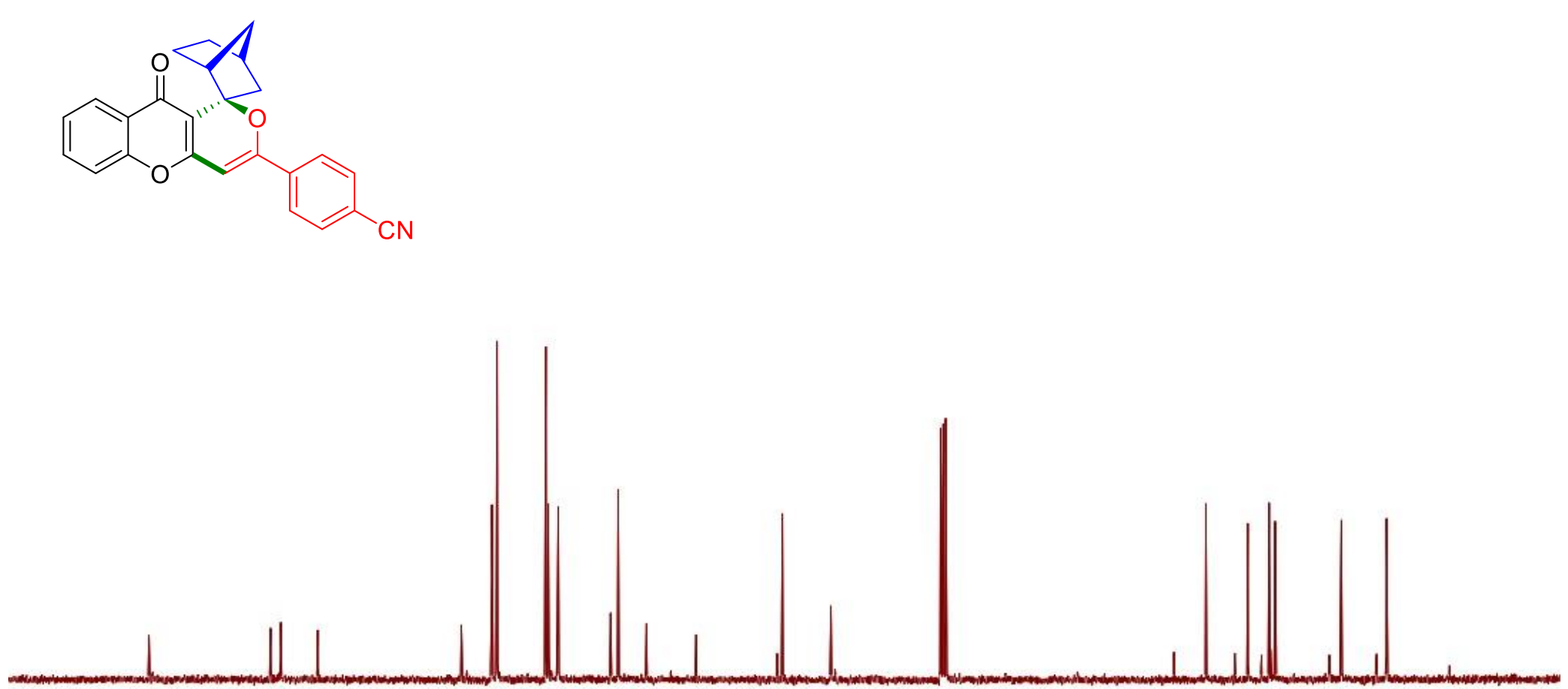

$190 \quad 180 \quad 170 \quad 160 \quad 150$

$140 \quad 130$

$120 \quad 110$ $100 \quad 90$

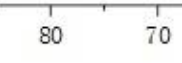

60

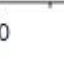


Scheme 2, 4y
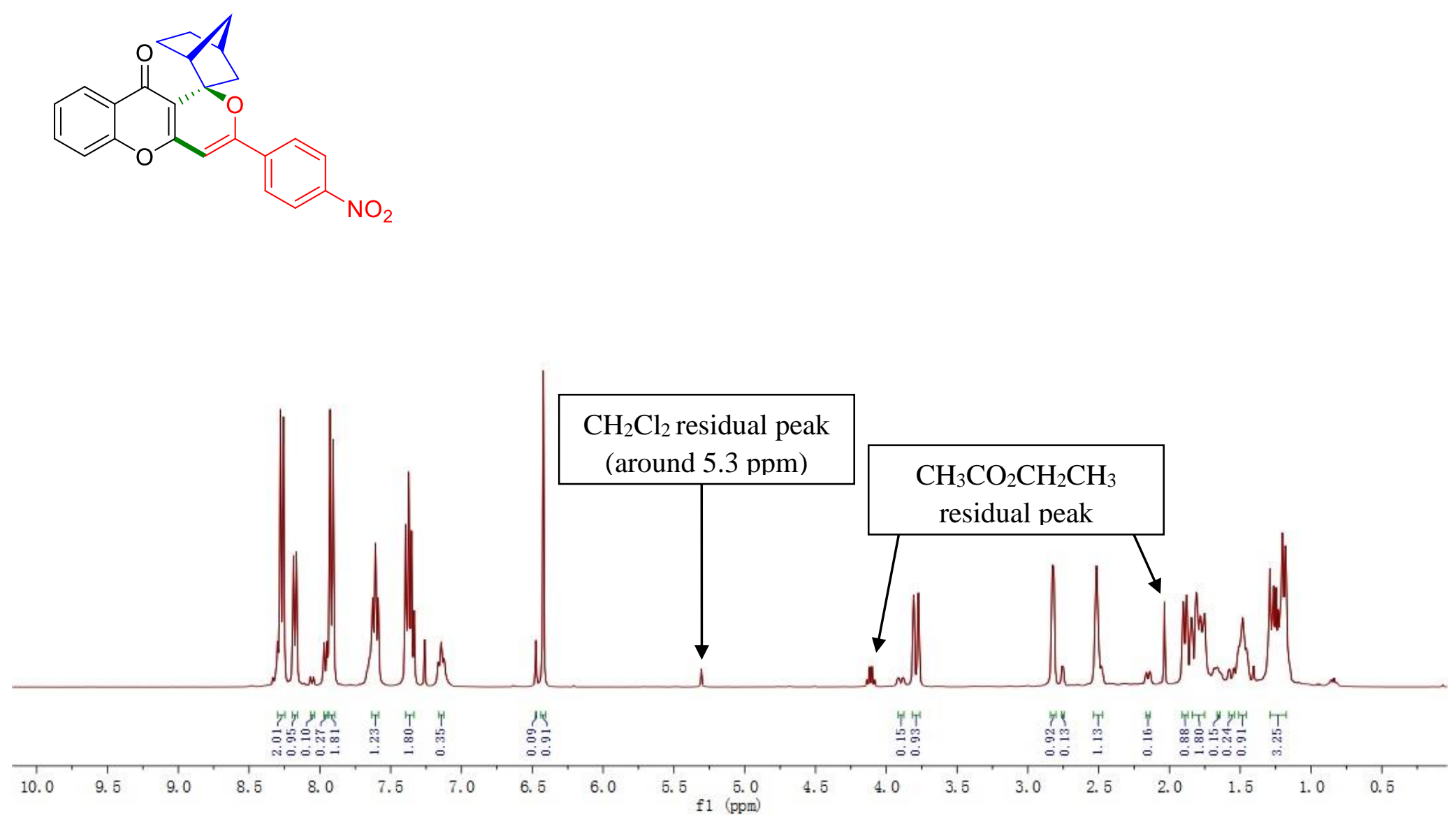


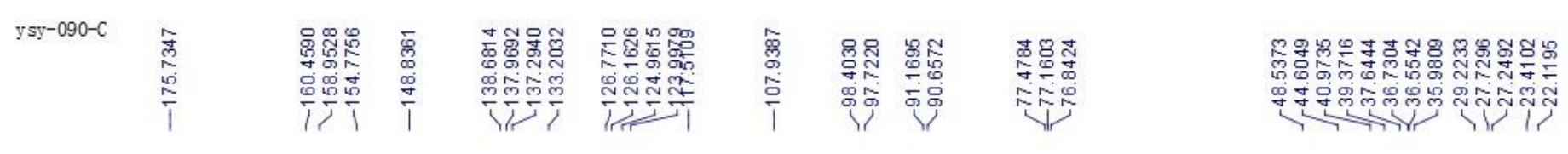

Scheme 2, 4y
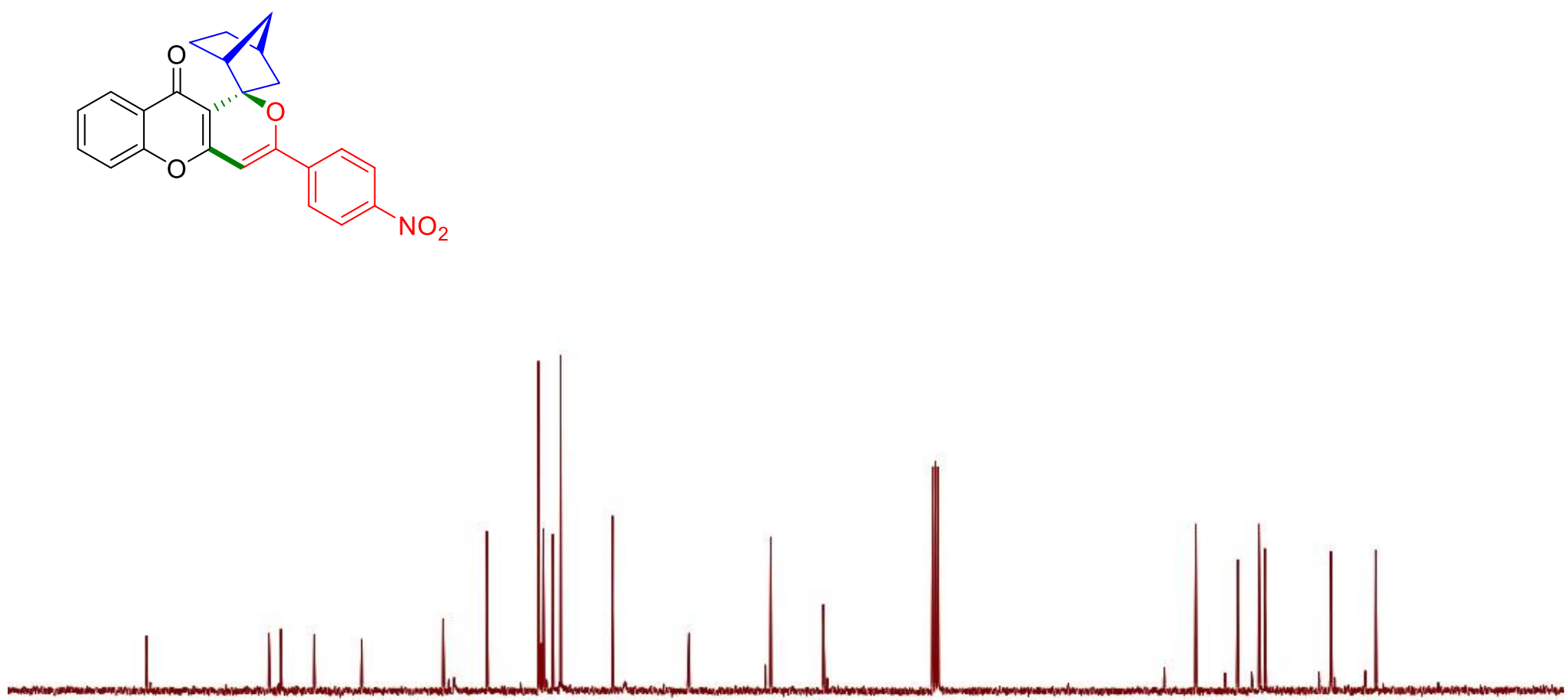

190
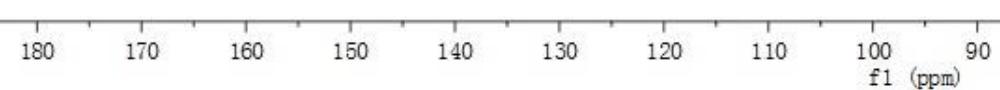
Scheme 2, 4z
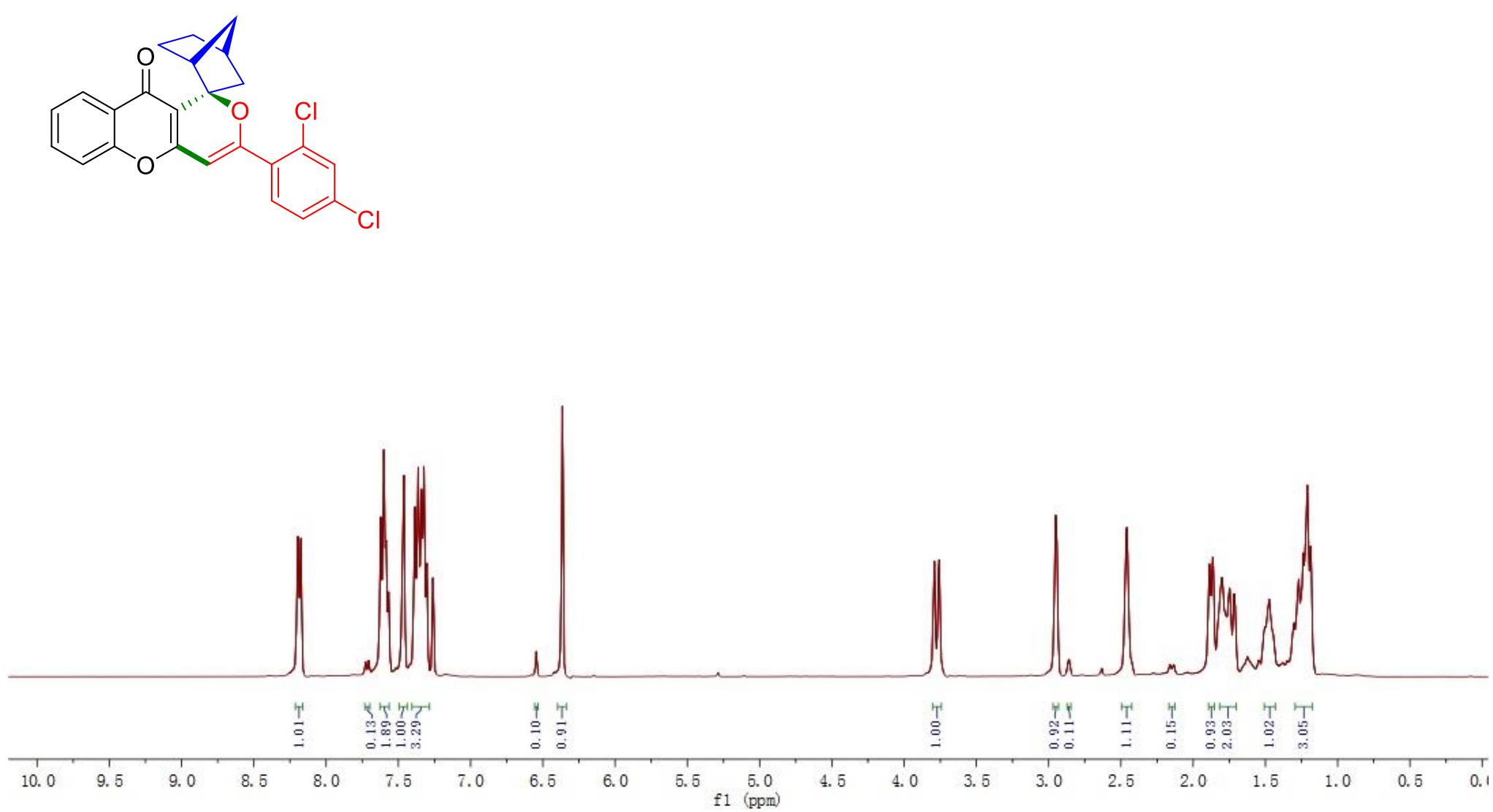


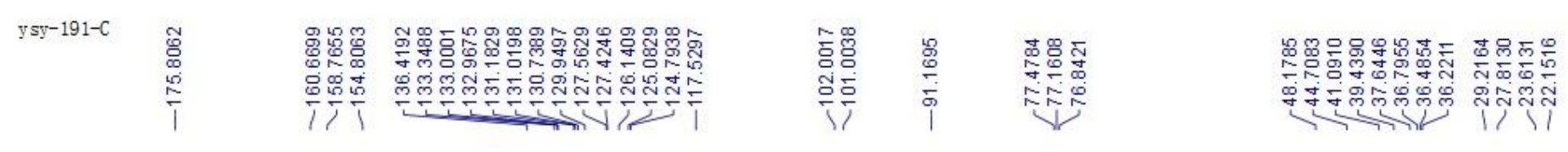

Scheme 2, 4z
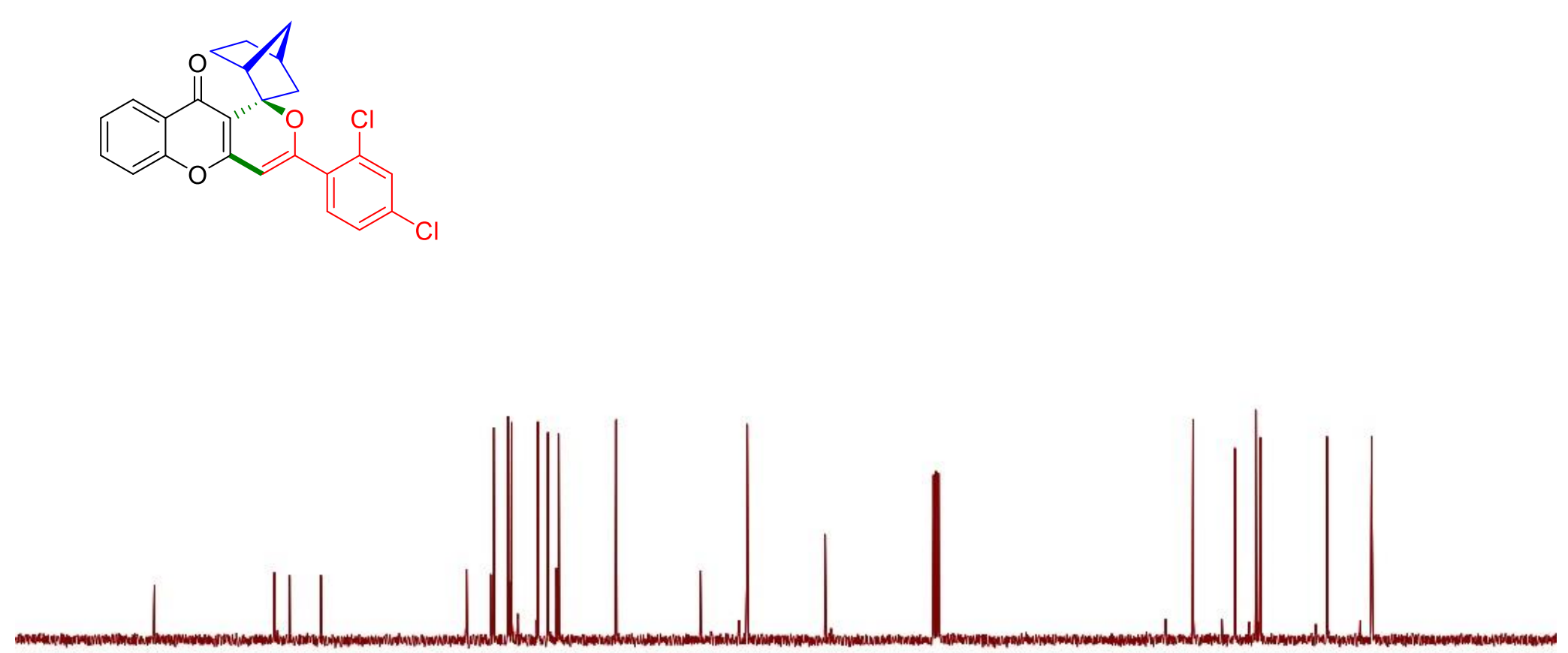


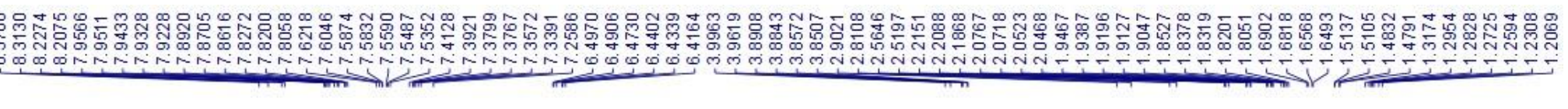

$$
\begin{aligned}
& 28
\end{aligned}
$$

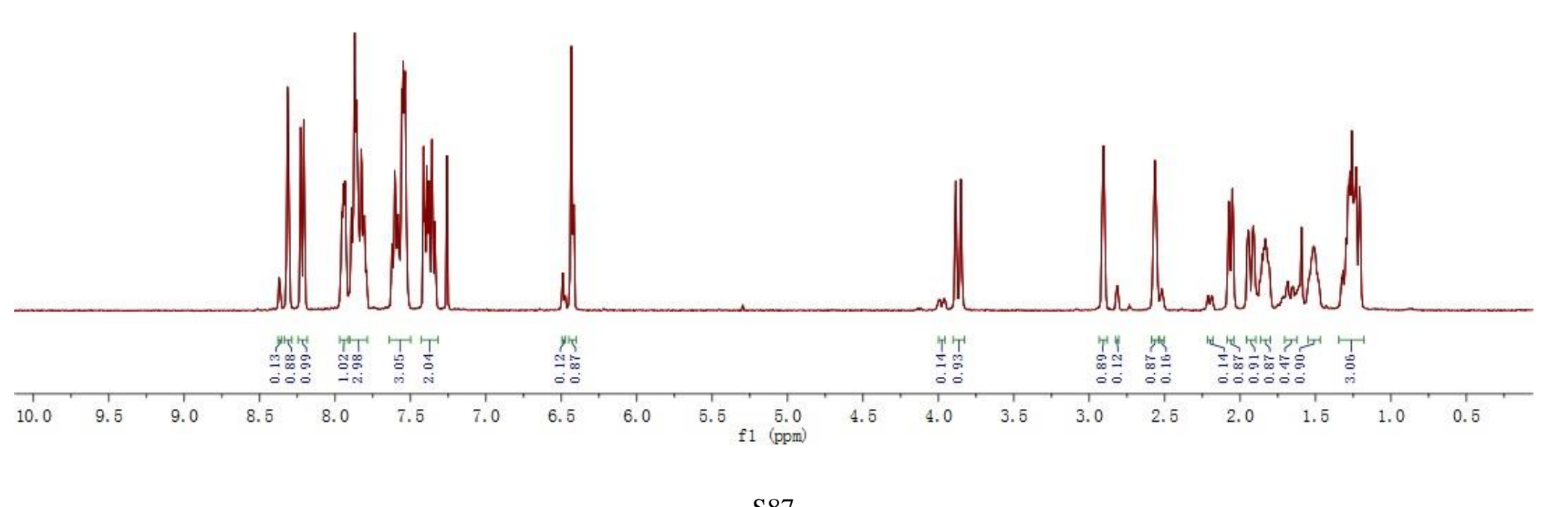


Scheme 2, 4aa
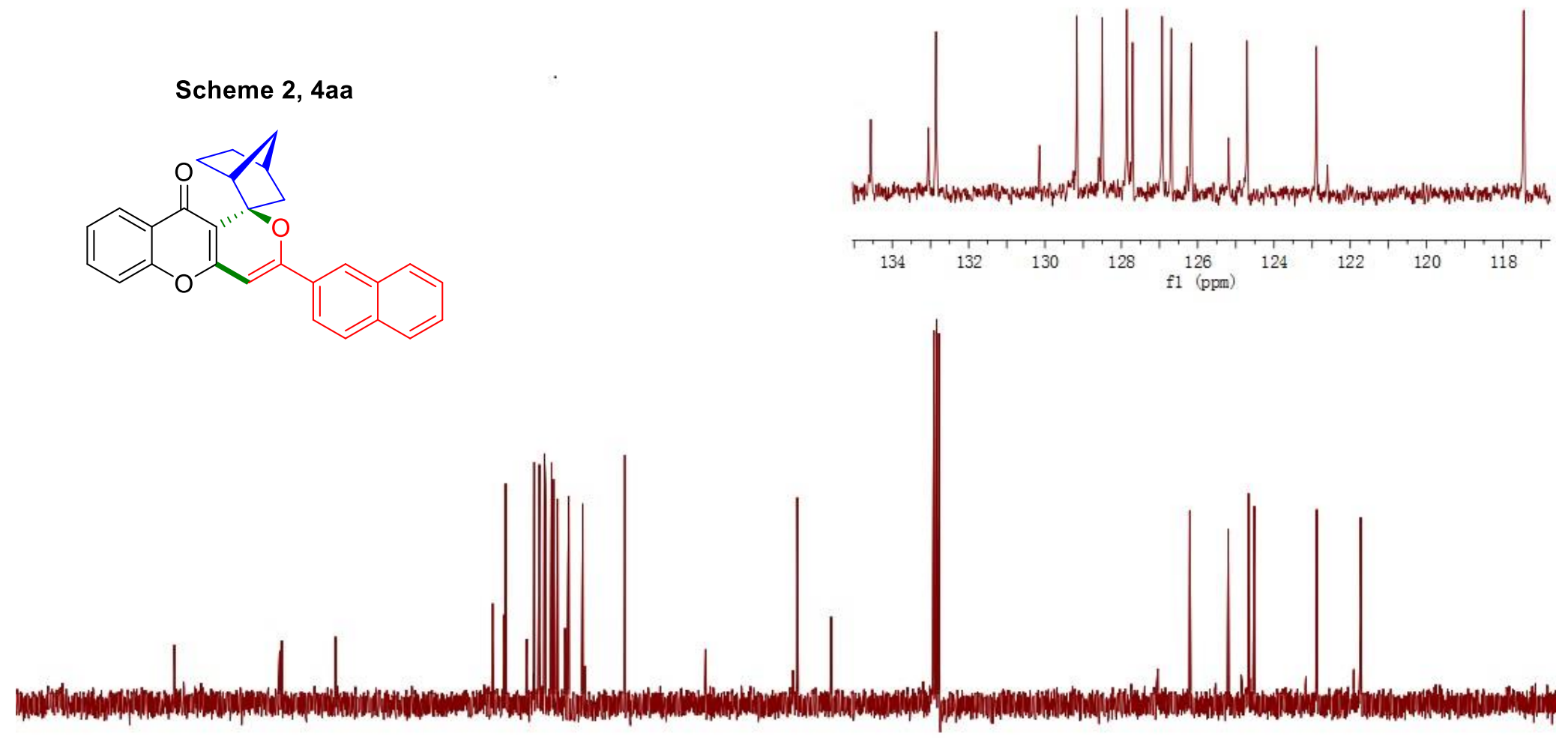
Scheme 2, 4ab
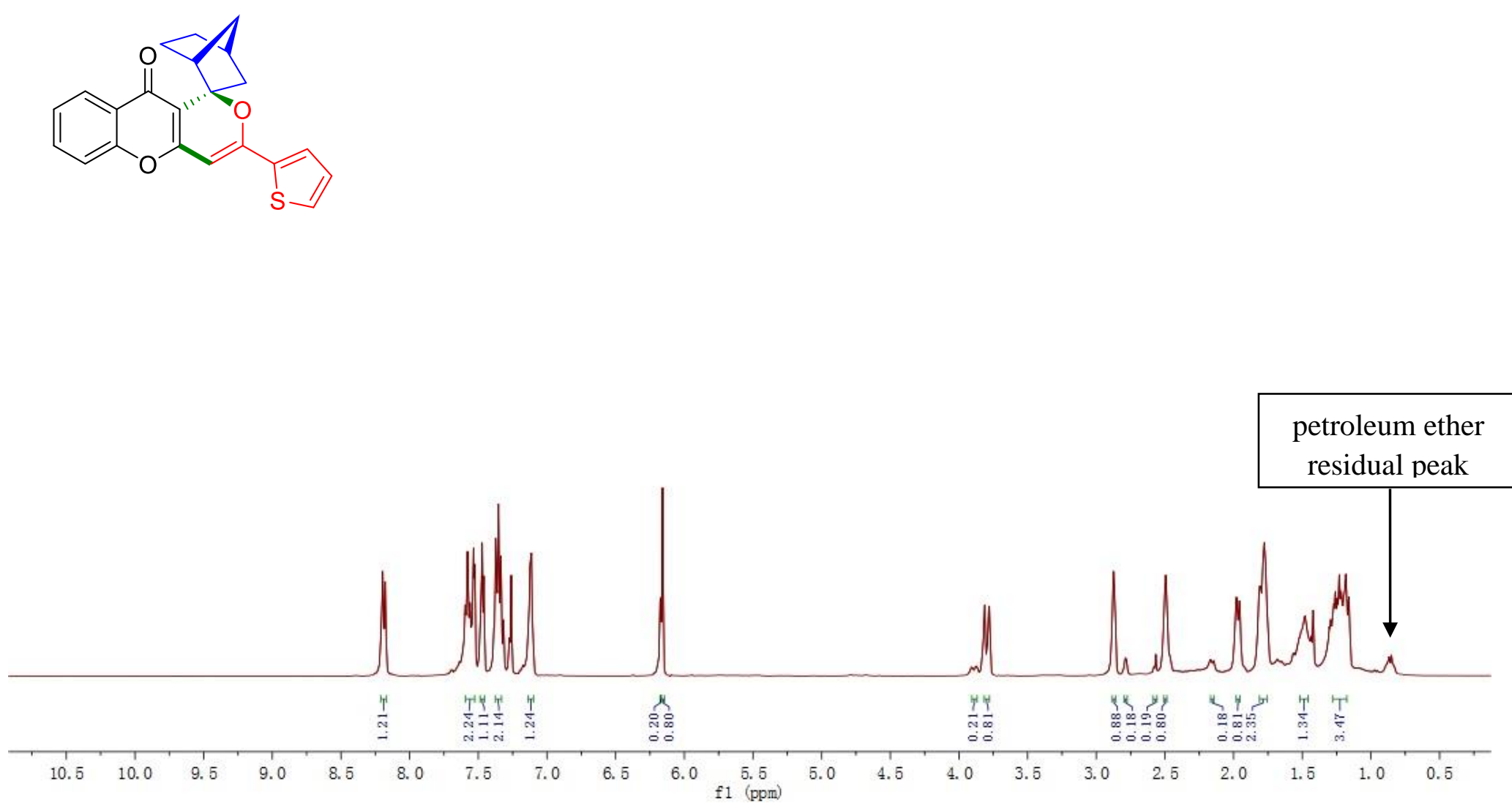


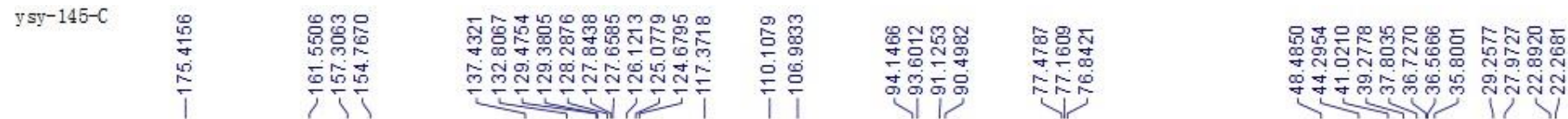

Scheme 2, 4ab<smiles>O=c1c2c(oc3ccccc13)C=C(c1cccs1)OC21C2CC3CC(C2)CC1C3</smiles>

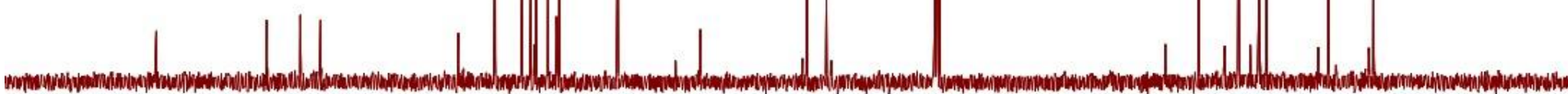


Scheme 2, 4ac
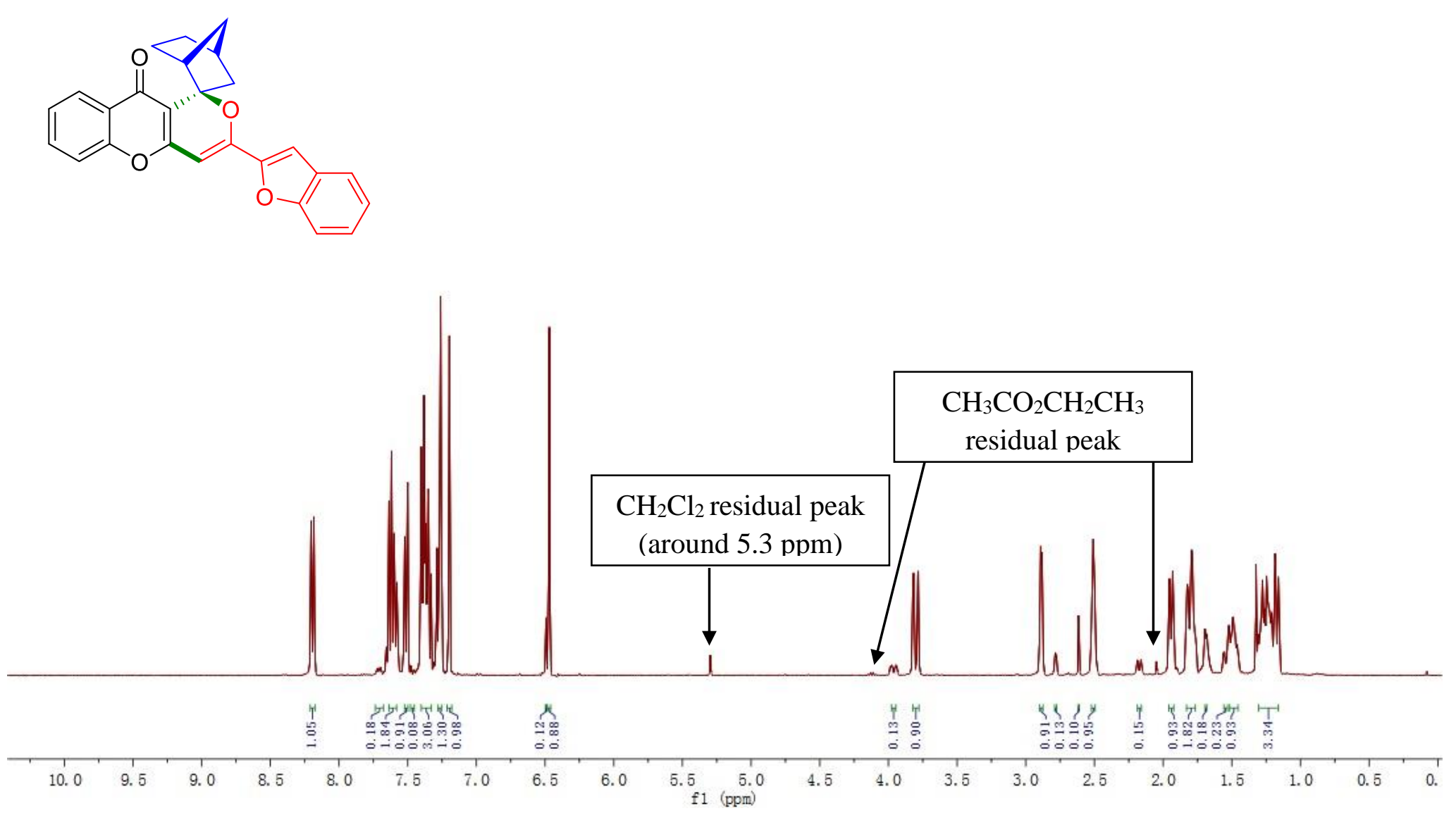
Scheme 2, 4ac
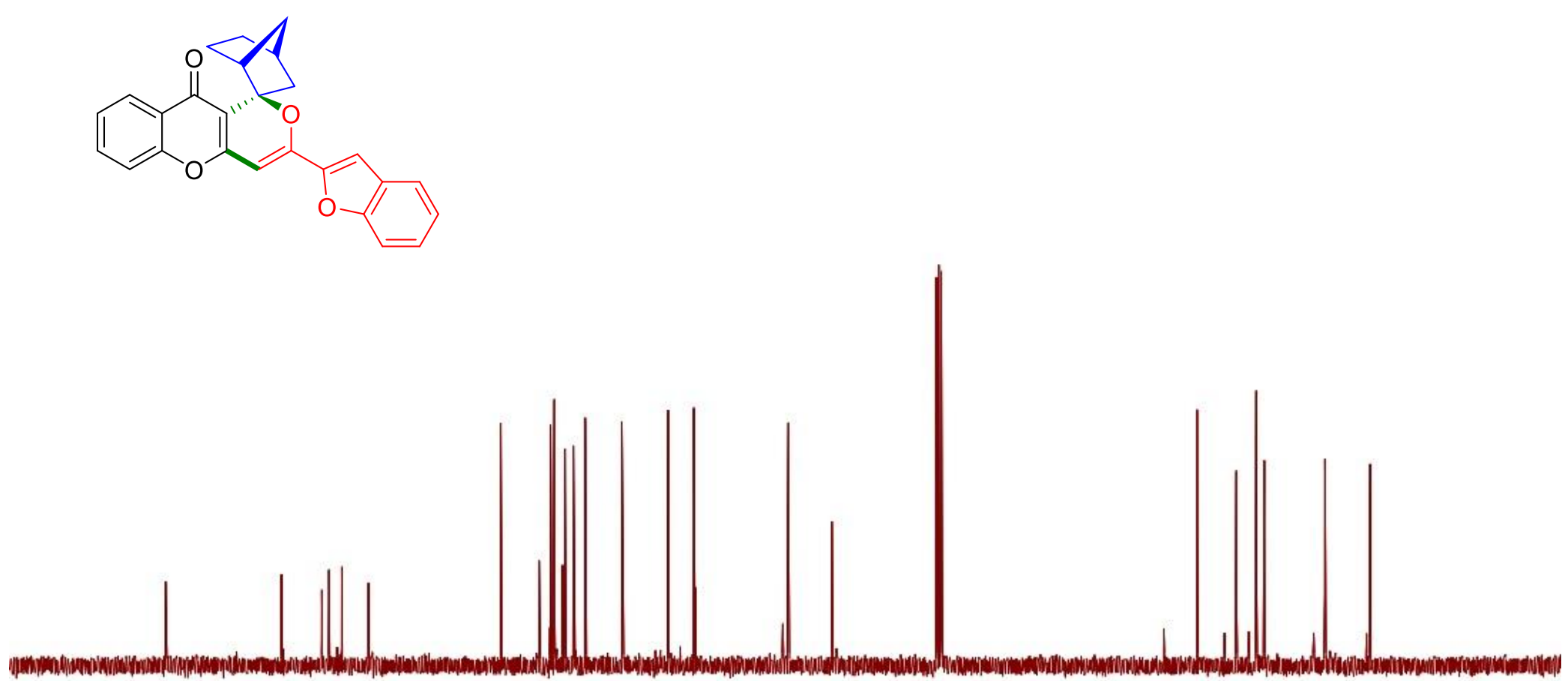

190

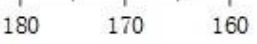

150
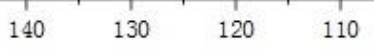

100 f1 (ppm) 
Scheme 2, 4ad<smiles>O=c1c2c(oc3ccccc13)C=C(c1cccnc1)O[C@]21CC2CC[C@@H]1C2</smiles>

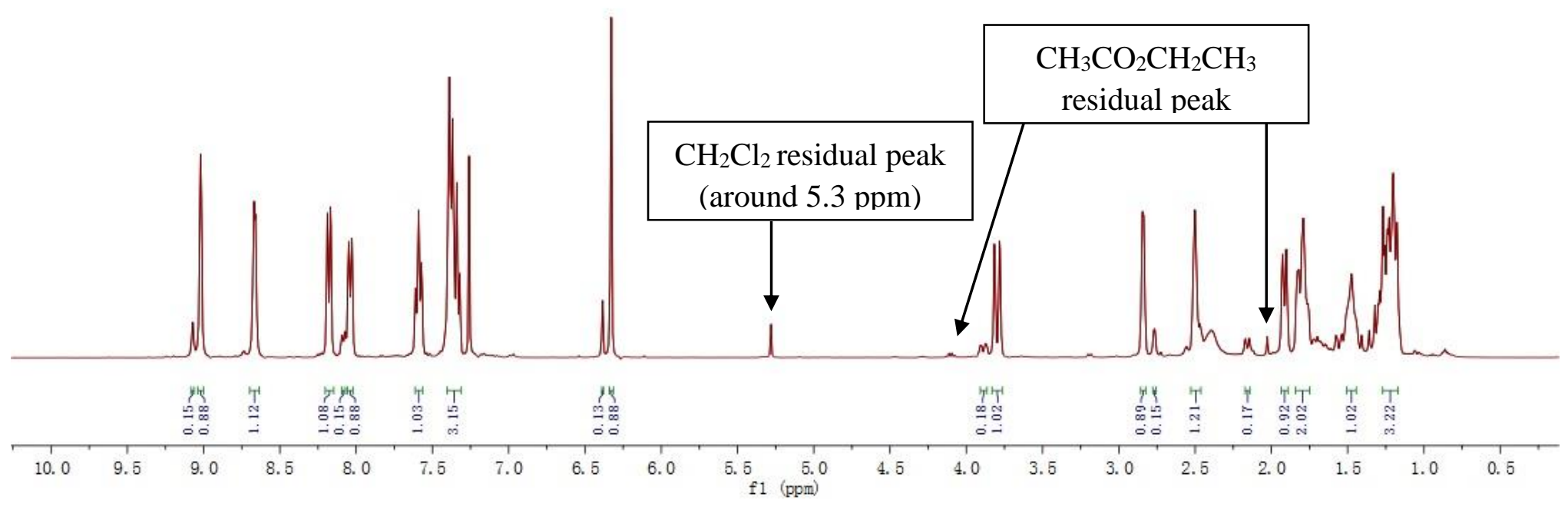


ysy-192-C

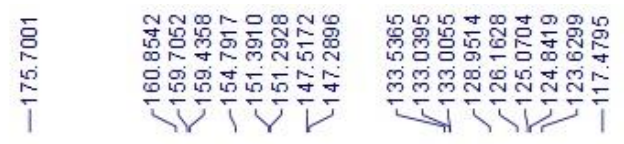

m
ले
$\frac{1}{1}$

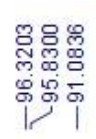

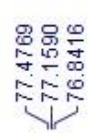

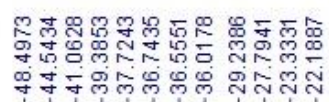

Scheme 2, 4ad<smiles>O=c1c2c(oc3ccccc13)C=C(c1cccnc1)O[C@]21C[C@H]2CC[C@@H]1C2</smiles>
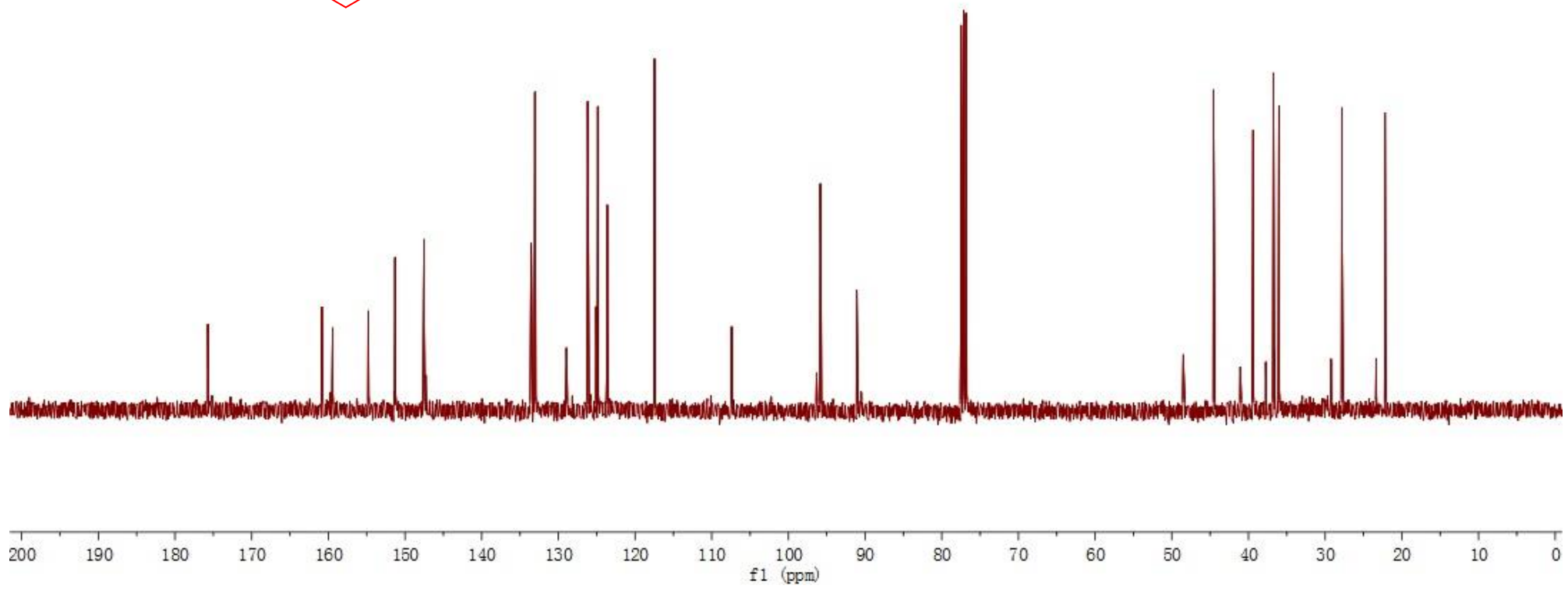

S94 


\section{Scheme 2, 4ae}
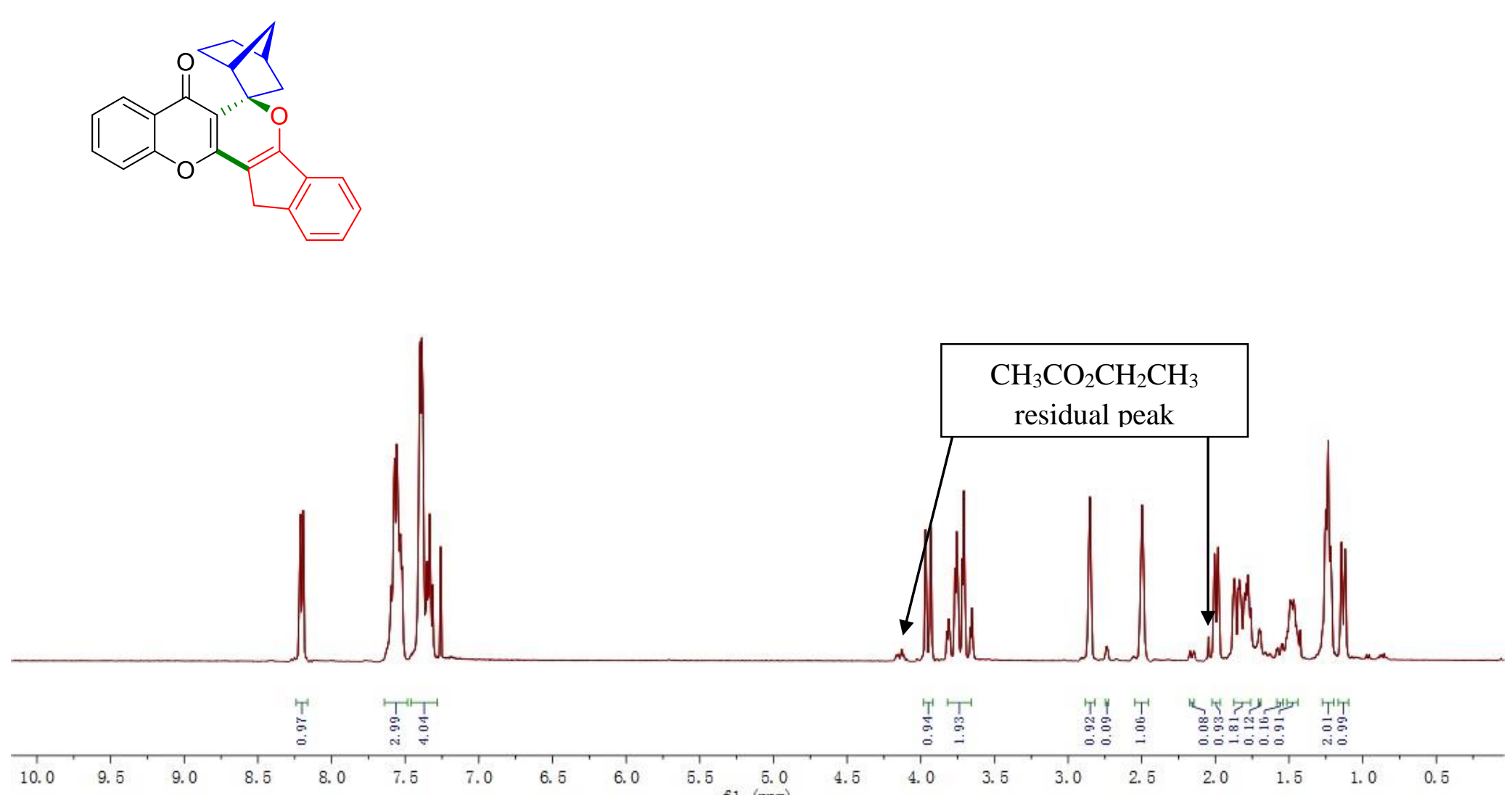


\section{ysy- $091-C$

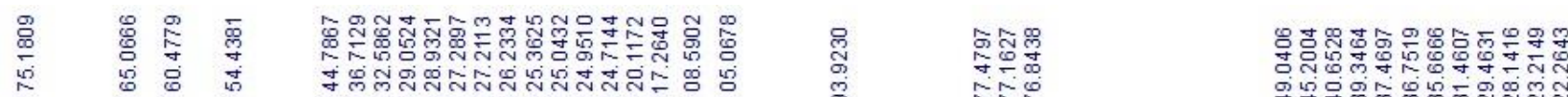

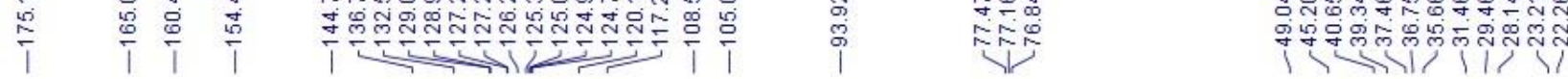

Scheme 2, 4ae<smiles>O=c1c2ccccc2oc2c3c(oc12)-c1ccccc1C3[C@H]1C[C@H]2CC[C@@H]1C2</smiles>
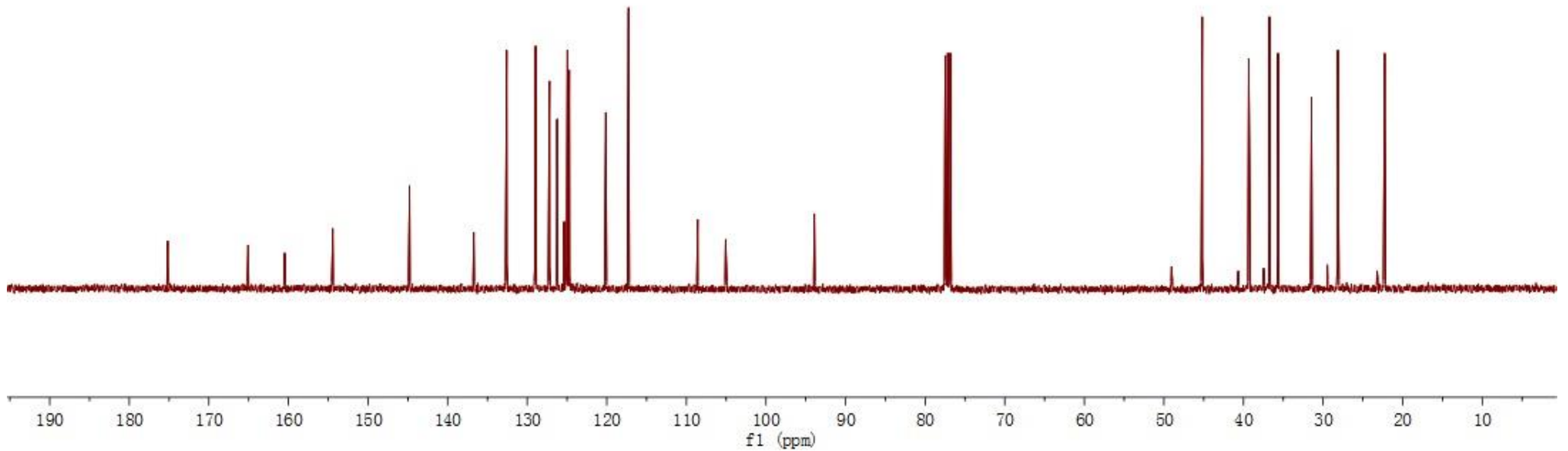


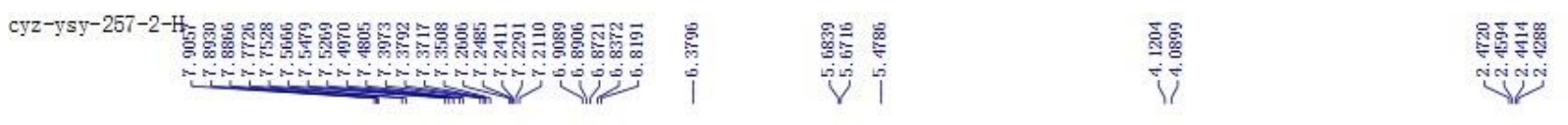

Figure 1, 5
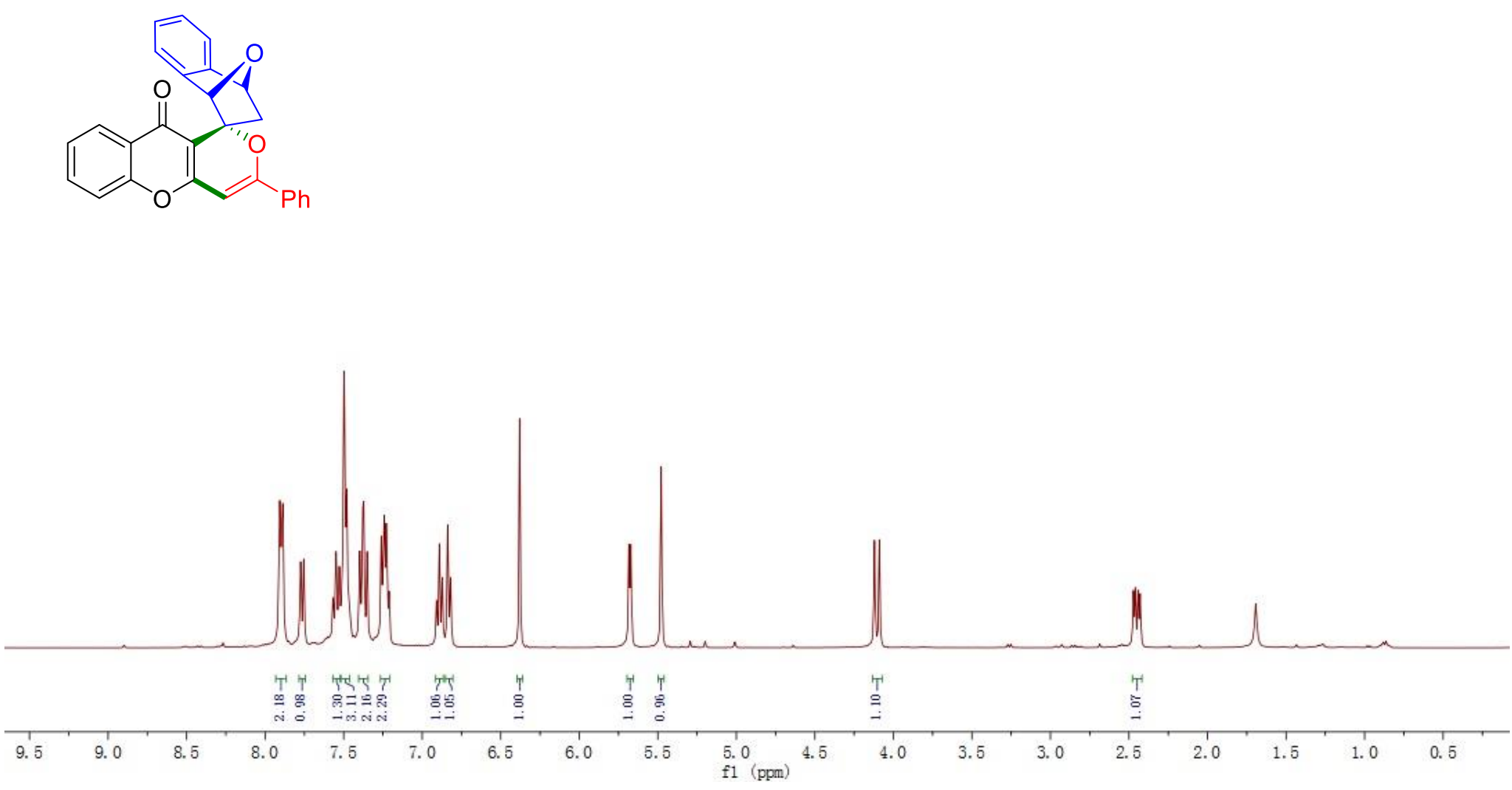
Figure 1, 5
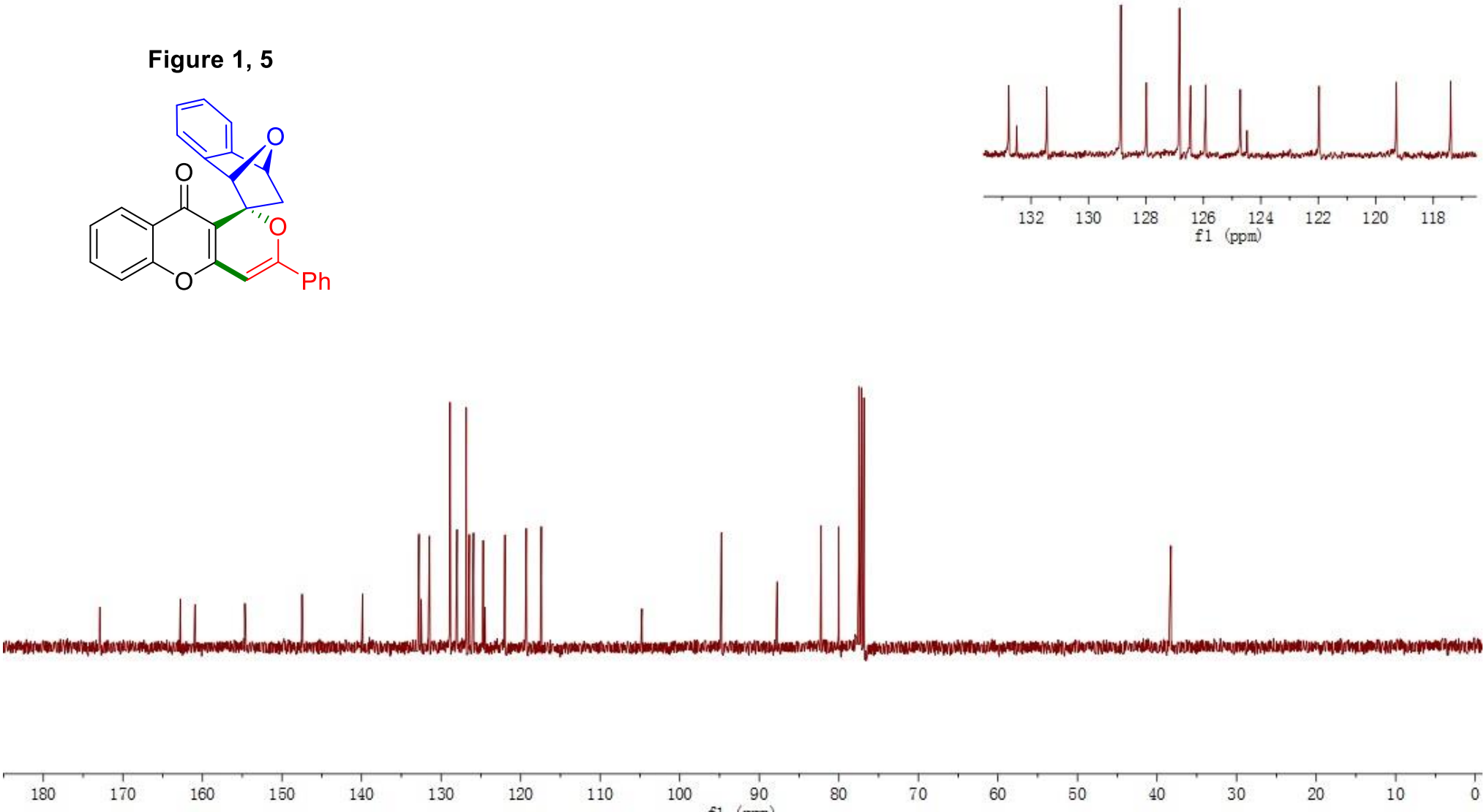

120

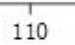

100
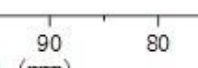

70

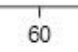

50

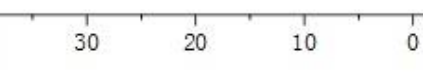


<smiles></smiles>
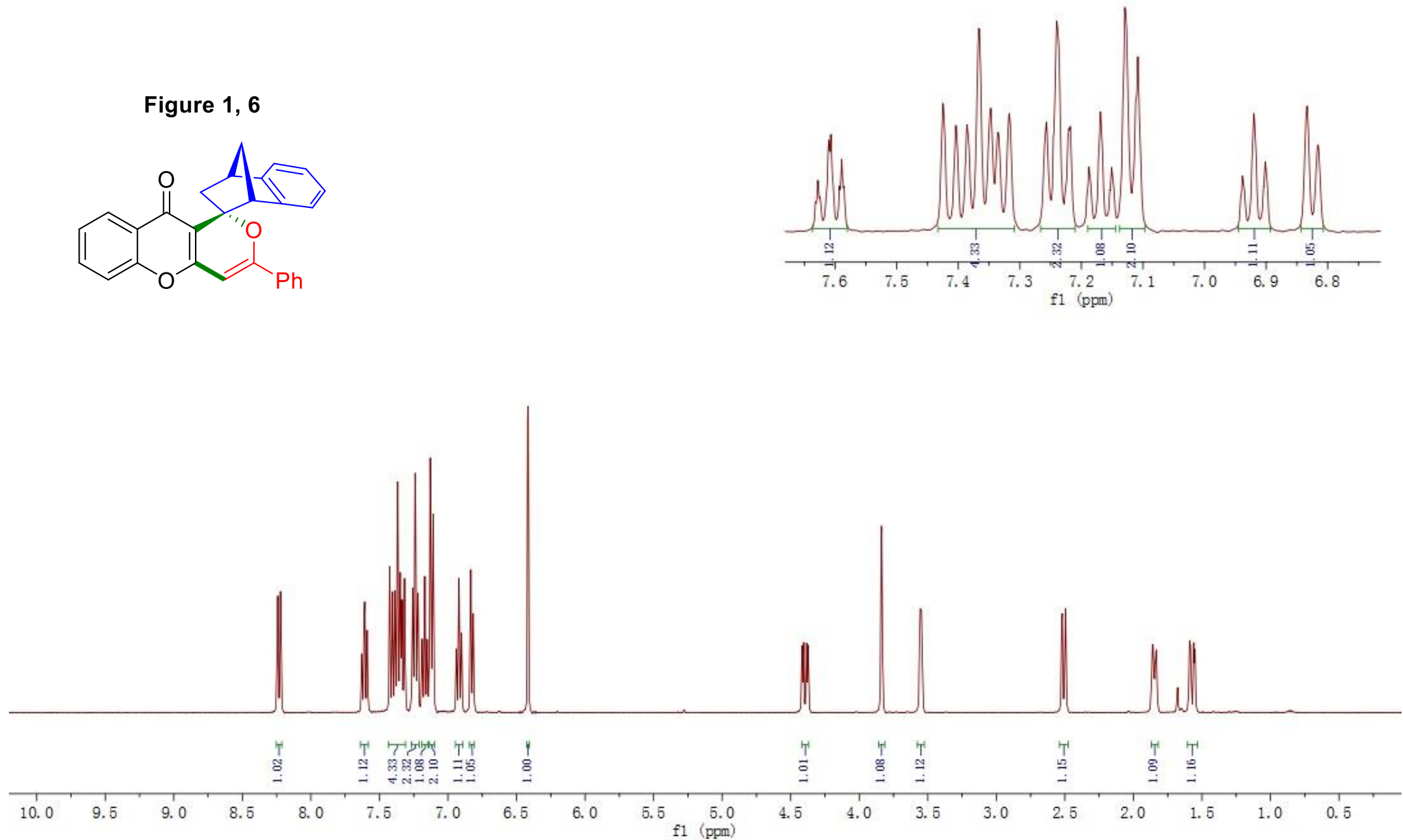
Figure 1, 6
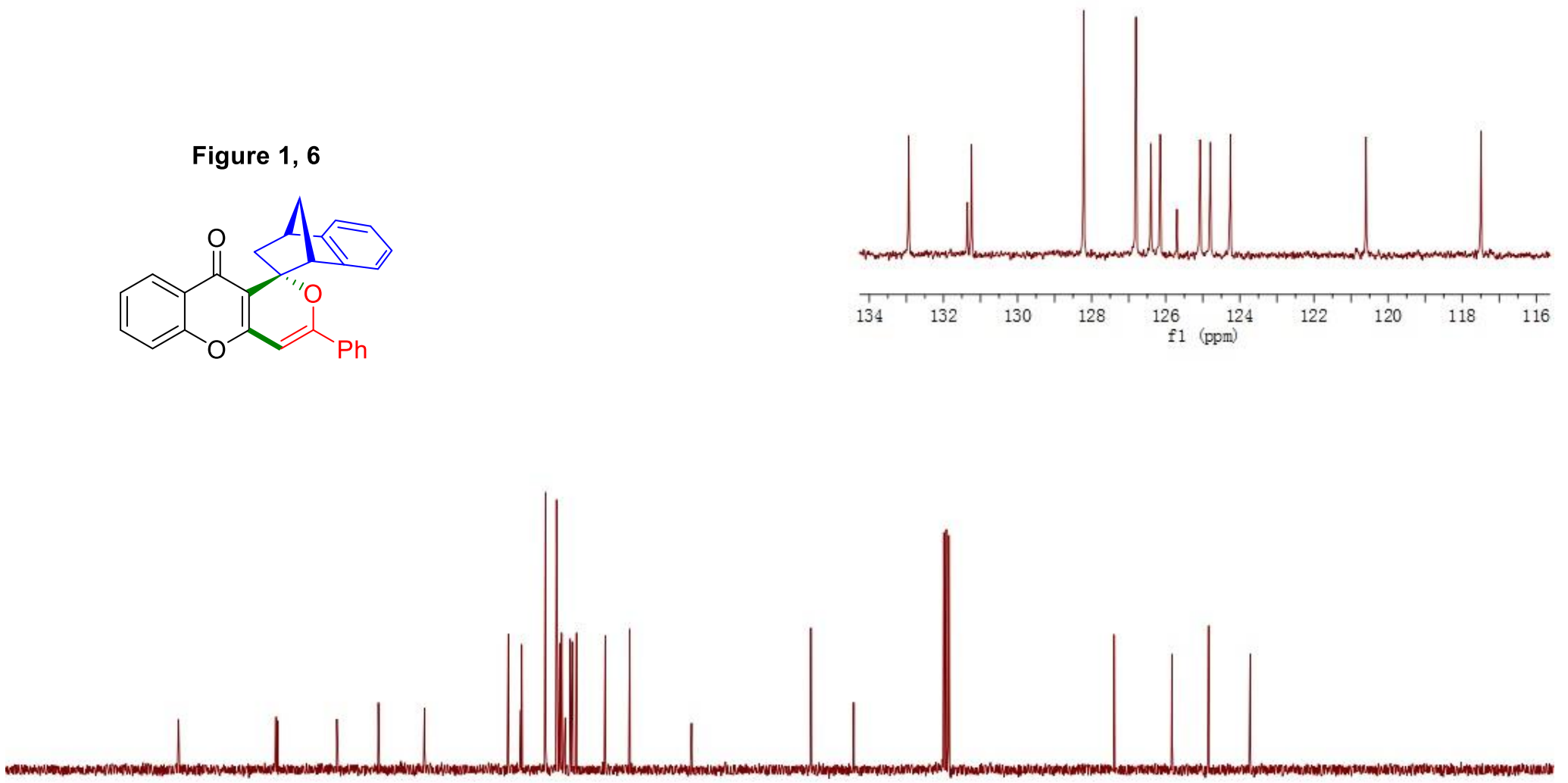
Figure 1, 7
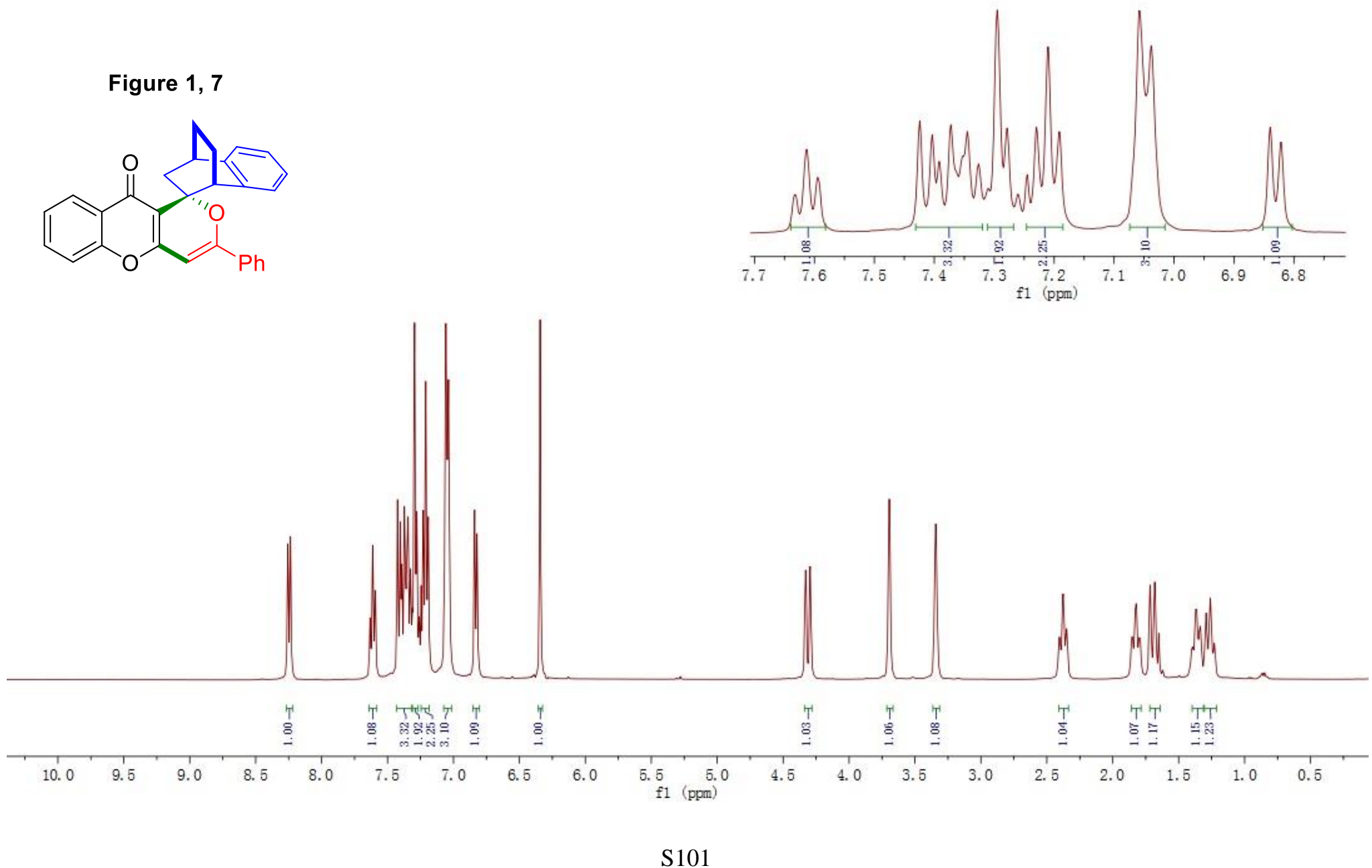

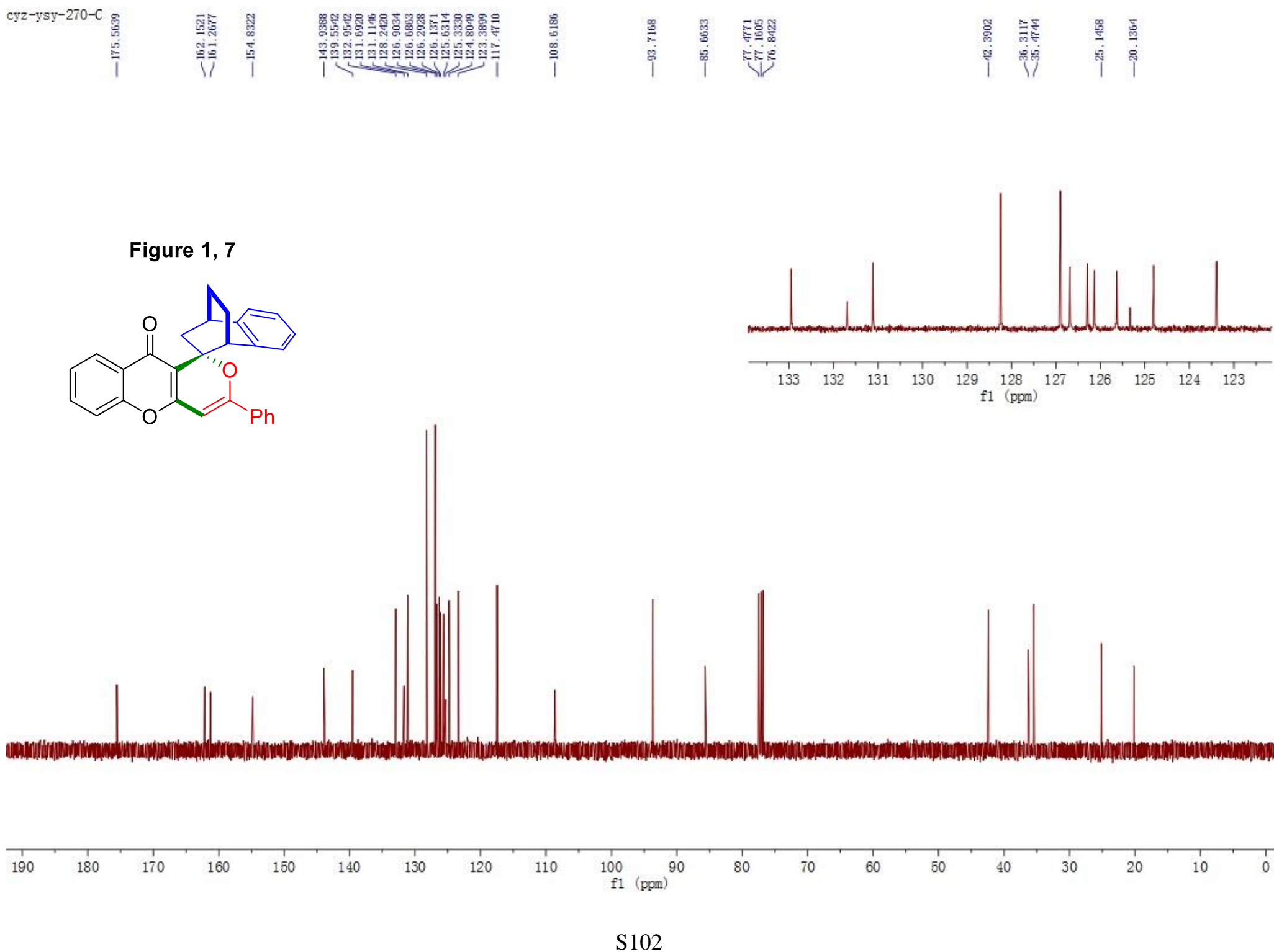
Scheme 3a, 8
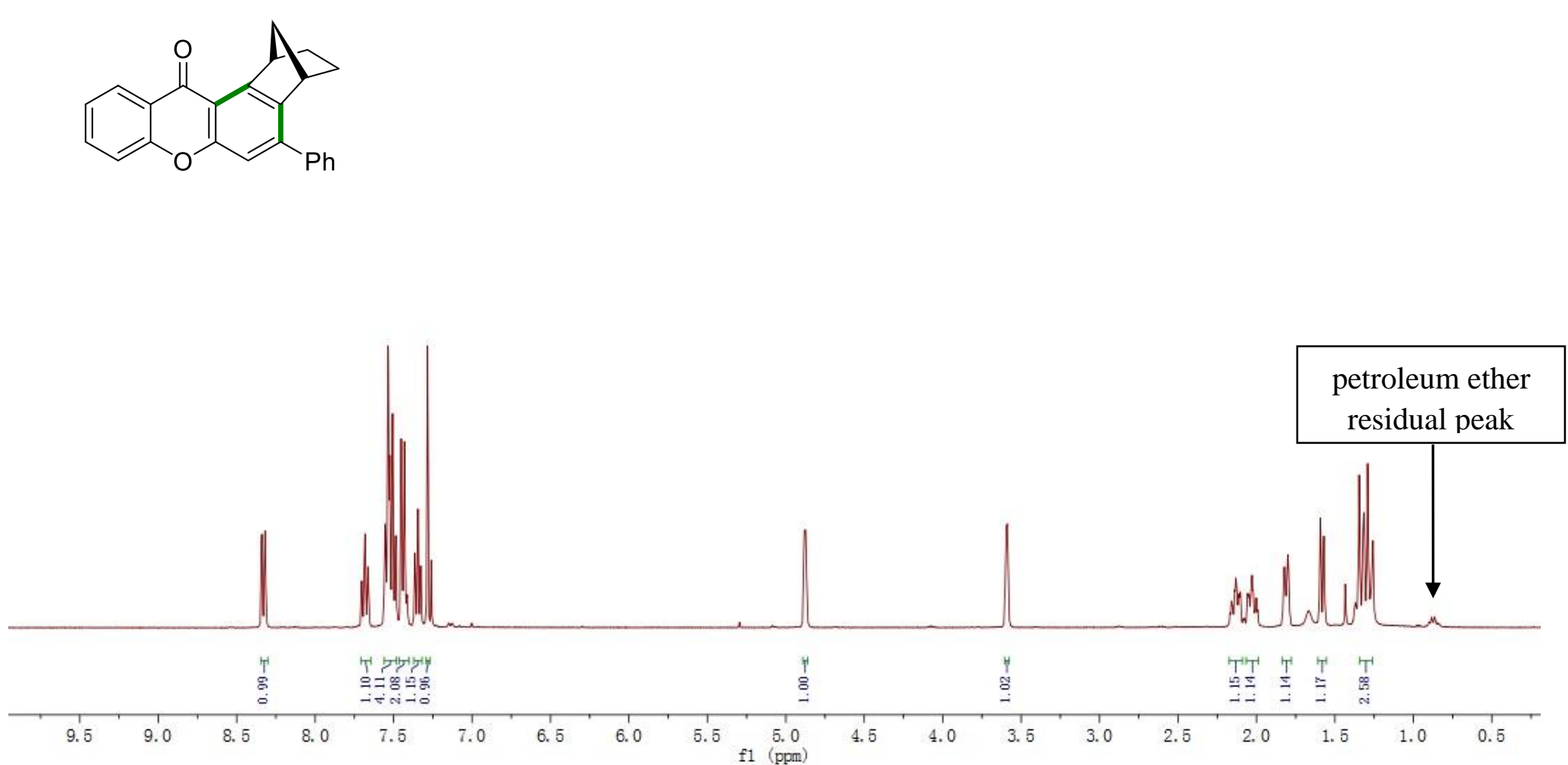


\begin{tabular}{|c|c|c|c|}
\hline $\begin{array}{l}\text { ysy-249-c } \\
\text { y1 }\end{array}$ & 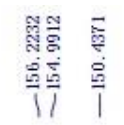 & 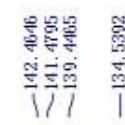 & 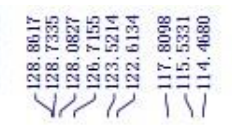 \\
\hline
\end{tabular}

Scheme 3a, 8
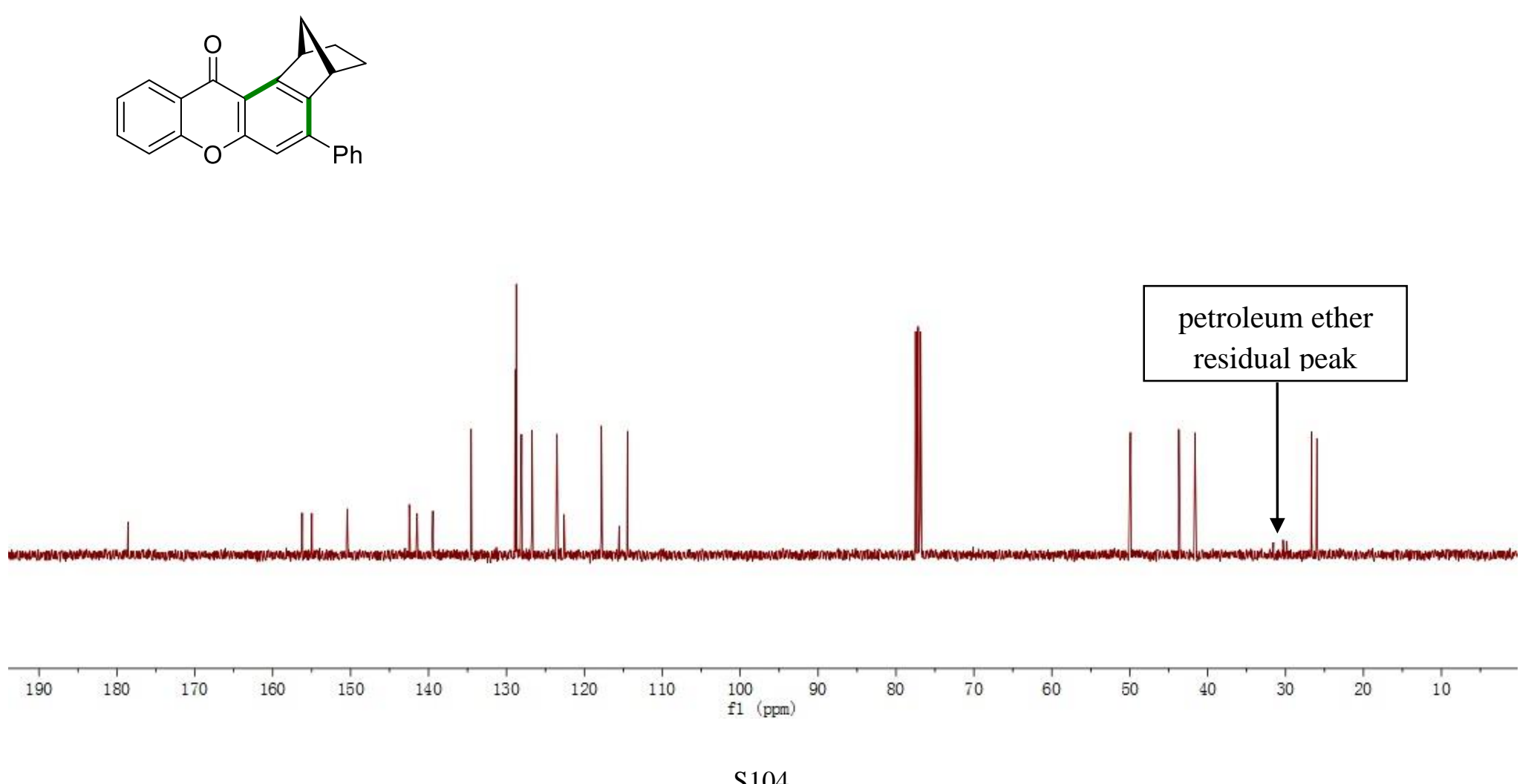
ysy-253-H

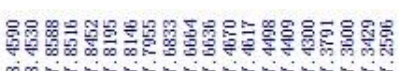

$405-2+25+2$

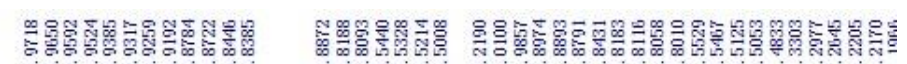

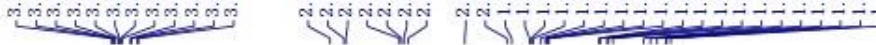

Scheme 3b, 9
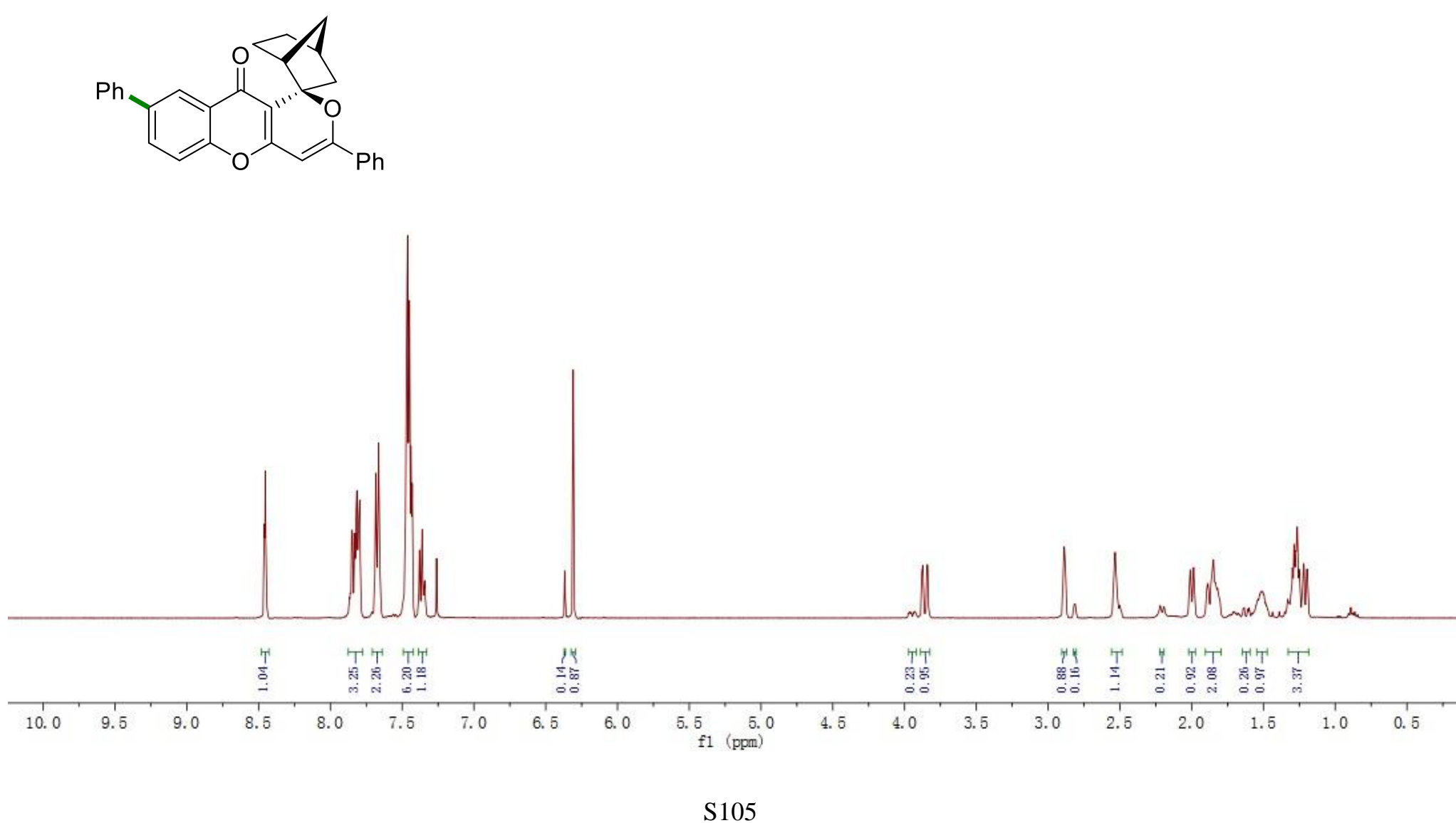
ysy-253-c
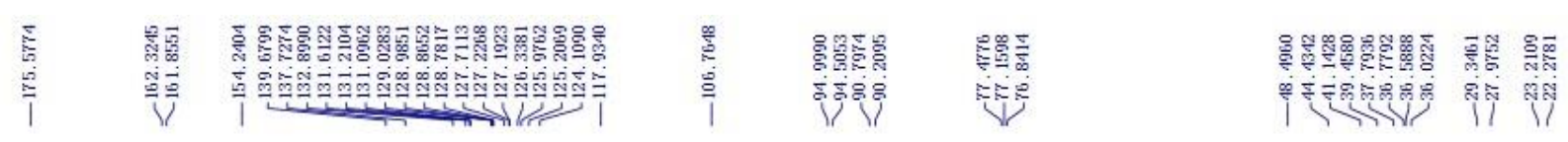

Scheme 3b, 9
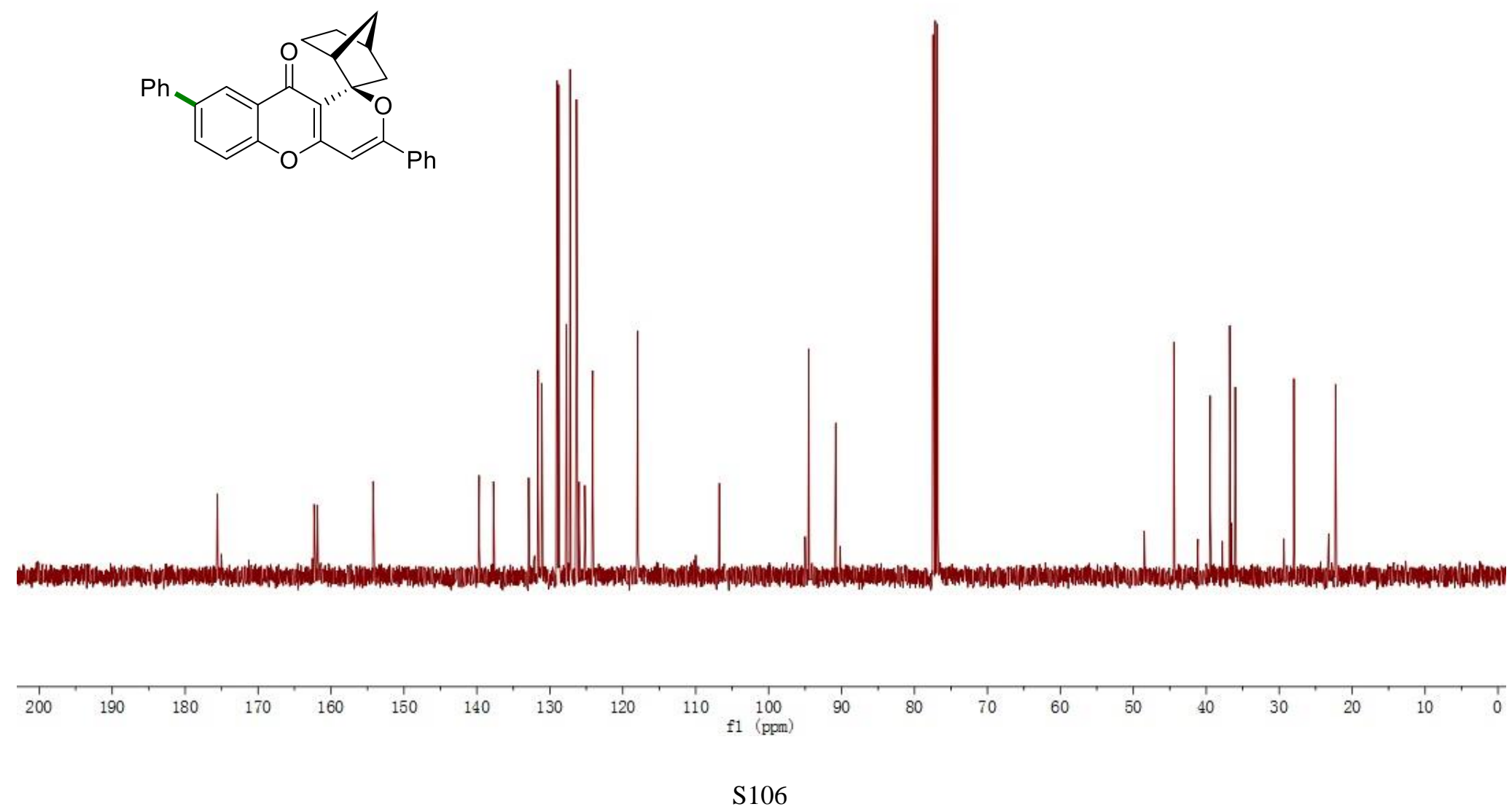
Scheme 4a, 4a'<smiles>O=c1c2c(oc3ccccc13)C1C3CCC(C3)C21</smiles>

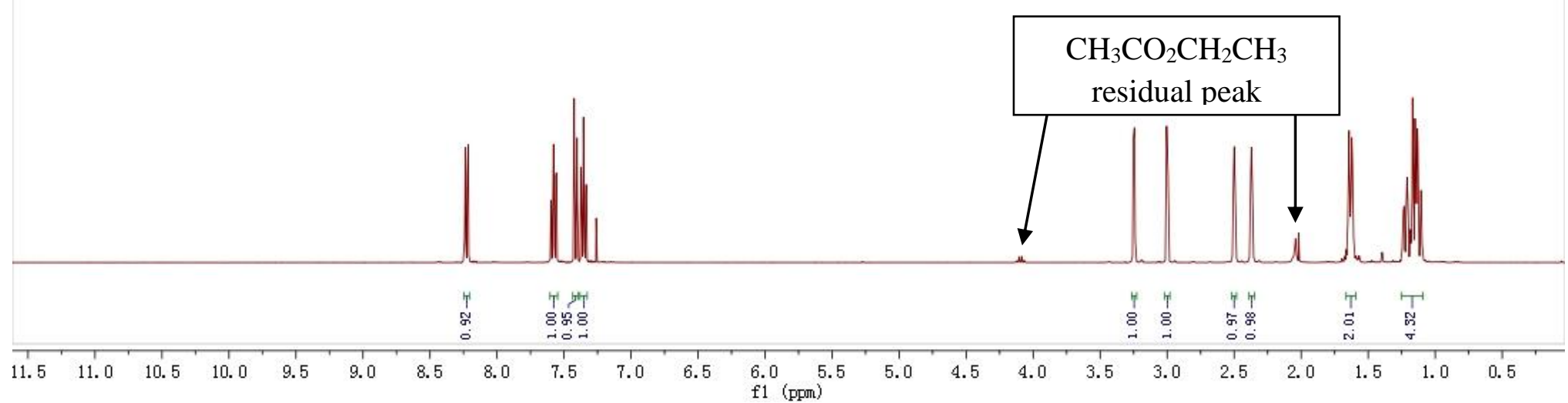


cyz-hc-286-C

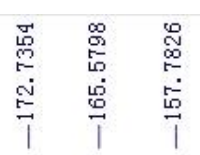

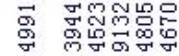

กั่

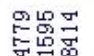

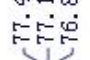

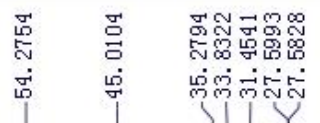

Scheme $4 a, 4 a^{\prime}$
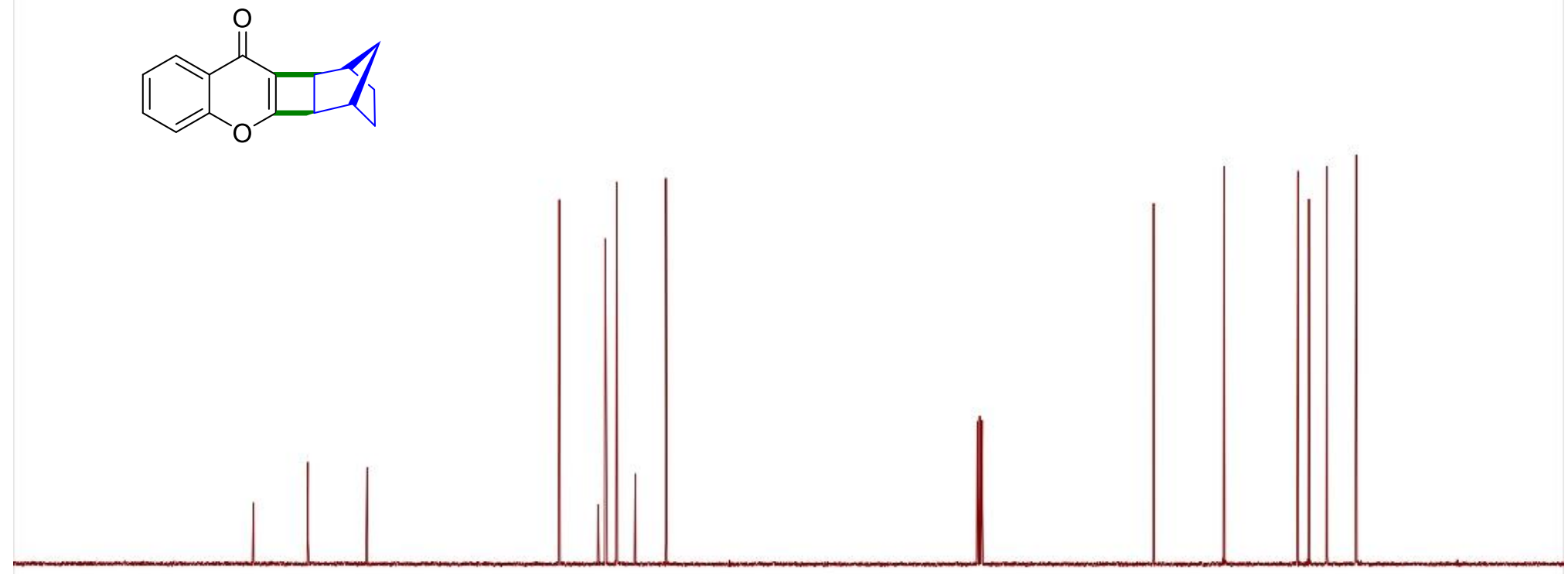

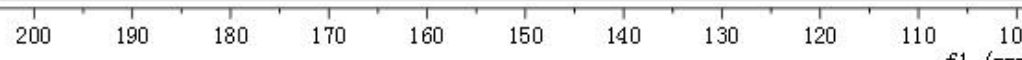
f1 $\begin{array}{r}100 \\ (\mathrm{pppm})\end{array}$ 
12. ${ }^{1} \mathrm{H}$ NMR and HRMS spectra of $4 \mathrm{a}-D$.

4a-D
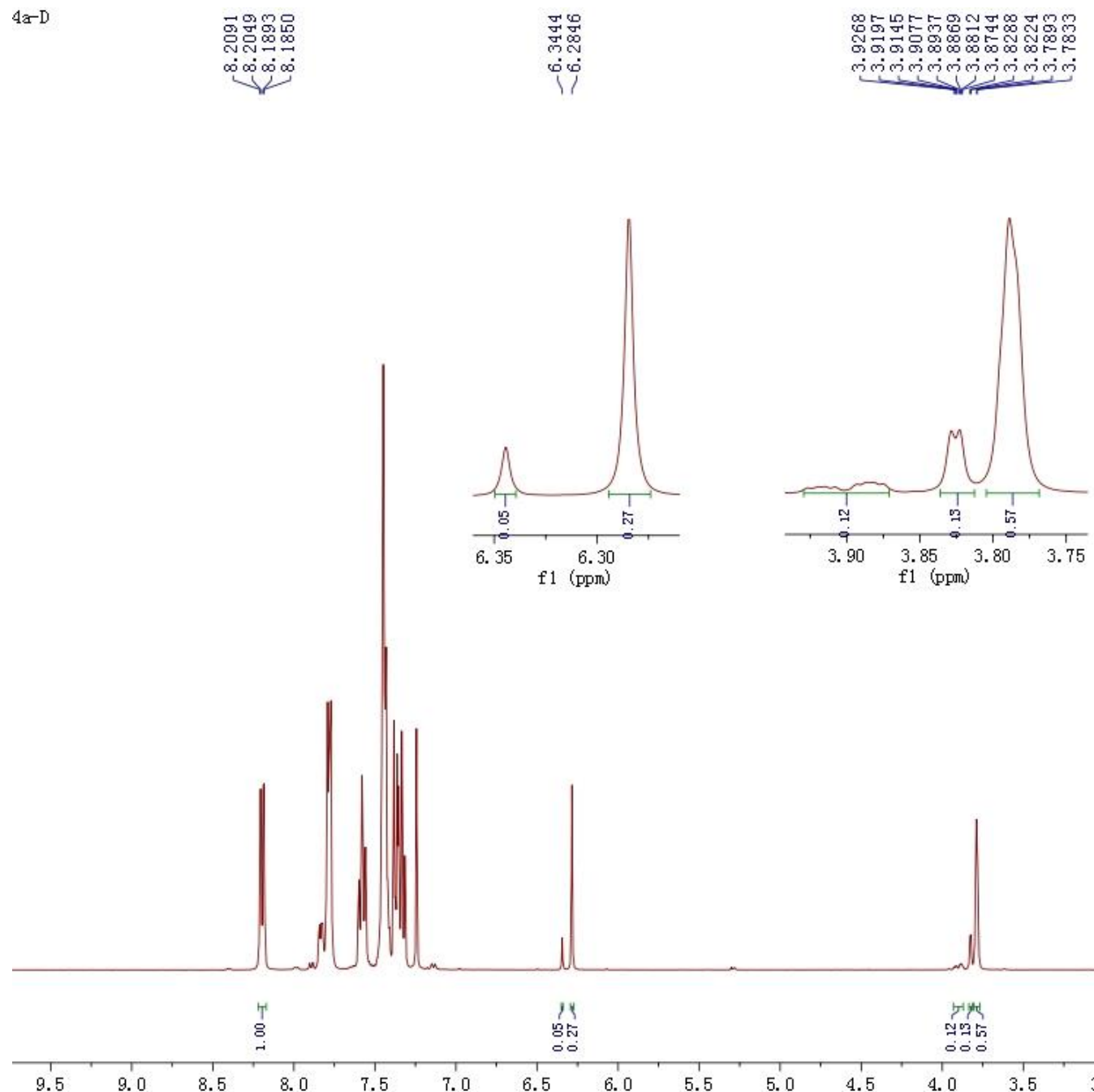

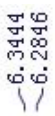

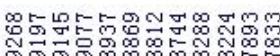

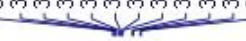

Scheme 4b, 4a-D

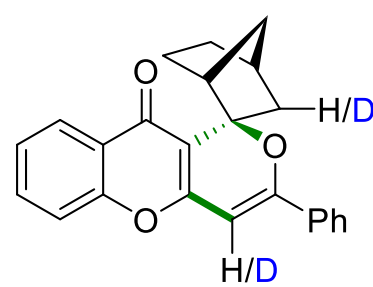



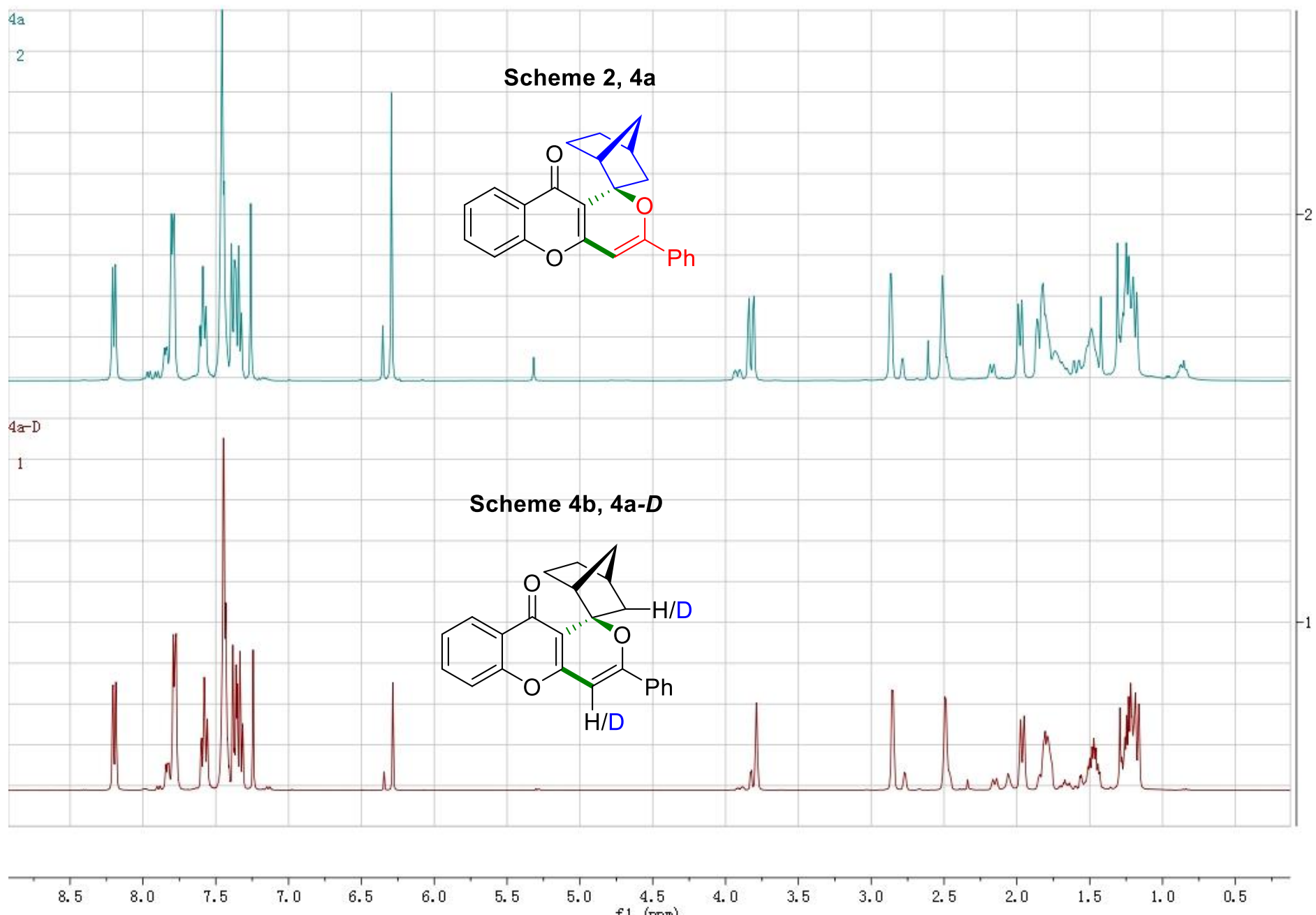


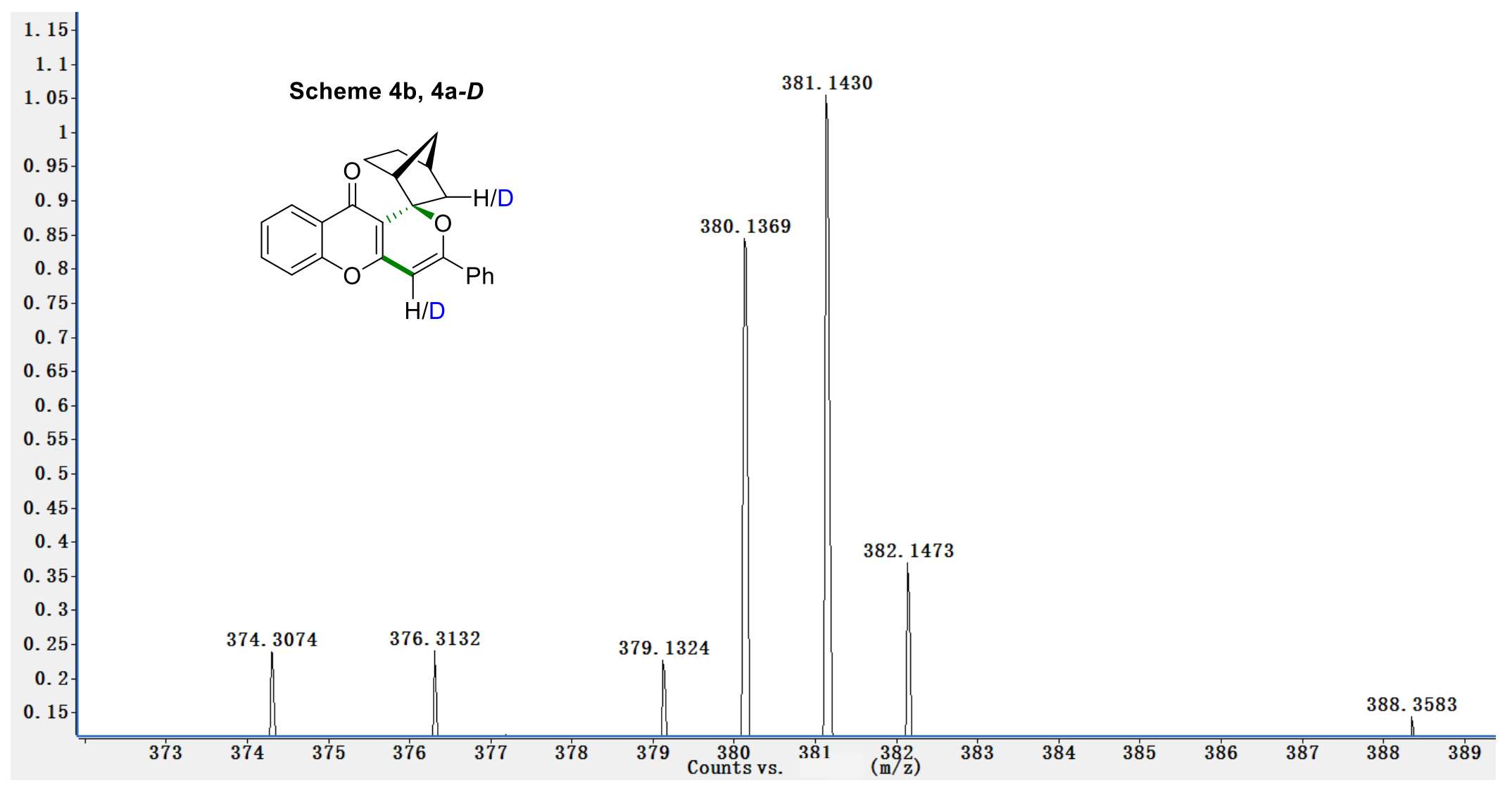

HRMS (ESI-TOF): calcd. for $\mathrm{C}_{24} \mathrm{H}_{20} \mathrm{NaO}_{3}[\mathrm{M}+\mathrm{Na}]^{+} 379.1305$; found 379.1324 .

HRMS (ESI-TOF): calcd. for $\mathrm{C}_{24} \mathrm{H}_{19} \mathrm{DNaO}_{3}[\mathrm{M}+\mathrm{Na}]^{+} 380.1367$; found 380.1369 .

HRMS (ESI-TOF): calcd. for $\mathrm{C}_{24} \mathrm{H}_{18} \mathrm{D}_{2} \mathrm{NaO}_{3}[\mathrm{M}+\mathrm{Na}]^{+} 381.1430$; found 381.1430 . 